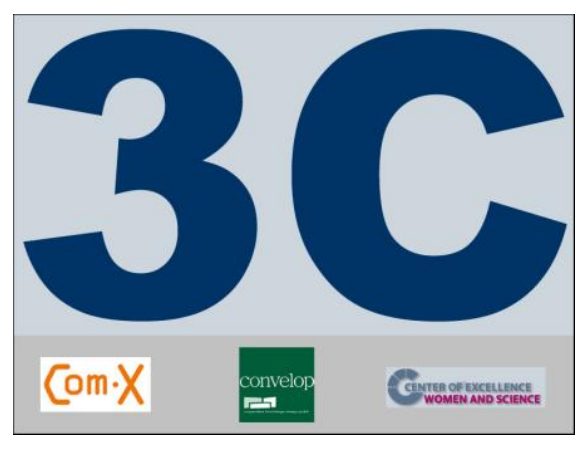

\title{
Evaluierung des Programms FEMtech
}

Endbericht | Durchführungszeitraum: August 2010 bis Jänner 2011

AuftraggeberIn: Bundesministerium für Verkehr, Innovation und Technologie

\section{Projektnummer:}

AutorInnen:
1000_FEMtech-Eval_1002-1

Bearbeitung durch Karin Grasenick, Stephan Kupsa, Nicole Warthun

Basierend auf dem Bericht von:

Gabriele Gerhardter, Nicole Warthun, Andrea Löther, Markus Gabriel, Nina Steinweg, Stephan Kupsa, Erich Domke, Martina Süssmayer

02. Mai 2011 convelop

cooperative knowledge design gmbh

A-8010 Graz Bürgergasse 8-10/l Telefon: $\quad+43(0) 316720813$

Fax: $\quad+43(0) 316720813-20$ www.convelop.at office@convelop.at
Com.X Institut für Kommunikationsanalyse und Evaluation

D-44789 Bochum Ehrenfeldstr. 34

Tel.: $\quad$ +49 (0) 2343250830

Fax: $\quad$ +49 (0) 2343250831

www.comx-forschung.de
GESIS Leibniz-Institut für

Sozialwissenschaften / CEWS

D-68159 Mannheim Quadrat B2, 1

Tel.: $\quad+49(0) 2282281522$

Fax: $\quad+49(0) 2282281550$

www.gesis.org www.cews.org 


\section{Kurzfassung}

\section{Das Programm FEMtech}

FEMtech ist ein 2004 initiiertes Programm des Bundesministeriums für Verkehr, Innovation und Technologie (BMVIT) zur Förderung von Frauen und zur Schaffung von Chancengleichheit in Forschung und Technologie. Das Programm wird im Rahmen von fFORTE (Frauen in Forschung und Technologie) durchgeführt, einer auf Empfehlung des Rats für Forschung und Technologieentwicklung 2002 ins Leben gerufenen ministerienübergreifenden Dachinitiative. FEMtech zielt darauf ab, Rahmenbedingungen und Karrierechancen für bereits berufstätige Frauen und (potenzielle) Berufseinsteigerinnen in Unternehmen und Organisationen der industriellen und außeruniversitären Forschung zu verbessern. Dadurch soll die Attraktivität technischer und naturwissenschaftlicher Berufe für Frauen gesteigert werden, um insgesamt die Anzahl weiblicher Beschäftigter zu erhöhen. Seit Mitte 2008 bis Ende 2010 liegt die Durchführung des Programms beim FEMtech Kompetenzzentrum in Kooperation mit der Österreichischen Forschungsförderungsgesellschaft (FFG). Das Kompetenzzentrum ist vertreten durch die Österreichische Gesellschaft für Umwelt und Technik (ÖGUT), Joanneum Research Forschungsgesellschaft, Bohmann Druck und Verlag sowie D\&Z Consulting.

\section{Die Evaluation}

Mit der Durchführung der FEMtech Programmevaluation im Zeitraum August 2010 bis Anfang Februar 2011 hat das BMVIT die ARGE "3C" beauftragt, bestehend aus der Forschungs- und Beratungseinrichtung convelop (Graz, Wien) sowie den deutschen Partnerinstituten com.X (Bochum) und CEWS, ein Bereich des Leibniz-Instituts GESIS (Bonn).

Ziel der Evaluierung war die Bewertung der mittelfristigen Effekte und Wirkungsrichtungen (bei einer Laufzeit von ca. 7 Jahren), um einen Beitrag für die Programmweiterentwicklung zu leisten. Dabei kam ein pragmatisches und fokussiertes Evaluationsdesign zum Einsatz, welches quantitative und qualitative Methoden kombiniert. Neben einer übergreifenden Dokumentenanalyse kamen zum Einsatz:

- Differenzierte Onlinebefragungen (389 FEMtech NutzerInnen und 1.583 FFGFördernehmende)

- Fokusinterviews (Leitungsebene von Unternehmen, die im Bereich Forschung, Technologie und Innovation (FTI) tätig sind, FEMtech Fördernehmende, politische AkteurInnen des Bereiches FTI (im Folgenden FTI-politische AkteurInnen genannt) sowie ehemalige Programmleiterinnen)

- Produktanalysen (FEMtech Output und internationale Fallbeispiele)

- Medienresonanz- und Nutzungsanalyse (FEMtech Website)

- Programmgespräche und Self-Assessment (FEMtech Umsetzerinnen)

\section{Zentrale Evaluationsergebnisse}

Die Basis von FEMtech bildet der Bereich „Förderungen“, er umfasst die drei Programmlinien Karriere, Karrierewege und FTI-Projekte. Gefördert werden Maßnahmen zur Verbesserung der Chancengleichheit innerhalb von Unternehmen und außeruniversitären Forschungseinrichtungen (Karriere), Kooperationen zwischen Hochschulen und Unternehmen zur Karrierevorberei- 
tung von Studentinnen in der industriellen Forschung (Karrierewege) sowie Forschungsprojekte mit Gender-Dimension (FTI-Projekte).

FEMtech besteht darüber hinaus aus einem vielfältigen Maßnahmenbündel zur Bewusstseinsschaffung und Sensibilisierung, Vernetzung und Wissensvermittlung, welches in dieser Form und diesem Umfang in der österreichischen Forschungsförderung einzigartig ist. Diese Maßnahmen gliedern sich in zwei Bereiche: „Aktivitäten“ umfasst sämtliche Maßnahmen zur Bewusstseinsbildung, Sensibilisierung und Vernetzung sowie zur Sichtbarmachung von Frauen in Forschung und Technologie (Website, Newsletter, Veranstaltungsformate, Preise bzw. Auszeichnungen bis hin zu Datenbank-Rechercheangeboten). „Wissen“ liefert Daten und Informationen, präsentiert Studien und Publikationen sowie thematische Kurzfassungen zu nationalen und internationalen Forschungen in den Themenfeldern „Chancengleichheit“ und „Frauen in FTI“.

Eine detaillierte Darstellung der einzelnen Maßnahmen des Gesamtportfolios sowie der Einschätzungen zum Zielerreichungsgrad ist dem Kapitel 3, „FEMtech Portfolio-Analyse“, sowie insbesondere Kapitel 4, „FEMtech Effekte und Wirkungen“, zu entnehmen.

\section{FEMtech Förderungen}

Gute Effekte erzielt die Förderlinie „Karriere“ hinsichtlich weicher Faktoren der Bewusstseinsbildung in Richtung Chancengleichheit. Strukturelle und organisatorische Veränderungen umfassen v. a. Maßnahmen zur Verbesserung der Work-Life-Balance (v. a. Arbeitszeitflexibilisierung), einer gendersensibilisierten Personalrekrutierung und -entwicklung, in geringem Umfang solche einer expliziten Förderung von Frauen. Ein Niederschlag in konkreten Zahlen (Anstieg des Frauenanteils bei Neueinstellungen und Führungspositionen) ist kaum gegeben. Bei „Karriere“ wird deutlich, dass die Förderung zu einer Dynamisierung im Unternehmen führt: Ohnehin geplante Maßnahmen werden schneller, umfassender und systematischer umgesetzt. In erster Linie konnten für die Umsetzung konkreter Maßnahmen also Unternehmen erreicht werden, in welchen bereits ein Grundverständnis für die Thematik vorhanden war.

Auf „mittlerem“ Niveau erreicht „Karrierewege“ bei den geförderten Kooperationen sein Ziel, vermehrt Karrierevorbereitungsangebote für Studentinnen für die industrielle Forschung zu schaffen. Die Förderlinie trägt so zu einer Angebotsausweitung und Systematisierung der Zusammenarbeit von Unternehmen und Hochschulen in Fragen der weiblichen Nachwuchsgewinnung bei. Der tatsächliche Niederschlag in Form von mehr weiblichen Berufseinsteigerinnen in die Industrie ist ebenfalls „mäßig“, allerdings langfristig auch nur durch eine Kombination von Maßnahmen erreichbar, bspw. durch systematische Veränderungen des Personalrecruitings und strukturelle Veränderungen in Unternehmen, zu denen die im Rahmen von „Karrierewege“ ermöglichten Erfahrungen lediglich anregen können.

„FTI-Projekte“ erzielt auf den verschiedenen Ebenen der Bewusstseinsbildung zur Genderdimension in Forschungsprojekten durchgängig eine Wirkung auf „mittlerem“ Niveau und hat als einzige Förderlinie eine Einreichquote, die deutlich über den tatsächlich bewilligten Projekten liegt. Die erzielten Veränderungen liegen auf geringerem Niveau als bei den beiden anderen Förderlinien. Problematisch ist die zum Teil kritische Außenwahrnehmung, insbesondere bei FTIpolitischen AkteurInnen. Hinzu kommt, dass die Zielgruppe Unternehmen für „Gender in Forschungsprojekten“ erst wenig sensibilisiert zu sein scheint: Sie beteiligen sich in geringerem Ausmaß als bei den beiden anderen Förderlinien; die Projekte wurden zu 55 \% von Universitäten eingereicht. 


\section{FEMtech Aktivitäten}

Mit den unter „Aktivitäten“ gebündelten Formaten zur Sensibilisierung, Bewusstseinsbildung und Vernetzungsförderung in Kombination mit Presse- und Öffentlichkeitsarbeit leistet FEMtech einen hohen Beitrag zur Stärkung der öffentlichen Diskussion zu „Chancengleichheit“ und „Frauen in FTI“. In eingeschränktem Umfang wurde das Ziel der Vernetzung erreicht - hier beschränkt sich die Wirkung auf eine bereits stark themenaffine Community. Ähnlich wie bei „Förderungen“" gilt, dass Unternehmen in der Breite in geringerem Umfang erreicht werden.

Als guter niederschwelliger Einstieg ins Programm und zugleich wichtiger „Backbone“ zur Bindung der engeren Community funktionieren vorrangig Website und Online-Newsletter. Auch die seit Programmstart veranstalteten Netzwerktreffen sind in der gesamten FTI-Community gut bekannt und erreichen ein beachtliches TeilnehmerInnenfeld von rund 1.200 Personen mit hoher Themenaffinität, jedoch starker Konzentration auf Wien. Trotz eines de facto hohen Anteils jeweils neuer TeilnehmerInnen an den einzelnen Terminen bleiben die Treffen in der Außenwahrnehmung von Unternehmen und FTI-politischen AkteurInnen jedoch Treffpunkt für „eine enge Community“.

Beim als Job-, Diplomarbeits- und Praktikabörse angelegten „Forum NaWi)(Tech“ hingegen gibt es wenig Bekanntheit, was sich in der geringen Teilnahme seitens Studentinnen und Absolventinnen widerspiegelt. Entsprechend gering ist die Zufriedenheit auf Seiten der teilnehmenden Unternehmen, die eine stärkere Resonanz auf ihre Präsentation als potenzieller Arbeitgeber erwarten.

Eine starke mediale Resonanz erzielt die Maßnahme „Expertin des Monats“, mit der FEMtech erfolgreich die Präsenz erfolgreicher FTI-Frauen fördert. Die zugrunde liegende „ExpertinnenDatenbank“ profitiert davon jedoch wenig - ihre NutzerInnenschaft beschränkt sich stark auf die Expertinnen selbst bzw. eine enge Community aus Hochschule und Forschung.

\section{FEMtech Wissen}

FEMtech hat mit „Wissen“ einen ausgezeichneten Sammelpunkt für Daten und Fakten, Studien und Publikationen aus dem Bereich Frauen in Forschung und Technologie geschaffen. Sowohl eigene als auch externe Publikationen stehen meist zum Download zur Verfügung. Problematisch ist allerdings, dass hinsichtlich des kleinteilig gegliederten Datenmaterials mittlerweile ein Sättigungsgrad erreicht wurde und die angebotenen Informationen zudem nur von einem kleinen und themenaffinen Kreis, dort jedoch sehr intensiv, genutzt werden. Die Zielgruppe der Unternehmen wird mit „Wissen“ nur marginal erreicht; zudem treffen die Angebote zu wenig deren Bedürfnislage und sind zu wenig zielgruppenspezifisch aufbereitet.

\section{Programmumsetzung und Einbettung von FEMtech}

FEMtech hat in seiner siebenjährigen Geschichte bereits drei unterschiedliche Phasen der Programmschwerpunkte und Organisationsstruktur erlebt, die vierte Phase beginnt mit 2011. Diese Umbrüche waren durch inhaltliche Neukonzeptionen geprägt, die sich entsprechend nachteilig auf Kontinuität und Kohärenz auswirkten. Dass diese Veränderungen von außen nicht als tiefgreifend erlebt wurden, liegt vor allem an der weitgehenden Kontinuität der programmumsetzenden Personen und Organisationen.

Erschwerend wirkten sich strukturelle Faktoren aus: die FEMtech-spezifische organisationale und inhaltliche Kooperation zwischen BMVIT, Kompetenzzentrum und FFG einerseits sowie die Aufteilung der thematischen Verantwortung zwischen drei Ministerien im Rahmen von fFORTE andererseits erhöhen den Abstimmungsbedarf. 
FEMtech bzw. die Thematik der Chancengleichheit und des Gender Mainstreaming im Allgemeinen fanden bisher zwar Aufmerksamkeit, jedoch wenig Anerkennung in der FTI-politischen Community, die sich eher an Fördervolumina und „klassischen“ Programmförderungen orientiert.

\section{Fazit und Schlussfolgerungen}

FEMtech hat die „windows of opportunities“ genutzt, um Chancengleichheit und Frauenförderung im FTI-politischen Bereich aufzugreifen, anzusprechen sowie schnell und zeitgerecht Maßnahmen umzusetzen. Dabei hat die Kombination aus Awareness- mit Fördermaßnahmen funktioniert. Phase 1 eines Mainstreaming-Prozesses von Chancengleichheit (Interessieren) wurde erfolgreich durchgeführt.

- Awareness wurde erreicht, das Thema ist bei den Zielgruppen angekommen. Der FEMtech Bekanntheitsgrad (44 \%) in der FTI-Community ist hoch.

- Die Vernetzung der „Interessierten“ bzw. bereits „Sensibilisierten“ findet erfolgreich statt, der Kreis erweitert sich ständig.

- FTI-politische AkteurInnen bestätigen, dass nun anders über Chancengleichheit in der Forschungsförderung gesprochen wird (mehr Aufmerksamkeit bei Besetzung von Jurys, Boards etc.).

- FEMtech Förderungen haben Showcases geschaffen. Die erfolgreichsten können zukünftig als motivierende Best-Practice-Beispiele zielgruppenspezifisch aufbereitet werden.

- FEMtech NutzerInnen sind in der Regel zufrieden, d. h. die Programmlinien Förderungen, Aktivitäten und Wissen werden gut bewertet.

- Wirkungen entstanden v. a. im Sensibilisierungsbereich, weniger im organisatorischstrukturellen Bereich, kaum im Hinblick auf veränderte Frauenanteile.

Jedoch: FEMtech nutzten bisher überwiegend themenaffine Personen und Organisationen. Es zeigt sich eine übermäßig starke Konzentration auf Wien sowie auf die Bundesländer, die auch sonst in der Forschungsförderung präsent sind.

FEMtech hat sich durch seinen experimentellen Charakter ausgezeichnet. Unterschiedliche Maßnahmen wurden erprobt. Nicht alle davon brachten den erhofften Erfolg, wie die Ergebnisse der Onlinebefragung zeigen (vgl. Kapitel 3). Als wesentlicher Faktor konnte hier die fehlende Bekanntheit der Maßnahmen einerseits („FEMtech meets Fiction“, „Forum NaWi)(Tech“) aber auch mangelnde Klarheit über Ziele und Mehrwert der jeweiligen Maßnahmen identifiziert werden. Hier ist eine Reduktion der Heterogenität der Maßnahmen sowie bessere Kommunikation im Sinne einer Adaptierung auf die Bedarfe der Zielgruppen notwendig, um die vorhandenen Potenziale zu nutzen.

Durch den experimentellen Charakter von FEMtech veränderten sich auch die Zielgruppen: Einzelne Gruppen wurden hinzugefügt, geändert und phasenweise unterschiedlich intensiv betreut. Wenn insbesondere Unternehmen und Forschungseinrichtungen in Richtung Chancengleichheit aktiviert werden sollen, muss differenzierter - sowohl in Kommunikation als auch in Angeboten auf deren Bedarfe und „Hebelmechanismen“ eingegangen werden.

Kritisch und für eine erfolgreiche Weiterführung des Programms hinderlich sind folgende Punkte:

- Die Marke FEMtech ist gut verankert, löst jedoch zu stark die Assoziation „Frauenförderung“ aus. Unternehmen und Forschungseinrichtungen lassen sich jedoch viel mehr über „Chancengleichheit“ erreichen. Hier ist es wichtig, die erfolgreiche Marke inhaltlich klar zu positionieren, damit sie zukünftig nicht als „kommunikative Bremse“ wirkt. 
- Die beiden Hauptzielgruppen der Forschungsförderung sind FTI-Unternehmen und außeruniversitäre Forschungseinrichtungen. Letztere werden mit FEMtech relativ gut erreicht, die Unternehmen aber zu wenig ,abgeholt“.

- FEMtech hat zu wenig in den Aufbau von Kooperations- und Verbundstrukturen investiert. Wenn zukünftig nicht MultiplikatorInnen, FTI-Einrichtungen etc. auf Bundes- und regionaler Ebene als Verbündete gewonnen werden, ist kein substanzieller Fortschritt in Richtung Chancengleichheit zu erwarten.

\section{Empfehlungen}

Um die möglichen Entwicklungsrichtungen von FEMtech zu schärfen, priorisiert das Evaluationsteam abschließend seine Empfehlungen wie folgt:

\section{Schärfung und Konsistenz der gleichstellungspolitischen Ansätze}

- Sowohl in der Kommunikation als auch in der Planung und Umsetzung von Maßnahmen ist es hilfreich, auf die gewählten gleichstellungspolitischen Ansätze klar Bezug nehmen zu können. Während der Frauenförderung das Prinzip der positiven Diskriminierung zugrunde liegt, soll die Förderung der Chancengleichheit Frauen und Männern vergleichbare Erfolgschancen bieten. Entsprechend können Maßnahmen der Chancengleichheit beide Gruppen betreffen bzw. diese einbeziehen (vgl. Kapitel 2.4 „Gleichstellungspolitische Ansätze“).

- Der gewählte Ansatz muss stringent nach außen dargestellt werden, damit die Erwartungen der Zielgruppen daran anschließen können. Dies ist insbesondere in der Kommunikation mit Unternehmen wichtig, für die Überlegungen des wirtschaftlichen Nutzens einer erhöhten Chancengleichheit eine wichtige Rolle spielen.

\section{Konzentration und Fokussierung}

Chancengleichheit setzt strukturelle Veränderungen in Unternehmen und Forschungseinrichtungen voraus. Nicht alle Maßnahmen im Rahmen von FEMtech garantieren eine strukturelle Verankerung der im Rahmen der Förderung erzielten Ergebnisse:

- Die weiterzuführenden Förderlinien „Karriere“ und „FTI-Projekte“ benötigen mehr Service, Beratungs- und Vernetzungsleistungen in Zielgruppenlogik, um hier einen Transfer in die Breite zu erreichen.

- In der Linie „Karrierewege“ ist ein Redesign hin zu einer stärkeren Fokussierung anzudenken. Die Heterogenität der Möglichkeiten (von kurzen Praktika während des Studiums bis zur Dissertation und Unterstützung beim konkreten Berufseinstieg) macht insbesondere die Planung und Umsetzung von Begleitmaßnahmen schwierig.

\section{Regionalisierung und Kooperation}

- Netzwerktreffen, die „Bewerbung“ von Maßnahmen, benötigen über die bereits interessierten Personen hinaus unterschiedliche Angebote für Kooperationen und Verbundstrukturen, speziell mit MultiplikatorInnen und AkteurInnen der Wirtschafts- und Innovationsförderung sowie fachlichen Verbänden auf Bundes- und regionaler Ebene.

Die Stärke von FEMtech liegt u. a. auch in den handelnden Personen. Die ProgrammUmsetzerInnen wurden sowohl von FTI-politischen AkteurInnen als auch von ProgrammNutzerInnen als glaubwürdig, engagiert und professionell empfunden. Der zukünftige Programmerfolg ist jedoch nicht nur von den Programm-UmsetzerInnen zu verantworten, sondern 
im Wesentlichen von der politischen Verantwortlichkeit für Forschung, Technologie und Innovation in Österreich und auch all jenen politischen AkteurInnen, die teilweise oder indirekt zur Rahmengestaltung von Chancengleichheit beitragen. Sie entscheiden über Ernsthaftigkeit und Nachdrücklichkeit, ob Chancengleichheit von Frauen und Männern im österreichischen Innovationssystem bloß eine verbale Zuschreibung oder Selbstverständlichkeit wird. 


\section{Inhaltsverzeichnis}

1 Die FEMtech Programmevaluation ................................................................................ 1

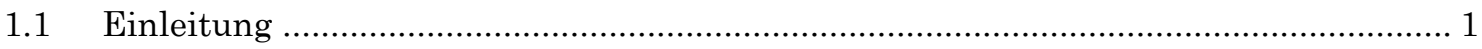

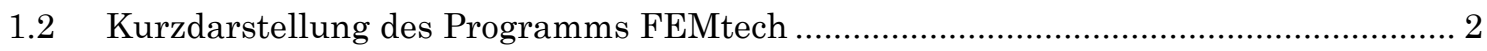

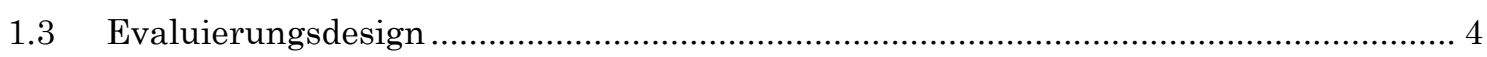

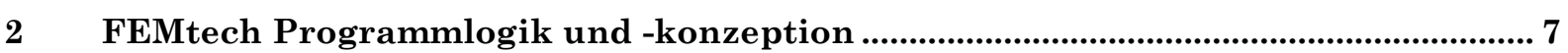

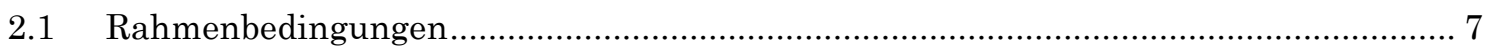

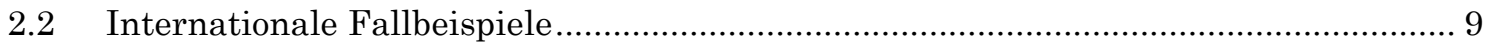

2.2.1 UK Resource Center (UKRC) ………....................................................... 9

2.2.2 Anita Borg Institute for Women in Technology (ABI) ....................................... 10

2.2.3 Mission pour la place des femmes au CNRS (MPDF) ....................................... 11

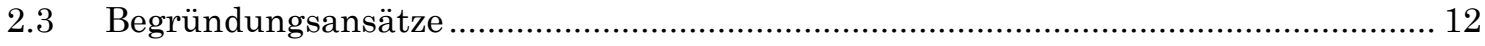

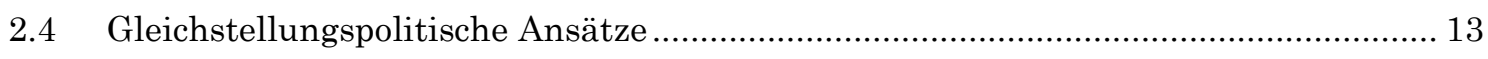

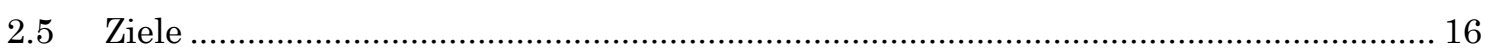

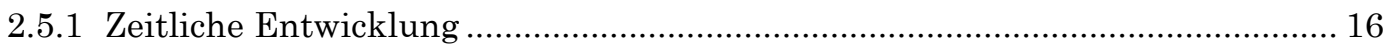

2.5.2 Priorisierung und Zielhierarchie................................................................... 17

2.5.3 Unklarheiten bei Zielen, Strategien und deren Kommunikation ...................... 19

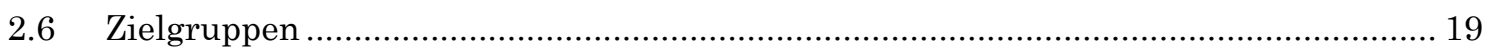

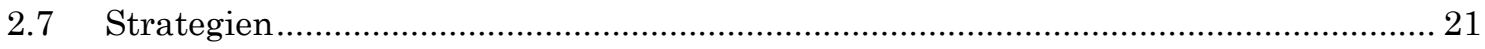

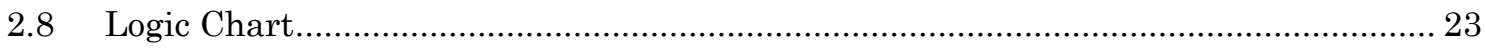

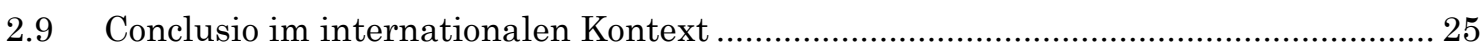

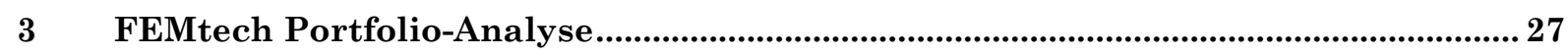

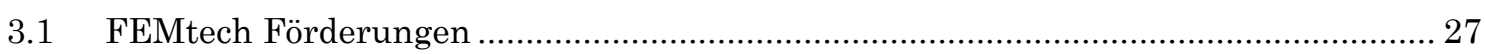

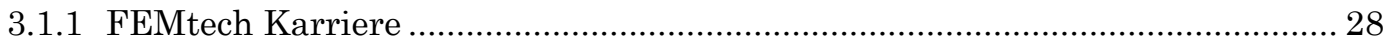

3.1.2 FEMtech Karrierewege ................................................................................. 33

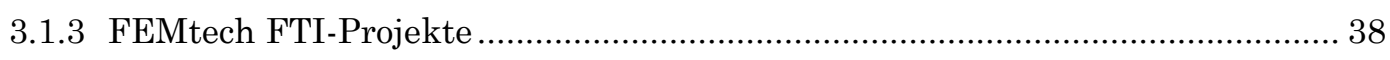

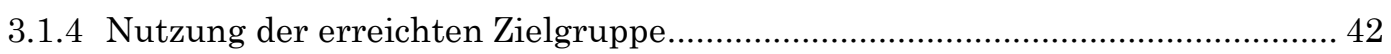

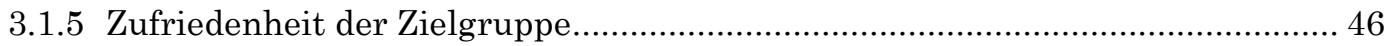

3.1.6 Verbesserungsvorschläge der Zielgruppe ....................................................... 48

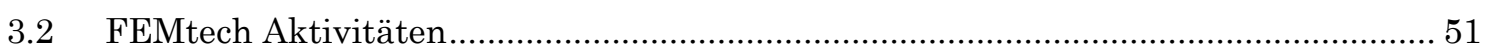

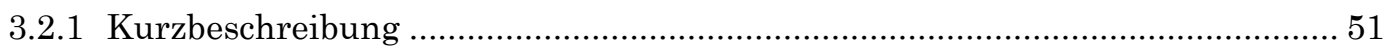

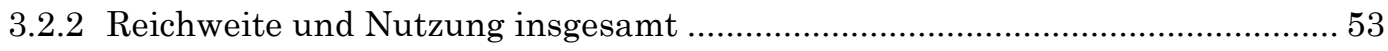

3.2.3 Nutzung und erreichte Zielgruppen je Aktivität ............................................... 55

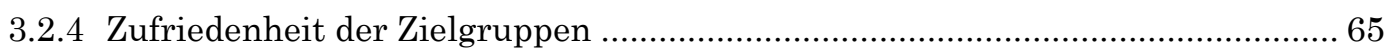

3.2.5 Verbesserungsvorschläge aus Zielgruppensicht .......................................... 66

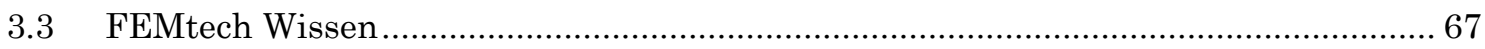

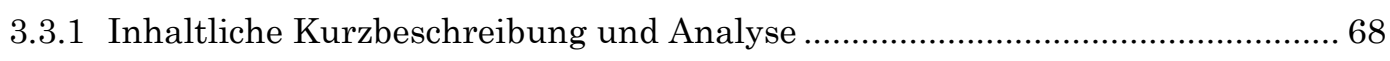

3.3.2 Nutzung (erreichte Zielgruppen) und Zufriedenheit der NutzerInnen .............. 75 
3.3.3 Verbesserungsvorschläge der Zielgruppe ……….......................................... 77

3.4 Maßnahmen für breite Öffentlichkeit und technologiepolitische Community ............. 78

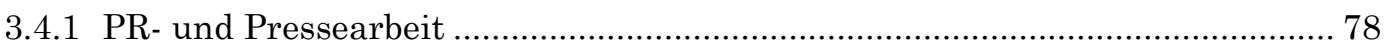

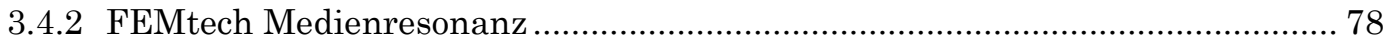

3.4.3 Agenda-Setting in der FTI-Community …………........................................... 82

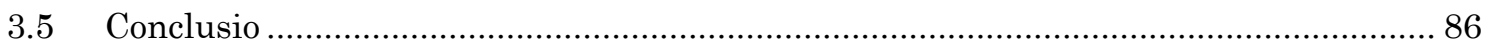

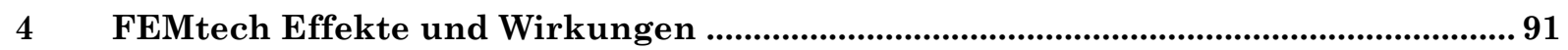

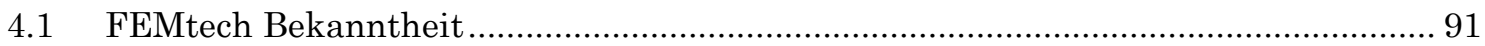

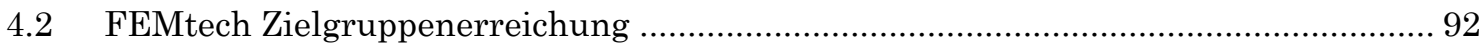

4.3 Durch FEMtech erzielte Veränderungen (Outcomes) ................................................... 97

4.3.1 Outcomes FEMtech Förderungen ...................................................................... 97

4.3.2 FEMtech Wirkungen: Aktivitäten .................................................................... 101

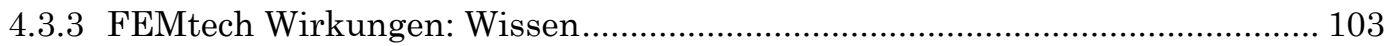

4.3.4 Übergreifende Wirkungen aus Sicht geförderter Frauen.................................. 105

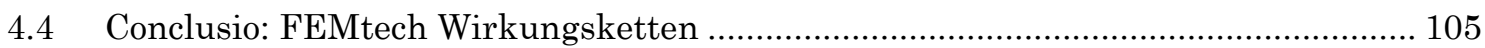

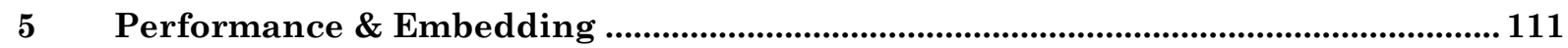

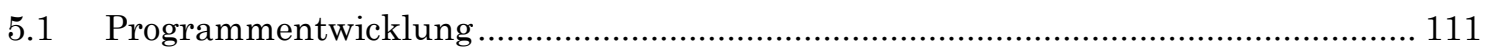

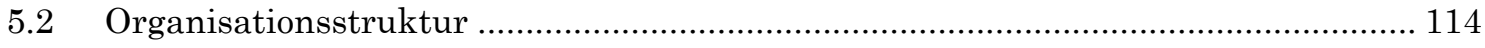

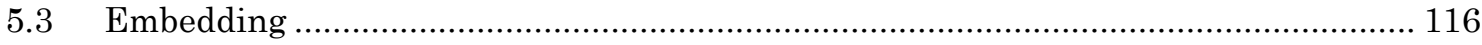

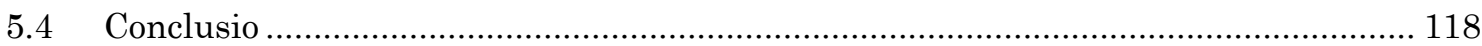

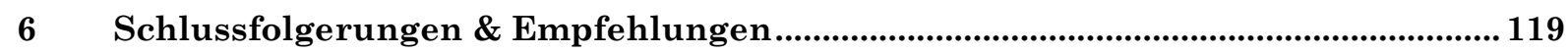

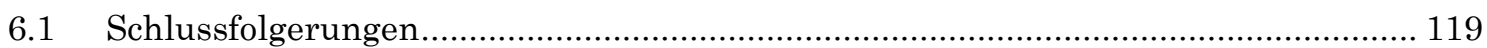

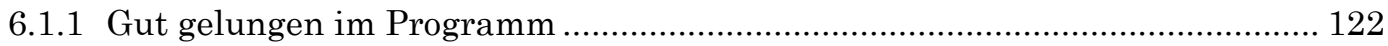

6.1.2 Problematisch für das Programm ………….................................................. 123

6.1.3 Kritisch für das Programm........................................................................... 124

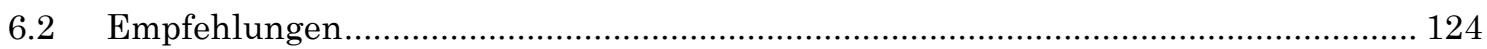

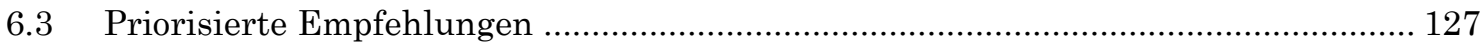

$7 \quad$ Literatur- und Quellenverzeichnis .................................................................................... 129

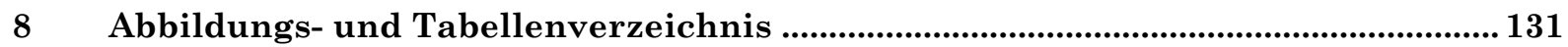

A Anhang: Evaluationsmethoden .................................................................................... 134 


\section{Die FEMtech Programmevaluation}

\subsection{Einleitung}

Mit der Durchführung der FEMtech Programmevaluation im Zeitraum August 2010 bis Ende Jänner 2011 wurde vom Bundesministerium für Verkehr, Innovation und Technologie (BMVIT) die ARGE „3C“ beauftragt. ${ }^{1}$ Die ARGE wurde von den Forschungs- und Beratungseinrichtungen convelop (Graz, Wien), com.X (Bochum) und CEWS, ein Bereich von GESIS, Leibniz-Institut für Sozialwissenschaften (Bonn) eingerichtet. Ziel war es, sämtliche für die Programmevaluation erforderlichen Schlüsselkompetenzen aus einer Hand erhältlich und gebündelt anbieten zu können - mit österreichischem Tiefenwissen und einem perspektivischen „Blick von außen“ angereichert.

Abbildung 1: Partnerorganisationen der ARGE „3C“

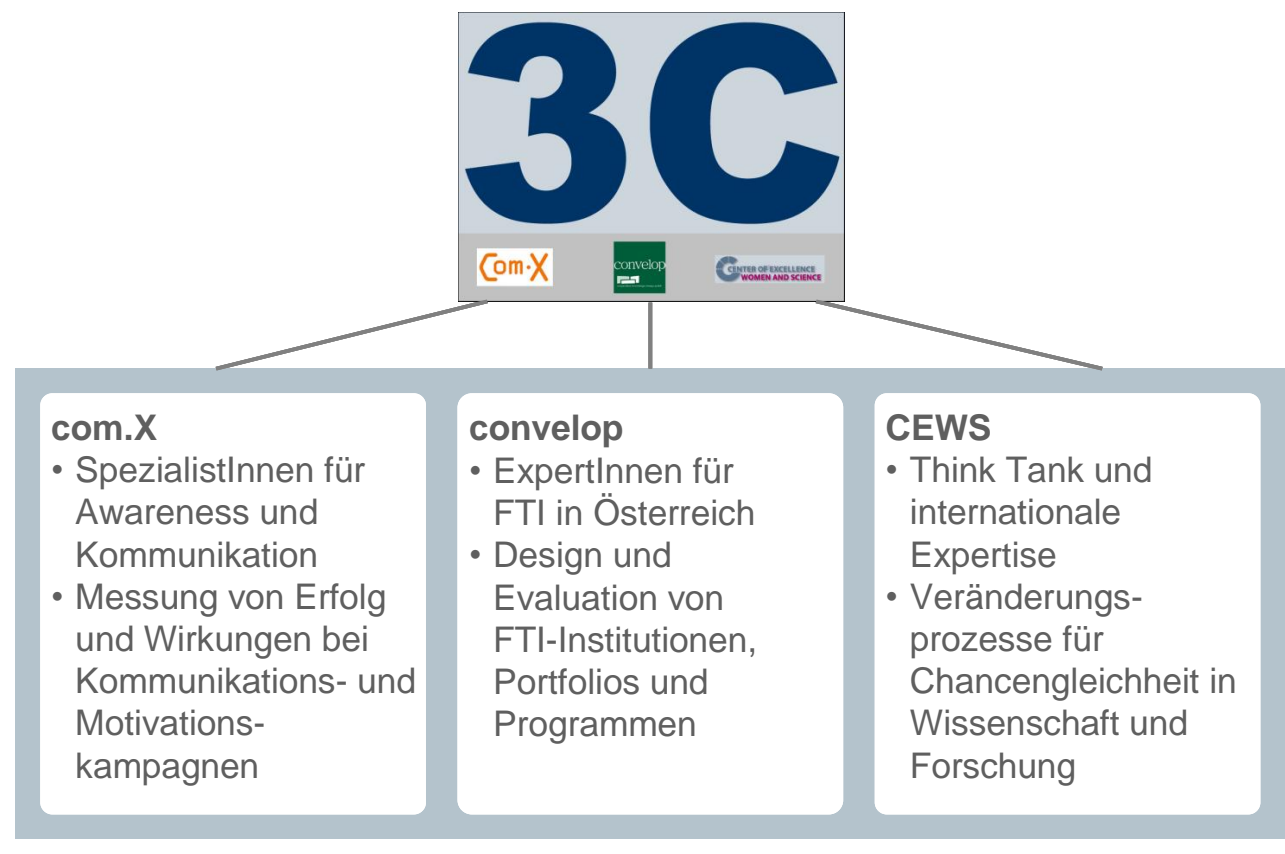

\section{FEMtech Programmevaluation}

Quellenangabe: ARGE „3C“

\footnotetext{
${ }^{1}$ Im Rahmen eines zweistufigen Verhandlungsverfahrens wurde die ARGE „3C“ als Bestbieterin ermittelt.
} 
Die FEMtech Programmevaluation weist einen breiten Ansatz mit vielfältigen Erhebungsbereichen im Vergleich zu Standardevaluierungen von FTIProgrammen auf. Dies begründet sich aus folgenden Programmgegebenheiten:

1. FEMtech ist kein „klassisches Förderprogramm“, sondern besteht aus einem vielfältigen Maßnahmenbündel mit mehreren Programmlinien zur Erhöhung der Anzahl von Frauen in der industriellen Forschung und in außeruniversitären Forschungseinrichtungen. Derart vielschichtig und breit ist kein anderes Programm in der österreichischen Forschungsförderung aufgestellt.

2. Mit FEMtech hat das BMVIT in Österreich eine Awareness- und Sensibilisierungsschiene in der Forschungsförderung eröffnet, die es bis dato in diesem Umfang nicht gab. Dem Spektrum an Informations- und Kommunikationsmaßnahmen wurde in der Evaluierung besondere Aufmerksamkeit geschenkt, da hier einerseits für FEMtech selbst zentrale Ergebnisse liegen, andererseits dem Thema „Wirkung von Awarenessmaßnahmen“ in der technologiepolitischen Community besonderes Interesse entgegengebracht wird.

3. FEMtech wird nicht exklusiv von der Österreichischen Forschungsförderungsgesellschaft abgewickelt (wie die meisten FTI-Programme), sondern bedient sich für die Awareness- und Wissensangebote eines Kompetenzzentrums, bestehend aus vier eigenständigen Unternehmen. Diese Form des geteilten Wissens und ihr Zusammenwirken wurden in der Programmbewertung mit erfasst.

Herausfordernd für die Evaluation war der Zeitraum: In einem halben Jahr Bearbeitungszeit erfolgte die umfassende Evaluierung unter Einbeziehung vielfältiger AkteurInnen und Unmengen an Materialien. Der ARGE „3C“ war es ein Anliegen, bereits vom ersten Tag der Beauftragung inhaltlich voll durchstarten zu können, damit für den Zwischenbericht nach drei Monaten und den Endbericht nach sechs Monaten die Ergebnisse vorliegen. Die Zeit drängte, denn mit Jahresbeginn 2011 sollten die FEMtech Programmorganisation und die -inhalte neu aufgesetzt werden.

\subsection{Kurzdarstellung des Programms FEMtech}

FEMtech ist ein 2003 initiiertes Programm des Bundesministeriums für Verkehr, Innovation und Technologie (BMVIT) zur Förderung der Chancengleichheit in Forschung und Technologie. ${ }^{2}$

Das Programm wird im Rahmen von fFORTE (Frauen in Forschung und Technologie) durchgeführt, einer auf Empfehlung des Rats für Forschung und Technologieentwicklung im Februar 2002 ins Leben gerufenen ministerienübergreifenden Initiative. Neben dem BMVIT sind an fFORTE beteiligt (in namensalphabetischer Reihenfolge): das Bundesministerium für Unterricht, Kunst und Kultur, das Bundesministerium für Wissenschaft und Forschung und das Bundesministerium für Wirtschaft, Familie und Jugend. Jedes der Ministerien verantwortet ein Programm (neben FEMtech fFORTE academic,

${ }^{2}$ Grundlagen für dieses Kapitel sind: Endbericht FEMtech Kompetenzzentrum vom Juli 2010, FEMtech Website 2010 sowie fForte-Website 2010. 
w-fFORTE und fFORTE Schule); insgesamt sollen dadurch Mädchen und Frauen während aller Phasen des Bildungsweges begleitet und Karrierehindernisse entlang der beruflichen Laufbahn abgebaut werden.

FEMtech setzt in diesem Kontext in der „späteren“ Phase des Berufsorientierungs- und Karriereplanungsprozesses an. Das Programm zielt in erster Linie darauf ab, Rahmenbedingungen und Karrierechancen für bereits berufstätige Frauen und (potenzielle) Berufseinsteigerinnen in Unternehmen und Organisationen der industriellen und außeruniversitären Forschung zu verbessern. Dadurch soll die Attraktivität technischer und naturwissenschaftlicher Berufe für Frauen gesteigert werden, um insgesamt die Anzahl weiblicher Beschäftigter zu erhöhen. Die Unternehmen und Organisationen profitieren umgekehrt von qualifizierten und motivierten Mitarbeiterinnen, weiblichen Sichtweisen in gemischt-geschlechtlichen Teams und damit der Realisierung von mehr Innovationspotenzial (detailliert beschäftigt sich Kapitel 2 „FEMtech Programmlogik und -konzeption“ mit Zielen und Zielgruppen).

Seit Mitte 2008 bis Ende 2010 lag die Durchführung des Programms beim FEMtech Kompetenzzentrum in Kooperation mit der Österreichischen Forschungsförderungsgesellschaft (FFG). Das Kompetenzzentrum ist vertreten durch die Österreichische Gesellschaft für Umwelt und Technik (ÖGUT), Joanneum Research Forschungsgesellschaft, Bohmann Druck und Verlag sowie D\&Z Consulting (siehe dazu im Detail Kapitel 5 „Performance \& Embedding“).

Die Basis von FEMtech bildet der Bereich „Förderungen“, dessen Abwicklung bei der FFG liegt. Er umfasst die drei Programmlinien Karriere, Karrierewege und FTI-Projekte (letztere beiden sind 2008 neu hinzugekommen). Gefördert werden Maßnahmen zur Verbesserung der Chancengleichheit innerhalb von Unternehmen und außeruniversitären Forschungseinrichtungen (Karriere), Kooperationen zwischen Hochschulen und Unternehmen zur Karrierevorbereitung von Studentinnen in der industriellen Forschung (Karrierewege) sowie Forschungsprojekte mit Gender-Dimension (FTI-Projekte). Antragsberechtigt und damit direkte Zielgruppe sind je nach Förderlinie (im Detail siehe dazu Kapitel 3.1 „FEMtech Förderungen“) forschungs- und technologieintensive Unternehmen, außeruniversitäre Forschungseinrichtungen, Hochschulen sowie in einer Programmlinie auch EinzelforscherInnen.

FEMtech ist jedoch nicht ausschließlich „Förderprogramm“, sondern besteht darüber hinaus aus einem vielfältigen Maßnahmenbündel zur Bewusstseinsschaffung und Sensibilisierung, Vernetzung und Wissensvermittlung, welches in dieser Form und diesem Umfang in der österreichischen Forschungsförderung einzigartig ist. Sämtliche Maßnahmen außerhalb der standardmäßigen Förderungsabwicklung liegen zwischen 2008 und Ende 2010 in der Hand des Kompetenzzentrums und gliedern sich in zwei Bereiche:

„Aktivitäten“ umfasst sämtliche Maßnahmen zur Bewusstseinsbildung, Sensibilisierung und Vernetzung sowie zur Sichtbarmachung von Frauen in Forschung und Technologie. Dazu kommt ein breites Set unterschiedlicher Formate zum Einsatz: über Kommunikationsinstrumente (Website, Newsletter), Veranstaltungsformate, Preise bzw. Auszeichnungen bis hin zu DatenbankRechercheangeboten (siehe dazu im Detail Kapitel 3.2 „FEMtech Aktivitäten“). 
Begleitet und in die Öffentlichkeit „transportiert“ werden die Aktivitäten durch PR- und Pressearbeit.

„Wissen“ liefert Daten und Informationen, präsentiert Studien und Publikationen sowie thematische Kurzfassungen zu nationalen und internationalen Forschungen im Themenfeld „Chancengleichheit“ und „Frauen in FTI“ (siehe dazu im Detail Kapitel 3.3 „FEMtech Wissen“). Ziel ist es, zentrale themenspezifische Informationen aufzubereiten und leicht zugänglich zu machen. Präsentiert werden sämtliche Produkte dieses Bereichs auf der FEMtech Website.

\subsection{Evaluierungsdesign}

Die formative Evaluation wurde während der Laufzeit des FEMtech Programms durchgeführt. Ihre Funktion besteht in der Bewertung der mittelfristigen Effekte und Wirkungsrichtungen (bei einer Laufzeit von ca. 7 Jahren) des Programms auf struktureller Ebene. Die Zielsetzung ist, bisherige Maßnahmen zu bewerten und insgesamt einen Beitrag für die Weiterentwicklung des Programms zu leisten. Das Evaluationsdesign sollte den umfassenden Zielsetzungen, Zielgruppen und dem Maßnahmenspektrum gerecht werden und zugleich der Kombination aus Aktivitäten, Förderungen und Wissen in FEMtech entsprechende Aufmerksamkeit schenken.

Abbildung 2: Design Programmevaluation FEMtech

Start: Mitte Juni 2010

Ende: Mitte Dez. 2010

1. AP 1 Konzept | Programmlogik, Programmkonzeption, Rahmenbedingungen, Ziele, Zielgruppen, Entwicklung und Positionierung FEMtech

2. AP 2 Performance | Strukturen und Prozesse, FEMtech-Leistungsportfolio, Organisationsstruktur und Kooperation

3. AP 3 Wirkung | Vertiefende Evaluierung

Analyse Aktivitäten
Konzeption
Umsetzung
Zielerreichung

Analyse Förderungen
Konzeption
Umsetzung
Zielerreichung

Analyse Wissen

Konzeption

Umsetzung

Zielerreichung

Nutzen-/Wirkungsanalyse

4. AP 4 Embedding | Einbindung und Funktion FEMtech für österreichische (insb. industrielles/außeruniversitäres) FTI-System, Einbettung Frauenförderung und internationaler Konnex

5. AP 5 Bewertung | Zusammenfassender Befund, Schlussfolgerungen, Empfehlungen für die Weiterentwicklung von FEMtech.

Evaluationsbericht inkl. grafischer Aufbereitung der Daten.

Erläuterung: AP = Arbeitspaket, $\mathrm{Q}=$ Querschnittsbereich

Quellenangabe: ARGE „3C“

Das Ziel der FEMtech Programmevaluierung ist die Analyse der bisherigen Maßnahmen auf unterschiedlichen Ebenen durch die Analysen seines Leistungs- und Produktportfolios als auch der Feststellung des Nutzungsverhal- 
tens und der Veränderungsbereitschaft der direkten Zielgruppen (Wirkungsbereiche). Die Gewinnung einer Perspektive von außen wird durch den Vergleich mit drei internationalen Fallbeispielen im Bereich Chancengleichheit für Frauen in Forschung und Technologie ermöglicht.

Der besondere Dank gilt den Verantwortlichen im BMVIT sowie der FFG und des Kompetenzzentrums. Die Bemühung, alle erforderlichen Daten und Informationen bereitzustellen, war sehr groß. Eine Vielzahl von Unterlagen standen zur Analyse für die zentralen Aussagen und Schlussfolgerungen zur Verfügung. Einige Dokumente und Daten (Leistungsverträge für den gesamten Zeitraum), Nutzungszahlen (z. B. Website-Visits) für die Jahre 2006 und 2007, Aufzeichnungen über Downloads oder sonstige Nutzungszahlen aus dem Bereich „Wissen“ konnten leider nicht bereitgestellt werden, da sie teilweise nicht vorhanden sind bzw. nicht erhoben werden.

Der Dank der EvaluatorInnen gilt auch den zahlreichen InterviewpartnerInnen und den befragten Personen im Rahmen der Onlineumfrage: Hier war die Antwortbereitschaft äußerst groß, sodass Aussagen bestens fundiert sind. Eine genaue Aufstellung der Eckpunkte (Anzahl der Befragten, Rücklaufquoten etc.) der verschiedenen Befragungen sind im Anhang nachzulesen.

Um der Komplexität des FEMtech Programms bei Wahrung der Forschungsökonomie gerecht zu werden, kam ein zielführender Mix aus quantitativen und qualitativen Methoden zum Einsatz. ${ }^{3}$ Gleichzeitig entstand so eine Erhebungsvielfalt im Sinne einer Triangulation. So lassen sich einerseits fundierte Aussagen zu Größenordnungen gewinnen und andererseits tiefer liegende Wirkmechanismen erklären. Folgender Überblick verdeutlicht, welche Methoden dies im Einzelnen sind, welche Zielgruppen bzw. Gegenstandsbereiche adressiert werden und auf welche Arbeitspakete sie einzahlen:

${ }^{3}$ vgl. Kapitel A „Anhang: Evaluationsmethoden“ 
Abbildung 3: Maßnahmenmix FEMtech Programmevaluation

\begin{tabular}{|c|c|c|c|c|c|c|c|}
\hline & Methode & $\begin{array}{l}\text { AP1 } \\
\text { Konzept }\end{array}$ & $\begin{array}{l}\text { AP2 } \\
\text { Performance }\end{array}$ & $\begin{array}{l}\text { AP3 } \\
\text { Wurikung }\end{array}$ & $\begin{array}{l}\text { AP4 } \\
\text { Embedding }\end{array}$ & $\begin{array}{l}\text { AP5 } \\
\text { Bemertung }\end{array}$ & Q1 \\
\hline Dokumentenanalysen in allen Arbeitspaketen & & $\checkmark$ & $\checkmark$ & $\checkmark$ & $\checkmark$ & $\checkmark$ & $\checkmark$ \\
\hline \multicolumn{8}{|l|}{$\begin{array}{l}\text { PROGRAMMZIELBEREICHE } \\
\text { Unternehmen-Frauen-Awareness }\end{array}$} \\
\hline Onlinebefragung / Forschung u. Industrie & & & & $\checkmark$ & & & \\
\hline Interviews | oberes Management & & $\checkmark$ & & $\checkmark$ & $\checkmark$ & & \\
\hline Onlinebefragung | FEMtech Nutzende & & & & $\checkmark$ & & & \\
\hline Fokusinterviews | FEMtech Fördernehmerinnen & k? & $\checkmark$ & & $\checkmark$ & & & \\
\hline Nutzungsanalyse | FEMtech Website & 뭉 参? & & & $\checkmark$ & & & \\
\hline Medienresonanzanalyse / FEMtech in Medien & & & & $\checkmark$ & & & \\
\hline Produktanalyse I FEMtech Output & (2) & & $\checkmark$ & $\checkmark$ & $\checkmark$ & & $\checkmark$ \\
\hline Interviews | FTI-Akteure & k\& & $\checkmark$ & & & $\checkmark$ & & \\
\hline \multicolumn{8}{|l|}{$\begin{array}{l}\text { PROGRAMMUMSETZUNG } \\
\text { BMVIT + Kompetenzzentrum + FFG }\end{array}$} \\
\hline Programmgespräch | FEMtech UmsetzerInnen & inth & $\checkmark$ & $\checkmark$ & & $\checkmark$ & & \\
\hline Self-Assessement | FEMtech Umsetzerlnnen & $\dot{\mathbf{1}}_{2}^{2}$ & & $\checkmark$ & & & $\checkmark$ & $\checkmark$ \\
\hline Fokusinterviews| ehem. Programmleiterinnen & kil & $\checkmark$ & $\checkmark$ & & $\checkmark$ & & \\
\hline Fallbeispiele u. Analysen | International & iiil & $\checkmark$ & $\checkmark$ & $\checkmark$ & $\checkmark$ & $\checkmark$ & $\checkmark$ \\
\hline
\end{tabular}

Quellenangabe: ARGE „3C“

Der umfassende Evaluationsansatz erlaubt es, Aussagen über Wahrnehmung und Wirkung einzelner Leistungen und Produkte für die Zielgruppen sowie in der Öffentlichkeit generell (Medien) zu treffen. Er geht jedoch deutlich darüber hinaus: Betrachtet wird das gesamthafte Zusammenwirken, die Konzeption und Ausrichtung, um dabei die Effekte und Wirkrichtungen von FEMtech zu erkennen. Die Evaluation stellt damit einen wesentlichen Beitrag zur strategischen Steuerung und Positionierung von FEMtech dar. Für die Programmverantwortlichen (BMVIT) erfolgt eine Bewertung bisheriger Leistungen und Wirkungen sowie handlungsleitender Perspektiven und Gestaltungsinformationen über das Jahr 2010 hinaus. 


\section{FEMtech Programmlogik und -konzeption}

Die Analyse der Programmkonzeption erfolgt auf der Grundlage der folgenden zur Verfügung stehenden Quellen:

- Programmdokument, Sonderrichtlinie FEMtech fFORTE, 2007

- FEMtech Website, 2010

- Jahresberichte, 2003-2009

- Flyer, 2003-2008

Das Programmkonzept von 2004 sowie die Leistungsverträge lagen nicht vor.

Auf der Grundlage der Dokumentenanalyse, der Logic-Chart-Analyse, der Programmgespräche und der Interviews mit den FTI-AkteurInnen, den Interviews mit dem oberen Management und den Fokus-Interviews wird im Folgenden die Programmlogik von FEMtech rekonstruiert. Darüber hinaus wird die Programmkonzeption mit drei internationalen Fallbeispielen verglichen: dem UK Resource Center for Women in Science (UKRC) ${ }^{5}$ in Großbritannien, der Mission pour la place des femmes au CNRS (MPDF) ${ }^{6}$ in Frankreich und dem Anita Borg Institute for Women and Technology (ABI) ${ }^{7}$ in den USA.

\subsection{Rahmenbedingungen}

Auslöser für die Initiierung des Programms FEMtech durch das Bundesministerium für Verkehr, Innovation und Technologie (BMVIT) im Jahr 2003 war die Tatsache, dass Österreich an letzter Stelle der EU 15 rangierte - mit einem Frauenanteil in Industrie und Forschung von lediglich 9 \% (Stand 1999). Um die Wettbewerbsfähigkeit des Wirtschaftsraumes zu garantieren, ist eine höhere Partizipation von Forscherinnen auf nationaler und europäischer Ebene ebenso wie eine exzellente Forschung mit Gender-Dimension notwendig. Angesichts der Wachstumsdynamik in Forschung und Entwicklung erzeugte die schlechte Positionierung Österreichs bei der Beteiligung von Forscherinnen in technologieorientierten Bereichen einen hohen Handlungsdruck, der auch zur Etablierung des Programms FEMtech führte.

Sowohl auf EU-Ebene als auch in den Mitgliedsstaaten liegen zahlreiche Studien vor, die sich mit den Wirkmechanismen, die die Integration von Frauen in Wissenschaft und Forschung behindern, auseinandersetzen. Die europäische ExpertInnen-Gruppe WIR stellte 2003 für den Bereich der industriellen Forschung spezifische Barrieren für die Teilhabe von Forscherinnen fest und sprach Empfehlungen aus. Bei der internationalen Konferenz „Women in Industrial Research - Speeding up Changes in Europe“ wurden die folgenden

\footnotetext{
${ }^{4}$ Stand: 21.01 .2011

${ }^{5} \mathrm{http} / / / \mathrm{www}$. theukrc.org/

${ }^{6} \mathrm{http} / / /$ www.cnrs.fr/mpdf/

${ }^{7}$ http://anitaborg.org/
} 
fünf Empfehlungen ausgesprochen (European Commission 2004; European Commission 2003c):

- Unternehmen, Schulen und Hochschulen müssen NachwuchsWissenschafterinnen zu einer Karriere in der Industrieforschung motivieren.

- Unternehmen müssen Frauen fördern, die Forschungskultur ändern, die Rahmenbedingungen ändern und mehr Frauen in Führungspositionen bringen.

- Die Anzahl und die Partizipation von Frauen im Innovationsprozess muss durch Awareness-Maßnahmen gefördert werden.

- Die Wissensgrundlage über Frauen in der Industrieforschung muss verbessert werden.

- Frauen in der Industrieforschung müssen sichtbarer und mächtiger werden und das öffentliche Bild der Industrieforschung muss sich ändern.

Die Europäische Kommission stellte 2005 als Herausforderungen für die Zukunft fest:

- Steigerung der Anzahl von Frauen in Entscheidungspositionen in Forschung und Technologie

- Vereinbarkeit von beruflichem und privatem Leben

- Gender und wissenschaftliche Exzellenz

- Stärkung der Genderforschung

- Erhöhung der Partizipation von Frauen in FTI

Seit den 1990er-Jahren initiierten die europäischen Nationalstaaten und die Europäische Kommission zahlreiche Maßnahmen und Programme, um die Beteiligung von Frauen in Wissenschaft und Forschung zu erhöhen (European Commission 2008; Rees 2002). Gegenüber Programmen in der Schweiz und in Deutschland mit ihrem Fokus auf Hochschulen (Spreyermann / Rothmayr 2008; BLK 2007; Löther / Mühlenbruch 2004) ist das österreichische Dachprogramm fFORTE - „Frauen in Forschung und Technologie" mit der Adressierung aller Durchführungssektoren in seiner großen Breite der Zielgruppen im deutschsprachigen Raum einzigartig. Trotz zahlreicher gleichstellungspolitischer Maßnahmen in Wissenschaft und Forschung auf europäischer und nationalstaatlicher Ebene wächst der Forscherinnenanteil in den letzten 10 Jahren jedoch nur langsam: In der außeruniversitären Forschung stagniert der Frauenanteil in Österreich seit 2004 bei rund 21 \% (FEMtech (o.J.a)). In der Industrieforschung stieg der Frauenanteil von 1999 bis 2006 um 5 Prozentpunkte von $9 \%$ auf $14 \%$, im Hochschulsektor von 2000 bis 2006 immerhin um 9 Prozentpunkte auf $35 \%$ (European Commission 2009; European Commission 2003a).

Einerseits besteht ein hoher Wissensstand über die Ursachen der Unterrepräsentanz von Wissenschafterinnen. Europaweit wird die Notwendigkeit, dass mehr Wissenschafterinnen an Forschung und Innovation teilhaben, anerkannt. Zahlreiche gleichstellungspolitische Projekte wurden initiiert. Andererseits sind nur langsame Veränderungen bei der Integration von Forscherinnen zu 
verzeichnen. Vor diesem Hintergrund besteht dringender Bedarf, die Wirkungen und Optimierungsoptionen von Programmen und Maßnahmen zu analysieren.

\subsection{Internationale Fallbeispiele}

Um die Programmlogik von FEMtech in einen internationalen Rahmen einzuordnen, werden dem Programm drei internationale Fallbeispiele gegenübergestellt: das UK Resource Center (UKRC), die Mission pour la place des femmes au CNRS (MPDF) und das Anita Borg Institute for Women and Technology (ABI).

\subsubsection{UK Resource Center (UKRC)}

Das UKRC ist die führende Service-, Strategie- und Beratungseinrichtung der britischen Regierung hinsichtlich der Unterrepräsentanz von Frauen in den MINT8-Fächern. Ziel ist die Gleichstellung von Frauen und Männern in Naturund Ingenieurwissenschaften, Technologie und Raumplanung. Servicestellen sind in ganz Großbritannien regional verteilt. Das Portfolio des UKRC umfasst Beratung, Training, Auszeichnungen, Wissenstransfer, Strategieentwicklung und Vernetzung. Die Angebote richten sich einerseits an Frauen auf allen Karrierestufen und andererseits an eine Vielzahl von AkteurInnen, wie z. B. ArbeitgeberInnen, Bildungseinrichtungen, Fachverbände, Frauenorganisationen und -netzwerke und die Regierung.

Abbildung 4: UKRC

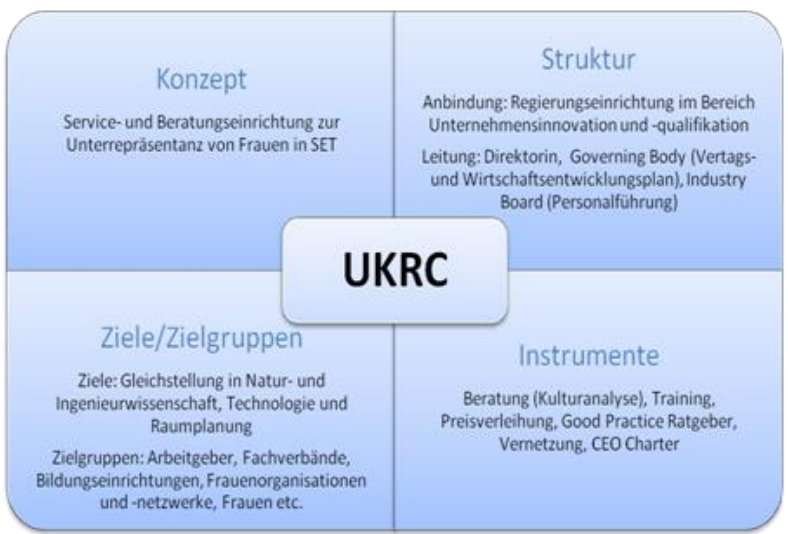

Quellenangabe: ARGE „3C“
Die Webseite des UKRC bietet neben einem Zugang zu den Angeboten für Frauen und Organisationen einen News-Service, ein Informationsportal mit Zahlen und Hintergrundwissen, einen Blog sowie einen Veranstaltungskalender.

Das UKRC wird finanziert vom Department for Business Innovation and Skills und geleitet in Partnerschaft mit dem Bradford College. Weitere Partner sind die University of Cambridge, University of Oxford, Open University, Sheffield Hallam University und Queen Mary University of London. Die Direktorin des UKRC wird durch den Governing Body bei der wirtschaftlichen Führung

des UKRC unterstützt.

\section{Zitat}

„The UKRC Vision: By 2030 we will have an environment in UK employment, research and policy-making in science, engineering, technology and the built environment, in which women contribute to, participate in and share the benefit equally to their male counterparts.

\footnotetext{
${ }^{8}$ MINT $=$ Mathematik, Informatik, Naturwissenschaft, Technik
} 
This long-term vision can only be achieved if all stakeholders within science, engineering and technology take the necessary action to make it happen. "

\section{Wesentliche Merkmale}

Das UKRC hat ein sehr breites Angebot für vielfältige Zielgruppen und ist daher am besten mit FEMtech vergleichbar. Es unterscheidet sich von FEMtech insbesondere durch den Schwerpunkt auf Beratung, Kulturanalyse, Training und Strategieentwicklung und durch eine stärkere Anbindung an die Wissenschaft. Die Vision der Erreichung von Chancengleichheit im Jahr 2030 kann nach eigener Einschätzung nur erreicht werden, wenn die EntscheidungsträgerInnen die notwendigen Maßnahmen ergreifen.

Ein interessantes Instrument ist die Entwicklung der CEO Charter, die Organisationen einen öffentlichkeitswirksamen Rahmen für ihr Bekenntnis zur Förderung von Frauen im MINT-Bereich bietet. Im Gegensatz zu FEMtech sind die Angebote teilweise auch regional verfügbar.

\subsubsection{Anita Borg Institute for Women in Technology (ABI)}

Das ABI ist ein gemeinnütziges Institut im US-amerikanischen Bundesstaat Kalifornien, das seit 1997 sowohl den Einfluss von Frauen auf alle Aspekte von Technologie als auch den positiven Einfluss der Technologie auf die Welt der Frauen fördert. Ziel ist die Förderung der Einstellung, Bindung und Entwicklung von Frauen im Technologiesektor. Die vom ABI aufgelegten Programme haben einen Schwerpunkt auf der Vernetzung von Frauen untereinander und auf die Auszeichnung von Frauen, Teams und Einrichtungen.

Abbildung 5: Anita Borg Institute

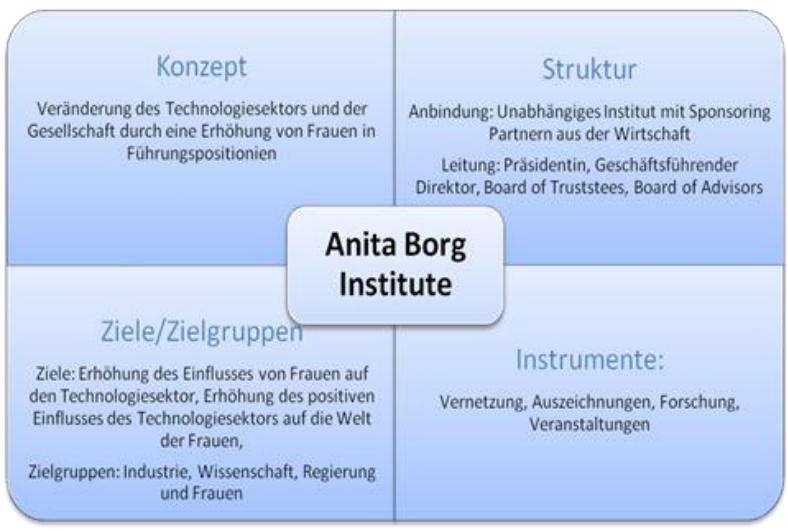

Quellenangabe: ARGE „3C“
Die Webseite des ABI bietet neben der Darstellung der Programme ein Informationsportal sowie Wissenswertes zur Kooperation, Unterstützung von und zur Vernetzung mit dem ABI.

Finanziert wird das ABI durch Spenden und durch Sponsoring großer Unternehmen, wie z. B. Google, Microsoft, SAP, Amazon etc.

\section{Zitat}

„By providing inclusive platforms designed to ensure women's voices, ideas and spirits will result in higher levels of technical innovation, $A B I$ delivers programs that are changing the world for women and for technology. "10

\section{Wesentliche Merkmale}

Die Strategie des ABI ist geprägt vom Ansatz des „Virtuous Cycle“: Frauen sollen ermutigt werden, ingenieurwissenschaftliche und technische Berufe auszuüben und darin Karriere zu machen. Hierdurch sollen sie Einfluss auf die

\footnotetext{
${ }_{9}$ Quelle: http://www.theukrc.org/about-us (letzter Zugriff: 5. April 2011)

${ }^{10}$ Quelle: http://anitaborg.org/about/mission/ (letzter Zugriff: 5. April 2011)
} 
Entwicklung kreativer Technologien bekommen. Über die daraus entstehenden Produkte und Dienstleistungen sollen die Einsatzmöglichkeiten von Frauen auf dem globalen Markt widergespiegelt und langfristig ein Berufsfeld geschaffen werden, das Frauen unterstützt.

Schwerpunkt der Aktivitäten von ABI sind Auszeichnungen, Vernetzung und Veranstaltungen.

\subsubsection{Mission pour la place des femmes au CNRS (MPDF)}

Seit 2001 ist die MPDF eine von fünf Stabsstellen des Präsidenten innerhalb der größten französischen staatlich geförderten Forschungsorganisation CNRS. Ziel der Mission ist die Förderung der beruflichen Gleichstellung innerhalb des CNRS, der Genderforschung und von Genderaspekten in der Forschung sowie die Sichtbarmachung von Frauen und die Sensibilisierung von SchülerInnen. Es bestehen Kooperationen und Vernetzung innerhalb des CNRS sowie auf nationaler, europäischer und internationaler Ebene.

Abbildung 6: CNRS

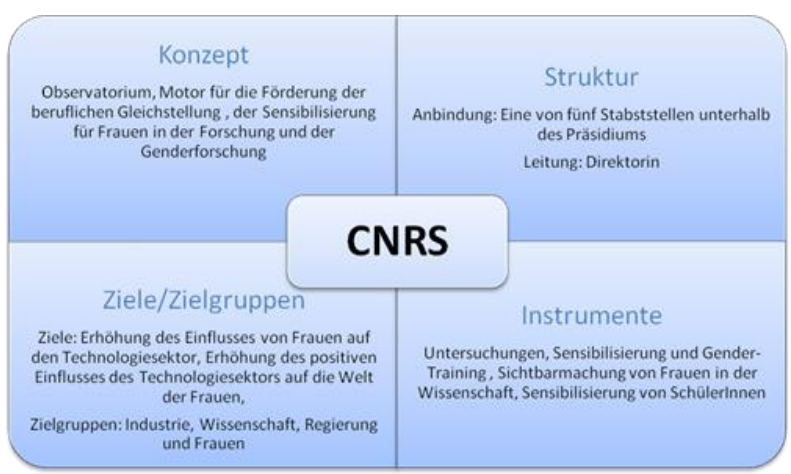

Quellenangabe: ARGE „3C“
Die Webseite enthält kaum Informationen über die Umsetzung und Durchführung der Aktivitäten. Die Publikationslisten sind ähnlich aufgebaut wie bei FEMtech, enthalten aber in der Regel nur ein Abstract und keine Möglichkeit zum Download.

Über die finanzielle Ausstattung liegen keine Informationen vor. Einschließlich der Direktorin sind insgesamt neun Mitarbeiterinnen für die Mission tätig.

\section{Zitat}

„Sensitization and training to gender issues: in collaboration with the Human Resources Direction, the then Department of Physics and Mathematics at CNRS, and the Labor Market and Gender European Research Group, the Mission created an original pedagogical instrument: the Multidisciplinary scientific workshops. "11

\section{Wesentliche Merkmale}

Hervorzuheben ist ein Kooperationsprojekt der Mission mit allen Instituten des CNRS, INSHS und INSB zur Erfassung der nationalen Forscherinnen und Forscher zu Gender und/oder Frauen. Die Ergebnisse sind über eine Datenbank im Internet verfügbar, sollen die Sichtbarkeit des Forschungsbereichs erhöhen und eine Bestandsaufnahme ermöglichen.

Ebenso interessant ist der Ansatz der pluridisziplinären wissenschaftlichen Workshops, bei denen WissenschafterInnen zu einem Gender/Gleichstellungsthema zunächst Vorträge hören und dann in Workshops/Arbeitsgruppen das Wissen diskutieren und vertiefen. Eine Reihe dieser Veranstaltungen wurde 2002-2004 von der Mission in Zusammenarbeit mit

\footnotetext{
${ }^{11}$ Quelle: http://www.cnrs.fr/mpdf/ (Letzter Zugriff: 5. April 2011)
} 
einem wissenschaftlichen Organisationskomitee und weiteren Partnern durchgeführt.

\subsection{Begründungsansätze}

Aus der Dokumentenanalyse lassen sich für die Programmkonzeption drei wesentliche Begründungsansätze für FEMtech ermitteln, die sich an den europäischen Empfehlungen der Expertinnengruppe „Women in Industrial Research“ orientieren (European Commission 2004; European Commission 2003b).

\section{Humanressourcen}

Im Mittelpunkt stehen die Probleme des bestehenden und steigenden Bedarfs an technischem und naturwissenschaftlichem Personal innerhalb der österreichischen Forschungs- und Unternehmenslandschaft, der Rekrutierung von Nachwuchswissenschafterinnen und des auf niedrigem Niveau stagnierenden Frauenanteils im Bereich FTI insbesondere bei den Führungspositionen (Stichwort „Humanressourcen“).

FEMtech argumentiert hier aus dem Blickwinkel der Wirtschaft und der Unternehmen. Die Attraktivität des Berufsfeldes sowie die Strukturen und Arbeitsbedingungen im FTI-Bereich, auch im Hinblick auf die Vereinbarkeit von beruflichem und privatem Leben werden nicht als Begründungsansätze herangezogen, sondern als Mittel zum Zweck gesehen, um die Anzahl der Frauen zu erhöhen und damit die fehlenden Humanressourcen auszugleichen. Mit diesem Fokus reagierte FEMtech auf die schlechte Position Österreichs im europäischen Vergleich. Seine Legitimation bezog das Programm FEMtech aus dem Verweis auf die Nutzung des weiblichen Innovationspotenzials und die Rekrutierungsproblematik. Langfristig greift dieser Ansatz jedoch zu kurz, da die Erhöhung des Frauenanteils von zahlreichen Faktoren (z. B. Arbeitsmarktpolitik und -entwicklung) abhängt und von einzelnen Programmen kaum beeinflussbar ist. Auch Strukturveränderungen sind nur langsam zu erreichen, garantieren aber eine nachhaltige Impulswirkung im FTI-Bereich.

\section{Sichtbarmachung, Sensibilisierung und Bewusstseinsbildung}

Als zweite Problematik wird die geringe Präsenz und Sichtbarkeit von Frauen im Bereich Forschung und Technologie sowie das mangelnde Bewusstsein und die fehlende Sensibilisierung bezüglich des Themas herausgestellt (Stichwort: „Sichtbarmachung, Sensibilisierung und Bewusstseinsbildung“).

Ein herausragendes positives Merkmal von FEMtech ist es, Frauen und ihre Unterrepräsentanz in Forschung und Technologie in einer breiten Öffentlichkeit zum Thema zu machen. Die positive Sichtbarmachung von Frauen in FTI, die Vernetzung untereinander sowie die Sensibilisierung der FTI-AkteurInnen für die Problematik der Unterrepräsentanz sind eine wichtige Voraussetzung für die langfristige Erhöhung des Frauenanteils. Hierdurch kann bei den Unternehmen, den außeruniversitären Forschungseinrichtungen, den tertiären Bildungseinrichtungen und der FTI-politischen Community die Problemwahrnehmung initiiert und der Handlungsbedarf aufgezeigt werden (Sensibilisierung). Mit der Sichtbarmachung erfolgreicher Frauen können Vorbilder für 
Nachwuchswissenschafterinnen präsentiert und Frauen motiviert werden, in Führungspositionen aufzusteigen. Die Bewusstseinsbildung schließlich, die aus der Sensibilisierung für Frauen in FTI und ihre Unterrepräsentanz folgen kann, ist eine entscheidende Voraussetzung für Veränderungsprozesse. Die Wichtigkeit von „Awareness-Building“ wird auch in einer Studie der EUKommission zu Frauen in der industriellen Forschung bestätigt, in der festgestellt wurde, dass die Unternehmen eine sogenannte „neutrale“ Personal- und Rekrutierungspolitik betreiben, die in der Wirkung jedoch Frauen ausschließt (European Commission 2004). Es besteht in Unternehmen vielfach kein Bewusstsein für die indirekte Diskriminierung von Frauen und umfassende gleichstellungspolitische Ansätze wie Gender Mainstreaming sind nicht bekannt. Daher ist es ein wichtiges Ziel, Führungskräfte und Personalverantwortliche zu sensibilisieren und ihre Gender-Kompetenz zu erhöhen.

FEMtech hat den Aspekt der Sichtbarmachung, Sensibilisierung und Bewusstseinsbildung in erster Linie mit dem Programmbereich „Aktivitäten“ aufgegriffen und - wie die Online-Befragung von forschungs- und technologieintensiven Organisationen im Rahmen dieser Evaluation bestätigt (vgl. Kapitel 4 „FEMtech Effekte und Wirkungen") - erfolgreich umgesetzt. Bewusstseinsbildung im Sinne der Förderung von Gender-Kompetenz und Gender-Wissen bei Führungskräften und Personalverantwortlichen in den Unternehmen und außeruniversitären Forschungseinrichtungen wird zwar nicht explizit als Programmziel kommuniziert, aber implizit über die Voraussetzungen und Anforderungen für die Förderung von FEMtech Karriere-Projekten transportiert. Auch der Bereich „Wissen“ knüpft an den Ansatz der Sensibilisierung und der Bewusstseinsbildung an. Eine breite Wissensbasis über Frauen in FTI soll dazu beitragen, das Thema in die Öffentlichkeit zu bringen, den Handlungsbedarf zu verdeutlichen und passgenaue Strategien zu entwickeln.

\section{Forschung mit Gender-Dimension}

Der dritte Problembereich ist schließlich die mangelnde Berücksichtigung der unterschiedlichen Sichtweisen und Denkansätze von Frauen und Männern in den Forschungsinhalten, was sich sowohl auf die Qualität als auch auf die Akzeptanz der Forschung und Produkte auswirkt (Stichwort „Forschung mit Gender-Dimension"). Hier greift FEMtech neben dem rein quantitativen Ansatz der Erhöhung des Frauenanteils den qualitativen Ansatz der GenderDimension in der Forschung auf.

Das Förderkonzept zur Beachtung der Gender-Dimension in Forschungsprojekten und die Gestaltung einer eigenen Förderlinie wurde - laut Programmgesprächen mit den FEMtech UmsetzerInnen - auf Basis einer Studie von Prof. Dr. rer. nat. Martina Schraudner, „Gender als Innovationspotenzial in Forschung und Entwicklung“, entwickelt. Die diesbezüglichen Ausführungen im Programmdokument sind - im Vergleich zu den vorher genannten Bereichen - nur knapp gehalten.

\subsection{Gleichstellungspolitische Ansätze}

Den oben genannten Begründungsansätzen liegen unterschiedliche gleichstellungspolitische Ansätze zugrunde. In den Dokumenten zur Programmkonzep- 
tion von FEMtech werden diese Ansätze jedoch nicht hergeleitet bzw. definiert. Weiters sind die verwendeten Begriffe und deren inhaltliche Konkretisierung nicht konsistent.

\section{Definition gleichstellungspolitischer Ansätze}

Als Ausgangsverständnis der Begriffe der gleichstellungspolitischen Ansätze liegen dieser Evaluation folgende Definitionen zugrunde:

Chancengleichheit: Formal sollen die Zugangshindernisse zu Bildung, Arbeit etc. abgebaut werden, um allen Personen die gleichen Ausgangsbedingungen zu ermöglichen. Es handelt sich hier um das Ändern von Rahmenbedingungen. Weitreichender und auch im Kontext von FEMtech zu verstehen ist eine substanzielle Chancengleichheit. Diese ergänzt die formale Chancengleichheit so weit, dass nicht nur die Ausgangsbedingungen gleich sind, sondern auch vergleichbare Erfolgschancen aller Gruppen vorzufinden sind. Dieser Ausgleich der Erfolgschancen und damit die Förderung der Chancengleichheit kann beispielsweise durch gezielte Förderung von Männern oder Frauen erfolgen. (vgl. Pimminger et.al. 2009, S.4 f)

Frauenförderung: Frauenförderung liegt das Prinzip der positiven Diskriminierung zugrunde. Es werden Maßnahmen forciert, welche gezielt Frauen fördern und einen Ausgleich der bestehenden Benachteiligung von Frauen erwirken wollen, ohne ganzheitlichen Blick auf die Auswirkungen auf beide Geschlechter. Es werden hier keine Veränderungen der Rahmenbedingungen und Strukturen erwirkt. (vgl. Bergmann u. Pimminger 2004, S.21)

Gender Mainstreaming: Rahmenbedingungen in Unternehmen, bei Entscheidungen und Planung etc. sind so gestaltet, dass die Bedürfnisse und Ansprüche beider Geschlechter berücksichtigt werden. Es werden mögliche Auswirkungen auf Männer und Frauen bewusst wahrgenommen und berücksichtigt. Dies unterstützt bei der Förderung der geschlechtlichen Gleichstellung und zeigt Lücken und Brennpunkte auf, welche einer spezifischen Förderung für Frauen oder Männer bedürfen. (vgl. Bergmann u. Pimminger 2004, S.20f)

\section{Bezugnahme zu gleichstellungspolitischen Ansätzen bei FEMtech}

In den ausgewerteten Quellen werden Begriffe wie Chancengleichheit, Frauenförderung und Gender Mainstreaming verwendet, jedoch an zentraler Stelle nicht erläutert oder definiert. Einzig eine Definition von Gender Mainstreaming findet sich versteckt in einem Dokument der Antragsunterlagen mit dem Hinweis „Was ist unter Genderkompetenz im Rahmen von FEMtech zu verstehen? Hier erfahren Sie mehr dazu." In den Programm-Flyern ab 2005 werden Maßnahmen den Konzepten Chancengleichheit, Frauenförderung und Sensibilisierung zugeordnet, ohne dass diese definiert werden. Die Zuordnung ist zudem teilweise problematisch: So wird z. B. die Einstellung von mehr Frauen als Maßnahme der Frauenförderung aufgelistet, nicht als Maßnahme der Chancengleichheit. Jedoch fördern die Bemühungen von Unternehmen, bei Stellenbesetzungen verstärkt auf Bewerbungen und auf das Potenzial von Frauen zu achten, nicht die einzelne Bewerberin. Vielmehr dienen diese Maßnahmen dazu, eine ausgewogene Personalstruktur zu erreichen und die Unterrepräsen- 
tanz von Frauen abzubauen und sind somit dem gleichstellungspolitischen Ansatz der Chancengleichheit zuzuordnen. Dementsprechend wird die gezielte Personalrekrutierung von Frauen im FEMtech Flyer auch unter Chancengleichheit aufgeführt.

Es überrascht, dass FEMtech nicht ausführlicher die verschiedenen gleichstellungspolitischen Ansätze beschreibt, da eine differenzierte Kommunikation dieser Ansätze eine wichtige Grundlage für die Sensibilisierung und Bewusstseinsbildung ist. Vergleichbare Institutionen wie beispielsweise das GenderKompetenzZentrum (Berlin) bieten auf ihren Webseiten sogenannte „GenderGlossare“, die AkteurInnen, die mit den Konzepten und Begrifflichkeiten nicht vertraut sind, eine Wissensbasis bieten. Hier könnte FEMtech einen wichtigen Beitrag zur Verbesserung des Gender-Wissens leisten.

Obwohl FEMtech in erster Linie den Begriff Chancengleichheit verwendet, stehen in der Konzeption der Programmbereiche Maßnahmen der Frauenförderung im Vordergrund. So werden verschiedene gleichstellungspolitische Ansätze bedient, ohne dass diese benannt oder zueinander in Beziehung gesetzt werden. Diese Schwerpunktsetzung bei den konkreten Programminhalten führt dazu, dass die FTI-AkteurInnen FEMtech vorwiegend als Frauenförderungsprogramm wahrnehmen (vgl. Kapitel 4.1 „FEMtech Bekanntheit von FEMtech"). Der teilweise nach außen intransparente Umgang mit den gleichstellungspolitischen Konzepten spiegelt die Akzeptanzprobleme wider, die gleichstellungspolitische Programme und Maßnahmen regelmäßig haben.

Die Programm-UmsetzerInnen tendieren dazu, unabhängig von dem dahinterstehenden gleichstellungspolitischen Konzept den Begriff Chancengleichheit nach außen zu verwenden, da sie von einer höheren Akzeptanz seitens der Unternehmen und Forschungseinrichtungen ausgehen. Die Interviews mit FTI-AkteurInnen (vgl. Kapitel 3.4.3 „Agenda-Setting in der FTI-Community“) bestätigen diese Annahme. Andererseits werden die Begriffe nicht „trennscharf" wahrgenommen, sondern auch mit Frauenförderung assoziiert.

In den konzeptionellen Dokumenten von FEMtech findet sich unabhängig von den verwendeten Begrifflichkeiten ein Schwerpunkt auf den Ansätzen der Chancengleichheit und der Frauenförderung. Dagegen verwenden die Beschreibungen der Programmbereiche sprachlich überwiegend den Begriff Chancengleichheit. Inhaltlich werden jedoch die Konzepte der Frauenförderung (Frauen gezielt unterstützen, Verbesserung ihrer beruflichen Position), der Chancengleichheit (Rahmenbedingungen verändern) und des Gender Mainstreamings (Rahmenbedingungen sollen den Bedürfnissen von Frauen und Männern gleichermaßen entsprechen) angesprochen. Im Bereich „Förderung“ ist für die Linie „Karriere“ eine Kombination von Chancengleichheitsund Frauenförderansatz festzustellen. Die 2007 eingeführte Programmlinie „Karrierewege“ stellt hingegen allein auf die Förderung von Nachwuchswissenschafterinnen ab. Die FTI-Projekte schließlich legen einen GenderMainstreaming-Ansatz für Forschungsprojekte zugrunde.

Die Vermischung der gleichstellungspolitischen Ansätze „Frauenförderung“ und „Chancengleichheit“ wird z. T. auch intern von den ProgrammUmsetzerInnen kritisch bewertet. Der vorrangige Bezug auf Chancengleichheit stehe aus pragmatischen Gründen im Vordergrund, da dieser Ansatz in Unter- 
nehmen am ehesten anschlussfähig sei (vgl. Kapitel 3.4.3 „Agenda-Setting in der FTI-Community“).

\subsection{Ziele}

Da die Terminologie in den verschiedenen Quellen bezüglich der Ziele und Strategien nicht durchgängig einheitlich ist, werden die Ziele im Rahmen der Evaluation wie folgt kategorisiert und in eine Zielhierarchie gebracht:

- Leitziele: langfristig, global, nicht direkt messbar

- Mittlerziele: aus Leitzielen abgeleitet, Handlungsansätze vorgebend, mittelfristig, aber nicht hinreichend operationalisierbar und damit messbar

- Handlungsziele: aus Leit- und Mittlerzielen abgeleitet, quantitativ oder qualitativ formulierte Vorgaben, die auf der Ebene von Einzelprojekten zu einem bestimmten Zeitpunkt in einer bestimmten Qualität erreicht werden können

Der Begriff „Leitziel“ entspricht in der Terminologie von FEMtech der Mission des Programms. Die Unterteilung in Leit-, Mittler- und Handlungsziele entspricht in etwa der Systematik der allgemeinen, spezifischen und operationalen Ziele.

\subsubsection{Zeitliche Entwicklung}

In den Zielen von FEMtech lässt sich eine Verschiebung feststellen, von einem Fokus auf der quantitativen Erhöhung des Frauenanteils und dem AwarenessAnsatz hin zu einem Schwerpunkt auf den Rahmen- und Zugangsbedingungen für die Ausbildung und die Berufe im FTI-Bereich. Die zeitliche Entwicklung der Ziele lässt sich in erster Linie anhand der Jahresberichte und der Programmflyer im Zeitraum von 2003-2008 nachvollziehen. In den ersten Berichten liegt der Fokus auf der Erhöhung der Anzahl der Frauen in FTI und auf der Bewusstseinsbildung und Sensibilisierung. Das Thema "Gender" in der Forschung ist noch kein Ziel. Hinsichtlich der Strukturierung und Gewichtung der Ziele findet dann mit dem Jahresbericht für 2007 eine wesentliche Änderung statt, die anhand der folgenden Tabelle verdeutlicht wird: 
Tabelle 1: Ziele in den Jahresberichten

2005, 2006

- Verbesserung der Anzahl und Situation von Frauen in Forschung und Technologie

- Verstärkung der Präsenz

- Verbesserung der Karrierechancen

- Steigerung der Attraktivität technischer Ausbildungen und forschungsund technologierelevanter Berufe für Frauen

- Bewusstseinsbildung bei betroffenen Frauen in Unternehmen und Forschungseinrichtungen

- Sensibilisierung und Thematisierung
2007, 2008, 2009

- Erhöhung des Anteils an Wissenschafterinnen und Technikerinnen im Bereich Forschung, Technologie und Innovation (FTI)

- Erhöhung des Frauenanteils in Führungspositionen und Verbesserung des Zugangs zu Führungspositionen

- Verbesserung der Situation und der Karrierechancen von Frauen in Unternehmen und in der außeruniversitären Forschung

- Verbesserung der Strukturen, Rahmen- und Zugangsbedingungen für die Beschäftigung von Frauen in Forschung und Technologie

- Verstärkte Ausrichtung von Forschungsinhalten an die Bedürfnisse und Interessen von Frauen (ab 2008 ergänzt durch Sensibilisierung von WissenschafterInnen für das Thema „Gender“)

- Aufbau von Nachwuchswissenschafterinnen und -technikerinnen für forschungs- und technologieintensive Unternehmen (Industrie und KMU)

- Vertiefung und Stärkung der Kooperationen zwischen Bildungs- und Ausbildungseinrichtungen und Unternehmen

Der Schwerpunkt (drei von sieben Zielen) verlagert sich 2007 von der Bewusstseinsbildung und Sensibilisierung auf die Verbesserung der Strukturen und Rahmenbedingungen in den Unternehmen und der außeruniversitären Forschung sowie der Zugangs- und Karrierechancen von Frauen in Forschung und Technologie. Während „Gender in der Forschung“ nunmehr als Ziel genannt wird, fällt der Bereich „Bewusstseinsbildung und Sensibilisierung“ (Programmbereich „Aktivitäten“) in den Jahresberichten 2007 und 2008 bei der Auflistung der Ziele vollständig weg. Das Thema „Sensibilisierung“ ist lediglich im Jahresbericht 2008 für den Bereich „FTI-Projekte“ in Ergänzung zur verstärkten Ausrichtung von Forschungsinhalten auf die Bedürfnisse und Interessen von Frauen thematisiert worden (,Sensibilisierung von WissenschafterInnen für das Thema „Gender““). Auch in den Programmflyern von 2003 bis 2008 lassen sich diese Veränderungen bei der Priorisierung der Ziele ablesen.

\subsubsection{Priorisierung und Zielhierarchie}

Die Verschiebung der Ziele von der Erhöhung des Frauenanteils durch Frauenfördermaßnahmen zur Veränderung der Rahmenbedingungen in den Unternehmen und Forschungseinrichtungen entspricht der Entwicklung der gleich- 
stellungspolitischen Ansätze von „fixing the women“ zu „fixing the organisation“. Die ursprüngliche Ausrichtung auf die Erhöhung des Frauenanteils wurde nicht weiter verfolgt, sondern es wurde erkannt, dass Chancengleichheit nicht allein durch eine Hebung des Anteils an Frauen in Forschung und Technologie erreicht werden kann, sondern die Veränderung der Rahmenbedingungen in den Unternehmen notwendig ist. Diese Veränderung wird auch von den Programm-UmsetzerInnen und FTI-politischen AkteurInnen reflektiert: „Köpfezählen kann es nicht sein" (Zitat PG3). Mit der veränderten Priorisierung der Ziele hat FEMtech flexibel auf Erkenntnisse während der Laufzeit des Programms reagiert. Gleichzeitig besteht ein hoher politischer Druck, den Frauenanteil im FTI-Bereich zu erhöhen, da das schlechte Abschneiden Österreichs im EU-Vergleich die Motivation zur Einrichtung von FEMtech war. In diesem Spannungsfeld ist es nachvollziehbar, dass FEMtech die Veränderung in der Zielhierarchie nicht so deutlich nach außen kommuniziert.

Weiters spiegelt das derzeitig geringere Gewicht des Bereichs „Sensibilisierung, Bewusstseinsbildung und Sichtbarmachung“ wider, dass dieser Ansatz bisher erfolgreich war und nun durch weitergehende Ansätze abgelöst werden muss.

Die Verschiebung der Ziele findet jedoch keinen Niederschlag in den aktuellen Publikationen (Jahresberichte ab 2008, Flyer 2008) und auf der Website. Dort lassen sich vielmehr zum momentanen Zeitpunkt (Januar 2010) die folgenden Ziele identifizieren:

\section{FEMtech Zielhierarchie}

\section{Leitziel:}

- Erhöhung des Frauenanteils im Bereich FTI, insbesondere auf der Ebene der Führungspositionen

\section{Mittlerziele:}

- Erhöhung der Attraktivität technischer Ausbildungen und forschungs- und technologierelevanter Berufe für Frauen

- Gewinnung von Nachwuchswissenschafterinnen für Karrieren in F\&Eintensiven Unternehmen und außeruniversitären Forschungseinrichtungen

- Bewusstsein für Gender und Frauen in FTI

- Gender in der Forschung

Die konkreten Handlungsziele, die mit den Programmlinien und -bereichen von FEMtech verfolgt werden, lassen sich wie folgt zusammenfassen:

- Verbesserung der Rahmenbedingungen, der Situation und der Zugangs- und Karrierechancen von Frauen in Unternehmen der industriellen und außeruniversitären Forschung

- Aufbau, Förderung und Begleitung von Nachwuchswissenschafterinnen

- Akzeptanz und Interesse der beteiligten Forscherinnen und Forscher für das Thema „Gender" in Forschungsprojekten erhöhen

- Frauen in FTI und ihr Potenzial sichtbar machen 
- Vernetzung fördern

- Informationen zum Thema „Frauen in FTI“ aufbereiten und zugänglich machen sowie Wissen zum Thema „Gender“ und „Frauen in FTI“ erhöhen

\subsubsection{Unklarheiten bei Zielen, Strategien und deren Kommunika- tion}

In den Dokumenten von FEMtech werden die Ziele in allgemeine, explizite und spezielle Ziele unterteilt. Dabei werden jedoch Ziele und Strategien zum Teil nicht klar abgegrenzt. So wird beispielsweise das Ziel der Bewusstseinsbildung, Sensibilisierung und Sichtbarmachung von Frauen in FTI als Strategie dargestellt, um Veränderungen in den Strukturen und Kulturen von Forschung und Technologie zu erreichen. Gleichzeitig lassen sich die Bereiche „Bewusstseinsbildung“ und „Veränderung von Strukturen“ jedoch auch als zwei gleichrangige Ziele identifizieren, die durch den Bereich „Förderung“ (Programmlinie Karriere) und „Aktivitäten“ innerhalb von FEMtech verfolgt werden sollen.

Die oben genannten Ziele werden nach außen und nach innen nicht einheitlich präsentiert und mit unterschiedlicher Priorisierung kommuniziert. In der Kommunikation der Ziele liegt der Fokus insgesamt auf den Themen „Förderung der Chancengleichheit“, „Erhöhung des Frauenanteils“, „Verbesserung der Rahmenbedingungen“ und „Verbesserung der Karrierechancen von Frauen“. Die Ziele in den Bereichen „Gender in der Forschung“ sowie „Sensibilisierung“ und „Bewusstseinsbildung“ werden weniger prominent platziert.

\subsection{Zielgruppen}

Die Beschreibung der Zielgruppen des Programms FEMtech ist über die siebenjährige Entwicklung nicht eindeutig durchführbar. Seit Beginn des Programms ist eine Verschiebung der in den Unterlagen genannten Zielgruppen erkennbar, wobei sich hier eindeutig das Verständnis für das Wort Zielgruppe, auch durch die Erweiterung der Angebote und Bereiche, mitentwickelt hat. $\mathrm{Zu}$ Beginn wurden vor allem Frauen, welche in FTI tätig sind und langfristig von den Förderungen profitieren sollen, als Zielgruppe bezeichnet. In der weiteren Entwicklung des Programms wurden Frauen in FTI zunehmend durch die einreichberechtigten Organisationen in FTI als Zielgruppe im Bereich Förderungen ersetzt. Der Fokus verlagerte sich also hin zu den einreichberechtigten Organisationen, in denen die strukturellen Änderungen durchgeführt werden, welche eine Förderung der Chancengleichheit verfolgen. Die Mischung der Begriffe Zielgruppe/Einreichberechtigte zeigt sich auch auf der Website innerhalb des Programmbereichs „Förderungen“. In der Auflistung der jeweils Einreichberechtigten sind diese mit dem Titel „Zielgruppe“ überschrieben. Im Folgenden wird zwischen direkten Zielgruppen (Einreichberechtigte und AkteurInnen, bei denen eine strukturelle Änderung hin zu mehr Chancengleichheit erreicht werden soll) und indirekten Zielgruppen (AkteurInnen, die langfristig von den Veränderungen profitieren - Frauen in FTI) unterschieden. Diese Unterscheidung soll auch als Erläuterung für die Verwendung des Zielgruppenbegriffs innerhalb dieses Evaluierungsberichts dienen. Anzumerken ist 
in den Bereichen Aktivitäten und Wissen, dass eine solche Trennung in direkte und indirekte Zielgruppen aufgrund der Breite der Maßnahmen nicht möglich ist.

Als indirekte Zielgruppen können alle Frauen in FTI (Studentinnen, Nachwuchswissenschafterinnen, Führungskräfte, Graduierte) identifiziert werden.

Direkte Zielgruppen lassen sich wie folgt mit absteigender Gewichtung darstellen:

- Technologieintensive Unternehmen

- Außeruniversitäre Forschungseinrichtungen

- Tertiäre Ausbildungseinrichtungen mit technisch-naturwissenschaftlichem Schwerpunkt

- FTI-politische Community (bei Aktivitäten und Wissen)

- EinzelforscherInnen und Einzelforscher (nur in der Linie FTIForschungsprojekte)

Innerhalb der Förderlinien stellen sich die indirekten und direkten Zielgruppen wie folgt dar: Die Programmlinie „Karrierewege“ beispielsweise zielt direkt auf die Vernetzung von Hochschulen und Unternehmen ab, um damit geeignete Rahmenbedingungen zu schaffen, welche für die geförderten Maßnahmen dienen sollen, die die Entwicklung des weiblichen Nachwuchses zum Ziel haben. Auch in der Programmlinie „Karriere“ werden Projekte finanziert, die Rahmenbedingungen in Unternehmen schaffen, um die Forscherinnen und Technikerinnen in ihrer beruflichen Karriere zu unterstützen bzw. die besonderen Bedürfnisse und Interessen von Frauen in der Forschung zu berücksichtigen. In der Programmlinie „FTI-Forschungsprojekte“ beispielsweise soll eine Veränderung des Bewusstseins von WissenschafterInnen durch die Förderung von Forschungsprojekten mit Gender-Dimension erreicht werden, um die unterschiedlichen Bedürfnisse von Männern und Frauen in den Forschungsthemen genauer wahrzunehmen und eine nachhaltige „Awareness“, insbesondere in Bezug auf Anforderungen von Frauen, auch für zukünftige Projekte zu schaffen. Hier werden auch Einzelpersonen und ihre Forschungsprojekte als direkte Zielgruppe angesprochen. Diese starke Ausrichtung auf Frauen als indirekte Zielgruppe entspricht der Selbstwahrnehmung der Programmverantwortlichen und ergibt sich ebenso aus den Interviews mit den FTIAkteurInnen.

Die durch die unterschiedlichen FEMtech Maßnahmen avisierten Zielgruppen sind sehr heterogen. Eine klare Trennung von Einreichberechtigten für Förderungen und Zielgruppen, welche von den durch die Förderung möglichen Maßnahmen und Initiativen profitieren sollen, wäre hilfreich. Eine weitere Differenzierung der „profitierenden“ bzw. indirekten Zielgruppen nach Ausbildungsstatus kann helfen, die Strategien und Maßnahmen gezielt auf deren Bedürfnisse anzupassen. Beispielsweise sind die Bedürfnisse von Studierenden vor und nach Abschluss eines Diploms bzw. einer Dissertation hinsichtlich der weiteren Karriereplanung sehr unterschiedlich.

Aber auch Unternehmen bzw. Organisationen haben, je nach Größe und Zielsetzung, unterschiedliche Ausgangs-Voraussetzungen, um Maßnahmen zur 
Erhöhung der Chancengleichheit umzusetzen. Erarbeitetes Wissen über Maßnahmen für Unternehmen und Forschungseinrichtungen, die nach Handlungsfeldern und Unternehmensgröße differenzieren, werden noch nicht in strukturierte Angebote umgesetzt: Maria Moser-Simmill erarbeitete 2008 in einer Stellungnahme für den Programmbereich FEMtech Karriere einen Maßnahmenkatalog entsprechend der Handlungsfelder und Unternehmensgröße. Auf der Website empfiehlt FEMtech den Unternehmen zwar, nach Unternehmensgröße und vorhandenen Strukturen passende Maßnahmen zu wählen und verweist auf Publikationen zu Good-Practice-Beispielen, bietet jedoch keinen strukturierten Zugang zu den Modellbeispielen an.

Keine explizite Zielgruppe von FEMtech sind Strukturknoten bzw. MultiplikatorInnen im FTI-politischen Bereich, wie z. B. Industriellenvereinigung, Wirtschaftskammern, Branchennetzwerke oder andere Förderagenturen außer FFG, die zur Ausdehnung des Netzwerkes von FEMtech genutzt werden könnten.

\subsection{Strategien}

Zur Erreichung der Ziele bietet FEMtech seit 2007 die drei Programmbereiche „Förderung“, „Aktivitäten“ und „Wissen“ an. ${ }^{12}$ Zentrale Strategie von FEMtech ist die Kombination von dem in der österreichischen FTI-Politik bewährten Ansatz der Programmförderung mit dem neuen Ansatz „Awareness Building“. Die Awareness-Strategie ist ein Alleinstellungsmerkmal des FEMtech Programms.

Der Programmbereich „Förderung“ beinhaltet die drei Programmlinien „FEMtech Karriere“, „FEMtech Karrierewege“ und „FTI-Projekte“. Hierdurch sollen die gesetzten Ziele in den Bereichen „Humanressourcen“ und „Gender in der Forschung"verfolgt werden. Finanzielle Anreize haben eine Signalwirkung für Unternehmen. Aufgrund der unscharfen Differenzierung der Zielgruppen (s. o.) scheinen die Strategien, die mit dieser Programmlinie zur Erreichung von Chancengleichheit führen sollen, noch nicht hinreichend auf die Bedarfe der verschiedenen Zielgruppen ausgerichtet. Die fehlende Bedarfsanalyse und Differenzierung bei den Zielgruppen führt dazu, dass die Strategien der Förderlinien nicht immer zielgruppenorientiert sind. So geht z. B. aus der Beschreibung der Programmlinie „FTI-Projekte“ nicht eindeutig hervor, mit welchen Strategien und Maßnahmen bei WissenschafterInnen und ErfinderInnen in den verschiedenen Disziplinen des FTI-Bereichs Gender-Bewusstsein hergestellt oder verändert werden soll.

Die Inkongruenzen bei den Zielen und Zielgruppen setzen sich in den Programmlinien fort: So sind im Programmbereich Förderung zwar die Unternehmen und Forschungseinrichtungen die primäre Zielgruppe. Indirekt sind viele Strategien jedoch auf Frauen und Nachwuchswissenschafterinnen bzw. Technikerinnen ausgerichtet, was wiederum zu dem Eindruck führt, FEMtech sei ein Frauenförderungsprogramm.

12 Bis 2007 hießen die Programmlinien „FEMtech Karriere“, FEMtech Forschungsunternehmen“ und „FEMtech Fachhochschulen“, zur weiteren Analyse fehlt das Programmdokument von 2004. 
Übergreifend über alle Programmlinien des Bereichs „Förderung“ sollen die Programmbereiche „Aktivitäten“ und „Wissen“ zur Sichtbarmachung der Potenziale von Frauen in Forschung und Technologie beitragen. Dabei soll der Bereich „Aktivitäten“ der „Vernetzung, Bewusstseinsbildung und Sensibilisierung sowie zur Sichtbarmachung von Frauen in Forschung und Technologie“ (vgl. FEMtech (o.J.b)) dienen. Mit dem Programmbereich „Wissen“ sollen zentrale Informationen rund um das Thema Frauen in Forschung und Technologie und Gender Mainstreaming aufbereitet und leicht zugänglich gemacht werden. In der Programmlinie „FTI-Projekte“ sind Ziele und Strategien weder eindeutig formuliert noch kohärent. Auf der Website ist die im Programmdokument beabsichtigte Veränderung bei den Wissenschafterinnen und Wissenschaftern nicht mehr als Ziel aufgeführt. In den Jahresberichten ab 2008 ist die Sensibilisierung von WissenschafterInnen für das Thema „Gender“ hingegen als Ziel neu aufgenommen worden.

Es ist fraglich, ob die Förderung von Forschungsprojekten mit GenderDimension ohne eine begleitende aktive Sensibilisierung der WissenschafterInnen ausreicht, um bei den beteiligten Forscherinnen und Forschern die Akzeptanz und das Interesse für das Thema „Gender“ in der Forschung zu erhöhen. Allein aus der Beteiligung an einem Forschungsprojekt mit GenderDimension folgt nicht automatisch eine grundsätzliche und systematische Sensibilisierung hinsichtlich der Ausrichtung von Forschungsinhalten an den Interessen und Bedürfnissen von Frauen. Hierfür bedarf es flankierender Unterstützungsmaßnahmen, wie z. B. Gender Trainings, wissenschaftsbasierter Wissenstransfer zu den Elementen von Gender in Forschung allgemein und speziell für den Fachbereich. Die Zielgruppe der ForscherInnen und ErfinderInnen ist eine sehr heterogene Gruppe. Um deren Bedarfe zu ermitteln, bedarf es eines engen Austausches mit der Scientific Community. 


\subsection{Logic Chart}

Tabelle 2: Logic Chart

\section{Förderung}

\section{Karrierewege}

\section{Karriere}

Anteil von Frauen in FTI erhö- Anteil von Frauen in FTI erhö- Steigerung der Qualität u. hen, Chancengleichheit fördern
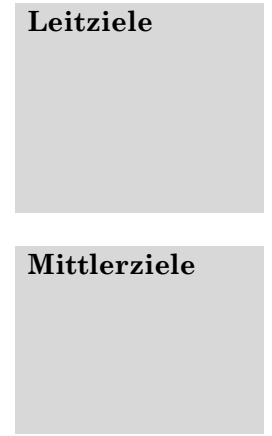

Handlungsziele Verbesserung der Strukturen, Rahmenbedingungen zum Auf-
Rahmenbedingungen, der Situ- bau, Förderung und Begleitung ation u. der Karrierechancen von Studentinnen von Frauen

Attraktivität der Ausbildungen Gewinnung von Nachwuchswisund Berufe in

steigern innen

Akzeptanz und Interesse de beteiligten ForscherInne für das Thema Gender in Forschungsprojekten erhöhen

Technologieintensive Unter- Technologieintensive Unterneh- Technologieintensive Unternehmen, außeruniversitäre men, außeruniversitäre For- nehmen, außeruniversitäre Forschungseinrichtungen Forschungseinrichtungen, EinzelforscherInnen

Lösungen, Akzeptanz von Produkten u. Technologien

Forschungsinhalte auf die Bedürfnisse und Interessen von Frauen ausrichten

Zielgruppen

\begin{abstract}
schungseinrichtungen
\end{abstract}

\section{Aktivitäten}

Wissen

Anteil von Frauen in FTI erhöhen

\section{Bewusstsein für die Themen „Gender“ und „Frauen in} FTI" schaffen

Sichtbarmachung, Vernetzung fördern

Technologieintensive

Unternehmen, außeruniversitäre Forschungseinrich tungen, Frauen in FTI, FTI politische Community
Anteil von Frauen in FTI erhöhen

Wissen zum Thema Frauen in Forschung und Technologie und Gender erhöhen

Zentrale Informationen zum Thema Frauen in Forschung und Technologie und Gender Mainstreaming aufbereiten und leicht zugänglich machen

Technologieintensive Unternehmen, außeruniversitäre Forschungseinrichtungen, Frauen in FTI, FTI politische Community 


\section{Förderung}

\section{Karrierewege}

Maßnahmen zur Verbesserung der Chancengleichheit fördern
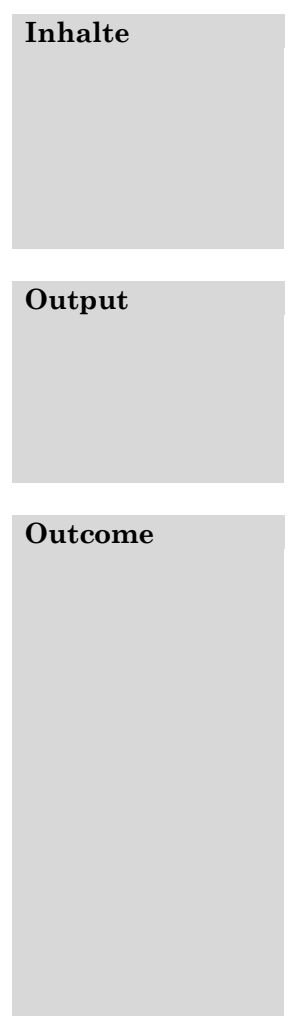

\section{Anzahl der eingereichten} und geförderten Anträge, Bekanntheit und Zufriedenheit

Steigerung des Frauenanteils bei Neueinstellungen, Steigerung des Anteils von Frauen in Führungspositionen, Verbesserung der Rahmenbedingungen für WorkLife-Balance, Verankerung des Gleichstellungsgedankens in der Unternehmenskens in der Unternehmens-
kultur, Erhöhung der Gendersensibilität,

strukturelle/organisatorische Änderungen in der Organi-

sation zur Förderung von

Frauen

Quellenangabe: ARGE „3C“ (basierend auf FEMtech Programmdokument; FEMtech Website)
Kooperation zwischen Hoch- $\quad$ Forschungsprojekte mit chule und Unternehmen stär- Gender-Dimension fördern ken

Programmlinie FEMtech Karrierewege

Anzahl der eingereichten und geförderten Anträge, Bekanntheit und Zufriedenheit

Schaffung von Angeboten zur Vorbereitung von Studentinnen auf Karrieren in F\&E, Qualifizierung von Studentinnen, Erhöhung des Anteils der Absolventinnen, die den Berufseinstieg in $\mathrm{F} \& \mathrm{E}$ suchen
Anzahl der eingereichten und geförderten Anträge, Bekanntheit und Zufriedenheit

teigerung der Bedeutung der Gender-Dimension in Forschungsprojekten Steigerung des GenderBewusstseins der ForscherInnen

\section{Aktivitäten}

Wissen

Öffentlichkeits- u. AwarenessMaßnahmen

Expertinnen-Datenbank, Expertin des Monats, Netzwerk treffen, Forum NaWi)(Tech,

Auszeichnungen, Gend Auszeichnungtail, FEMtech-Website, Ment ring, FEMtech meets

Anzahl der Einträge, Veran staltungen, Teilnehmenden, AbonnentInnen, Bekanntheit des Angebots, Zufriedenheit der Nutzenden, Webseite: Anzahl der Besuche, Downloads

Sichtbarmachung erfolgreicher Forscherinnen und Technikerinnen, Stärkere Diskussion des Themas „Frauen in FTI“ in Osterreich, Verdeutlichen der

Notwendigkeit zur Erschlievon Frauen in F\&E, Förderung von Frauen in F\&E, For

der Vernetzung von FTIder" und Frauen in FTI
Monitoring, Bereitstellung on Daten, Studien u. Publikationen

Daten, FEMtech Publikationen, Download-

Publikationen, Literaturlisten

Anzahl der eigenen Publikationen nach Themen und

Publikationsart

Bekanntheit und Nutzung, Wissens-

/Kenntnisveränderung 


\subsection{Conclusio im internationalen Kontext}

Die von FEMtech ursprünglich gesetzten Ziele und die Ausrichtung auf eine breite, heterogene Zielgruppe basierten auf dem zu Beginn des Programms notwendigen Fokus auf Sichtbarmachung, Sensibilisierung und Bewusstseinsbildung. FEMtech hat die gleichstellungspolitischen Entwicklungen auf dem Gebiet „Frauen in FTI“ seitdem aufgegriffen und seine Ziele flexibel angepasst. Die Schwerpunktsetzung von der Erhöhung des Frauenanteils auf die Veränderung der Strukturen und Arbeitsbedingungen ist sehr positiv zu bewerten. Diese Veränderungen werden jedoch nicht deutlich genug nach außen kommuniziert. Auch sind die Konsequenzen aus der Zielverschiebung für die Konzeption des Programms, für die Zielgruppen und Strategien nicht stark genug berücksichtigt worden. Die Förderung der Chancengleichheit in den Unternehmen findet nicht ausreichend Niederschlag in Strategien.

Die Vielfalt der Zielgruppen ist ein Alleinstellungsmerkmal von FEMtech und dient dazu, eine breite Öffentlichkeit mit dem Thema „Frauen in FTI“ zu erreichen. Für die passgenaue Entwicklung von Strategien bedarf es aber einer genaueren Differenzierung der Zielgruppen und einer zielgruppenspezifischen Bedarfsanalyse. FEMtech hat bezüglich der Zielgruppen einen breiten Ansatz gewählt, der Kern von FTI-Unternehmen, Forschungseinrichtungen und Frauen in Forschung und Technologie blieb dabei gleich. Der Wunsch, alle relevanten Zielgruppen im Sinne eines ganzheitlichen Ansatzes zu erreichen, birgt das Risiko, durch eine mangelnde Fokussierung der Ressourcen die bisher erfolgreiche Arbeit der Sensibilisierung nicht ausreichend fortsetzen zu können.

Im Vergleich mit den internationalen Fallbeispielen hebt sich FEMtech durch die Kombination von Förderlinien und Awareness-Maßnahmen deutlich ab. Ähnlich wie FEMtech hat die Mission pour la place des femmes au CNRS (MPDF) drei wesentliche Ziele: die Stärkung von Gender in der Forschung, die Förderung der Chancengleichheit und die Sensibilisierung zum Thema „Frauen in FTI“. Ein direkter Vergleich mit FEMtech hinsichtlich der Zielgruppen und Strategien ist nicht möglich, da die MPDF insbesondere Veränderungen innerhalb der eigenen Organisation bewirken will (vgl. Kapitel 2.2.3 „Mission pour la place des femmes au CNRS (MPDF)"). Das ABI ist ausschließlich auf die Förderung von Frauen und ihrer Vernetzung ausgerichtet und hat daher hinsichtlich der Zielgruppen und Strategien nur geringe Überschneidungen mit FEMtech. Das UKRC strebt die Gleichstellung von Frauen und Männern im MINT-Bereich an und unterstützt mit einer Bandbreite von Angeboten sowohl Organisationen als auch Frauen mit Beratung und Serviceangeboten. Darüber hinaus versucht das UKRC, Einfluss auf die Gleichstellungspolitiken der Regierung, einflussreicher Behörden und auch innerhalb von Organisationen zu nehmen. Diese Strategie unterscheidet sich aufgrund der Serviceausrichtung und der individuellen Angebote für Organisationen und Frauen wesentlich von der Förderstrategie von FEMtech. Auch beim ABI findet sich der Beratungsansatz z. B. im „Executive Briefing Program“, in dessen Rahmen versucht wird, das Verständnis und das Engagement der Leitungen von Technologie-Unternehmen hinsichtlich der Rekrutierung und Förderung von Frauen zu verbessern. 
Die Zielgruppen werden von ABI und dem UKRC direkter und differenzierter angesprochen. Insbesondere die Webseiten dieser Einrichtungen bieten den Zielgruppen einen gezielteren Zugang als dies bei FEMtech der Fall ist.

Gemeinsam ist allen drei Einrichtungen, dass sie ebenso wie FEMtech auf unterschiedliche Weise die Strategie der Sichtbarmachung von Frauen und der Sensibilisierung und Bewusstseinsbildung für das Thema Frauen in Forschung und Technologie verfolgen. Alle drei Einrichtungen bieten dazu in unterschiedlichem Umfang Datenmaterial und Informationen zu Gleichstellung in Forschung und Technologie an. Das ABI setzt zudem auf Preisverleihungen, um die Beiträge von Frauen im Technologiebereich zu honorieren. Auch das UKRC vergibt einen Preis für „Women of Outstanding Achievement“. Die MPDF im CNRS beteiligt sich an nationalen und internationalen Kolloquien, Fachmessen, Veranstaltungen und Preisverleihungen, die weibliche Vorbilder fördern. Das UKRC und das ABI haben im Gegensatz zu FEMtech einen stärkeren Fokus auf die öffentliche Auszeichnung der Preisträgerinnen und erreichen damit eventuell ein breiteres Publikum. Hinsichtlich der Präsentation der ausgezeichneten Frauen auf der Webseite setzt sich FEMtech allerdings positiv im Verhältnis zu allen Fallbeispielen ab. Die Expertin des Monats ist auf der Startseite prominent platziert.

Im Ergebnis liegt FEMtech mit seinen gleichstellungspolitischen und inhaltlichen Schwerpunkten auf Chancengleichheit und Awareness-Building im internationalen Vergleich im Trend. Der Ansatz der „Chancengleichheit“ findet jedoch nicht ausreichend Niederschlag in den Strategien und wird nicht deutlich genug nach außen kommuniziert. Das Handlungsfeld (Awareness, Chancengleichheit und Gender in der Forschung) ist zu breit. Die ReferenzEinrichtungen ABI und UKRC konzentrieren sich auf ein primäres Ziel. Auch hinsichtlich der Zielgruppen bzw. Multiplikatoren sind die vorgenannten Einrichtungen fokussierter und verfügen über ein differenzierteres und spezifischeres Angebot. 


\section{FEMtech Portfolio-Analyse}

Die Analyse des FEMtech Portfolios basiert auf folgenden Quellen:

1. Dokumentenanalysen der veröffentlichten FEMtech Produkte, Programmdokumente, Jahresberichte etc.

2. Auswertung und Analyse von Primärdaten zur Verbreitung und Nutzung des FEMtech Portfolios (Anmelde-/Teilnahmelisten, Webstatistiken, Medien-Clippings, Datenbank-Dumps etc.)

3. flächendeckende Online-Befragung (Vollerhebung) von FEMtech Adressatinnen $(\mathrm{n}=352)$ und Fördernehmenden $(\mathrm{n}=37)$ sowie - als Vergleichsgruppe - generell von Projektleitungen aus FTI-Unternehmen und -Organisationen, die seit 2004 FFG-Forschungsförderung beantragt haben ( $\mathrm{n}=1.583)$;

4. Fokusinterviews mit FEMtech Förderungsnehmerinnen

5. qualitative Interviews mit VertreterInnen des oberen Managements aus FTI-Unternehmen/-Forschungseinrichtungen, FTI-politischen AkteurInnen sowie aktuellen und ehemaligen Programm-UmsetzerInnen.

6. Self-Assessment der Programm-UmsetzerInnen

7. Auswertung der FFG-Förderungsdatenbank: a) FEMtech Förderungen 2004 bis Oktober 2010 und als Vergleichsgruppe der Fördernehmenden $(\mathrm{N}=130)$ b) Unternehmensstruktur aller FFG-FördernehmerInnen 2004 bis Oktober $2010(\mathrm{~N}=$ ca. 9.500)

Dabei bilden Primärdaten die grundlegende Basis für die Interpretation der tatsächlichen Nutzung und Zielgruppenerreichung der FEMtech Maßnahmen. Ergebnisse aus der Online-Befragung und qualitative Interviews werden zur Vertiefung und interpretatorischen Einordnung hinzugezogen und liefern darüber hinaus Erkenntnisse zur Bekanntheit und Zufriedenheit mit den Instrumenten.

\subsection{FEMtech Förderungen}

FEMtech bietet im Bereich „Förderungen“ drei Förderlinien für unterschiedliche Problemlagen der Ungleichheit im Zugang von Frauen zu Forschung und Technologie an. Dies ist zum einen die Förderlinie „Karriere“, die seit Beginn von FEMtech bereitgestellt wird, 2008 kamen „Karrierewege“ und „FTIProjekte“ hinzu. Unterstützen die beiden Ersteren Forscherinnen und Technikerinnen in Unternehmen sowie Studentinnen an den naturwissenschaftlich/technischen Universitäten im Übergang zur Industrie, so will die dritte Förderlinie die Akzeptanz von Genderinhalten in den Forschungs- und Entwicklungsaktivitäten steigern. Die drei Förderlinien werden von der FFG betreut, in der Akquisition und Förderberatung waren 2008-2010 ebenso die ÖGUT und D\&Z Consulting involviert.

Datengrundlage für die Analyse des Förderportfolios sind die Programmdokumente, die Programmausschreibungen sowie die Förderunterlagen der FFG. Weiters wurden bereits vorhandene Zusammenstellungen (z. B. Jahresberichte, Broschüren) in die Analyse miteinbezogen. Als Datenbasis für die Förderauswertungen wurden Daten von der FFG zur Verfügung gestellt. Diese be- 
inhalten alle Projekteinreichungen der drei Förderlinien ab 2004. Für die Förderlinie Karriere wurden die Datenblätter der einreichenden Unternehmen soweit vorhanden - ausgewertet. Hier wurden zudem Daten für Förderungsaktivitäten vor 2007 zur Verfügung gestellt. Die FEMtech Mitarbeiterinnen der FFG ergänzten in Formularen die inhaltlichen Schwerpunkte der Projekte nach Kategorien; für FEMtech Karriere wurde dazu auch eine frühere Erhebung von Joanneum Research als Basis zur Verfügung gestellt. Zusätzlich wurde von der FFG eine Auswertung der Organisationsstruktur aller FFGFördernehmenden seit 2004 übermittelt, um einen Vergleich zur Gruppe der FEMtech Fördernehmenden erstellen zu können.

In den Programmgesprächen und im Self-Assessment mit den ProgrammUmsetzerInnen wurden relevante Aspekte zur Förderabwicklung erfasst. Beide Onlinebefragungen (FFG-Fördernehmende und FEMtech NutzerInnen ) ermittelten wichtige Erkenntnisse zur Nutzung und geben Verbesserungsvorschläge der einreichenden Zielgruppe, ergänzt wurden diese durch Fokusinterviews mit unterstützten Frauen im Rahmen der FEMtech Förderlinien sowie mit ehemaligen FEMtech Programmleiterinnen.

\subsubsection{FEMtech Karriere}

FEMtech Karriere war die erste Förderlinie und wurde bereits mit Programmbeginn gestartet. Geplant war, ein leicht zugängliches Impulsprogramm aufzulegen, indem jederzeit Anträge eingereicht werden, die in wenigen Wochen entschieden sind und so rasch Maßnahmen in FTI-Unternehmen und Forschungseinrichtungen umgesetzt werden können. „Small \& quick“ umschreibt am besten den Zugang. Das zu Beginn mit 25.0000 € Bundesförderung (ab 2008: $50.000 €$ ) pro Projekt sehr klein dimensionierte FFG-Programm befand sich auch aufgrund seiner inhaltlichen Gestaltung in einem "Sonderbereich“: Die Inhalte des Antrages beziehen sich nicht auf Forschung, technologische Entwicklungen oder Innovationsvorhaben, sondern auf überwiegend strukturelle Maßnahmen zur Frauenförderung und Chancengleichheit in FTIUnternehmen und außeruniversitären Forschungseinrichtungen. 
Tabelle 3: Die wesentlichen Eckpunkte von FEMtech Karriere:

\begin{tabular}{|c|c|}
\hline FEMtech Karriere & Kurzbeschreibung \\
\hline Ziel & $\begin{array}{l}\text { Strukturelle Verbesserungen in FTI-Unternehmen und außeruniversitä- } \\
\text { ren Forschungseinrichtungen zur Steigerung des Anteils von Frauen in } \\
\text { Forschung und Technologie }\end{array}$ \\
\hline Zielgruppen & $\begin{array}{l}\text { - forschungs- und/oder technologieintensive Unternehmen } \\
\text { - außeruniversitäre Forschungseinrichtungen mit naturwissenschaft- } \\
\text { lich-technischem Schwerpunkt }\end{array}$ \\
\hline Laufzeit & mindestens 6 Monate und maximal 2 Jahre \\
\hline Einreichung & Antragsverfahren; laufende Einreichung möglich \\
\hline Förderungsgrundlage & De minimis \\
\hline Förderungshöhe & $\begin{array}{l}\text { - } \text { maximal } 70 \% \text { der förderbaren Gesamtkosten } \\
\text { - maximal } 50.000 € \text { Förderung (seit Mitte 2008, vorher } 25.000 € \text { ) }\end{array}$ \\
\hline Programmumsetzung & $\begin{array}{l}\text { - } \text { Einreichberatung und Programmbetreuung: FFG } \\
\text { - Akquisition und Förderberatung: D\&Z Consulting }\end{array}$ \\
\hline
\end{tabular}

Quellenangabe: FEMtech Programmdokument; Interviews mit Programm-UmsetzerInnen

Seit dem Start der Förderlinie FEMtech Karriere im Jahr 2004 kann ein stetiger Anstieg der geförderten Projekte (2010 nur bis Oktober erfasst) festgestellt werden.

Abbildung 7: Anzahl der geförderten FEMtech Karriere-Projekte im Zeitverlauf

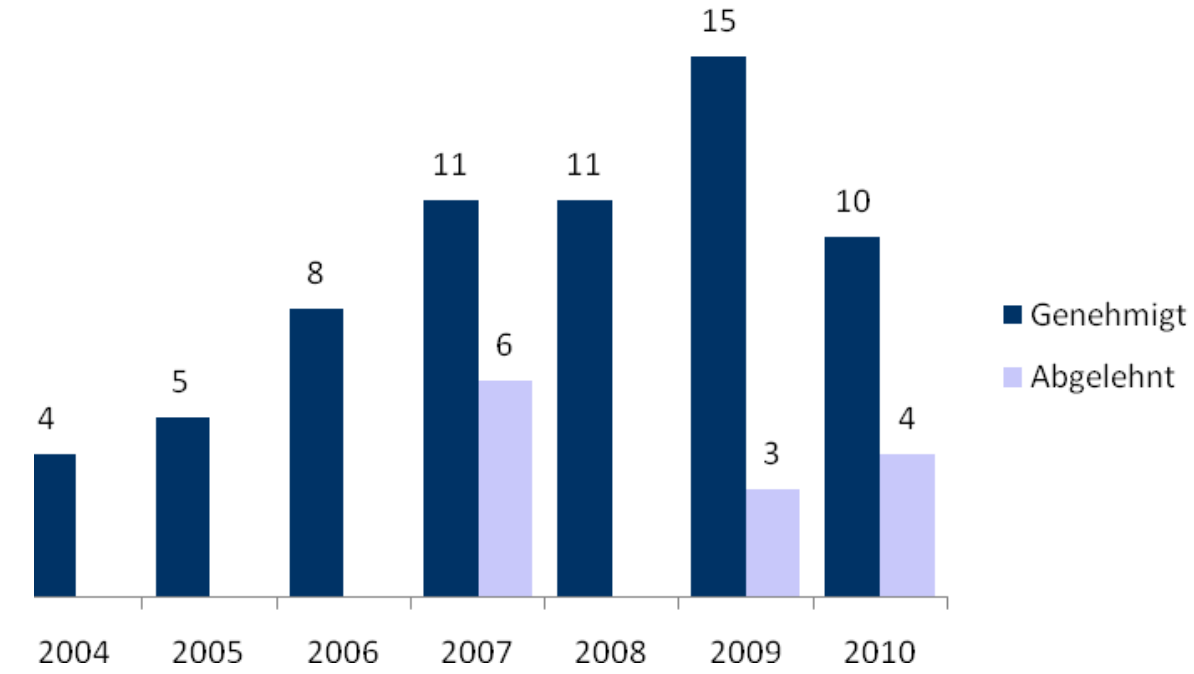

Quellenangabe: FFG-Förderdatenbank (2004 - Okt. 2010), N = 77

Insgesamt wurden in den Jahren 2004 bis Oktober 201077 Anträge eingereicht und 64 FEMtech Karriere-Projekte gefördert. Hiezu muss eine Anmer- 
kung bezüglich der Daten erfolgen: Zu Beginn wurden FEMtech KarriereProjekte, insbesondere im Bereich der außeruniversitären Forschung, auch mit anderen Instrumenten unterstützt, jedoch unter FEMtech Karriere geführt dadurch ergibt sich auf der FEMtech Website und in Broschüren eine andere Gesamtsumme.

Tabelle 4: Förderquote FEMtech Karriere-Projekte

\begin{tabular}{l|l} 
Karriere-Projekte & Anzahl \\
\hline Eingereicht & 77 \\
\hline Gefördert & \\
FFG-Förderdatenbank & 64 \\
(Projektliste FEMtech Karriere) & $(71)$ \\
\hline Abgelehnt & 13 \\
\hline Förderquote & $83 \%$
\end{tabular}

Quellenangabe: FFG-Förderdatenbank (2004 - Okt. 2010), FEMtech Projektliste

Da dem Evaluationsteam als Auswertungsbasis die FFG-Förderdaten mit der entsprechenden Zuordnung aus den Fördertiteln - nämlich FEMtech Karriere - zur Verfügung standen, beziehen sich die folgenden Auswertungen auf diese Daten. Die sieben nicht erfassten Projekte betreffen Einrichtungen der außeruniversitären Forschung und ein Kompetenzzentrum, alle im Zeitraum 2004 bis 2006. Abgelehnt wurden überwiegend Anträge von Unternehmen (12 von 14 Ablehnungen insgesamt). Dabei wurden insbesondere zu Beginn der FEMtech Karriereförderung kaum Projekte abgelehnt. Dies ist zum einen dadurch begründet, dass die ersten einreichenden Unternehmen bereits themensensibilisiert waren und zum anderen bereits von Beginn an eine intensive Beratung der Einreichenden angeboten wurde - sowohl von der FFG als auch von D\&Z Consulting, die mit der Akquisition und Beratung von FTI-Unternehmen bezüglich FEMtech Karriere beauftragt waren.

Tabelle 5: Gesamtkosten und Fördervolumina der genehmigten FEMtech Karriere-Projekte

\begin{tabular}{l|c|c|c|c|c|c|c|c} 
& 2004 & 2005 & 2006 & 2007 & $\left.2008^{*}\right)$ & 2009 & 2010 & Gesamt \\
\hline $\begin{array}{l}\text { Gesamtkosten lt. } \\
\text { Beschluss [€] }\end{array}$ & 215.444 & 344.622 & 325.935 & 442.370 & 583.529 & 971.503 & 793.253 & $\mathbf{3 . 6 7 6 . 6 5 6}$ \\
\hline Bundesförderung [€] & 100.000 & 124.500 & 179.225 & 267.355 & 323.974 & 562.501 & 432.085 & $\mathbf{1 . 9 8 9 . 6 4 0}$
\end{tabular}

*) ab Mitte 2008 wurde die maximale Förderungshöhe von $25.000 €$ auf $50.000 €$ verdoppelt.

Quellenangabe: FFG-Förderdatenbank (2004 - Okt. 2010)

Insgesamt wurden mit einer Bundesförderung von ca. 2 Mio. $€$ in sieben Jahren Maßnahmen zu Frauenförderung und Chancengleichheit im Bereich von 3,68 Mio. € getätigt. FEMtech Karriere-Projekte wurden von folgenden Organisationstypen durchgeführt (die außeruniversitären Forschungseinrichtungen der Jahre 2004 bis 2006 sind nicht berücksichtigt): 
Abbildung 8: Geförderte FEMtech Karriere-Projekte nach Organisationstyp

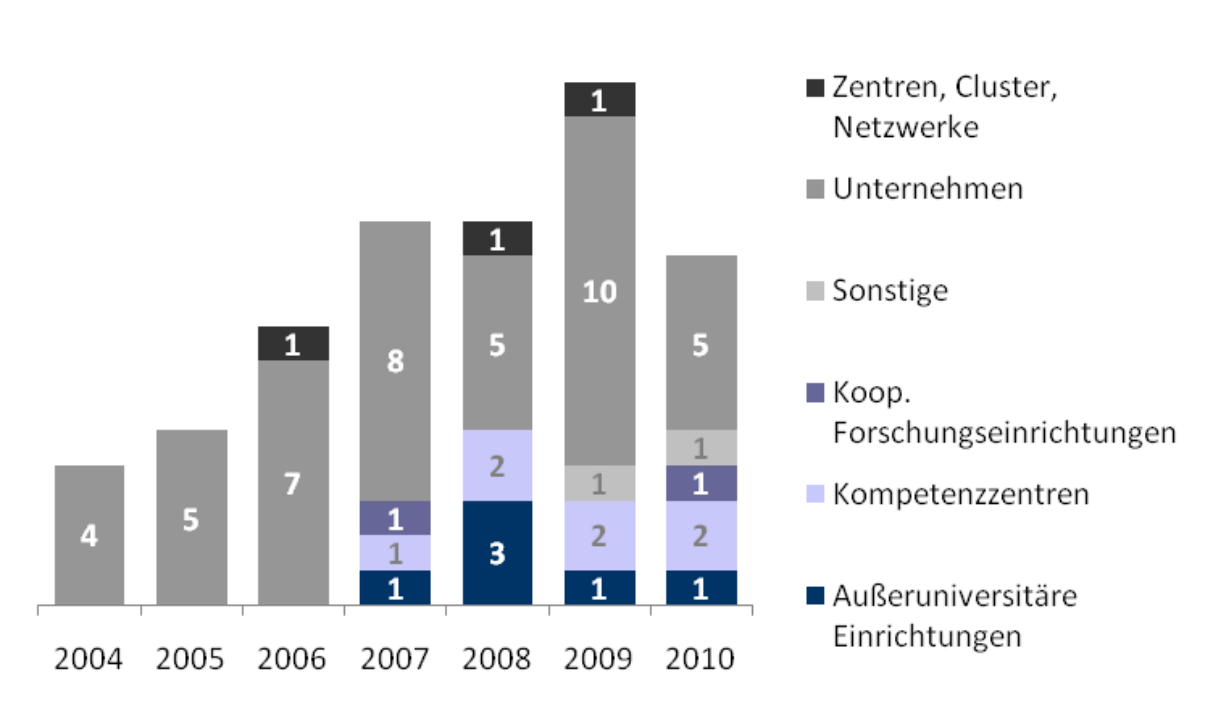

Quellenangabe: FFG-Förderdatenbank (2004 - Okt. 2010), N = 64

Die Förderlinie FEMtech Karriere sprach zu Beginn insbesondere größere Unternehmen an, die bereits im Bereich Chancengleichheit und Frauenförderung sensibilisiert waren und hier gezielt Maßnahmen setzen wollten. Die großen außeruniversitären Forschungseinrichtungen (AIT, Joanneum Research Forschungsgesellschaft etc.) begannen ebenso bereits 2004 mit Projekten (diese sind in diesem Datensatz jedoch nicht abgebildet). Erst nach zwei- bis dreijähriger Laufzeit erkannten andere FTI-Einrichtungen, vor allem Kompetenzzentren, die Möglichkeit, gezielt frauenfördernde Maßnahmen zu setzen. Das Segment der außeruniversitären Forschungseinrichtungen und FTIEinrichtungen macht seit ca. drei Jahren ca. die Hälfte der FEMtech KarriereProjektdurchführenden aus. Eine Einteilung der Unternehmen nach Größenordnung zeigt (wobei ein Viertel unbekannt ist): $39 \%$ sind Großunternehmen, $16 \%$ mittlere Unternehmen und $20 \%$ kleine Unternehmen.

Abbildung 9: Geförderte FEMtech Karriere-Projekte nach Bundesland

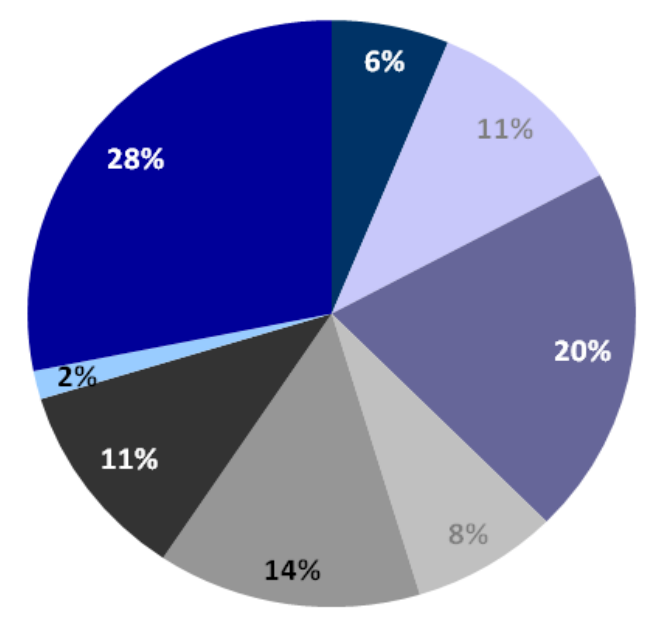

Niederoesterreich

- Oberoesterreich

जalzburg

n Steiermark

- Tirol

- Vorarlberg

- Wien

Quellenangabe: FFG-Förderdatenbank (2004 - Okt. 2010) 
Aus Wien und Oberösterreich kommen beinahe 50 \% der FEMtech KarriereProjekte, Vorarlberg ist kaum vertreten, gering auch Kärnten und Salzburg. Aus dem Burgenland gibt es kein einziges FEMtech Karriere-Projekt. Da FEMtech Karriere beinahe zur Hälfte von FTI-Einrichtungen (Netzwerke, Kompetenzzentren, außeruniversitäre Forschungseinrichtungen) genutzt wird, spiegelt sich hier deren regionale Verteilung in Österreich sehr gut wider und begründet damit auch die geringe Beteiligung dieser Bundesländer. Einzig die Steiermark wäre hier aufgrund ihres Potenzials an FTI-Einrichtungen stärker einzuschätzen (vgl. Statistik Austria 2010, S.157).

Abbildung 10: Inhaltliche Schwerpunkte FEMtech Karriere-Projekte

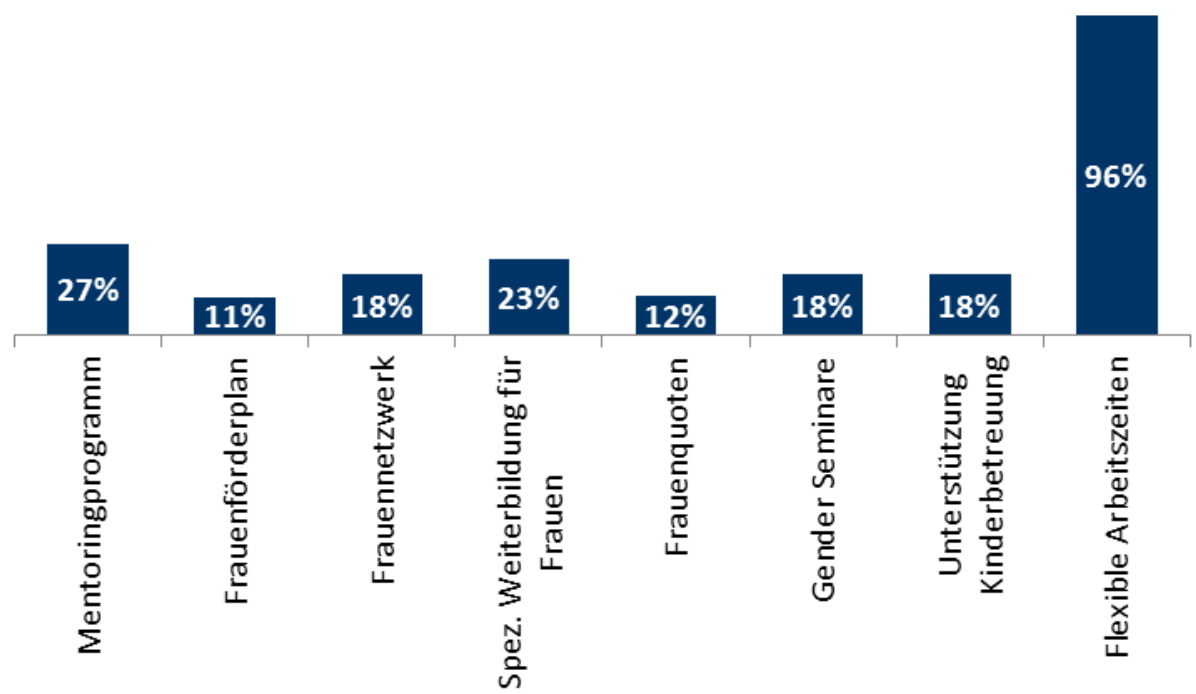

Quellenangabe: FFG/FEMtech Programmmanagement, Joanneum Research, Datenbasis: FEMtech Karriere-Broschüre

Flexible Arbeitszeiten ist bei beinahe allen FEMtech Karriere-Projekten ein Projektinhalt, kein weiteres Thema erreicht diese Präsenz. Mentoring und Frauenförderpläne sowie „Frauenquoten“ sind zumeist in Großunternehmen Projektinhalt. Spezifische Weiterbildungen für Frauen bieten vor allem Kleinunternehmen im Rahmen von FEMtech Karriere an. Gender-Seminare werden sowohl von Klein- als auch Großunternehmen durchgeführt.

Von Anfang an war den Programm-UmsetzerInnen bewusst, dass es schwierig sein wird, FTI-Unternehmen für Frauenförderung und Chancengleichheit im Unternehmen zu gewinnen - daher wurde die Unternehmensberatung D\&Z Consulting beauftragt, aktiv FTI-intensive Unternehmen zu akquirieren und über FEMtech Karriere und seine Fördermöglichkeiten zu informieren sowie bei der Antragstellung behilflich zu sein. 
Tabelle 6: Akquisitionsaufwand und -erfolg FEMtech Karriere 2008-2010

\begin{tabular}{|c|c|c|c|c|c|}
\hline Unternehmen & $\begin{array}{c}\text { gesamt } \\
\text { (Mailing, } \\
\text { Telefonate) }\end{array}$ & $\begin{array}{c}\text { Erst- } \\
\text { gespräche }\end{array}$ & eingereicht & abgelehnt & genehmigt \\
\hline Wien & 385 & 30 & 9 & 1 & 8 \\
\hline NÖ. & 189 & 10 & 4 & & 4 \\
\hline Stmk. & 236 & 10 & 6 & 2 & 4 \\
\hline Bgld. & 76 & 2 & & & \\
\hline OÖ. & 504 & 9 & 4 & 1 & 3 \\
\hline Knt. & 115 & 2 & 2 & & 2 \\
\hline Sbg. & 130 & 11 & 3 & & 3 \\
\hline Tirol & 209 & 9 & 4 & 1 & 3 \\
\hline \multirow[t]{2}{*}{ Vbg. } & 76 & 1 & 2 & 1 & 1 \\
\hline & 1920 & 84 & 34 & 6 & 28 \\
\hline
\end{tabular}

Quellenangabe: Zusammenstellung D\&Z-Monitoring (2008 bis Okt. 2010)

Die Tabelle zeigt die Schwierigkeit, Unternehmen für die Entwicklung und Umsetzung entsprechender Maßnahmen zu gewinnen. Auf Basis der zahlreichen Informationen, welche durch Mailings und Telefonate zur Verfügung gestellt wurden, konnten in $5 \%$ dieser Unternehmen Erstgespräche vereinbart werden. FTI-Unternehmen sind sehr schwer für chancengleichheitsorientierte Maßnahmen zu motivieren - trotz Förderung. Die „Krisenjahre“ 2008-2009 haben die Aufmerksamkeit der Unternehmen zudem auf andere Themen gelenkt. Lediglich einem Drittel der persönlich in einem Erstgespräch begleiteten Unternehmen ist es gelungen, einen erfolgreichen Antrag einzubringen. Die Einschätzung der Programm-UmsetzerInnen ist diesbezüglich eindeutig: Gäbe es keine Aufmerksamkeit in Richtung gezielte Akquisitionstätigkeit, dann würde es kaum FEMtech Karriere-Projekte in FTI-Unternehmen geben.

\subsubsection{FEMtech Karrierewege}

Laut FEMtech Programmdokument soll FEMtech Karrierewege Kooperationen von Universitäten und Fachhochschulen mit FTI-Unternehmen fördern, um ausgewählten Nachwuchswissenschafterinnen ein Begleit- und Qualifizierungsprogramm zu bieten, damit diese vermehrt ihren Berufseinstieg in F\&Eintensiven Unternehmen finden. 
Tabelle 7: Die wesentlichen Eckpunkte von FEMtech Karrierewege

\begin{tabular}{|c|c|}
\hline FEMtech Karrierewege & Kurzbeschreibung \\
\hline Ziele & $\begin{array}{l}\text { Gewinnung von Nachwuchswissenschafterinnen für F\&E-intensive } \\
\text { Unternehmen durch Vorbereitung von Studentinnen für diesen Kar- } \\
\text { riereweg }\end{array}$ \\
\hline Zielgruppen & $\begin{array}{l}\text { - Tertiäre Ausbildungseinrichtungen mit naturwissenschaftlich- } \\
\text { technischem Schwerpunkt (Universitäten, Fachhochschulen) und } \\
\text { deren Studentinnen } \\
\text { - Forschungs- und/oder technologieintensive Unternehmen }\end{array}$ \\
\hline Laufzeit & mindestens ein Jahr und maximal zwei Jahre \\
\hline Einreichung & $\begin{array}{l}\text { Call-Verfahren, Deutsch sprechende internationale Jury: Der Antrag } \\
\text { ist von Universität bzw. Fachhochschule (Förderungswerberin) in } \\
\text { Kooperation mit mindestens zwei UnternehmenspartnerInnen einzu- } \\
\text { reichen. }\end{array}$ \\
\hline Förderungsgrundlage & FTE-Richtlinie \\
\hline Förderungshöhe & $\begin{array}{l}\text { - Pro Projekt und Antragskonsortium werden maximal } 400.000 € \\
\text { gefördert } \\
\text { - Bildungs- und Ausbildungseinrichtungen max. } 100 \text { \% Förderung } \\
\text { - Unternehmen nach Unternehmensgröße } \\
\text { - } \operatorname{max.} 70 \% \text { Kleinunternehmen } \\
\text { - } \operatorname{max.} 60 \% \text { mittlere Unternehmen } \\
\text { - } \operatorname{max.} 50 \% \text { Großunternehmen }\end{array}$ \\
\hline Programmumsetzung & $\begin{array}{l}\text { - Einreichberatung und Programmbetreuung: FFG } \\
\text { - Förderberatung: ÖGUT }\end{array}$ \\
\hline
\end{tabular}

Quellenangabe: FEMtech Programmdokument; Interviews mit Programm-UmsetzerInnen

FEMtech Karrierewege wird im Wettbewerbsprinzip von der FFG ausgeschrieben, wobei insgesamt zwei Calls in den Jahren 2008 und 2009 mit folgender Projektanzahl stattfanden:

Abbildung 11: Übersicht genehmigter und nicht genehmigter FEMtech Karrierewege-Projekte

4

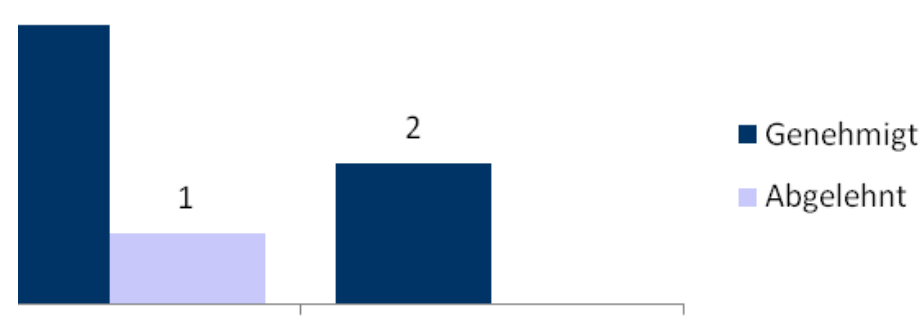

1. Call

2. Call

Quellenangabe: FFG-Förderdatenbank (2004 bis Okt. 2010) 
Insgesamt bedeutet dies für die Förderlinie FEMtech Karrierewege:

Tabelle 8: Förderquote FEMtech Karrierewege-Projekte

\begin{tabular}{l|l} 
Karrierewege-Projekte & Anzahl \\
\hline Eingereicht & 7 \\
\hline Gefördert & 6 \\
\hline Abgelehnt & 1 \\
\hline Förderquote & $86 \%$
\end{tabular}

Quellenangabe: FFG-Förderdatenbank (2004 bis Okt. 2010)

Träger der geförderten FEMtech Karrierewege-Projekte sind:

- Universität für Bodenkultur, Wien

- Technische Universität Wien

- Johannes-Kepler-Universität Linz (2 x)

- Technische Universität Graz

- Fachhochschule Kärnten

Die Trägerschaften bilden auf universitärer Ebene die Landschaft der Technischen Universitäten bzw. Universitäten mit technisch-formal-/ naturwissenschaftlichen Fakultäten in Österreich ab. Im Sektor der Fachhochschulen gäbe es hier mehr naturwissenschaftlich-technische Angebote, die sich jedoch nicht in FEMtech Karriere-Projekten widerspiegeln.

Tabelle 9: Gesamtkosten und Fördervolumina der genehmigten FEMtech Karrierewege-Projekte

\begin{tabular}{l|l|l|l} 
& $\begin{array}{l}\text { Gesamt } \\
\text { 1. Call }\end{array}$ & $\begin{array}{l}\text { Gesamt } \\
\text { 2. Call }\end{array}$ & $\begin{array}{l}\text { Karrierewege } \\
\text { gesamt }\end{array}$ \\
\hline Gesamtkosten lt. Beschluss & $1.885 .192 €$ & $1.317 .549 €$ & $3.202 .741 €$ \\
\hline Bundesförderung & $1.295 .815 €$ & $789.699 €$ & $2.085 .514 €$
\end{tabular}

Quellenangabe: FFG-Förderdatenbank (2004 bis Okt. 2010)

Pro FEMtech Karrierewege-Projekt standen max. $400.000 €$ Bundesförderung zur Verfügung. Es zeigt sich, dass die maximale Förderungshöhe bei drei Projekten beinahe zur Gänze ausgeschöpft wird, bei den anderen liegt sie bei ca. zwei Drittel. Insgesamt wurden für die sechs FEMtech Karrierewege-Projekte 2,085 Mio. € Bundesförderung eingesetzt.

Die Projekte beinhalten eine Kooperation einer Universität oder Fachhochschule mit mindestens zwei Unternehmenspartnern. Träger und Konsortialführer der sechs FEMtech Karrierewege-Projekte sind fünfmal eine Universität und einmal eine Fachhochschule. Insgesamt sieht die Verteilung aller beteiligten Einrichtungen und Organisationen folgendermaßen aus: 
Abbildung 12: Übersicht Organisationstyp Konsortialpartner bei genehmigten FEMtech Karrierewege-Projekten

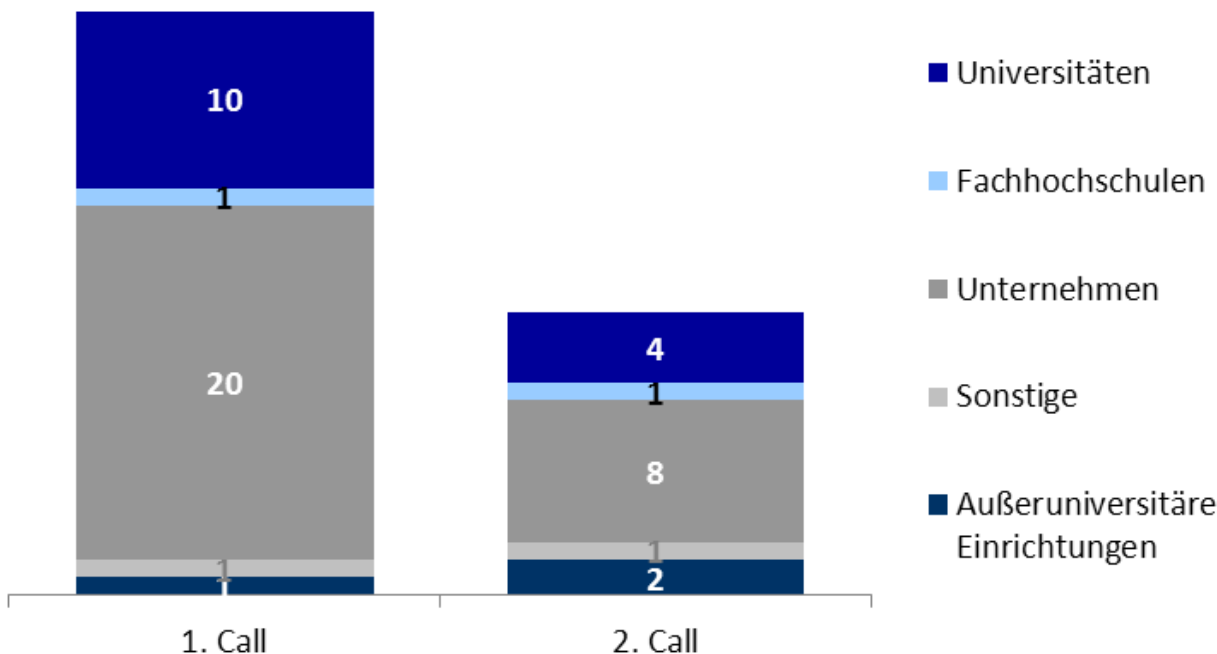

Quellenangabe: FFG-Förderdatenbank (2004 bis Okt. 2010)

$57 \%$ der KooperationspartnerInnen sind Unternehmen, überwiegend Großunternehmen, $33 \%$ Universitäten und Fachhochschulen. Außeruniversitäre Forschungseinrichtungen beteiligten sich nur gering an dieser FEMtech Förderlinie. Die Projektpartnerschaften sind sehr unterschiedlich aufgesetzt: In einigen kooperieren mehrere Universitäten miteinander, andere haben eine Universität und ,ihre“ Unternehmen (im Sinne, dass diese Unternehmen traditionell Abnehmer von AbsolventInnen dieser Universität sind) als Verbund. So gibt es FEMtech Karrierewege-Projekte mit drei Projektpartnern als Mindestanforderung bis zu größeren Konsortien von 13 Partnern.

Abbildung 13: Bundesländerverteilung aller beteiligten FEMtech Karrierewege-Projektpartner

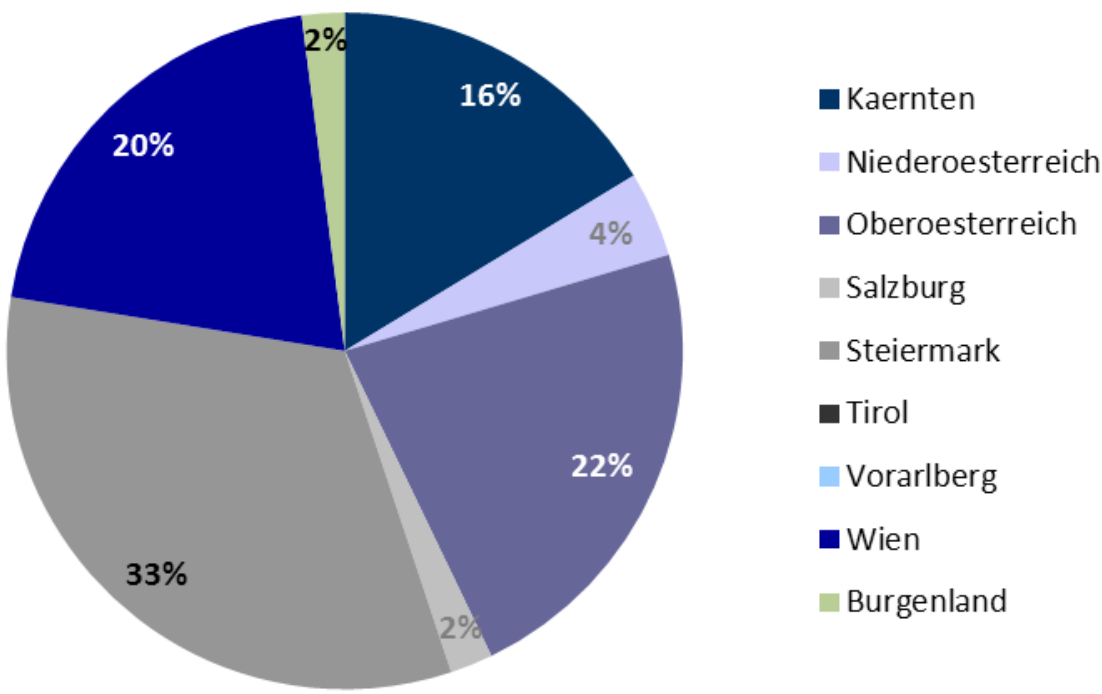

Quellenangabe: FFG-Förderdatenbank (2004 - Okt. 2010)

Mit Blick auf die regionale Verteilung fällt auf: FEMtech Karrierewege gelingt es, den einzigen burgenländischen Projektpartner in einer FEMtech Förderli- 
nie zu integrieren und ist somit im Osten, Süden und der Mitte Österreichs präsent. Jedoch: Der Westen Österreichs fehlt zur Gänze, d. h. in Tirol und Vorarlberg gibt es keinen einzigen Projektpartner. Vor allem Tirol hätte mit Innsbruck als Universitätsstandort hier noch Potenzial. 22 \% der Projektpartner stammen aus Oberösterreich, begründet dadurch, dass die JohannesKepler-Universität Linz Träger von zwei FEMtech Karriereweg-Projekten mit entsprechend großen Projektpartnerschaften ist. In Wien gibt es ebenso zwei Projekte: Die Technische Universität Wien und die Universität für Bodenkultur: Beide haben eher kleine Projektpartnerschaften, daher sind sie in der regionalen Verteilung lediglich an dritter Stelle. Die Technische Universität Graz ist Träger eines FEMtech Karriereweg-Projektes. Die starke regionale Präsenz von einem Drittel der Projektpartner ergibt sich vor allem daraus, dass einige steirische Unternehmen und Forschungseinrichtungen auch in anderen Projektpartnerschaften vertreten sind. Die Fachhochschule Kärnten punktet mit einer großen Projektpartnerschaft mit Kärntner Unternehmen, daher 16 \%.

An Inhalten bieten die sechs FEMtech Karrierewege-Projekte folgende Schwerpunkte für die Teilnehmerinnen an:

Abbildung 14: Inhalte der FEMtech Karrierewege-Projekte

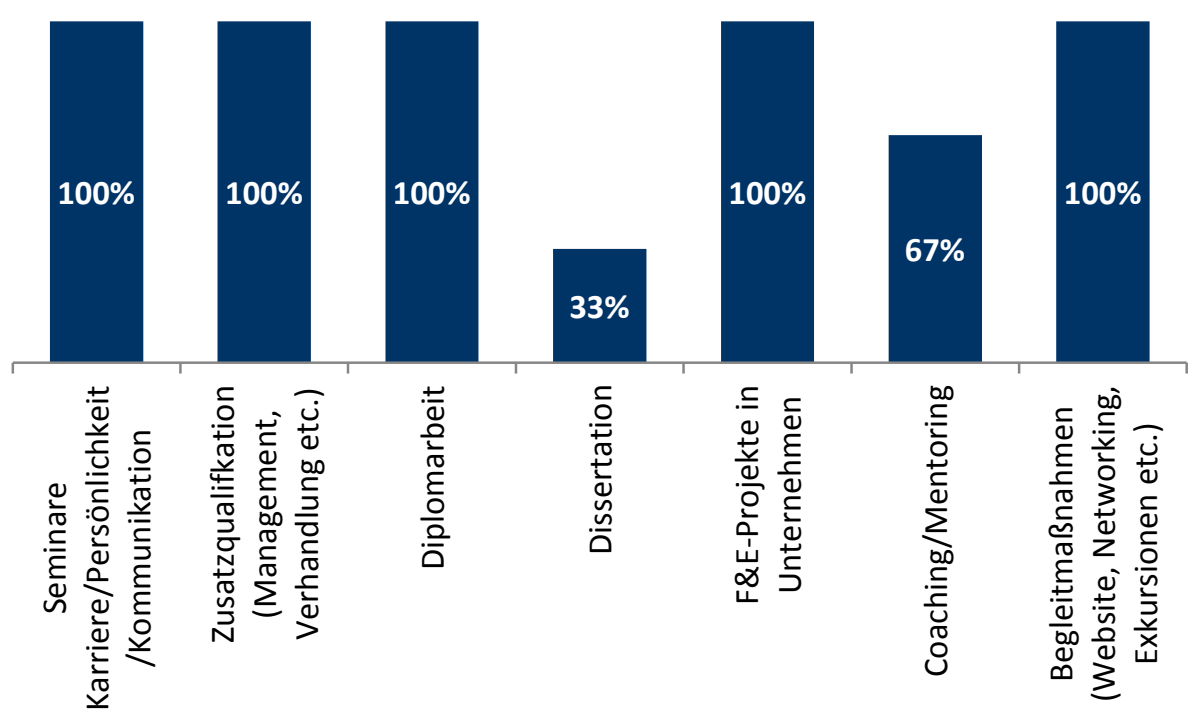

Quellenangabe: FFG/FEMtech Programmumsetzung

Der Schwerpunkt der inhaltlichen Gestaltung liegt auf der Bereitstellung von Praktika und der Ermöglichung von Diplom- und Masterarbeiten, vereinzelt auch von Bachelorarbeiten, Off-the-job-Aktivitäten und Dissertationen in $\mathrm{Zu}$ sammenarbeit mit den Unternehmen. Im Jänner 2010 fand eine 4-tägige „Winterschool" für alle Studentinnen, die an einem Karrierewege-Projekt teilnehmen, statt. 29 Studentinnen nahmen daran teil und erhielten Informationen über Karrierewege in der Industrie, Nutzung von Förderungen sowie Netzwerken.

Die Förderlinie FEMtech Karrierewege bietet ein Begleit- und Qualifizierungsprogramm für Nachwuchswissenschafterinnen, um ihnen dadurch einen Berufseinstieg in F\&E-intensive Unternehmen zu erleichtern. Eine Konsortialpartnerschaft begleitet dabei zwischen 20 und 30 Studentinnen auf ihrem Weg. Insgesamt konnten mit den Maßnahmen aus den Projekten der Förderlinie 
FEMtech Karrierewege bisher 140 Studentinnen (63 Praktika, 4 Bachelorarbeiten, 61 Diplom- und Masterarbeiten, 5 Dissertationen, 7 Off-the-jobAktivitäten) in Österreich erreicht werden. Pro Studentin entfällt dabei je nach Projekt eine Bundesförderung zwischen 8.000 und $20.000 €$ (bzw. $15.000 € \mathrm{im}$ Durchschnitt). Da die Beschäftigungsverhältnisse und Begleitmaßnahmen stark divergieren, ist eine Einschätzungen zu diesem Kostenaufwand sehr schwierig, aber erscheint durch den Vergleich mit den FWFPersonalkostensätzen, welche keine Begleitmaßnahmen beinhalten, nachvollziehbar.

Tabelle 10: Auswahl FWF-Personalkostensätze mit Relevanz zu Beschäftigungsverhältnissen in FEMtech Karrierewegeprojekten

\begin{tabular}{l|c|c} 
& $\begin{array}{c}\text { Personal- } \\
\text { kostensatz } \\
\text { Jahr }\end{array}$ & $\begin{array}{c}\text { Bruttogehalt } \\
\text { Monat }\end{array}$ \\
\hline DoktorandIn & $33.620 €$ & $1.877 €$ \\
\hline Technische Fachkraft, LaborantIn & $28.980 €$ & $1.617,30 €$ \\
\hline Forschungsbeihilfe für DiplomandInnen & $5.280 €$ & $440 €$ \\
\hline Geringfügig Beschäftigte & $6.490 €$ & $374,02 €$
\end{tabular}

Quellenangabe: FWF-Personalkostensätze 2011

In der Art der geförderten Anstellungen der Studentinnen zeigt sich jedoch eine Inkonsistenz im Zusammenhang mit dem Programmdokument. Es wurden hier, neben Dissertationen und Diplomarbeiten, vorrangig Praktika gefördert, zum Teil auch Bachelorarbeiten sowie Off-the-job-Aktivitäten, welche nicht als förderbare Vorhaben im Dokument angeführt sind (vgl. FEMtech 2007, S. 16). Außerdem ist ebendort der Anteil an Praktikumsstellen auf max. $30 \%$ der Gesamtstellen begrenzt. Insgesamt wurden jedoch $45 \%$ der Plätze an Praktikantinnen vergeben (je nach Projekt waren es zwischen $30 \%$ und $57 \%$ ) und weitere 11 Stellen (ca. 8\%) an Bachelorarbeiten und Off-the-jobAktivitäten. Es zeigt sich also, dass mehr als $50 \%$ der Plätze an Maßnahmen vergeben wurden, welche nicht im primären Fokus der förderbaren Maßnahmen (laut Programmdokument) liegen.

\subsubsection{FEMtech FTI-Projekte}

Die Förderlinie FEMtech FTI-Projekte soll Interesse und Akzeptanz für das Thema „Gender“ in Forschungs- und Entwicklungsprojekten sowie technischen Machbarkeitsstudien schaffen und spricht dabei insbesondere die WissenschafterInnen an. Erwartet wird dadurch eine Steigerung der Qualität, bessere Lösungen sowie Akzeptanz von Produkten und Technologien für Frauen. 
Tabelle 11: Die wesentlichen Eckpunkte von FEMtech FTI-Projekten

\begin{tabular}{l|l} 
FEMtech FTI-Projekte & Kurzbeschreibung \\
\hline Ziele & $\begin{array}{l}\text { Akzeptanz und Interesse für das Thema „Gender“ in zukunftsre- } \\
\text { levanten Forschungsfeldern und Produkten unterstützen }\end{array}$ \\
\hline Zielgruppen & $\begin{array}{l}\text { - Forschungs- und/oder technologieintensive Unternehmen } \\
\text { - Universitäten sowie Fachhochschulen }\end{array}$ \\
& $\begin{array}{l}\text { - } \begin{array}{l}\text { außeruniversitäre und kooperative Forschungseinrichtungen, } \\
\text { Kompetenzzentren und -netzwerke }\end{array} \\
\text { - EinzelforscherInnen }\end{array}$ \\
\hline Einreichung & Call-Verfahren: einzelne Organisation oder in Kooperation \\
\hline Förderungsgrundlage & FTE-Richtlinie \\
\hline Laufzeit & mindestens 1 Jahr bis maximal 3 Jahre \\
\hline Förderungshöhe & $\begin{array}{l}\text { maximal } 200.000 € \text { Bundesförderung bei einer Förderungsquote } \\
\text { von maximal } 70 \%, \text { abhängig von der F\&E-Kategorie und der } \\
\text { Unternehmensgröße }\end{array}$ \\
\hline Programmumsetzung & $\begin{array}{l}\text { Einreichberatung und Programmbetreuung: FFG } \\
\end{array}$
\end{tabular}

Quellenangabe: FEMtech Programmdokument; Interviews mit Programm-UmsetzerInnen

FEMtech FTI-Projekte wird im Wettbewerbsprinzip von der FFG ausgeschrieben, wobei insgesamt bis Oktober 2010 zwei Calls in den Jahren 2008 und 2009 mit folgender Projektanzahl stattfanden (ein dritter Call erfolgte im Herbst 2010, die Auswahl der erfolgreichen Projekte wird im März 2011 erfolgen; eingereichte Projekte bleiben hier unberücksichtigt):

Abbildung 15: Übersicht genehmigter und abgelehnter FEMtech FTI-Projekte

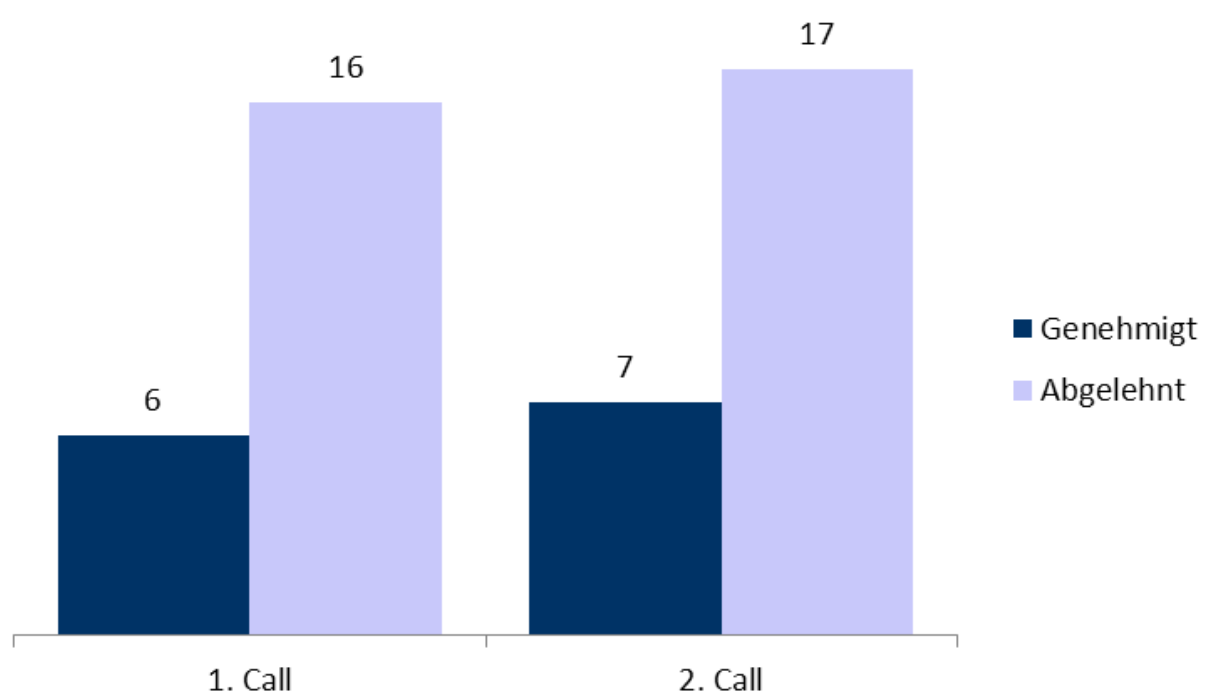

Quellenangabe: FFG-Förderdatenbank (2004 bis Okt. 2010) 
Tabelle 12: Förderquote FEMtech FTI-Projekte

\begin{tabular}{l|l} 
FTI- Projekte & Anzahl \\
\hline Eingereicht & 46 \\
\hline Gefördert & 13 \\
\hline Abgelehnt & 33 \\
\hline Förderquote & $28 \%$
\end{tabular}

Quellenangabe: FFG-Förderdatenbank (2004 bis Okt. 2010)

FEMtech FTI-Projekte zeigt, dass es hier ein Interesse der Community an geförderten Projektvorhaben gibt.

Tabelle 13: Gesamtkosten und Fördervolumina der genehmigten FEMtech FTIProjekte

\begin{tabular}{l|l|l|l} 
& $\begin{array}{l}\text { Gesamt } \\
\text { 1. Call }\end{array}$ & $\begin{array}{l}\text { Gesamt } \\
\text { 2. Call }\end{array}$ & $\begin{array}{l}\text { FTI-Projekte } \\
\text { gesamt }\end{array}$ \\
\hline Gesamtkosten lt. Antrag & $1.345 .583 €$ & $785.763 €$ & $2.131 .346 €$ \\
\hline Bundesförderung & $801.759 €$ & $499.896 €$ & $1.301 .655 €$
\end{tabular}

Quellenangabe: FFG-Förderdatenbank (2004 bis Okt. 2010)

Sechs der 13 geförderten Projekte weisen eine Gesamtdauer von zwei Jahren auf, insgesamt bewegt sich die Projektdauer zwischen 1,5 und drei Jahren.

Die Forschungsprojekte werden z. T. in Kooperation mit WissenschafterInnen weiterer Unternehmen durchgeführt, wie untenstehende Grafik zeigt: vermehrt im 2. Call denn in der ersten Ausschreibung.

Abbildung 16: Projektträger nach Organisationstypen bei genehmigten FEMtech FTI-Projekten

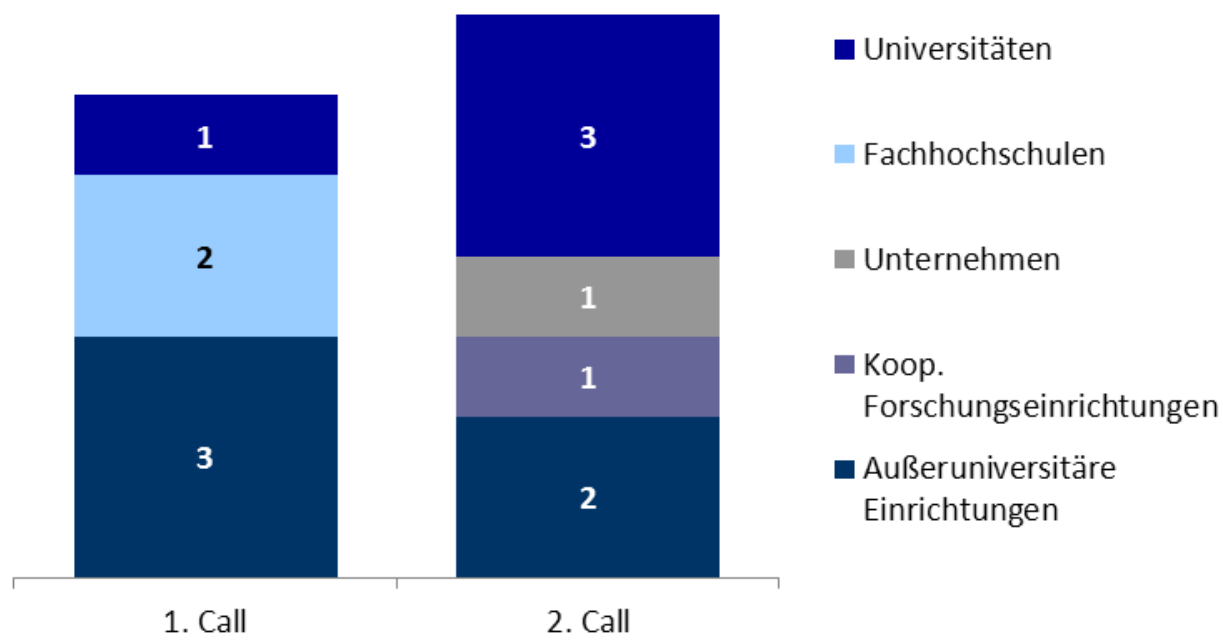

Quellenangabe: FFG-Förderdatenbank (2004 bis Okt. 2010)

Kooperationspartner von FEMtech FTI-Projekten sind großteils Universitäten. Drei Unternehmen beteiligten sich als Partner, hingegen nur eine außeruniversitäre Einrichtung. In der Analyse der abgelehnten Projekte zeigt sich, dass der Anteil an Unternehmen bei den abgelehnten Projekten deutlich höher ist, 
er beträgt rund $30 \%$. Dies lässt vermuten, dass universitäre Einrichtungen (WissenschafterInnen mit Genderwissen) besser in der Lage waren, in dieser Förderlinie gezielt Anträge zu stellen.

Abbildung 17: Projektpartner nach Organisationstypen bei genehmigten FEMtech FTI-Projekten

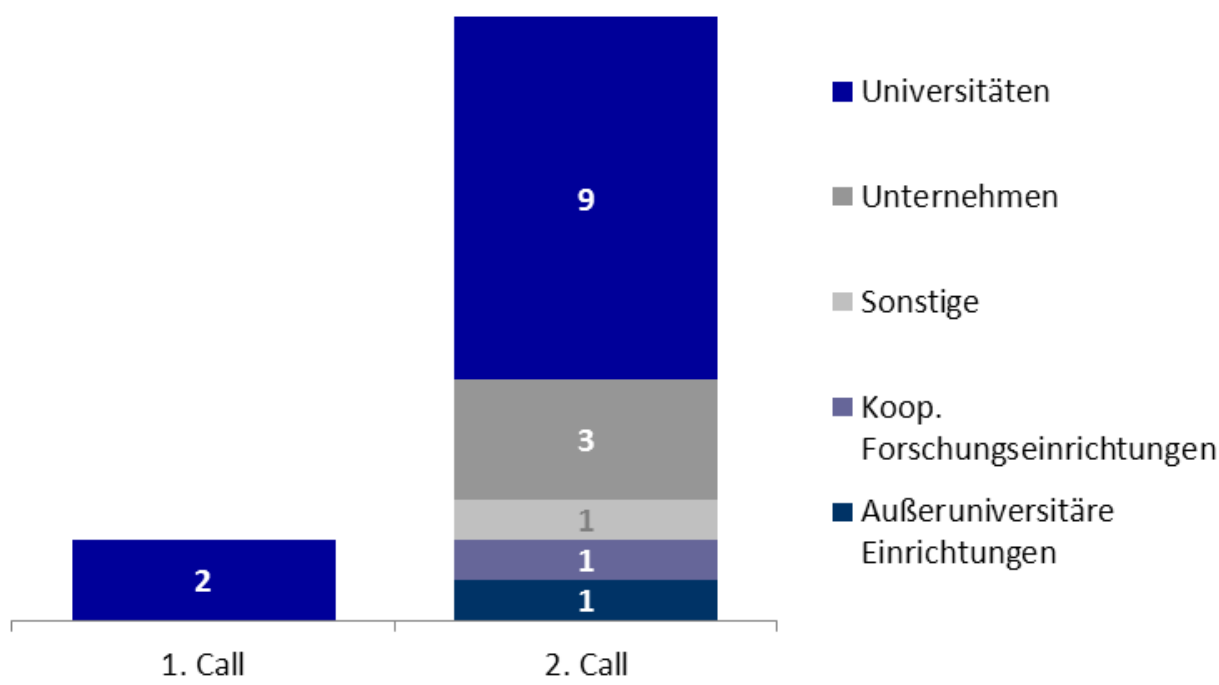

Quellenangabe: FFG-Förderdatenbank (2004 bis Okt. 2010)

Projektpartnerschaften gestalten sich unterschiedlich: Vier FTI-Projekte werden von der Trägerorganisation alleine, fünf in Kooperation mit einem Forschungspartner durchgeführt und die weiteren vier Projekte involvieren zwei bis fünf Partner in ihr Forschungsvorhaben.

Obwohl sich FEMtech FTI-Projekte als einzige Förderlinie auch an EinzelforscherInnen richtet, hat keine Einzelperson daran teilgenommen. Dies erklärt sich aus dem Umstand, dass mindestens $30 \%$ der Gesamtkosten eigenfinanziert werden müssen. 


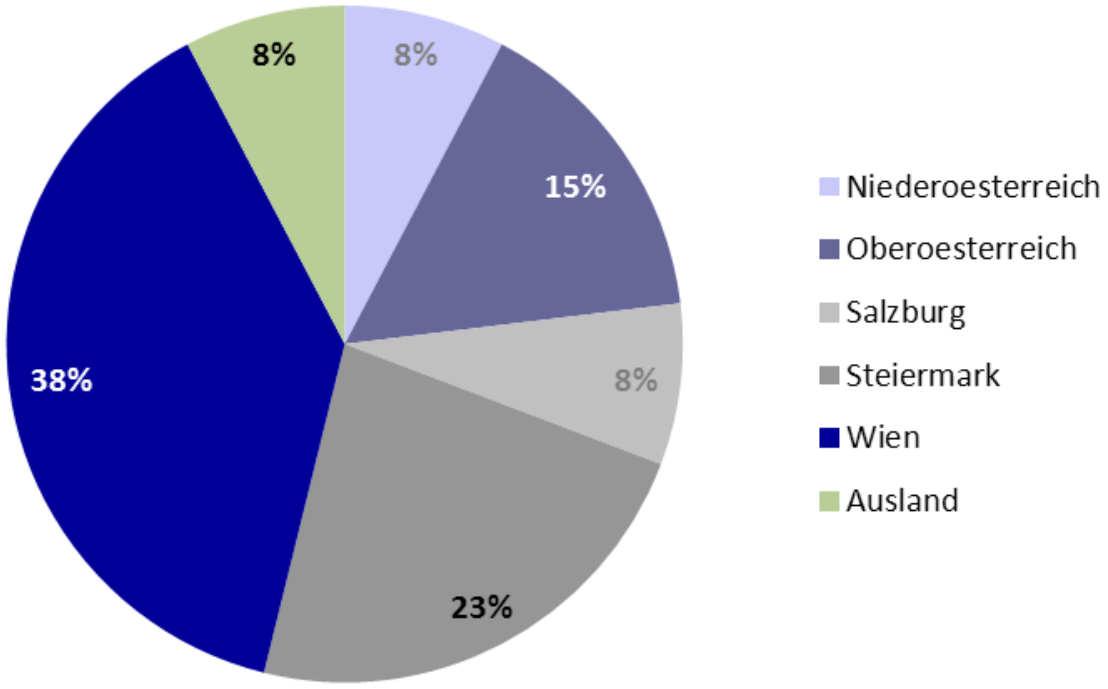

Quellenangabe: FFG-Förderdatenbank (2004 bis Okt. 2010)

FEMtech FTI-Projekte werden zu $38 \%$ in Wien durchgeführt. Die Steiermark und Oberösterreich sind ebenso mit mehreren FTI-Projekten vertreten. Ein Projekt gibt es jeweils in den Bundesländern Niederösterreich und Salzburg sowie eines mit einem deutschen Träger. Forschungseinrichtungen und Unternehmen in den Bundesländern Burgenland, Kärnten, Tirol und Vorarlberg werden mit dieser Förderlinie nicht erreicht. Deren Potenzial im F\&E-Bereich ist jedoch im österreichischen Vergleich insgesamt eher gering, wie die Analyse der Statistik Austria der F\&E-Beschäftigten in den Bundesländern im Jahr 2007 zeigt (vgl. Statistik Austria 2010, S. 157).

Inhaltlich beschäftigen sich die FTI-Projekte mit „Gender“ in der Forschung. Dies passiert auf zwei Ebenen: Berücksichtigung der Genderdimension im gesamten Forschungsprojekt ( $80 \%$ der Projekte) und/oder Differenzierung der ProduktnutzerInnen in Bezug auf Gender (100\% der Projekte). Beinahe alle Projekte haben eine „industrielle Entwicklung“ (EU-Definition) zum Gegenstand.

FEMtech FTI-Projekte spricht mit seinem Forschungsschwerpunkt vor allem den universitären und außeruniversitären Forschungsbereich an. Unternehmen fällt es schwer, Ansatzpunkte für ihre anwendungs- und marktorientierte Forschung zu finden. Die FEMtech Programm-UmsetzerInnen hoffen, dass mit mehr Projekten und Beispielen der Transfer in F\&E-intensive Unternehmen gelingt und hier in Zukunft ebenfalls Projekte starten werden.

\subsubsection{Nutzung der erreichten Zielgruppe}

Die Nutzung der erreichten Zielgruppen bezüglich FEMtech Förderungen wird in zwei unterschiedlichen Bereichen dargestellt. Als Erstes wird gefragt, ob die Projektnehmenden der FEMtech Förderungen in der Struktur anders sind als sonstige FFG-KundInnen. Vereinfacht: Sind FEMtech Fördernehmende „typische“ FFG-KundInnen oder zeigen sich hier Unterschiede? Der zweite Bereich 
betrifft die Bekanntheit der einzelnen FEMtech Förderlinien bei den FEMtech Zielgruppen.

\section{FEMtech Fördernehmende im Vergleich zu sonstigen FFG-KundInnen}

Die Frage, ob die FEMtech Projekte in ihrer Trägerstruktur „typisch“ oder „untypisch“ im Vergleich zu sonstigen FFG-KundInnen sind, ist nicht trivial zu beantworten. Basis dafür war eine bereitgestellte Auswertung der FFG über die Organisationsstruktur aller Projektträger seit 2004, bereinigt um „Kleinstprojektanträge“ wie z. B. Innovationsscheck. Es ist klar, dass eine Mischung von unzähligen Programmen und Zielgruppen ein sehr pauschales Bild wiedergibt, jedoch sollte ermittelt werden, ob chancengleichheitsorientierte Förderungen andere Zielgruppen ansprechen als technologie-, innovations- und forschungsbezogene Förderungsinhalte.

Abbildung 19: Vergleich Organisationsstruktur aller FFG-Programme mit FEMtech Förderlinien

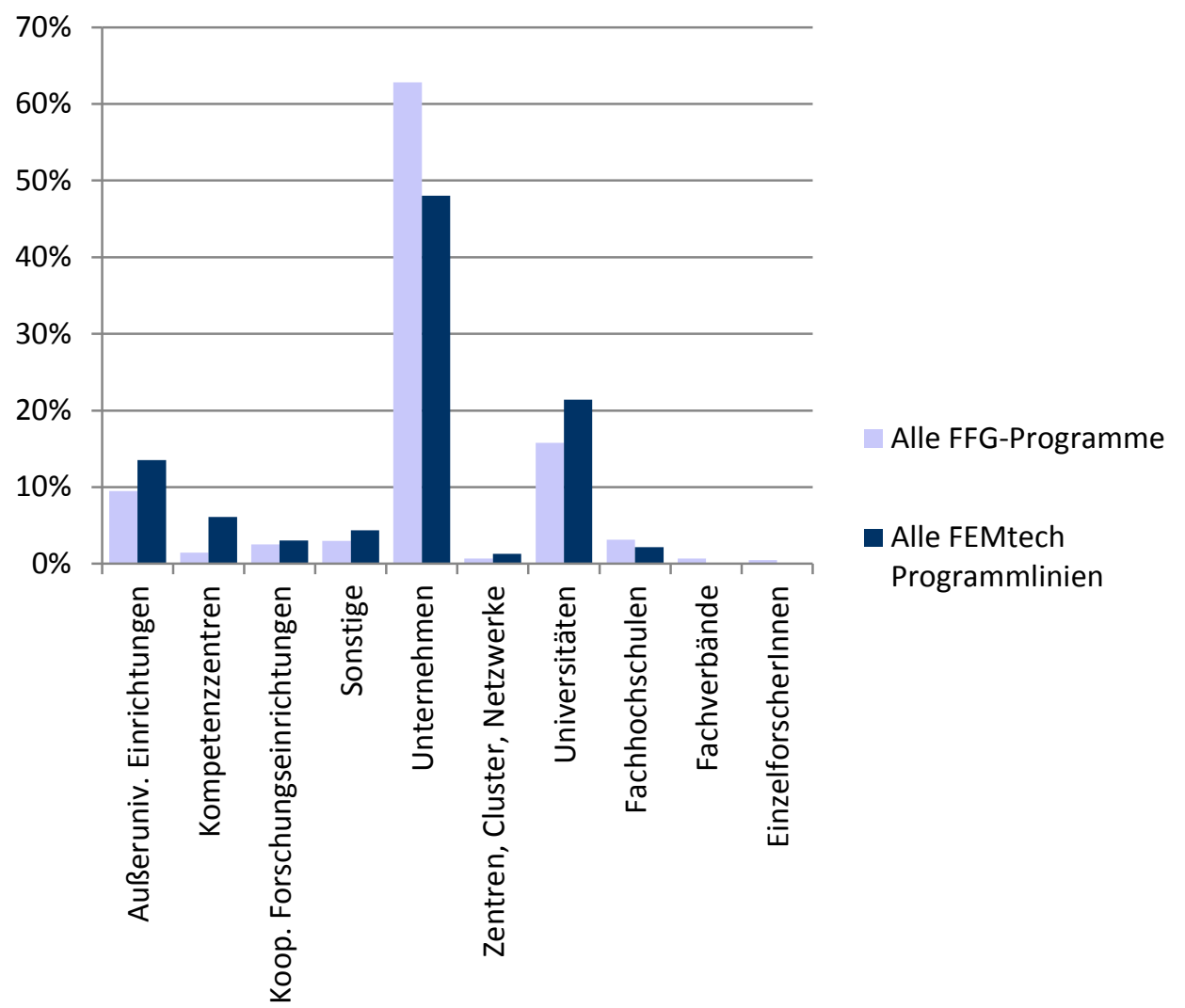

Quellenangabe: FFG-Förderdatenbank Organisationstypen geförderter Unternehmen, Auswertung FFG (2004 bis Oktober 2010) und FFG-Förderdatenbank FEMtech Förderlinien (2004 bis Oktober 2010); jede Institution oder Organisation kann - entsprechend ihrer Anzahl der Projekte in der FFG - mehrfach vertreten sein.

Im Vergleich der Organisationsstrukturen der FFG-Projekte mit Projekten der FEMtech Förderlinien zeigt sich: Unternehmen stellen mit knapp unter $50 \%$ die größte Gruppe der Antragstellenden dar, deren Anteil im Vergleich zu den sonstigen FFG-Förderungen mit über $60 \%$ Unternehmen trotzdem deutlich geringer ist. Die Verschiebung der Anteile kann daraus resultieren, dass sich Universitäten, außeruniversitäre Forschungseinrichtungen und Kompetenzzentren besser für das Thema Chancengleichheit gewinnen lassen bzw. diese hier schon länger Akzente setzen. 
Abbildung 20: Vergleich Bundesländerbeteiligung aller FFG-Programme mit FEMtech Förderlinien

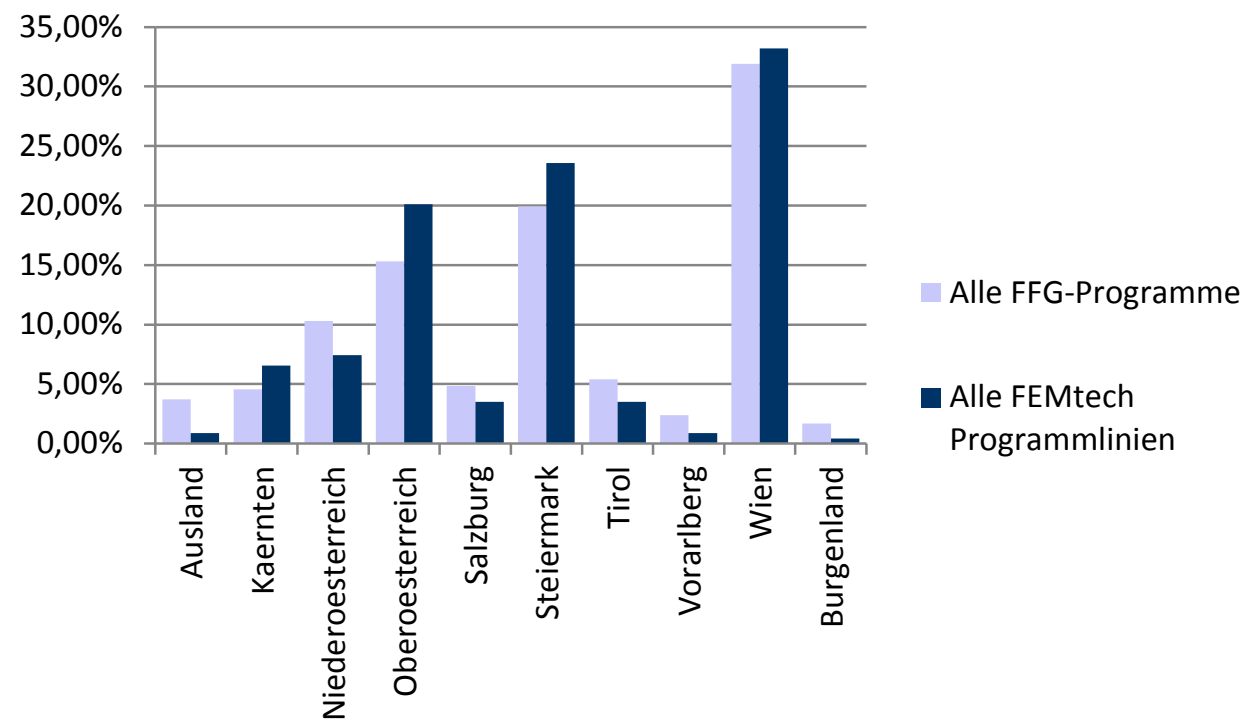

Quellenangabe: FFG-Förderdatenbank Trägerstruktur, Auswertung FFG (2004 bis Oktober 2010) und FFG-Förderdatenbank FEMtech Förderlinien (2004 bis Oktober 2010); jede Institution oder Organisation kann - entsprechend ihrer Anzahl der Projekte in der FFG - mehrfach vertreten sein.

In den FEMtech Förderlinien sind Oberösterreich, die Steiermark, Wien und Kärnten anteilsmäßig stärker vertreten. Oberösterreich sticht hier deutlich hervor, wobei v. a. durch die beiden FEMtech Karrierewege-Projekte inklusive Projektpartner und bei FEMtech Karriere gepunktet wird. Ähnlich verhält es sich mit der Steiermark, die die meisten Projektpartner bei FEMtech Karrierewege aufweist. Auch Kärnten wird gut bedient: in Villach hat „WITEC - the European Association for Women in Science Engineering \& Technology“ seine österreichische Niederlassung und akquiriert hier mit seinen Mitgliedern FEMtech Projekte (so hat z. B. die Fachhochschule Kärnten als einzige FH ein FEMtech Karrierewege-Projekt eingereicht). Burgenland, Salzburg, Vorarlberg und Tirol, schon in der FFG-Grundgesamtheit nicht stark vertreten, werden durch FEMtech kaum erreicht. 
Abbildung 21: Vergleich Unternehmensstruktur aller FFG-Programme und FEMtech Förderlinien

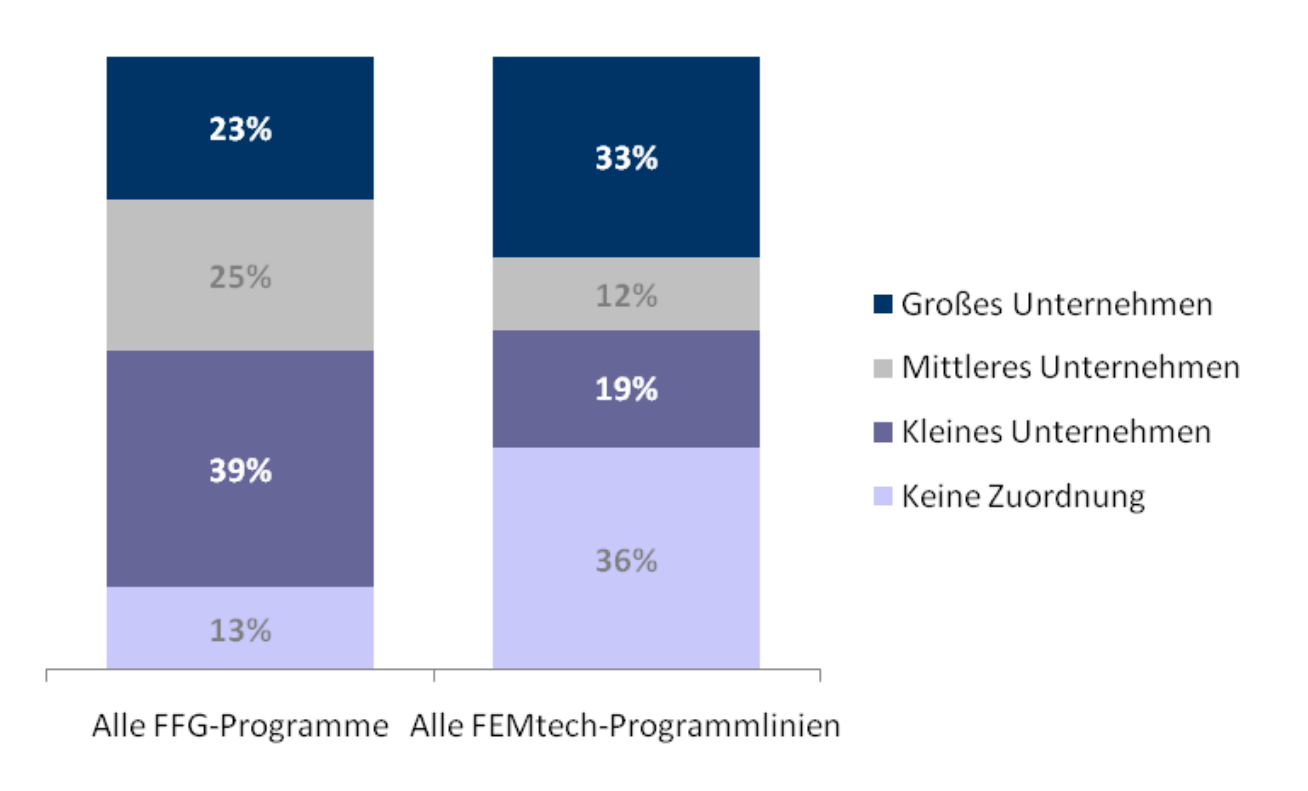

Quellenangabe: FFG-Förderdatenbank Trägerstruktur, Auswertung FFG (2004 bis Oktober 2010) und FFG-Förderdatenbank FEMtech Förderlinien (2004 bis Oktober 2010); jede Institution oder Organisation kann - entsprechend ihrer Anzahl der Projekte in der FFG - mehrfach vertreten sein.

Richtet man den Fokus auf die fördernehmenden Unternehmen, so zeigt sich hier ein deutlicher Unterschied zwischen FEMtech und den anderen FFGProgrammen: Bei FEMtech sind Großunternehmen und Unternehmen mit „keiner Zuordnung“ (Vereine, gemeinnützige Gesellschaften etc.) deutlich stärker vertreten. Hingegen spricht das Programm kaum mittlere Unternehmen und nur in geringem Ausmaß kleine Unternehmen an.

Voraussetzung zur Fördereinreichung ist die Bekanntheit des Programms. Doch wie bekannt sind die einzelnen FEMtech Förderlinien bei ihren Zielgruppen? Erhebungen in der Onlinebefragung zeigen dazu folgendes Bild: 
Abbildung 22: Bekanntheit FEMtech Förderlinien

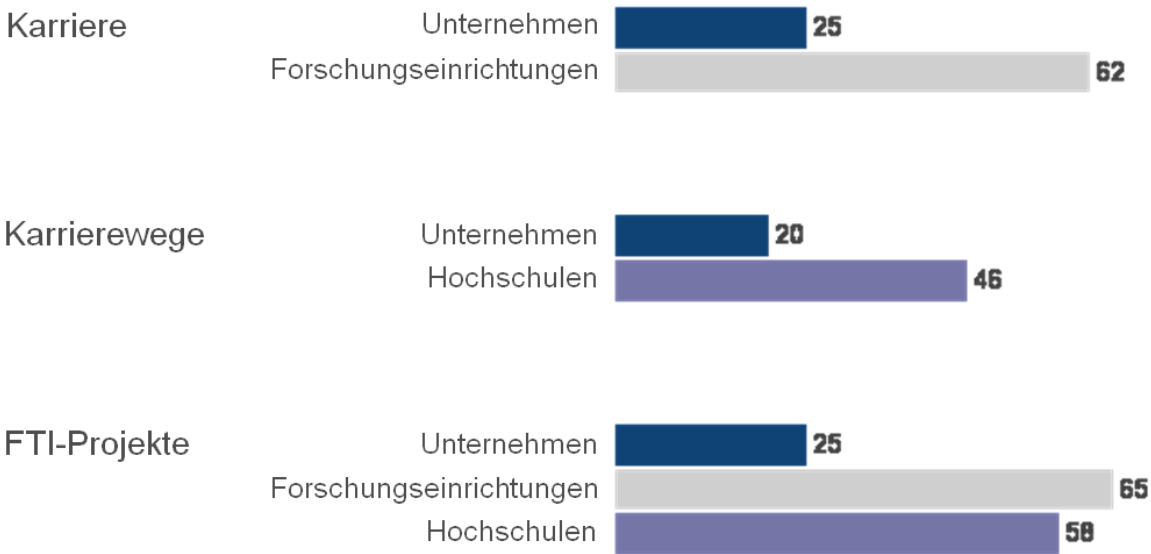

Quellenangabe: ARGE „3C“ basierend auf Onlinebefragung, $\mathrm{n}=1160$ (Unternehmen), $\mathrm{n}=166$ (Forschungseinrichtungen), $\mathrm{n}=342$ (Hochschulen)

Der Bekanntheitsgrad von FEMtech Förderlinien liegt im Unternehmensbereich bei durchschnittlich $23 \%$. Außeruniversitäre Forschungseinrichtungen sind die „best informed players“ bei FEMtech: Mit durchschnittlich $63 \%$ kennen sie die für sie relevanten Förderlinien. Den Hochschulen ist die Förderlinie FEMtech Karrierewege zu 46 \%, FTI-Projekte zu $58 \%$ bekannt. Auch hier zeigt sich: Die direkte Zielgruppe der FTI-Unternehmen ist diejenige, die von FEMtech nicht in dem Maß wie andere Institutionen und Organisationen erreicht wird.

\subsubsection{Zufriedenheit der Zielgruppe}

Die Zufriedenheit der Zielgruppe wurde in der Onlinebefragung und in Interviews ermittelt (die genauen Eckdaten zur Umfrage, wie Anzahl der Befragten, Rücklaufquoten etc., sind im Anhang dargestellt). Generell gilt: Diejenigen, die eine FEMtech Förderung nutzen, sind prinzipiell mit ihr zufrieden. Die Möglichkeit, sich mit dem Thema Frauenförderung und Chancengleichheit zu beschäftigen, wird als sinnvoll erachtet.

Die zweite Frage, wie zufrieden die FEMtech Fördernehmenden mit dem Fördermanagement sind, wird mit „sehr zufrieden“ bis „,zufrieden“ beurteilt - mit einer Ausnahme: FEMtech Karriere. Diese Förderlinie wird in der Antragstellung und in der Berichtspflicht als zu verwirrend und kompliziert erlebt, auch die Transparenz im Auswahlprozess ist für die Fördernehmenden nicht vollständig gegeben. 
Abbildung 23: Zufriedenheit mit FEMtech Fördermanagement

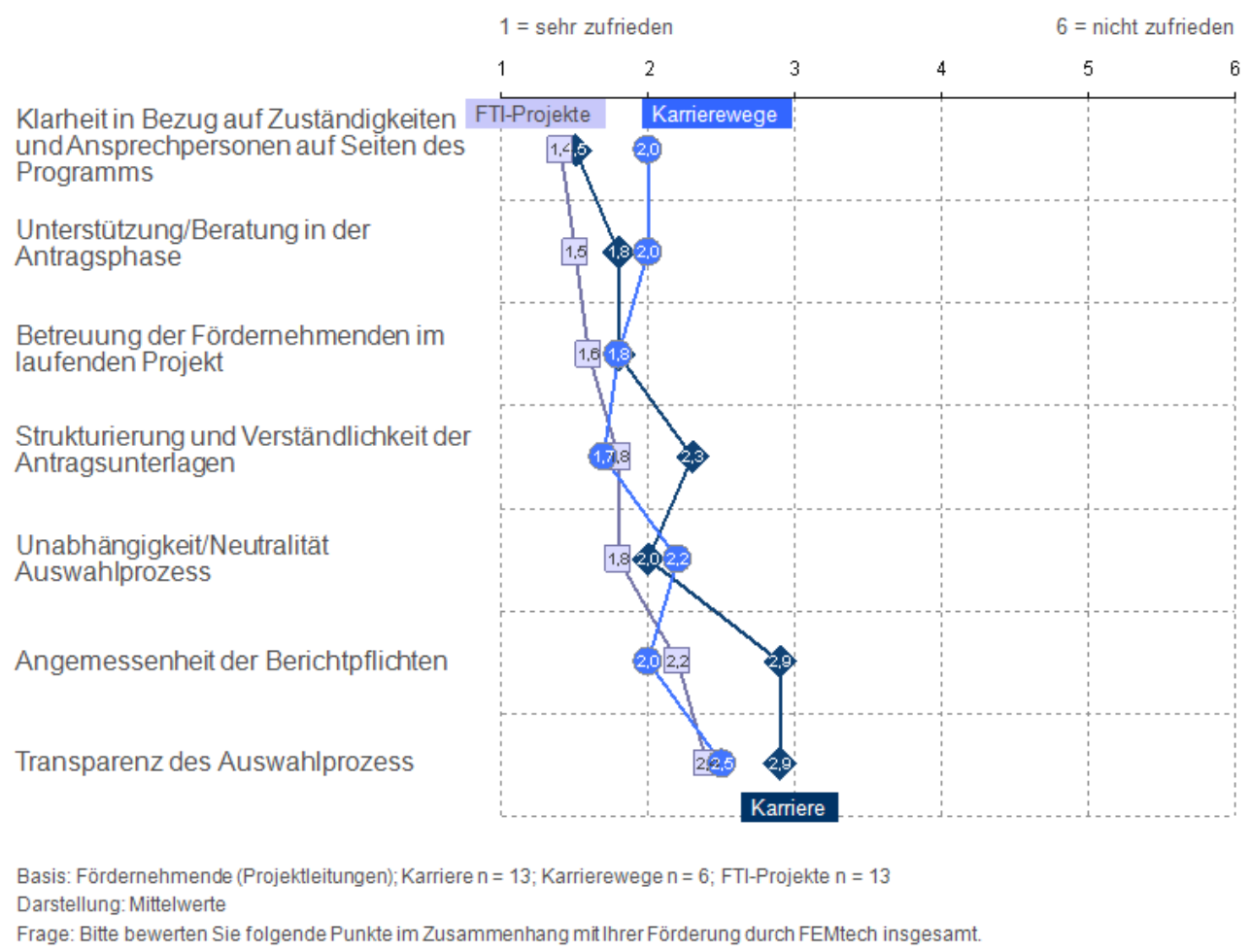

Quellenangabe: ARGE „3C“ basierend auf Onlinebefragung, Fördernehmende (Projektleitungen): FEMtech Karriere $\mathrm{n}=13$, FEMtech Karrierewege $\mathrm{n}=6$, FEMtech FTI-Projekte $\mathrm{n}=13$

Die Ergebnisse der Onlinebefragung werden durch die Interviews bestätigt. FEMtech FTI-Projekte und FEMtech Karrierewege werden in einen „StandardAblauf“ von Forschungsförderungsprojekten eingeordnet und von dieser „Stamm-Klientel“ auch eingereicht. Bedingungen und Prozesse sind vertraut, und FEMtech wird in das gegebene Prozedere gut eingeordnet.

Zum Antragsverfahren, insbesondere zur Programmlinie FEMtech Karriere, merkten die interviewten Unternehmen an, dass dies als „sehr kompliziert“ empfunden wurde und z. T. sogar überlegt wurde, keinen Antrag zu stellen. Diese Bewertung lässt sich nach Art des FEMtech Projektes und der Unternehmensfunktion der fördereinreichenden Person differenzieren:

- FEMtech Karriere - häufig eingereicht von Personalverantwortlichen (Großunternehmen) und GeschäftsführerInnen (KMU): Das Antragswesen ist zu kompliziert und aufwendig (verstehen z. T. die fördertechnische Sprache nicht).

- FEMtech Karrierewege: Einreichende sind Universitäten und Fachhochschulen, die hier ausreichend Erfahrung mit Förderungen haben.

- FEMtech FTI-Projekte (Forschungseinrichtungen): kommen mit dem Antragswesen besser zurecht, da die Logik und Inhalte „ihrer Welt“ entsprechen.

Bezüglich des Berichtswesens zeichnet sich ein ähnliches Muster ab. Interessanterweise merkten einige Personalverantwortliche von Großunternehmen an, dass sie sich nicht vorstellen könnten, dass „sich ein KMU dies jemals antun würde“. Weiters wurde von den interviewten Personen angemerkt: 
- Durchführung des FEMtech Projektes: Den Projektplan zu erfüllen ist schwierig, da sich häufig Dinge im Prozess ändern.

- Die Betreuung ist sehr gut, die Veranstaltungen ebenso.

- In puncto Bericht und Abrechnung heißt es „über Hürden steigen“. Beides erfordert viel Nachfragen, wobei es hier stets freundliche und aufgeschlossene Ansprechpartnerinnen gibt.

\section{Unterstützung und Erweiterung vorhandener Ideen}

Durch FEMtech besteht die Möglichkeit, vorhandene Ideen aufzugreifen und evtl. auch umfassender umzusetzen. Es konnten bestehende Maßnahmen ergänzt und mehr Systematik zum Thema Chancengleichheit im Unternehmen erzielt sowie mehr Transparenz nach innen und außen erzeugt werden. Aus Sicht der befragten Unternehmen und Forschungseinrichtungen, die an FEMtech teilnehmen bzw. teilgenommen haben, hat das Programm auf folgenden Ebenen Veränderungen gebracht:

1. Strategie-Ebene: Wenn Chancengleichheit im Unternehmen bereits Thema war, dann konnte dies durch die FEMtech Förderung forciert und auch besser dargestellt werden.

2. Strukturelle Ebene: Es erfolgten durch FEMtech Verbesserungen insbesondere im Bereich der Bewerbungsverfahren (z. B. Panels männlich und weiblich besetzen) und der Personalrekrutierung. Das zweite wichtige Thema betraf Flexibilisierung der Arbeitszeiten sowie Schaffung der Möglichkeit von Führungsfunktionen mit Teilzeit.

3. Inhaltliche Ebene: „Genderworkshops“ tragen zu einem besseren Verständnis des Themenbereichs im Unternehmen bei.

4. Kulturelle Ebene: Personalverantwortliche in Großunternehmen erwähnten, dass Frauenförderung und Chancengleichheit vermehrt zu einem „Selbstläufer" und damit Teil der Unternehmenskultur werden und auch Nachfolgeprojekte (ohne öffentliche Förderung) geplant sind.

\subsubsection{Verbesserungsvorschläge der Zielgruppe}

Verbesserungsvorschläge beziehen sich einerseits auf den Antragseinreichungsprozess, andererseits auf Rahmenbedingungen zur Einreichung. Bezüglich Antragseinreichung wird für FEMtech Karriere vorgeschlagen, das Antrags-, Berichts- und Abrechnungswesen zu vereinfachen, damit i) ein besserer Zugang für „FFG-Neulinge“ gegeben ist und ii) die Relation zwischen Aufwand und Förderung realistischer wird. FEMtech Karriere verursacht derzeit bei den Projekten einen zu hohen Projektmanagementaufwand, der sich aus unternehmerischer Logik heraus kaum rechtfertigen lässt.

Verbesserungsvorschläge bezüglich FEMtech Förderungen betreffen ebenfalls die Rahmenbedingungen. So sollten insbesondere kleinere Unternehmen besser bei der Einreichung unterstützt werden, ggf. sollte bei ihnen nach Wunsch 
der Zielgruppe auch eine höhere Förderquote genehmigt werden. ${ }^{13}$ Einige FEMtech Fördernehmende regten an, Gender-Expertise in der Forschungsförderung generell stärker zu gewichten (auch in anderen Programmen) und dazu spezifische Trainings anzubieten.

In den Interviews mit dem oberen Management von FTI-Unternehmen und Forschungseinrichtungen wurde erfragt, welche Chancengleichheits- und Frauenförderungsansätze in der Forschungsförderung für sie passend wären. Dabei könnte die österreichische Forschungsförderung aus Sicht der interviewten Unternehmen und Forschungseinrichtungen zum Themenbereich „Chancengleichheit" durchaus offensiver sein, insbesondere in Bezug auf die Sensibilisierung und das Schaffen von Awareness. Hierbei geht es vor allem um die Vermittlung von konkreten Beispielen und das Aufzeigen von Möglichkeiten der positiven Veränderung, die einem „Vollzeitunternehmer schlüssig sind, ohne dass er zum Genderspezialisten werden muss“. Die Wirtschaft sei „nutzenorientiert" (Zitat oMan3), d. h. passende Argumente für Chancengleichheit sollen aufbereitet werden.

Differenziert nach einzelnen Gestaltungsinstrumenten zur Schaffung von mehr Chancengleichheit in der Forschungsförderung gefragt, ergeben sich aus Sicht der interviewten ManagerInnen folgende Möglichkeiten, die in unten stehender Tabelle durch die Sicht der FTI-politischen AkteurInnen ergänzt wird.

\footnotetext{
${ }^{13}$ Selbstverständlich orientieren sich Förderquoten an EU- und Bundesrichtlinien. Hier wird jedoch der Wunsch der Zielgruppe dargestellt, der implizit auch formuliert, dass dies ein Hindernis für KMUs bei FEMtech Fördereinreichungen sein kann.
} 
Tabelle 14: Ansatzpunkte zur Förderung von Chancengleichheit (zusammenfassende Übersicht)

\begin{tabular}{|c|c|c|}
\hline Bereich & $\begin{array}{l}\text { FTI-Unternehmen } \\
\text { und Forschungsein- } \\
\text { richtungen }\end{array}$ & $\begin{array}{l}\text { FTI-politische } \\
\text { AkteurInnen }\end{array}$ \\
\hline $\begin{array}{l}\text { Programme aus- } \\
\text { schließlich für Frauen }\end{array}$ & $\begin{array}{l}\text { nein } \\
\text { tut Frauen nicht gut }\end{array}$ & $\begin{array}{l}\text { nein } \\
\text { nicht exklusiv agieren }\end{array}$ \\
\hline Boni-System & $\begin{array}{l}\text { eher ja } \\
\text { Kriterien sind wichtig }\end{array}$ & $\begin{array}{l}\text { ja } \\
\text { zusätzliche Anreize }\end{array}$ \\
\hline $\begin{array}{l}\text { Neue Bewertungskri- } \\
\text { terien }\end{array}$ & $\begin{array}{l}\text { eher ja } \\
\text { Forschung breiter sehen }\end{array}$ & $\begin{array}{l}\text { vielleicht } \\
\text { sind mühsam, stimulie- } \\
\text { ren jedoch Awareness }\end{array}$ \\
\hline Quotenregelungen & $\begin{array}{l}\text { ja und nein } \\
\text { nicht Zwang }\end{array}$ & $\begin{array}{l}\text { ja und nein } \\
\text { keine klare Richtung } \\
\text { erkennbar }\end{array}$ \\
\hline $\begin{array}{l}\text { Staatliche Auszeich- } \\
\text { nungen }\end{array}$ & $\begin{array}{l}\text { eher nein } \\
\text { Was ist der Mehrwert? }\end{array}$ & $\begin{array}{l}\text { nein } \\
\text { bereits zu viele }\end{array}$ \\
\hline $\begin{array}{l}\text { Gendertrainings für } \\
\text { Forschungsverant- } \\
\text { wortliche }\end{array}$ & $\begin{array}{l}\text { vielleicht } \\
\text { Teilnahme freiwillig }\end{array}$ & $\begin{array}{l}\text { vielleicht } \\
\text { keine Zwangsmaßnahme } \\
\text { + gute Qualität }\end{array}$ \\
\hline Informationsmaterial & $\begin{array}{l}\text { nein } \\
\text { jedoch zielgruppenspe- } \\
\text { zifische Infos ja }\end{array}$ & $\begin{array}{l}\text { nein } \\
\text { Es gibt genug, z.T. zu } \\
\text { detailliert. }\end{array}$ \\
\hline Öffentlichkeitsarbeit & $\begin{array}{l}\text { ja } \\
\text { allerdings breiter anset- } \\
\text { zen }\end{array}$ & $\begin{array}{l}\text { ja } \\
\text { jedoch in Zusammen- } \\
\text { arbeit d. Ministerien }\end{array}$ \\
\hline
\end{tabular}

Quellenangabe: ARGE „3C“, basierend auf Interviews mit den FTI-AkteurInnen und dem oberen Management in FTI-Unternehmen und -Forschungseinrichtungen

FEMtech als ein Programm der Forschungsförderung wird prinzipiell als sinnvoll erachtet, um das Thema Frauenförderung umzusetzen. Chancengleichheit wird mit dem Programm überwiegend noch nicht identifiziert. Dass Chancengleichheit auch ein Thema der Forschungsförderung ist, wird von allen Befragten anerkannt und nicht bagatellisiert. Wünschenswert sei jedoch die breite Einbindung von chancengleichheitsbasierter Forschungsförderung, die in ein Gesamtpaket gesellschaftlicher Rahmenbedingungen passt. „Man kann das Thema nicht einseitig promoten", lautete ein Hinweis dazu. Generell wünscht man ein längerfristiges Durchhalten, da in dem Forschungsförderungsbereich keine schnellen Erfolge erzielbar sind: „Die Leute an den Entscheidungshebeln sollten Mut und Durchhaltekraft haben - und auch die Erfolgskriterien realistisch sehen." 


\subsection{FEMtech Aktivitäten}

\subsubsection{Kurzbeschreibung}

\section{Website und Newsletter}

Die unter www.femtech.at erreichbare Online-Präsenz von FEMtech ist seit 2004 der zentrale Informationsknotenpunkt des Programms. Neben einer Gesamtdarstellung von FEMtech werden hier alle Maßnahmen aus den Bereichen Förderung, Aktivitäten und Wissen abgebildet und erläutert oder in digitaler Form bereitgestellt. Angebote wie die Expertinnen-Datenbank und die im Bereich Wissen gesammelten Daten, Fakten und Publikationen sind zudem nur über die Website erreichbar.

Der online abonnierbare und seit 2004 erscheinende Newsletter informiert quartalsweise über das Programm und seine aktuellen Aktivitäten. Darüber hinaus verweist er auch auf externe Angebote wie Studien, neue Publikationen oder Veranstaltungshinweise. Thematisch bietet er vor allem Informationen zu neuen Kooperationen, Forschungsprojekten mit Gender-Dimension und den Perspektiven von Frauen in naturwissenschaftlich-technischen Berufsfeldern. Des Weiteren werden im Sinne eines Best-Practice berufliche Unterstützungsmaßnahmen für Frauen oder Maßnahmen zur internen Verankerung von Gleichstellung vorgestellt.

\section{Veranstaltungen}

Die seit Start des Programms regelmäßig veranstalteten Netzwerktreffen sind zentrale Termine, auf denen FEMtech über Neuerungen im Programm informiert und aktuelle Trends aufzeigt. Sie dienen dem Informationsaustausch und bieten einen informellen Rahmen für die Vernetzung im Themenbereich Frauen in Forschung und Technologie. Die Analyse der Tagungsprogramme zeigt, dass folgende Inhalte besonders häufig thematisiert werden: Unterstützungsmaßnahmen für Frauen im Beruf (Mentoring, Good Practice, Karriereförderung etc.), Aspekte des Gender Mainstreamings wie Gender Budgeting oder Gender Management sowie Maßnahmen zur Verankerung des Gleichstellungsgedankens in Organisationen. Mit der begleitenden Förderberatung durch die FFG richten sich die Netzwerktreffen explizit auch an potenzielle AntragstellerInnen der drei FEMtech Förderlinien.

Der Gendercocktail ist eine Abendveranstaltung im Vorfeld der Alpbacher Technologiegespräche. Er wird seit 2005 jährlich in Kooperation mit Forschung Austria durchgeführt. Inhaltlicher Schwerpunkt war bis 2009 die Präsentation des aktuellen Gender Booklets, einem Angebot aus FEMtech Wissen, in Verbindung mit einer anschließenden Podiumsdiskussion. Ab 2010 wechselt sich die Vorstellung der nun zweijährlich erscheinenden Publikation mit der neuen Reihe „Brennpunkt“ ab, die für die außeruniversitäre Forschung wichtige Humanressourcen-Aspekte herausgreift.

Unter dem Titel „Forum NaWi)(Tech“ werden jährlich rund zwei Veranstaltungen (in der Regel im Wissenschafts- und Technologiepark Tech Gate Vienna) angeboten, welche Unternehmen und Absolventinnen naturwissenschaft- 
lich-technischer Studiengänge zusammenbringen sollen und die sich als „Job-, Diplomarbeits- und Dissertationsbörsen" verstehen. Mit der Anmeldung können interessierte Studentinnen vorstrukturierte Kurzprofile (Beschreibung zur Person, Interesse am Forum) einreichen, die allen TeilnehmerInnen der Veranstaltung im Vorfeld zur Verfügung gestellt werden, um eine spätere Kontaktaufnahme zu erleichtern.

\section{Preisverleihungen und Auszeichnungen}

Mit der Verleihung von Preisen und Auszeichnungen an Unternehmen und außeruniversitäre Forschungseinrichtungen will FEMtech zur Sichtbarmachung bereits bestehender erfolgreicher Maßnahmen im Bereich Chancengleichheit beitragen und darüber einen Anreiz für die gesamte FTI-Community schaffen, sich mit ihrem Engagement positiv zu präsentieren.

In diesem Sinne vergab das BMVIT 2009 erstmals in sechs Kategorien (jeweils zweckgebundenes Preisgeld in Höhe von $10.000 €$ ) den Staatspreis für Chancengleichheit in F\&E.

Mit dem erstmals 2010 vergebenen Gender Award wurden hingegen gezielt Projekte von Förderungsprogrammen in männlich dominierten Industriebereichen (in diesem Fall der Verkehrs- und Luftfahrtsektor) ausgezeichnet. Insgesamt gingen $85.000 €$ an acht Projekte und eine Studie.

Unter dem Titel FEMtech meets Fiction bündelt das Programm seit 2008 Aktivitäten, welche die mediale Vermittlung weiblicher naturwissenschaftlichtechnischer Rollenbilder und Berufswirklichkeiten fördern. Neben einer entsprechenden inhaltlichen Schwerpunktsetzung in bestehenden Eigenformaten (Netzwerktreffen, genderDiskurs) wurde im Rahmen des vom BMWF (Bundesministerium für Wissenschaft und Forschung) konzipierten Drehbuchwettbewerbes mit dem BMVIT-Sonderpreis „Technikerin im Film“ ein Drehbuch mit einer Technikerin in zentraler Spielfilmrolle ausgezeichnet.

\section{Expertinnen-Datenbank und „Expertin des Monats“}

Die Expertinnen-Datenbank wird seit 2007 in Kooperation mit dem Bundesministerium für Land- und Forstwirtschaft, Umwelt und Wasserwirtschaft (BMLFUW) betrieben und umfasst mit Stand Dezember 2010 rund 1.200 eingetragene Expertinnen, die schwerpunktmäßig in den Bereichen Naturwissenschaften und Technik tätig sind. Als Maßnahme zur Unterstützung, Sichtbarmachung und Vernetzung qualifizierter Forscherinnen richtet sie sich primär an Hochschulen, Forschungseinrichtungen und Unternehmen und bietet die Möglichkeit, nach differenzierten Suchkriterien Wissenschafterinnen für verschiedenste Kooperationsanfragen (Gender Trainings, Begutachtungen, Mentoring, Vorträge etc.) zu recherchieren.

Eng verbunden mit der Expertinnen-Datenbank ist die seit März 2005 durchgeführte monatliche Auszeichnung einer „Expertin des Monats“ durch eine interdisziplinär besetzte Jury. Mit dieser Maßnahme sollen einer breiteren Öffentlichkeit erfolgreiche Wissenschafterinnen und deren Berufsbilder vorgestellt werden, um die Präsenz von Frauen in Wissenschaft und Technik zu stärken und für den potenziellen weiblichen Nachwuchs attraktive Rol- 
len(vor)bilder zu transportieren. Entsprechende Porträts, welche Tätigkeitsbereiche, Forschungsthemen und Lebensläufe der präsentierten Frauen enthalten, erscheinen regelmäßig beim Medienpartner „Der Standard“ als überregionaler Tageszeitung.

\subsubsection{Reichweite und Nutzung insgesamt}

Dieses Kapitel soll basierend auf den Ergebnissen der Online-Befragung zunächst aufzeigen, welche Reichweite und Nutzung FEMtech Aktivitäten insgesamt erzielen, d. h. bezogen auf die Gesamtzielgruppen österreichweit. Dabei wird nach der im engeren Sinne an das Programm gebundenen Community (FEMtech AdressatInnen, also z. B. EmpfängerInnen des Newsletters, von Publikationen, Veranstaltungsbesuchende sowie FEMtech Fördernehmende) und der FTI-Landschaft in der Breite differenziert.

Zunächst zeigt sich ein sehr deutlicher Unterschied in der Bekanntheit und Nutzung von FEMtech Aktivitäten, wenn man den Kreis der FEMtech AdressatInnen mit dem weiteren Kreis von FTI-Unternehmen und -Organisationen vergleicht. So weist der Großteil der Aktivitäten eine Bekanntheit von weit über $50 \%$ in der Gruppe der FEMtech AdressatInnen und -Fördernehmenden auf und wird von dieser auch z. T. intensiv genutzt.

Bei den Befragten aus FTI-Unternehmen und -Organisationen existiert im Falle zentraler Informationsangebote wie der Website zwar eine durchaus bemerkenswerte Sockelbekanntheit (angesichts der Größe und Heterogenität dieser Gruppe) und solide Nutzung, aber darüber hinaus werden weiterführende und stärker inhaltlich ausgerichtete FEMtech Aktivitäten nur sehr sporadisch in Anspruch genommen. Auffällig ist, dass es in beiden Gruppen weitestgehend dieselben Angebote sind, die stärker wahrgenommen bzw. genutzt werden.

Die hohe Bedeutung von Website und Newsletter als zentrale Kommunikations- und Verbreitungsmedien des Programms zeigt sich in erster Linie bei der engeren Community, also FEMtech AdressatInnen und -Fördernehmenden, von denen rund drei Viertel zu den NutzerInnen gehören. Nur das Netzwerktreffen bündelt ähnlich stark den durch das Programm angesprochenen Kreis in dieser Gruppe hat bereits jede(r) Zweite schon einmal daran teilgenommen.

Innerhalb der Angebote der FEMtech Website sticht die ExpertinnenDatenbank mit einer hohen Bekanntheit und auch Nutzung innerhalb der engeren Community hervor. Die hohe Nutzungsrate beinhaltet jedoch vor allem einen hohen Anteil von selbst registrierten Expertinnen (34\%). Hingegen hat nur der kleinere Teil (15\%) innerhalb dieser Gruppe die Datenbank allein zu Recherchezwecken genutzt. In der Gesamtheit der FTI-Landschaft wird die Datenbank dagegen kaum genutzt (3\%).

Ein medial gut platzierbares Vehikel zur Stärkung der Bekanntheit der Expertinnen-Datenbank ist die Expertin des Monats. Die hohe Bekanntheit dieser in „Der Standard“ und auf der FEMtech Website monatlich publizierten Auszeichnung (81\% innerhalb der engeren FEMtech Community und immerhin auch $17 \%$ unter Personen aus FTI-Unternehmen und -Organisationen (FFG)) 
spricht dafür, dass diese medial transportierte Awareness-Maßnahme auch bei den Zielgruppen angekommen ist.

Überraschenderweise sind bei den FTI-Unternehmen und -Organisationen (FFG) FEMtech Veranstaltungen ähnlich bekannt wie die digital bzw. online verfügbaren Angebote - auch wenn Letztere dann konkret häufiger genutzt werden. Formate wie die bereits seit Jahren regelmäßig stattfindenden Netzwerktreffen und Veranstaltungen im Rahmen von Auszeichnungen verfügen innerhalb der FTI-Community über eine solide Bekanntheit und sind damit potenziell geeignet, den Kreis der FEMtech NutzerInnen auf einer persönlichen Ebene zu erweitern - in der Praxis werden die Netzwerktreffen in der Gesamtheit der von der FFG geförderten Organisationen der österreichischen FTI-Landschaft bisher von $2 \%$ genutzt.

Bemerkenswert ist die mit $24 \%$ relativ gute Bekanntheit der FEMtech Auszeichnungen (Gender Award 2010, Staatspreis für Chancengleichheit in F\&E 2009) unter den FTI-Unternehmen und -Organisationen (FFG). Obwohl als Instrument noch relativ jung, sind sie nach der Website das Angebot mit der höchsten Bekanntheit. Trotz präziser Benennung in der Befragung können Fehlzuordnungen aufgrund von Verwechslungen mit anderen Staatspreisen die es in Österreich in einer breiten Vielfalt gibt - hier jedoch nicht vollkommen ausgeschlossen werden. Dies spricht für die prinzipielle Eignung solcher Veranstaltungen für die Schaffung von Aufmerksamkeit, auch wenn die konkrete Teilnahme in Form einer Einreichung nur für einen begrenzten Kreis interessant zu sein scheint.

Abbildung 24: Bekanntheit und Nutzung FEMtech Aktivitäten

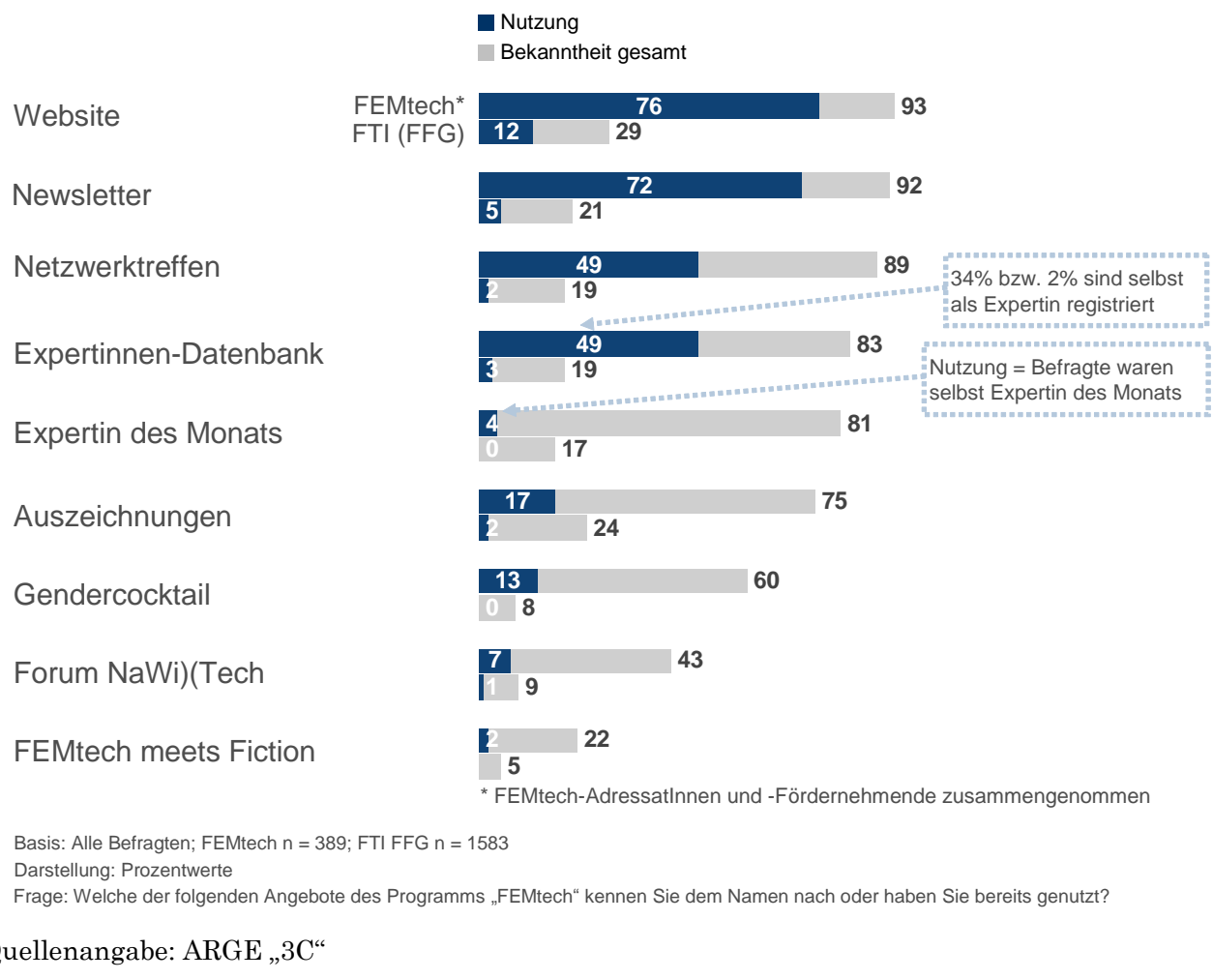




\subsubsection{Nutzung und erreichte Zielgruppen je Aktivität}

Im Unterschied zum vorigen Kapitel, welches Nutzung und Zielgruppenerreichung insgesamt und von einer übergeordneten Warte betrachtet, geht es im Folgenden um die detaillierte Analyse der Zielgruppenerreichung je FEMtech Aktivität. Dazu wird - sofern entsprechende Daten vorliegen - die tatsächliche NutzerInnenstruktur untersucht, basierend auf Anmelde- bzw. Teilnahmelisten (bei den Veranstaltungsformaten), technisch erhobenen Nutzungsdaten (zur Website), gesammelten Medienclippings und aktuellen Datenbank-Dumps (Expertinnen-Datenbank). Insbesondere dann, wenn Daten zur tatsächlichen NutzerInnenschaft nicht vorliegen, werden aber auch Ergebnisse der OnlineBefragung unter FEMtech AdressatInnen und dem weiteren Kreis von FTIUnternehmen und -Organisationen hinzugezogen.

In der Analyse unberücksichtigt bleiben die Formate FEMtech meets Fiction sowie FEMtech Mentoring. ${ }^{14}$

\footnotetext{
${ }^{14} \mathrm{Im}$ Falle von FEMtech meets Fiction bestand die bis dato einzige zielgruppengerichtete Aktivität in der Prämierung eines Drehbuchs mit dem BMVIT-Sonderpreis „Technikerin im Film“. Da jedoch zur Preisverleihungsveranstaltung keine Anmelde- bzw. Teilnahmeliste vorliegt und das Drehbuch bis dato nicht filmisch umgesetzt wurde, kann hier keine Einschätzung zur Zielgruppenerreichung vorgenommen werden. Unter dem Titel FEMtech Mentoring wurden bis dato für die Zielgruppen ausschließlich online Informationen angeboten und auf weitere Mentoring-Angebote verwiesen. Eine konkrete Beratung und Unterstützung durch das Kompetenzzentrum erfolgte hingegen ausschließlich für FördernehmerInnen der Förderlinie „Karrierewege“.
} 


\section{Website}

Tabelle 15: Nutzung FEMtech Website (Anzahl unterschiedlicher BesucherInnen)

\begin{tabular}{|c|c|c|c|c|}
\hline & 2005 & 2006 & 2009 & 2010 \\
\hline Januar & 747 & 1838 & 15 & \\
\hline Februar & 876 & 1675 & 1076 & \\
\hline März & 1392 & 2097 & 4759 & \\
\hline April & 1256 & 1770 & 3964 & 1675 \\
\hline Mai & 1122 & 2367 & 3912 & 1977 \\
\hline Juni & 1145 & 2200 & 4074 & 2186 \\
\hline Juli & 998 & 1661 & 3545 & 1639 \\
\hline August & 1247 & & 3794 & 1895 \\
\hline September & 1347 & & 4222 & 2217 \\
\hline Oktober & 1385 & & 4621 & 2299 \\
\hline November & 1558 & & 5051 & 2753 \\
\hline Dezember & 1181 & & 4023 & 1730 \\
\hline Gesamt & 14.254 & 13.608 & 43.056 & 18.371 \\
\hline
\end{tabular}

Quellenangabe: Statistiken FEMtech Website

Bei der Interpretation der Nutzungsdaten der Website ist zu berücksichtigen, dass für zwei Zeiträume (08/2006 - 12/2008 und 01/2010 - 03/2010) keine monatlichen Nutzungszahlen vorliegen und Anfang 2009 zudem ein Relaunch der Website erfolgte. Dieser dürfte verantwortlich sein für die im Vergleich zu 2005 und 2006 stark gestiegene Nutzung, da der Neustart nicht nur über existierende Verteiler wie z. B. den FEMtech Newsletter beworben wurde, sondern auch mit der Bereitstellung neuer Inhalte bzw. Angebotsrubriken einherging (darunter der Bereich „Wissen“).

Beim Vergleich der Jahre 2009 und 2010 ist zu bedenken, dass seit April 2010 die Auswertung mit einem neuen Webanalyse-Werkzeug (Google Analytics) erfolgt. Die starke Diskrepanz zwischen beiden Jahren lässt vermuten, dass 2010 „konservativer“ gezählt wurde und die tatsächliche Nutzung in beiden Jahren näher beieinanderliegt.

Die Nutzungsdaten der Website im Jahr 2010 lassen bereits vermuten, dass es eine große Überschneidung zu den Newsletter-AbonnentInnen gibt (im Durchschnitt rund 2.500), was durch die Ergebnisse der Online-Befragung bestätigt 
wird. Dort haben $76 \%$ derjenigen bereits die Website besucht, die durch FEMtech regelmäßig über das Programm informiert werden (z. B. per Newsletter) oder bereits Offline-Angebote des Programms (z. B. Teilnahme an Veranstaltungen) genutzt haben. Innerhalb der weiteren FTI-Community (die noch nicht aktiv durch FEMtech adressiert wurde) sind zwar Bekanntheit (29\%) und damit auch die Nutzung (12\%) der Website deutlich niedriger; angesichts der Diversität der gesamten FTI-Landschaft lässt sich die Frequentierung der Website hier aber immer noch als sehr solide bewerten.

Letzteres mag auch damit zusammenhängen, dass die Website Informationen für potenzielle Antragstellende der FEMtech Förderlinien bereithält. Wie die Analyse der Nutzung einzelner Seitenbereiche für das Jahr 2010 zeigt, ist die Einstiegsseite in diesen Unterbereich die am häufigsten geklickte. Weitere gut genutzte Seitenbereiche sind die Expertinnen-Datenbank und die damit verbundenen Expertinnen des Monats. Auch Informationen zu einzelnen Veranstaltungsformaten, insbesondere den Netzwerktreffen, werden häufig angeklickt und intensiv betrachtet. Die Wissensangebote (Daten, Publikationen etc.) werden hingegen seltener genutzt.

Eine separate Befragung von NutzerInnen der FEMtech Website während ihres Besuchs (per On-Site-Rekrutierung) bestätigt das Aktivierungspotenzial der Website für eine weitergehende Beschäftigung mit FEMtech. So planen $66 \%$, zukünftig FEMtech Veranstaltungen zu besuchen bzw. sich dazu weiter zu informieren und $47 \%$ haben vor, in der Datenbank eine Expertin zu suchen bzw. sich selbst als Expertin zu registrieren. Hierbei ist allerdings zu berücksichtigen, dass trotz einer Feldphase von annähernd zwei Monaten lediglich 40 Personen an der On-Site-Befragung teilgenommen haben und dementsprechend kein repräsentatives NutzerInnenbild ergeben. Es ist zu vermuten, dass hier vor allem eng an das Programm gebundene Personen teilgenommen haben.

Die Ergebnisse von Online- und On-Site-Befragung sowie die mit Ausnahme saisonaler Ausreißer konstante Zahl von monatlich 2.000 unterschiedlichen BesucherInnen für das Jahr 2010 passt zum Bild, dass die Website als umfassendes Informations- und Rechercheangebot vor allem die bereits enger mit dem Programm verbundenen Zielgruppen bindet und darüber hinaus in kleinerem Umfang für breitere Zielgruppen als Einstiegsportal ins FEMtech Programm dient.

\section{Newsletter}

Der Kreis der EmpfängerInnen des FEMtech Newsletters liegt laut Versandstatistiken des Kompetenzzentrums seit 2009 (zu den vorigen Jahren liegen keine Daten vor) konstant bei rund 2.500 Personen.

Ergänzt durch die Ergebnisse der Online-Befragung zeigt sich, dass der Newsletter vor allem von Personen rezipiert wird, die bereits viele FEMtech Angebote nutzen und sich dadurch auf dem Laufenden halten wollen. Gerade die befragten VertreterInnen aus Medien, Politik, von Förderorganisationen oder Verbänden gehören fast geschlossen zum AbonnentInnenkreis. Trotzdem spricht der Newsletter - jedoch auf einem niedrigeren Niveau als die Website auch sporadisch NutzerInnen gut an. Laut Onlinebefragung nutzen $6 \%$ der 
privatwirtschaftlichen Unternehmen, welche von der FFG insgesamt durch Fördermaßnahmen erreicht werden, den Newsletter. Auch aufgrund der mittlerweile tief verzweigten Struktur der Website geben einige Teilnehmende an der Online-Befragung an, sich ausschließlich per Newsletter über FEMtech Neuigkeiten zu informieren und die Website nur noch selten oder gar nicht mehr zu besuchen.

\section{Netzwerktreffen}

Tabelle 16: Teilnahmen bzw. Teilnehmende Netzwerktreffen

\begin{tabular}{l|c|c|c|c|c|c|c|c} 
& $\mathbf{2 0 0 4}$ & $\mathbf{2 0 0 5}$ & $\mathbf{2 0 0 6}$ & $\mathbf{2 0 0 7}$ & $\mathbf{2 0 0 8}$ & $\mathbf{2 0 0 9}$ & $\mathbf{2 0 1 0}$ & Gesamt \\
\hline Teilnahmen gesamt & 165 & 190 & 238 & 242 & 520 & 363 & 355 & 2073 \\
\hline $\begin{array}{l}\text { Davon unterschiedliche } \\
\text { Teilnehmende }\end{array}$ & 135 & 148 & 176 & 240 & 391 & 299 & 281 & 1221 \\
\hline $\begin{array}{l}\text { Durchschnittsanzahl } \\
\text { Teilnehmende pro Ver- } \\
\text { anstaltung }\end{array}$ & 55 & 63 & 60 & 61 & 87 & 91 & 118 & 77 \\
\hline
\end{tabular}

Quellenangabe: Basis: 10 Anmelde- und 16 Anwesenheitslisten, Anzahl Anmeldungen zu einer Veranstaltung; 27 Veranstaltungen

Von 2004 bis Juni 2010 haben an den Netzwerktreffen insgesamt 1.221 verschiedene Personen ${ }^{15}$ teilgenommen, was einer hohen Reichweite dieses Formats in der themenaffinen und stark auf Wien konzentrierten (wo fast alle Netzwerktreffen stattfanden) Community entspricht. Im Abgleich mit den Ergebnissen der Onlinebefragung (vgl. Kapitel 3.2.2 „Reichweite und Nutzung insgesamt") wird deutlich: Der Kern themenaffiner Personen hat mit über 1.200 Personen zwar eine respektable Größe, bezogen auf die von der FFG insgesamt erreichten AkteurInnen der österreichischen FTI-Landschaft ist er aber dennoch klein (nur $2 \%$ aller Befragten haben ein Netzwerktreffen besucht).

Die Bedeutung der Veranstaltung hat dabei stetig zugenommen; dafür spricht die Entwicklung der durchschnittlichen TeilnehmerInnenzahl pro Veranstaltung, die sich in den zurückliegenden sechs Jahren mehr als verdoppelt hat. Entscheidende Faktoren für den sprunghaften Anstieg nach 2007 waren die Einrichtung des Kompetenzzentrums im Folgejahr sowie insbesondere die ersten Calls der 2008 aufgelegten Förderlinien „Karrierewege“ und „FTI-Projekte“ und die Verdoppelung der Förderhöhe bei „Karriere“, in deren Zuge viele Teilnehmende den Besuch von Netzwerktreffen für eine persönliche Förderberatung genutzt haben dürften.

Wie die hohe Zufriedenheit aller Veranstaltungs-NutzerInnen zeigt (Mw. 2,1 auf 6er-Skala; zur Zufriedenheit im Detail vgl. Kapitel 3.2.4 „Zufriedenheit der Zielgruppen“), konnte über die Jahre eine intensive Vernetzung vorangetrieben werden, wobei die Veranstaltung stetig um neue Personen erweitert wurde. Dies belegt die Relation von unterschiedlichen TeilnehmerInnen (erreichte

\footnotetext{
${ }_{15}$ Die Gesamtzahl der unterschiedlichen Teilnehmenden bezieht sich auf alle Jahre und stellt keine kumulierte Summe dar, da in den Jahresspalten nur die unterschiedlichen Teilnehmenden für das jeweilige Jahr angegeben sind (unabhängig davon, ob sie nur in diesem oder auch in anderen Jahren Veranstaltungen besucht haben).
} 
Personen unabhängig davon, ob sie mehrere oder nur eine Veranstaltung besucht haben) zur Gesamtzahl: Durchschnittlich hat jede(r) Teilnehmende 1,7 Netzwerktreffen von insgesamt 27 besucht. Der Anteil von Einmalbesuchenden beträgt dabei $70 \%$ und nur wenige Personen (insgesamt $8 \%$ ) haben mehr als 3 Termine besucht. Dass sich über die Jahre kein „harter Kern“ von Wiederholungsbesuchenden gebildet hat, hängt mit den für jedes Treffen anders gesetzten thematischen Schwerpunkten zusammen, die jeweils verschiedene Teile der am Thema Frauen in Forschung und Technologie interessierten Community ansprechen. Die insbesondere von TeilnehmerInnen aus FTI-Unternehmen in der Online-Befragung geäußerte Wahrnehmung, dass sich auf den Netzwerktreffen ein „eingeschworener Kreis“ bzw. „eine enge Community“ trifft (vgl. Kapitel 4.3.2 „FEMtech Wirkungen: Aktivitäten“), liegt also eher in der generell starken Bindung vieler Besuchenden an FEMtech und einem über viele Jahre kultivierten Interesse an Gleichstellungsthemen begründet.

Etwas mehr als die Hälfte der Teilnahmen entfallen auf forschungs- und technologieintensive Unternehmen oder Organisationen, wobei privatwirtschaftliche Unternehmen hier ähnlich stark vertreten sind wie (Fach-) Hochschulen und außeruniversitäre Forschungseinrichtungen. Neben diesen Zielgruppen der FEMtech Förderlinien (zu denen die Netzwerktreffen Beratungsangebote bereitstellen) nehmen des Weiteren auch Politik / öffentliche Verwaltung, Förderorganisationen und Beratungsunternehmen in wahrnehmbarem Maß an den Veranstaltungen teil. 
Tabelle 17: Teilnehmende Netzwerktreffen - nach Organisationszugehörigkeit

\begin{tabular}{lc} 
& Anteil \\
\hline Teilnehmende gesamt (Basis) & 2073 \\
\hline Forschungs-/technologieintensives Unternehmen oder Organisation & $53 \%$ \\
\hline darunter: & \\
Privatwirtschaftliches Unternehmen & $32 \%$ \\
Außeruniversitäre und kooperative Forschungseinrichtung & $27 \%$ \\
Universität/Akademie & $27 \%$ \\
Fachhochschule & $7 \%$ \\
Kompetenzzentrum oder -netzwerk & $6 \%$ \\
Verein & $1 \%$ \\
Intermediäre Einrichtung (Technologiezentrum, Clustermanagement etc.) & $1 \%$ \\
\hline Politik / öffentliche Verwaltung & $8 \%$ \\
\hline FEMtech Kompetenzzentrum & $6 \%$ \\
\hline Beratungsunternehmen & $6 \%$ \\
\hline Forschungs- oder Wirtschaftsförderungsorganisation & $6 \%$ \\
\hline MedienvertreterIn & $3 \%$ \\
\hline Verband / Interessenvertretung & $1 \%$ \\
\hline StudentIn & $0 \%$ \\
\hline Sonstige Organisation & $5 \%$ \\
\hline Sonstiges & $3 \%$ \\
\hline keine Angabe & $7 \%$ \\
\hline
\end{tabular}

Quellenangabe: Basis: Anmelde- bzw. Anwesenheitslisten zu 27 Veranstaltungen

\section{Gendercocktail}

Tabelle 18: Anmeldungen Gendercocktail

\begin{tabular}{|c|c|c|c|c|c|c|}
\hline & 2007 & 2008 & 2009 & $\Sigma$ & $\begin{array}{c}\text { Davon } \\
\text { verschie- } \\
\text { dene TN }\end{array}$ & $\varnothing$ \\
\hline $\begin{array}{l}\text { Anzahl } \\
\text { Teilnehmende }\end{array}$ & 48 & 68 & 156 & 272 & 228 & 91 \\
\hline
\end{tabular}

Quellenangabe: Basis: 3 Anmeldelisten zu 3 Veranstaltungen

Die Beurteilung der TeilnehmerInnenzahlen der Gendercocktails wird erschwert durch die enge Anbindung an die Alpbacher Technologiegespräche, in deren Rahmen sie am Vorabend stattfinden. Zudem liegen für die Gendercocktails 2007 bis 2009 nur Anmeldelisten vor. Ungeachtet einer generellen Vermischung des Publikums beider Veranstaltungen lässt sich jedoch neben einer gestiegenen Zahl von Anmeldungen festhalten, dass es eine geringe Zahl von Wiederholungsanmeldungen gibt. Dies mag damit zusammenhängen, dass die Themenschwerpunkte sich von Jahr zu Jahr stark unterscheiden (2009 ging es bspw. um nicht-traditionelle Karriereverläufe forschender Männer).

In der Zusammensetzung des TeilnehmerInnenfeldes zeigt sich - im Vergleich zu den stärker zielgruppenorientierten Netzwerktreffen - eine starke Präsenz 
des politisch-administrativen Sektors. AkteurInnen aus Politik, Verwaltung und Förderorganisationen sind genauso stark vertreten wie FTI-Unternehmen und -Organisationen. Die wissenschaftspolitische Ausrichtung der Alpbacher Technologiegespräche spiegelt sich letztlich auch in der geringen Teilnahme privatwirtschaftlicher Unternehmen an den Gendercocktails.

Tabelle 19: Teilnehmende Gendercocktail - nach Organisationszugehörigkeit

Anteil

\begin{tabular}{lc} 
Teilnehmende gesamt (Basis) & $\mathbf{2 7 2}$ \\
\hline Forschungs-/technologieintensives Unternehmen oder Organisation & $35 \%$ \\
\hline darunter: & \\
Außeruniversitäre und kooperative Forschungseinrichtung & $57 \%$ \\
Privatwirtschaftliches Unternehmen & $17 \%$ \\
Universität & $17 \%$ \\
Sonstige Organisationen & $9 \%$ \\
\hline Politik / öffentliche Verwaltung & $21 \%$ \\
\hline Forschungs- oder Wirtschaftsförderungsorganisation & $13 \%$ \\
\hline Beratungseinrichtung & $7 \%$ \\
\hline MedienvertreterIn & $2 \%$ \\
\hline Verband / Interessenvertretung & $2 \%$ \\
\hline Sonstige Organisation & $3 \%$ \\
\hline Sonstiges & $3 \%$ \\
\hline k. A. & $15 \%$ \\
\hline
\end{tabular}

Quellenangabe: Basis: 3 Anmeldelisten zu 3 Veranstaltungen

\section{Forum NaWi)(Tech}

Tabelle 20: Teilnehmende Forum NaWi)(Tech

\begin{tabular}{|c|c|c|c|c|c|c|c|c|c|c|}
\hline & \multicolumn{2}{|c|}{2006} & \multicolumn{2}{|c|}{2007} & \multirow{2}{*}{$\begin{array}{c}2008 \\
5 .\end{array}$} & \multicolumn{2}{|c|}{2009} & \multirow{2}{*}{$\begin{array}{c}2010 \\
8\end{array}$} & \multirow[t]{2}{*}{$\Sigma$} & \multirow[t]{2}{*}{$\varnothing$} \\
\hline & 1. & 2. & 3. & 4. & & 6. & 7. & & & \\
\hline $\begin{array}{l}\text { Studentinnen/ } \\
\text { Absolventinnen }\end{array}$ & 17 & 23 & 46 & \multirow{4}{*}{ k. A. } & 48 & 27 & 20 & 25 & 206 & 29 \\
\hline Privatwirtschaftliche Unternehmen & 7 & 12 & 11 & & 9 & 7 & 8 & 9 & 63 & 9 \\
\hline Außeruniversitäre/ kooperative FE & 2 & 1 & & & & 4 & & 3 & 10 & 1 \\
\hline Universität & & & & & & 1 & & & 1 & - \\
\hline
\end{tabular}

Quellenangabe: Basis: 5 Anwesenheits- und 2 Anmeldelisten; 7 Veranstaltungen

Im Verhältnis von teilnehmenden Unternehmen (die jeweils durch mehrere Personen repräsentiert waren) zu anwesenden Studentinnen und Absolventinnen zeigt sich das auch seitens der Programm-UmsetzerInnen angesprochene Problem der Akquise des weiblichen Nachwuchses für dieses Format. So stehen im Durchschnitt zwei interessierte Nachwuchsfrauen einem unternehmerischen Präsentationsstand gegenüber. Angesichts dieser geringen Resonanz 
erscheint die regelmäßige Ausrichtung der Veranstaltung im Wissenschaftsund Technologiepark Tech Gate Vienna überdimensioniert.

Passend zur eingeschränkten Nutzung des Forum NaWi)(Tech durch die Zielgruppen beschränkt sich die Medienresonanz auf sieben Beiträge seit 2006.

\section{Preisverleihungen und Auszeichnungen}

Für den 2009 erstmals durch das BMVIT vergebenen Staatspreis für Chancengleichheit in F\&E gab es insgesamt 33 Einreichungen: 18 von Unternehmen und 15 von außeruniversitären Forschungseinrichtungen. Davon wurden letztlich 14 Einreichungen nominiert für die Vergabe des Preises in sechs nach Organisationsgröße unterschiedenen Kategorien.

Auffällig ist, dass es im Verhältnis zu den vergebenen Preisen proportional weniger Einreichungen durch Unternehmen (18 Einreichungen für 4 Preise) als Forschungseinrichtungen (15 Einreichungen für 2 Preise) gab.

Der erstmals 2010 vergebene Gender Award wurde hingegen gezielt an Projekte der Förderprogramme „Intelligente Verkehrssysteme und Services plus“ (I2V) und TAKE OFF verliehen. 65 bestehende Projekte wurden nach der Förderzusage per E-Mail zur Teilnahme am Preis aufgefordert. Dafür war keine gesonderte Einreichung erforderlich, da die Begutachtung allein auf Basis des bestehenden Projektantrags erfolgte. Trotz dieser damit sehr geringen Hürde wurden für eine Teilnahme in der Kategorie 1 (Projekte mit weiblicher Projektleitung und/oder überdurchschnittlichem Forscherinnenanteil) nur acht Projekte gewonnen und vier für die Kategorie 2 (Projekte mit spezifischer Genderausrichtung in den Forschungsinhalten). Verliehen wurde der Preis letztlich an sechs Projekte in Kategorie 1 und zwei in Kategorie 2, wobei ein Projekt zweifach ausgezeichnet wurde.

Dass damit die Teilnahme am Gender Award hinter den Erwartungen zurückblieb, zeigt sich bereits darin, dass das für die Prämierung bereitgestellte Budget von $250.000 €$ nur zu einem Drittel ausgeschöpft wurde. Laut einer nicht repräsentativen Feedback-Einholung bei Nicht-Einreichenden durch das Kompetenzzentrum fühlten sich zuständige Projektleitungen entweder nicht angesprochen oder waren sich ihrer potenziellen Chancen nicht bewusst.

Mit den Preisverleihungsveranstaltungen zu Staatspreis und Gender Award wurde schließlich das Interesse von jeweils rund 160 Personen (laut Anmeldelisten) geweckt. Dass unter den Anmeldungen bei beiden Auszeichnungen FTIUnternehmen und -Organisationen dominieren, überrascht wenig, da diese als (potenzielle) Preisträger im Fokus stehen (insgesamt über beide Auszeichnungen hinweg 80 Anmeldungen aus Unternehmen, 60 aus Forschungseinrichtungen und 17 aus Universitäten). Daneben sind aber auch die politische Ebene als Ausrichter (42 Anmeldungen) und Medien (22 Anmeldungen) gut vertreten.

Obwohl auf beiden Veranstaltungen MedienvertreterInnen in ähnlichem Umfang angemeldet waren, muss angesichts von letztlich 16 Medien-Beiträgen zum Staatspreis und lediglich vier zum Gender Award von einer geringeren Attraktivität des Gender Awards für die Berichterstattung ausgegangen werden. 
Insgesamt betrachtet, bestätigt sich das Potenzial von Auszeichnungen und den damit verbundenen Preisverleihungsveranstaltungen für eine öffentlichkeitswirksame Schaffung von Aufmerksamkeit. Allerdings zeigt sich deutlich, dass Unternehmen und Organisationen schwer zu mobilisieren (siehe oben: trotz extrem geringer Einreichhürden und umfangreicher Akquise- und Nachfassbemühungen ließen sich beim „Gender Award“ nur sehr wenige zu einer Teilnahme bewegen) und eher über das Thema Chancengleichheit als Gender anzusprechen sind - was sich letztlich auch im Medieninteresse widerspiegelt.

\section{Expertinnen-Datenbank und Expertin des Monats}

Mit 1.220 eingetragenen Expertinnen deckt die Expertinnen-Datenbank ein breites Spektrum unterschiedlicher Fachbereiche in Naturwissenschaft und Technik ab. Wie eine Auswertung der Datenbank (Stand: Dezember 2010) zeigt, sind die meisten dieser Fachfrauen im universitären Bereich tätig (36 \%). Aber auch außeruniversitäre Forschungseinrichtungen und Unternehmen aus Industrie und Wirtschaft, in denen jeweils ein Fünftel der Expertinnen beschäftigt ist, sind umfangreich in der Datenbank vertreten. Der hohe Anteil freiberuflicher Expertinnen (14\%) kann zudem als Indiz dafür gelten, dass die Expertinnen-Datenbank aus Sicht der Fachfrauen als Kontaktbörse und damit Akquise-Instrument in eigener Sache verstanden wird.

Von den registrierten Expertinnen geben $12 \%$ an, schon einmal eindeutig aufgrund der Datenbank angefragt worden zu sein; weitere $9 \%$ können dies aufgrund nicht rückführbarer Anfrage(n) zumindest nicht ausschließen.

Noch stärker als bei anderen FEMtech Aktivitäten zeigt sich in der Expertinnen-Datenbank eine Konzentration auf Wien: $60 \%$ sind in der Hauptstadt tätig. Daneben sind lediglich die Steiermark (17\%) und mit Abstrichen Niederösterreich (9 \%) in nennenswertem Umfang vertreten. 
Tabelle 21: Einträge Expertinnen-Datenbank

Anteil

\begin{tabular}{lc}
\hline Einträge gesamt (Basis) & $\mathbf{1 2 2 0}$ \\
\hline Nach Region & \\
Österreich & $93 \%$ \\
\hline darunter: & $60 \%$ \\
Wien & $17 \%$ \\
Steiermark & $9 \%$ \\
Niederösterreich & $5 \%$ \\
Oberösterreich & $3 \%$ \\
Tirol & $3 \%$ \\
Kärnten & $2 \%$ \\
Salzburg & $0 \%$ \\
Vorarlberg & $0 \%$ \\
Burgenland & $0 \%$ \\
\hline Keine Angabe & $7 \%$ \\
\hline Andere Länder & \\
\hline Nach derzeitiger Tätigkeit & $36 \%$ \\
\hline Universität/Fachhochschule & $21 \%$ \\
\hline Außeruniversitäre Forschungseinrichtung & $20 \%$ \\
\hline Wirtschaft/Industrie & $14 \%$ \\
\hline Freiberuflich & $3 \%$ \\
\hline Karenz/Wiedereinsteigerin & $1 \%$ \\
\hline Berufseinsteigerin & $16 \%$ \\
\hline Sonstige &
\end{tabular}

Quellenangabe: Basis: Datenbank-Dump Dezember 2010

Dass die Expertinnen-Datenbank in erster Linie durch die registrierten Frauen selbst sowie durch die engere FEMtech Community genutzt wird, jedoch kaum in der Breite der FTI-Landschaft, wurde in der Online-Befragung (vgl. Kapitel 3.2.2 „Reichweite und Nutzung insgesamt") deutlich. Insofern erfüllt sie in erster Linie eher eine Vernetzungsfunktion für die Expertinnen und enger an das Programm gebundene Zielgruppen, als dass sie in der Breite ein Recherchetool darstellt. Betrachtet man zudem genauer die Personen, welche die Expertinnen-Datenbank laut Online-Befragung als RechercheurInnen nutzen, zeigt sich, dass viele aus dem universitären Bereich stammen, wenige hingegen aus privatwirtschaftlichen Unternehmen.

Der bereits erwähnte Überhang an eingetragenen Expertinnen und RechercheurInnen aus dem universitären Bereich zeigt sich bei den ausgezeichneten Expertinnen des Monats in noch deutlicherem Maße (49\%). Angesichts der öffentlichen Wahrnehmung dieser Maßnahme ist davon auszugehen, dass die häufige Porträtierung erfolgreicher Frauen an Hochschulen mit dazu beiträgt, dass privatwirtschaftliche Unternehmen sich insgesamt weniger als Zielgruppe von FEMtech angesprochen fühlen. In zahlreichen offenen Anmerkungen von 
UnternehmensvertreterInnen in der Online-Befragung wird deutlich, dass diese FEMtech in erster Linie als Programm für Wissenschaft und Forschung wahrnehmen und deshalb für sich persönlich und beruflich als nicht relevant erleben.

Ein differenzierteres Bild vermitteln die prämierten Fachfrauen bezogen auf ihren Arbeitsort, da im Gegensatz zu der Gesamtheit aller Datenbank-Einträge der größere Teil der ausgezeichneten Frauen nicht in Wien beschäftigt ist. Expertin des Monats ist damit eine der wenigen FEMtech Aktivitäten, welche in wahrnehmbarem Maße auch Wissenschaft und Forschung in den Regionen abbilden.

Tabelle 22: Expertinnen des Monats

\begin{tabular}{lc} 
Expertinnen gesamt (Basis) & $\begin{array}{c}\text { Anzahl } \\
\text { Anteil }\end{array}$ \\
\hline Region & $\mathbf{6 9}$ \\
Österreich & \\
\hline darunter: & $93 \%$ \\
Wien & $41 \%$ \\
Steiermark & $20 \%$ \\
Niederösterreich & $9 \%$ \\
Oberösterreich & $10 \%$ \\
Tirol & $6 \%$ \\
Kärnten & $1 \%$ \\
Salzburg & $4 \%$ \\
Vorarlberg & $1 \%$ \\
\hline Andere Länder & $7 \%$ \\
\hline Derzeitige Tätigkeit & \\
Universität/Fachhochschule & $49 \%$ \\
\hline Wirtschaft/Industrie & $33 \%$ \\
\hline Außeruniversitäre Forschungseinrichtung & $25 \%$ \\
\hline Freiberuflich & $4 \%$ \\
\hline Sonstige & $6 \%$ \\
\hline
\end{tabular}

Quellenangabe: Basis: Liste FEMtech Expertinnen des Monats 2005 bis 2010; Datenbank-Dump Dezember 2010

\subsubsection{Zufriedenheit der Zielgruppen}

Zunächst lässt sich aus den Ergebnissen der Online-Befragung über alle NutzerInnen hinweg eine allgemein hohe Zufriedenheit mit den genutzten FEMtech Aktivitäten konstatieren (die Gesamt-Mittelwerte über alle NutzerInnen hinweg liegen zwischen 1,9 bei Auszeichnungen und 2,5 bei der ExpertinnenDatenbank). Gerade die stark genutzten Angebote wie die Netzwerktreffen oder auch die zentralen Programmorgane Newsletter und Website werden gut bis sehr gut bewertet. Die im Vergleich kritischere Bewertung der Expertin- 
nen-Datenbank relativiert sich, wenn man zwischen den verschiedenen Nutzungsarten unterscheidet: Expertinnen, die lediglich registriert sind, aber die Datenbank noch nie zu Recherchezwecken genutzt haben, sind unzufriedener (Mw. 2,8) als Personen, die nur recherchiert haben (Mw. 2,3). Nutzerinnen, die sowohl Expertin als auch Rechercheurinnen sind, werten hingegen am besten (Mw. 2,1). Die schlechtere Wertung der registrierten Expertinnen ohne Rechercheerfahrung mag mit der geringen Anfragequote von $12 \%$ (siehe oben, vgl. Kapitel 3.2.3 „Nutzung und erreichte Zielgruppen je Aktivität“) zusammenhängen. Offensichtlich wurden ihre Erwartungen in Bezug auf die Datenbank nicht voll erfüllt.

Eine Differenzierung der Zufriedenheit nach Unternehmen, Universitäten und Forschungseinrichtungen offenbart allerdings z. T. deutliche Wertungsunterschiede. Unternehmen beurteilen durchgängig fast alle Angebote - vor allem aber Forum NaWi)(Tech und die Expertinnen-Datenbank - deutlich kritischer. Gerade im Falle des Forum NaWi)(Tech ist dies wohl ein deutlicher Hinweis darauf, dass der erhoffte Nutzen ausblieb. Da sich dieses Format als Recruiting-Veranstaltung dezidiert an Unternehmen mit Bedarf im Bereich technisch-naturwissenschaftlichen Nachwuchses richtet, wurden die diesbezüglichen Erwartungen der Unternehmen offensichtlich nicht ausreichend erfüllt.

Abbildung 25: Zufriedenheit mit FEMtech Aktivitäten - nach FTIOrganisationstyp

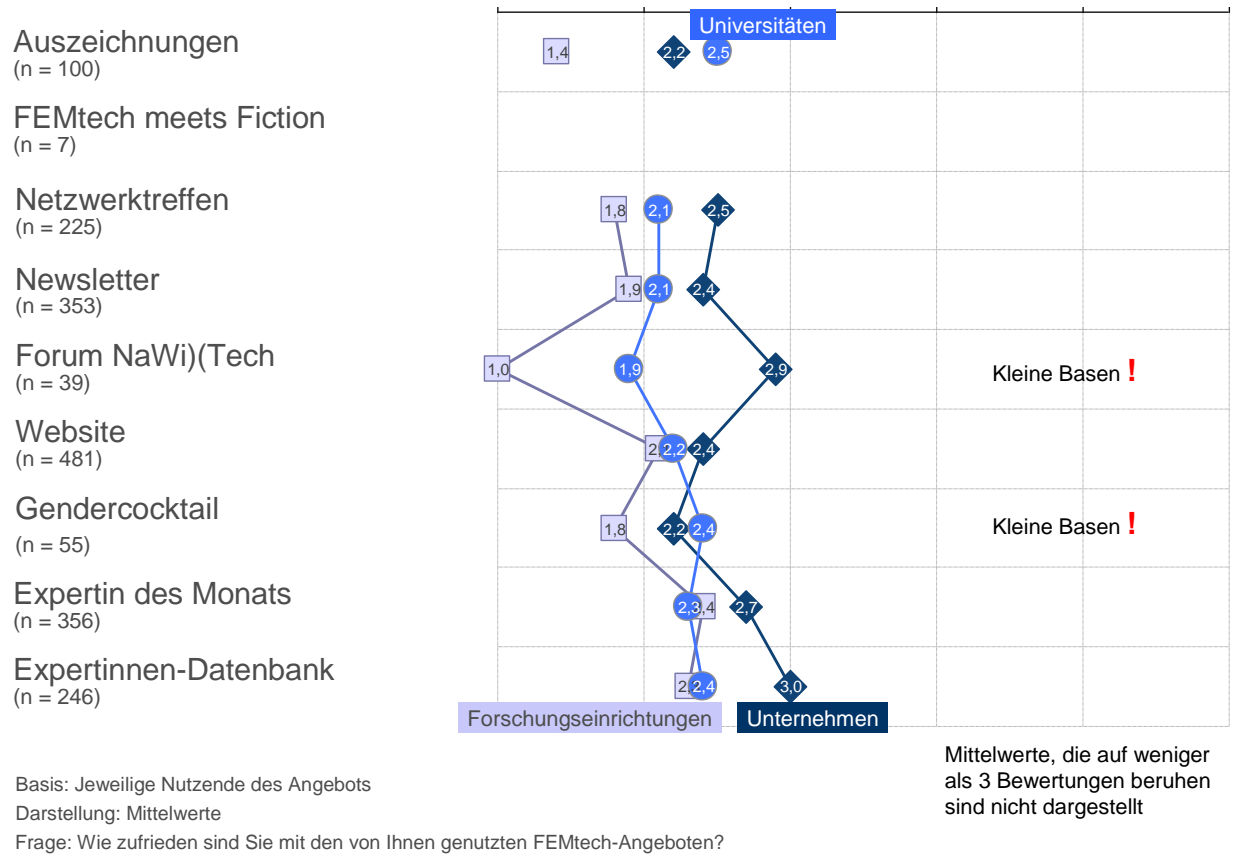

Quellenangabe: ARGE „3C“

\subsubsection{Verbesserungsvorschläge aus Zielgruppensicht}

Anmerkungen und Verbesserungsvorschläge der Zielgruppen gehen zumeist in Richtung eines Ausbaus bzw. der Erweiterung von Maßnahmen, die man prinzipiell als nützlich erachtet. 
Ein in der Online-Befragung häufig geäußerter Verbesserungsvorschlag zielt auf eine stärkere Beachtung der Regionalität bei den Netzwerktreffen: Gut die Hälfte aller Nicht-WienerInnen (17 von insgesamt 33), die schon auf Netzwerktreffen waren und auf die offene Frage nach Verbesserungsvorschlägen zu von ihnen genutzten FEMtech Instrumenten antworteten, äußern diesen Wunsch. Auch FTI-politische AkteurInnen betonen in den Interviews die Notwendigkeit, in allen Bundesländern adäquat vertreten zu sein und die Möglichkeit zu nutzen, dort an vorhandene Strukturen anzudocken und FEMtech damit längerfristiger in den Regionen zu verankern. Durch ihren bereits auf Austausch und Verständigung abzielenden Charakter werden die Netzwerktreffen zudem als geeignetes Vehikel gesehen, um einen bereits hoch sensibilisierten Kreis regelmäßig Besuchender um Gruppen zu erweitern, die mit den Themen Chancengleichheit und Gender bisher kaum erreicht wurden, aber für den Prozess eines gesellschaftlichen „Mainstreamings“ unerlässlich sind. Dazu gehören personalstarke männlich dominierte Berufsfelder genauso wie privatwirtschaftliche Unternehmen, die auf den Treffen immer noch unterrepräsentiert sind.

Der Expertinnen-Datenbank wird im Tandem mit der regelmäßigen Auszeichnung einer Expertin des Monats eine stärkere Bewerbung und damit Sichtbarmachung der registrierten Expertinnen empfohlen (knapp $10 \%$ von 99 Befragten, die mindestens eines der beiden Instrumente kennen und sich zu der offenen Frage nach Verbesserungsvorschlägen äußern, regen dies an). Dies könnte z. B. auch über eine Verknüpfung mit anderen Datenbanken und Portalen (z. B. XING) erfolgen. Andere Äußerungen (ebenfalls knapp $10 \%$ der benannten Teilgruppe) zielen eher auf einen Relaunch ab, um die Datenbank zu aktualisieren, die Funktionalität der Recherchefunktion zu verbessern (schneller, andere Kategorien) und mit einer genaueren Beschreibung der Forschungsgebiete zu versehen. In Bezug auf die Expertinnen des Monats wird von einer Stimme die mediengerechtere Aufbereitung der Porträts angeregt.

Der FEMtech Website raten die Zielgruppen eine stärkere Fokussierung (2 von 115, die die Website nutzen und sich zur offenen Frage nach Verbesserungsvorschlägen für durch sie genutzte FEMtech Instrumente äußern). Angesichts der Fülle zur Verfügung stehender Informationen, Daten, Publikationen und Hinweisen leide die Übersichtlichkeit, wie auch Teilnehmende der On-SiteBefragung zur Website anmerken (drei von insgesamt sechs Befragten, die sich zu dieser offenen Frage äußern, regen dies an - für genügend involvierte Zielgruppen, die sich also die Mühe machen, spontane Verbesserungsvorschläge zu formulieren, offensichtlich ein Anliegen!). So brauche man etwa zu lange, bis man den Zugang zur Expertinnen-Datenbank entdeckt. Dahinter steckt letztlich auch der Wunsch nach einer zielgruppenorientierten Ansprache über die Startseite.

\subsection{FEMtech Wissen}

Der Programmbereich FEMtech Wissen soll dazu dienen, die Kenntnisse zum Thema Frauen in Forschung und Technologie zu erhöhen. Zu diesem Zweck hat FEMtech es sich zum Ziel gesetzt, zentrale Informationen in diesem Themenfeld aufzubereiten und leicht zugänglich zu machen. FEMtech Wissen ver- 
steht sich als „Sammelpunkt für sämtliche Daten und Fakten, Studien und Publikationen aus dem Bereich Frauen in Forschung und Technologie" (FEMtech (o.J.c)). Zentrales Medium für diesen Programmbereich ist die Website. Hier werden die einzelnen Informationsangebote unter dem Titel „Daten“, „genderDiskurs“, „FEMtech Publikationen“ und „Literatur“ bereitgestellt. Darüber hinaus werden ausgewählte Publikationen auch gezielt verschickt. Unter dem Titel „Themen“ bietet FEMtech seit Dezember 2010 außerdem eine strukturierte Aufbereitung der Themen „Wissenschaftlicher Nachwuchs \& Nachwuchsförderung“, „Mentoring in F\&E“ und „Gender Budgeting in der Forschung(sförderung)“. Hierzu wird zunächst das jeweilige Thema kurz beschrieben, dann folgen Beispiele aus der Praxis und schließlich Literaturhinweise bzw. -links.

Im Folgenden werden zunächst die verschiedenen Angebote strukturiert, beschrieben und analysiert. In einem zweiten Schritt werden die Ergebnisse aus der Online-Befragung zur Nutzung, zur Zufriedenheit der erreichten Zielgruppe und zu den Verbesserungsvorschlägen der Zielgruppe präsentiert.

\subsubsection{Inhaltliche Kurzbeschreibung und Analyse}

\section{Monitoring (Daten)}

FEMtech präsentiert unter dem Titel „Daten“ sowohl aufbereitete Tabellen und Grafiken zur geschlechtsspezifischen Beschäftigung im Bereich Forschung, Technologie und Entwicklung in Österreich als auch schriftliche Auswertungen bzw. Zusammenfassungen dieser Daten. Diese Informationen sind zunächst für die wissenschaftlich Beschäftigten in Forschung \& Entwicklung insgesamt dargestellt. Des Weiteren wird nach Art der Einrichtung differenziert zwischen außeruniversitärer Forschung, Universitäten, Hochschulen und Höheren Schulen. Die Daten zu den Beschäftigten in Forschung \& Entwicklung sind sowohl nach Durchführungssektoren (Hochschulsektor, privater gemeinnütziger Sektor, Sektor Staat, Unternehmenssektor) als auch nach Unternehmenssektoren getrennt aufbereitet. Diese Zweiteilung des Datenmaterials von Forschung \& Entwicklung einerseits und den einzelnen Einrichtungen andererseits erschließt sich nicht auf den ersten Blick und erschwert insbesondere den $\mathrm{Zu}$ gang zu den Unternehmens-Daten, da die Unternehmen als Einrichtung nicht in den Unterkategorien enthalten sind, obwohl sie eine der Hauptzielgruppen von FEMtech sind.

Die Daten stammen aus der F\&E-Erhebung der Statistik Austria, der Gender Booklet-Erhebung, der Schulstatistik sowie dem Datawarehouse Hochschulbereich des Bundesministeriums für Wissenschaft und Forschung (BMWF). Die unterschiedlichen Quellen und das Zeitintervall ihrer Erhebung sind sehr detailliert beschrieben. FEMtech erläutert jedoch nicht, wie das unterschiedliche Datenmaterial für die einzelnen Tabellen zusammengefasst wurde. Insbesondere stellt sich die Frage, wie die eigenen Erhebungen, die im Gender Booklet veröffentlicht werden (siehe unten), mit den offiziellen Statistiken von Statistik Austria zusammengefügt werden.

Insgesamt werden 63 Tabellen bzw. Grafiken präsentiert, wobei der Schwerpunkt bei den Unternehmen und den Universitäten liegt. Die Daten werden in 
drei verschiedenen Dateiformaten zur Verfügung gestellt. Bei den Tabellen und Grafiken ist die Bildqualität nicht sehr gut, da viele Informationen auf einer PDF-Seite aufgeführt werden und die Inhalte teilweise nur durch ein Vergrößern lesbar sind.

Auf jeder Unterseite (Forschung \& Entwicklung, Außeruniversitäre Forschung, Universitäten, Fachhochschulen, Höhere Schulen) wird zunächst die Methode der Datenzusammenstellung und -aufbereitung beschrieben, gefolgt von einer kurzen Analyse. Bei dieser Auswertung werden teilweise keine Jahreszahlen als Referenz genannt, sodass nicht erkennbar ist, auf welches Jahr bzw. welchen Zeitraum sich die Analyse bezieht. Da die Texte auch nicht zeitlich gekennzeichnet sind (z. B. „Stand 2010“), fehlt teilweise der Kontext zu den genannten Zahlen.

\section{FEMtech Publikationen}

Die Website enthält unter den Navigationspunkten „genderDiskurs“ und „FEMtech Publikationen“ insgesamt 37 FEMtech Publikationen, die nach ihrem Erscheinungsdatum chronologisch aufgelistet sind. Jede Publikation wird mit einem Titel und ggf. Untertitel, Bild des Covers, einer kurzen Beschreibung und einem Link zum Download vorgestellt. Obwohl es Reihen und Themenschwerpunkte gibt, sind die FEMtech Publikationen nicht thematisch sortiert. Es gibt außerdem für den Bereich „FEMtech Publikationen“ keinen Einleitungstext, der Informationen darüber bietet, nach welchem Konzept FEMtech veröffentlicht und welches Themenspektrum abgedeckt wird.

Die Veröffentlichungen können nach Art der Publikation in drei Gruppen unterteilt werden:

- Studien und Ausarbeitungen

- Dokumentationen von FEMtech Maßnahmen

- Praxisorientierte Publikationen

\section{Studien und Ausarbeitungen}

Die bekannteste FEMtech Publikation, das Gender Booklet, erscheint jährlich seit 2003. Es enthält in erster Linie die Ergebnisse der Erhebung von geschlechtsdifferenzierten Daten zur Beschäftigungssituation in der außeruniversitären naturwissenschaftlich-technischen Forschung. Diese Daten werden auf der Basis von quantitativen und qualitativen Befragungen von 30 bis 80 Einrichtungen gewonnen. 2003 wurden lediglich 31 Einrichtungen befragt, 2008 waren es 56. Die höchste Beteiligung war 2004 mit 93 Einrichtungen. Der Umfang der Publikation ist von ursprünglich 27 Seiten auf mittlerweile 61 Seiten angestiegen.

Während von 2003 bis 2007 eine merkmalsbezogene Erhebung zu bestimmten Kategorien wie Beschäftigungsart, Alter, Fachbereich, Einkommen und Funktion durchgeführt wurde, hat 2008 eine Umstellung stattgefunden auf eine personenbezogene Erhebungsmethode. „Sie ermöglicht die Erstellung eines anonymisierten Individualdatensatzes, der die Verknüpfung unterschiedlicher Merkmale miteinander erlaubt. Eine stärker analytische Ausrichtung des Gender Booklets und eine Anknüpfung an gegenwärtige gesellschaftspolitische Dis- 
kussionen, wie bspw. über geschlechtsspezifische Einkommensunterschiede oder über Frauen in Führungspositionen, werden so möglich. Aus wissenschaftlicher Sicht bietet dieses Vorgehen den Vorteil, nun auch differenziertere Auswertungen mit Hilfe komplexer statistischer Methoden und Verfahren vornehmen zu können."(Gender Booklet 2008)

Seit 2006 gibt es zusätzlich zu den Auswertungen der Befragung ein Schwerpunktthema wie z. B. die Karenzzeit für Väter. Die Struktur des Gender Booklets hat sich seit 2003 stark verändert, neue Themen und Erhebungen sind dazugekommen. FEMtech erhebt den Anspruch, dass das Gender Booklet vergleichbare und präzise Daten zur Situation von Frauen in den Einrichtungen liefert. Die Zahl der befragten Einrichtungen und die Zahl der teilnehmenden MitarbeiterInnen pro Einrichtung werden jedoch nicht an zentraler Stelle, bzw. teilweise gar nicht genannt. Problematisch ist auch, dass die Auswertungen im Gender Booklet als „Langzeitstudie“ bezeichnet werden, obwohl die Anzahl der untersuchten Einrichtungen nicht gleichbleibend ist. Zwischen 2004 und 2006 haben sich 80-93 Einrichtungen an den Befragungen beteiligt, im Jahr 2008 aber nur noch 56.

Die Publikation BRENNPUNKT erscheint seit 2010 in der neuen Reihe „Innovationsfaktor Humanressourcen" von BMVIT/FEMtech und Forschung Austria, die sich mit aktuellen Themen auf dem Gebiet der Humanressourcenpolitik in der außeruniversitären naturwissenschaftlich-technischen Forschung befasst. Besonderen Stellenwert nehmen die Themen Gender Mainstreaming und Chancengleichheit ein.

Im Rahmen dieser neuen Reihe sollen sich in Zukunft die Publikationen BRENNPUNKT (Zusammenfassungen der Ergebnisse fokussierter, einmaliger Studien zu aktuellen Fragestellungen wie der Karriereförderung des Forschungsnachwuchses) und Gender Booklet (Langzeituntersuchung zur Situation von Frauen und Männern in der außeruniversitären naturwissenschaftlich-technischen Forschung) im Jahresrhythmus abwechseln. Das erste Heft ist im November 2010 online gestellt worden und enthält die Ergebnisse einer Studie, die im Auftrag von FEMtech und Forschung Austria mit Nachwuchswissenschafterinnen durchgeführt ${ }^{16}$ wurde, sowie Fallbeispiele von außeruniversitären Forschungseinrichtungen in Österreich und Deutschland.

In der Reihe genderDiskurs werden seit 2009 Beiträge zu aktuellen Themen im Bereich „Frauen in Forschung und Technologie“ veröffentlicht. genderDiskurs erscheint viermal jährlich und soll nach Angaben von FEMtech in jeder Ausgabe ein Schwerpunktthema behandeln, wie z. B. die aktuelle Beschäftigungssituation in Forschung und Entwicklung (F\&E), die Möglichkeiten, naturwissenschaftlich-technische Berufe für Frauen attraktiver zu machen, oder die Situation von Frauen in bestimmten Berufsfeldern. Der Umfang von genderDiskurs liegt bei 6-12 Seiten. Teilweise werden nicht konkrete Themen vorgestellt, sondern Projekte aus den Förderlinien von FEMtech bzw. Analysen der Entwicklung der F\&E-Beschäftigung. So wurde im genderDiskurs 04 über FEMtech FTI-Projekte berichtet und im genderDiskurs 05 über FEMtech Karriere. Der genderDiskurs 06 enthält ähnlich wie das Gender Booklet eine

16 Für die Studie sind 1.214 Nachwuchsforscherinnen mittels Online-Fragebogens kontaktiert worden, der Rücklauf betrug $35 \%$. 
Datenanalyse der Beschäftigungssituation in F\&E. Schwerpunktthemen werden in einen europäischen oder vereinzelt in einen internationalen Kontext gesetzt. Die Reihe "genderDiskurs“ wird auf der Homepage als einzige FEMtech Publikation gesondert mit einem eigenen Reiter dargestellt.

Weitere Studien/Ausarbeitungen von FEMtech sind:

- Erhöhung der Präsenz von Wissenschafterinnen in technischen Entwicklungsprojekten und Programmen (2001)

- Betriebliche Frauenförderung (2004)

- Der Frauenanteil in BMVIT-Programmen und Maßnahmen zur Erhöhung der Chancengleichheit in künftigen Ausschreibungen (2004)

- Kosten-Nutzen-Indikator von gendersensiblen Maßnahmen auf betrieblicher Ebene (2005)

Seit 2005 sind außer den regelmäßigen Erhebungen für die Erstellung des Gender Booklets erst wieder 2009 eine Gender-Budgeting-Studie mit dem Titel "Gender Budgeting in Forschungs- und Technologieprogrammen" sowie 2010 eine Studie mit dem Titel „Einkommensunterschiede zwischen Frauen und Männern im Bereich Forschung und Entwicklung" durchgeführt worden. Bei den eigenen Studien und Ausarbeitungen ist ein Schwerpunkt auf Monitoring und Datenerhebung zu verzeichnen. Die Reihe genderDiskurs wird über die Website als FEMtech Publikation sehr hervorgehoben, wird aber ihrem Anspruch, aktuelle Themen zu „Frauen in Forschung \& Technologie“ zu behandeln, nur teilweise gerecht. Dafür ist einerseits das Themenspektrum zu einseitig und andererseits die Anzahl der Veröffentlichungen über FEMtech Maßnahmen zu hoch. Die ausgewerteten Ausgaben der Reihe genderDiskurs 01-09 behandeln überwiegend Aspekte der Beschäftigungssituation von Frauen und Männern in Forschung und Entwicklung sowie Aktivitäten und Maßnahmen zur Erhöhung der Chancengleichheit. Themen wie wissenschaftliche Nachwuchsförderung und Work-Life-Balance sind noch nicht thematisiert worden.

\section{Dokumentation von FEMtech Maßnahmen}

Insgesamt 13 Publikationen beschreiben FEMtech Maßnahmen aus den anderen Programmbereichen. In dem 2009 erschienenen Band „Staatspreis für Chancengleichheit in F\&E“ werden die GewinnerInnen und die nominierten Einrichtungen für diese Maßnahme aus dem Bereich FEMtech Aktivitäten dargestellt. Seit 2005 werden jährlich die FEMtech „Expertinnen des Monats“ vorgestellt. ${ }^{17}$ Die Publikation hat sich bis 2009 im Umfang fast verdoppelt von 35 auf ca. 60 Seiten. 2006 lag ein Schwerpunkt auf der Situation von Frauen in F\&E in Europa, dieser Band wurde auch ins Englische übersetzt. In der Ausgabe von 2009 sind nicht nur die Expertinnen vorgestellt worden, sondern auch Berufsinformationen aus dem Bereich F\&E. Außerdem werden in zwei Ausgaben der Reihe „genderDiskurs“ (04 und 05) die Förderlinien FEMtech Karriere und FEMtech FTI-Projekte präsentiert. Zu der Programmlinie FEMtech Karriere sind seit 2009 darüber hinaus drei Publikationen erschienen. Der erste

\footnotetext{
172005 lautete die Bezeichnung noch „Forscherin des Monats“.
} 
Band enthält einen Rückblick auf die letzten fünf Jahre und stellt zwölf Einrichtungen vor, die im Rahmen des Programms Förderungen erhalten haben. Dieser Band im Umfang von ca. 60 Seiten ist auch auf Englisch erhältlich. In einem weiteren Band von 2009 sind auf 13 Seiten unter dem Titel „Good Practice Beispiele" neun von FEMtech geförderte Projekte präsentiert.

\section{Praxisorientierte Publikationen}

Zwei Publikationsreihen lassen sich in einen Bereich praxisorientierter Publikationen (Leitfäden, Handlungs- und Argumentationshilfen) zusammenfassen. Hierzu gehören:

- FEMtech Argumentarium (2006, 2009)

- Handbuch Gender in den Forschungs- und Technologieprogrammen des BMVIT (2005) mit den folgenden Anlagen:

- Hintergrundinformationen zu Gender Mainstreaming als argumentative Unterstützung

- Leitfaden zur sprachlichen Gleichstellung von Frauen und Männern in den FTE-Programmen des BMVIT

- Leitfaden zur gendersensiblen Durchführung von Veranstaltungen und Workshops in den FTE-Programmen des BMVIT

Die Anlagen zum Handbuch werden auf der Website als eigenständige Publikationen dargestellt. Mit diesen Publikationen ebenso wie mit den GoodPractice-Beispielen versucht FEMtech, den NutzerInnen praxisorientierte Unterstützung in der konkreten Umsetzung von Gleichstellungsmaßnahmen bzw. Gender Mainstreaming anzubieten.

\section{Analyse der FEMtech Publikationen}

Zur Verbreitung der FEMtech Publikationen liegen nur wenige Informationen vor. Die Publikation „FEMtech Expertinnen“ ist die einzige FEMtech Publikation, die in einer vergleichsweise hohen Auflage von 2000 Exemplaren gedruckt und verschickt worden ist. Die Mehrzahl der Veröffentlichungen liegt ausschließlich im pdf-Format vor und ist online zugänglich. Aus der Reihe "genderDiskurs“ wurden insgesamt an 3.268 Personen pdf-Versionen der Publikationen verschickt.

Für die Evaluation wurden die in den FEMtech Publikationen behandelten Themen neun Kategorien zugeordnet, um zu analysieren, welche Themen bei FEMtech im Mittelpunkt stehen: 
Tabelle 23: Themen der FEMtech Publikationen

\begin{tabular}{|c|c|c|}
\hline & Anzahl & Anteil \\
\hline Gesamt & 37 & $100 \%$ \\
\hline $\begin{array}{l}\text { Attraktivität naturwissenschaftlich-technischer Berufe für Frauen } \\
\text { (Sichtbarmachung, role models, Informationen zu Berufsfeldern) }\end{array}$ & 9 & $24 \%$ \\
\hline $\begin{array}{l}\text { Fördermaßnahmen und Aktivitäten von Unternehmen und außeruni- } \\
\text { versitären Forschungseinrichtungen zur Verbesserung der Chancen- } \\
\text { gleichheit (Good Practice, Mentoring, FEMtech Programmlinien, finan- } \\
\text { zielle Bonussysteme in der Forschungsförderung) }\end{array}$ & 9 & $24 \%$ \\
\hline $\begin{array}{l}\text { Beschäftigungssituation/Chancengleichheit von Frauen \& Männern in } \\
\text { der außeruniversitären F\&E (Gender Pay Gap, Arbeitskultur, Frauen } \\
\text { in Führungspositionen) }\end{array}$ & 6 & $16 \%$ \\
\hline $\begin{array}{l}\text { Gender Mainstreaming (Gender Budgeting, Gender Management, gen- } \\
\text { dersensibles Veranstaltungsdesign, geschlechtergerechte Sprache) }\end{array}$ & 5 & $14 \%$ \\
\hline Work-Life-Balance (Karenzierung und Elternzeit) & 2 & $5 \%$ \\
\hline $\begin{array}{l}\text { Gender in der angewandten Forschung, in FTE-Programmen des } \\
\text { BMVIT }\end{array}$ & 2 & $5 \%$ \\
\hline $\begin{array}{l}\text { Genderspezifisches Monitoring u. Datenanalyse (Honigtopfindikator, } \\
\text { leaky pipeline, EU-Vergleich) }\end{array}$ & 2 & $5 \%$ \\
\hline Wissenschaftlicher Nachwuchs an Universitäten und Fachhochschulen & 1 & $3 \%$ \\
\hline $\begin{array}{l}\text { Betriebswirtschaftliche Gründe für Chancengleichheit, GM und Gleich- } \\
\text { stellungsmaßnahmen }\end{array}$ & 1 & $3 \%$ \\
\hline
\end{tabular}

Quellenangabe: Basis: 18 Studien und Ausarbeitungen, 13 FEMtech Maßnahmen, 6 praxisorientierte Publikationen

Die Verteilung der Themen bei den FEMtech eigenen Publikationen ist breit gefächert und spiegelt teilweise die von FEMtech verfolgten Ziele wider.

Mit jeweils 24,3\% sind die Themen „Attraktivität naturwissenschaftlichtechnischer Berufe für Frauen“ und „Fördermaßnahmen und Aktivitäten zur Verbesserung der Chancengleichheit" am stärksten vertreten. Dies entspricht dem Fokus von FEMtech auf Bewusstseinsbildung und Sensibilisierung sowie auf die Förderung von Chancengleichheit. Hier ist positiv hervorzuheben, dass eine Vielzahl der FEMtech Publikationen darauf ausgerichtet ist, positive Beispiele von Forscherinnen oder Unternehmen zu geben, um die Sichtbarmachung sowohl von Frauen als auch von „Good Practice“-Beispielen zu fördern. Zur Beschäftigungssituation von Frauen und Männern hat FEMtech vergleichsweise wenig publiziert, obwohl die Verbesserung der Rahmenbedingungen, der Situation und der Zugangs- und Karrierechancen von Frauen in F\&E das zentrale Handlungsziel von FEMtech ist.

Die Gewinnung von Nachwuchswissenschafterinnen für Karrieren in F\&E stellt FEMtech als wichtiges Ziel heraus. Mit FEMtech Karrierewege gibt es zu diesem Thema eine eigene Förderlinie, gleichwohl liegt im Bereich der FEMtech Publikationen nur eine Veröffentlichung vor (BRENNPUNKT 2010). Publikationen zu Gender Mainstreaming sind hingegen mit 13,5\% stark vertreten.

Weiters wurden die Veröffentlichungen nach der Art der Publikation (Studien/Ausarbeitungen, Dokumentation von FEMtech Maßnahmen und praxisorientierte Publikationen) unterteilt, um zu untersuchen, wie die verschiedenen Publikationsarten anteilig vertreten sind. 


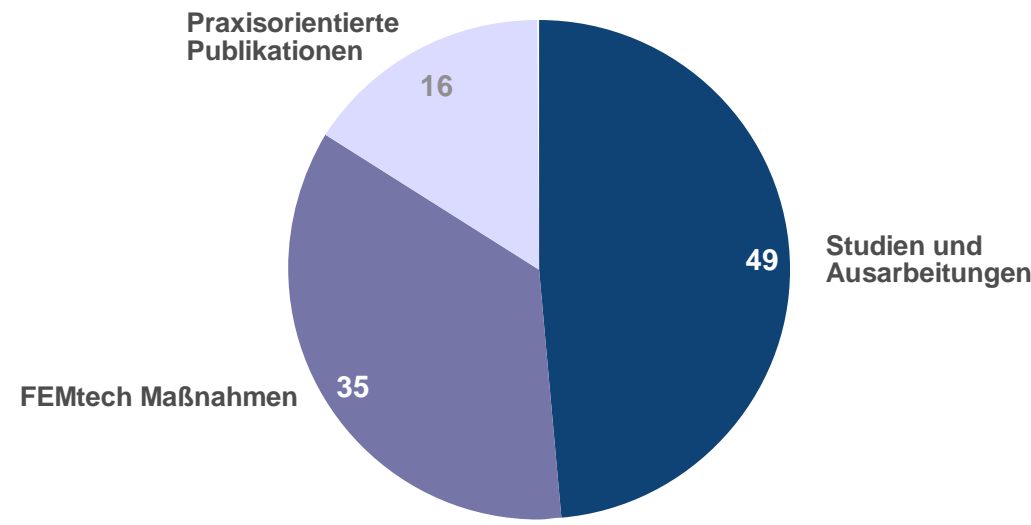

Basis: FEMtech-Publikationen, $\mathrm{n}=37$

Darstellung: Prozentwerte

Quellenangabe: ARGE „3C“

Die Analyse zeigt, dass der Schwerpunkt der 37 Publikationen auf Studien und Erhebungen sowie auf der Dokumentation von FEMtech Maßnahmen liegt. Der Anteil der praxisorientierten Publikationen ist im Verhältnis gering.

Welche Zielgruppe mit den Produkten im Bereich Wissen angesprochen werden soll, geht weder aus den Programmdokumenten noch aus der Beschreibung auf der Website hervor. Daher kann auch kein Abgleich zwischen anvisierter und erreichter Zielgruppe erfolgen. Die Portfolioanalyse zeigt jedoch, dass eine der Hauptzielgruppen von FEMtech, die Unternehmen, weder über die Angebote direkt angesprochen wird (vgl. Aufbau des Datenmaterials) noch Gegenstand der Publikationen und regelmäßigen Erhebungen ist. Das Gender Booklet untersucht z. B. nur Einrichtungen in der außeruniversitären Forschung. Auch die Expertinnen des Monats, die jährlich in einer Broschüre vorgestellt werden, kommen überwiegend nicht aus dem Unternehmensbereich. So sind von den zwölf Expertinnen des Monats im Jahr 2009 nur vier in Unternehmen tätig.

\section{Externe Publikationen/Literaturhinweise}

Der dritte und umfangreichste Bereich im Rahmen von „Wissen“ sind die Download-Links sowie Literaturhinweise zu internen und externen Publikationen. Auf der FEMtech Website befinden sich unter dem Titel „Literatur“ mehr als 300 Einträge zu nationalen und internationalen, internen und externen Publikationen. Die Publikationshinweise sind nach zwölf Themen sortiert (Außeruniversitäre \& industrielle Forschung, Frauen in F\&E, Universitäten, Unternehmen, Innovation, Karrieren in F\&E, Vereinbarkeit, Berufsbilder, Frauen im Film, Gender \& Geld, Gender, Arbeitsmarkt und Mentoring).

Da die Themen sich teilweise überschneiden und Publikationen daher in mehreren Kategorien aufgeführt werden, kann die Anzahl der verfügbaren Publi- 
kationen nicht ohne größeren Aufwand genau bestimmt werden. Innerhalb der einzelnen Themen sind die Publikationen chronologisch abwärts aufgelistet und ebenso wie die eigenen Publikationen enthalten sie Titel, Cover, kurzes Abstract und Download-Link. Es werden sowohl österreichische als auch deutsche und europäische Publikationen aufgelistet. Es gibt keine Erläuterungen zu den Kriterien für die Auswahl der Veröffentlichungen. Auf der Website heißt es hierzu:

„FEMtech ist stets bemüht, das gesammelte Wissen allen Interessierten zur Verfügung zu stellen. In diesem Bereich werden nationale und internationale Studien zum Download bereitgestellt."

Über einen Link „Weiterführende Literatur“ auf den jeweiligen Themenseiten öffnet sich eine Literaturliste, teilweise mit Kurzbeschreibungen der aufgelisteten Publikationen. Die Tatsache, dass es neben der zum Download bereitgestellten Literatur noch weitere Literaturhinweise gibt, wird auf der Website nicht deutlich kommuniziert.

\subsubsection{Nutzung (erreichte Zielgruppen) und Zufriedenheit der Nutz- erlnnen}

Bei den FEMtech Wissensangeboten zeigt sich - ebenso wie im Bereich „Aktivitäten" - eine Bekanntheits- und Nutzungskluft zwischen FEMtech AdressatInnen und FTI-Unternehmen und -Organisationen (FFG). Da im Vergleich zu den FEMtech Aktivitäten die Wissensangebote jedoch ein geringeres Bekanntheitsniveau aufweisen, kann davon ausgegangen werden, dass diese vor allem für einen „inneren Kreis“ mit entsprechenden Forschungsinteressen und Informationsbedürfnissen nützlich sind.

Positiv hervorzuheben ist, dass insbesondere mit dem Gender Booklet der richtige AdressatInnenkreis erreicht wurde. Dies lässt sich aus dem guten Verhältnis von Nutzung und Gesamtreichweite schließen. Kurz gesagt: Wer von den FEMtech Wissensangeboten weiß, nutzt diese häufig auch gezielt. Eine Ausnahme bildet die quartalsweise erscheinende Publikation genderDiskurs, die gegenüber den anderen online verfügbaren Wissensangeboten nur einen kleinen NutzerInnenkreis erreicht.

Die Zufriedenheit mit den FEMtech Wissensangeboten liegt mit vielen Wertungen im 1er-Bereich sogar noch leicht über dem der FEMtech Aktivitäten. Der Anspruch des Kompetenzzentrums, mit FEMtech Wissen einen „Sammelpunkt für sämtliche Daten und Fakten, Studien und Publikationen aus dem Bereich Frauen in Forschung und Technologie“ bereitzustellen, wird also aus Sicht der NutzerInnen durchaus eingelöst.

Im Hinblick auf FTI-Unternehmen und -Organisationen (FFG) allgemein ist diese Zielsetzung jedoch allein schon aufgrund der mangelnden Bekanntheit und damit verbunden der geringen Nutzung nicht erfüllt. 


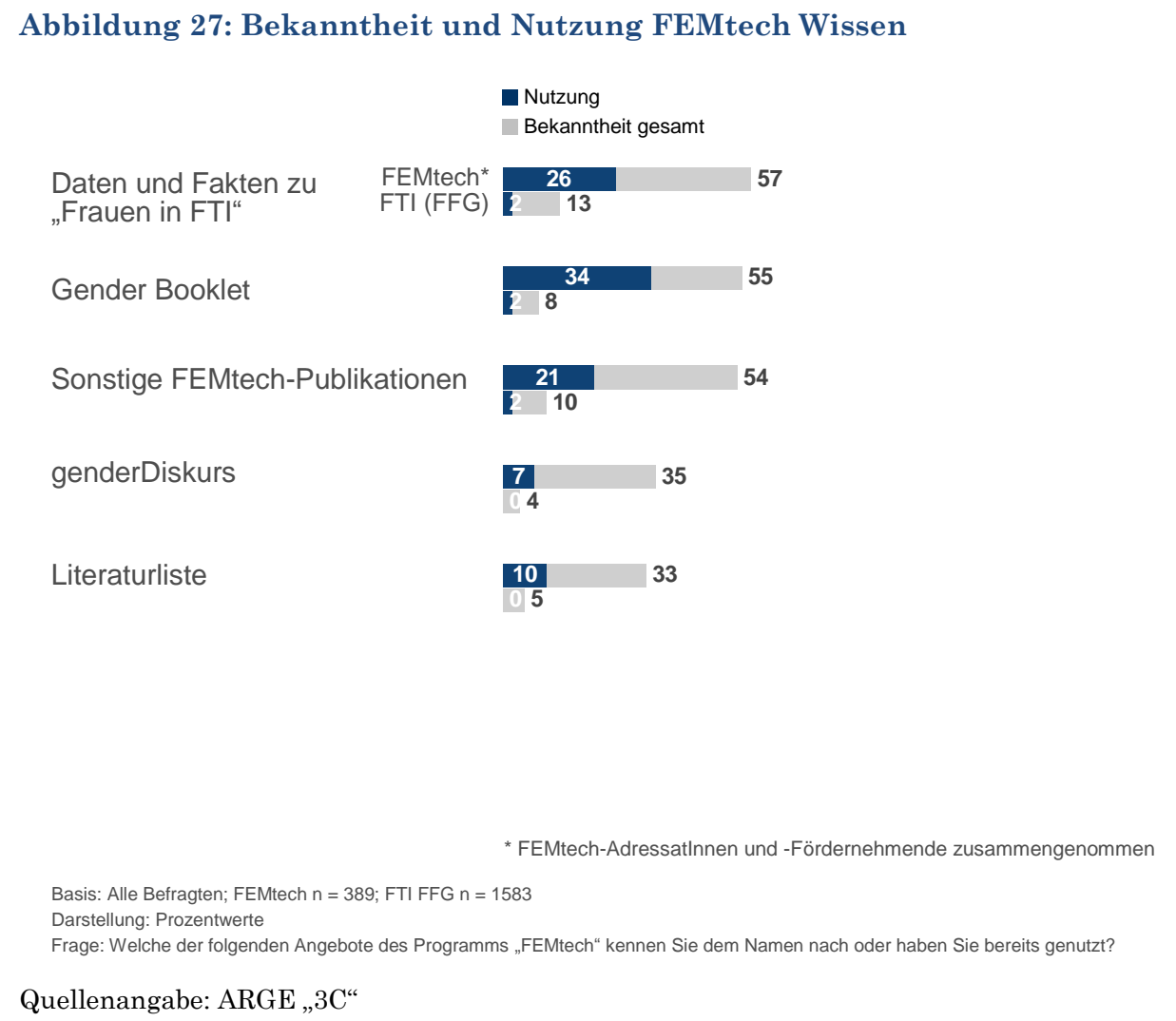

Quellenangabe: ARGE, $3 \mathrm{C}^{\circ}$

Im Vergleich der verschiedenen Anspruchsgruppen zeigt sich aber auch bei den FEMtech Wissensangeboten, dass die bereitgestellten Informationen den Bedürfnissen von Unternehmen in geringerem Maße gerecht werden. So deutet die eher durchschnittliche Bewertung des Bereichs „Daten und Fakten“ darauf hin, dass Unternehmen sie weniger für ihre Zwecke - in vielen Fällen sicherlich die Personalplanung - nutzen (können) als bspw. Forschungseinrichtungen und Universitäten, wo die nach verschiedenen Segmenten und Subsektoren ausdifferenzierten Statistiken insbesondere auch für die sozialwissenschaftliche Forschung interessant sein dürften.

In den Interviews mit den FTI-politischen AkteurInnen und dem oberen Management ist zudem deutlich geworden, dass FEMtech zwar erfolgreich das Thema „Frauen in FTI“ etabliert hat, aber mittlerweile ein gewisser Sättigungsgrad eingetreten ist. FEMtech hat es geschafft, das Thema „Frauen in FTI" mit Fakten zu hinterlegen. Es gibt unterschiedliche Themen, die hier aktiv angegangen werden. Zugleich erscheint es den Befragten so, „als ob Chancengleichheit das Feld in der FTI-Politik ist, in dem es die meisten Erhebungen, Zahlen und Daten gibt" (und eine Sättigung mit Informationen, insbesondere Zahlen und Daten erreicht ist) (Zitat FTI-pA3). Derzeit gibt es genug aufbereitetes Material - vielleicht schon zu viel - jedoch zu wenig Überblick. Das Gender Booklet ist prinzipiell gut, gibt jedoch keine Orientierung für die Forschung (außer man ist ein außeruniversitäres Institut). Es ist dabei nicht interessant, welche kleinen Veränderungen im Personalbereich passieren (,zwei Mitarbeiterinnen gehen, einer kommt - gleich schlechtes Bild") (Zitat FTI-pA2), sondern welche Maßnahmen erfolgreich umgesetzt werden. Hier könnte man mehr lernen. 
Abbildung 28: Zufriedenheit mit FEMtech Wissen - nach FTI-Organisationstyp

$\begin{aligned} & \text { genderDiskurs } \\ & (n=30)\end{aligned}$
$\begin{aligned} & \text { Daten und Fakten zu „Frauen in FTI" } \\ & (\mathrm{n}=131)\end{aligned}$
$\begin{aligned} & \text { Gender Booklet } \\ & (\mathrm{n}=163)\end{aligned}$
$\begin{aligned} & \text { Literaturliste } \\ & (\mathrm{n}=45) \\ & (\mathrm{n}=109)\end{aligned}$

Basis: Jeweilige Nutzende des Angebots

Darstellung: Mittelwerte

Frage: Wie zufrieden sind Sie mit den von Ihnen genutzten FEMtech-Angeboten?

Mittelwerte, die auf wenige als 3 Bewertungen beruhen sind nicht dargestellt

Quellenangabe: ARGE „3C“

\subsubsection{Verbesserungsvorschläge der Zielgruppe}

Im Rahmen der Online-Befragung und in den Interviews sind zum Bereich „Wissen“ nur wenige Verbesserungsvorschläge geäußert worden. Das Angebot von FEMtech an Daten und Fakten wird als positiv hervorgehoben und vorgeschlagen, diese Informationen nicht nur in InsiderInnenkreisen, sondern auch in öffentlichen Medien wie z. B. Tageszeitungen regelmäßig zu vermitteln.

Hinsichtlich der FEMtech Publikationen Gender Booklet und Expertinnen des Monats wurden Zweifel daran geäußert, dass hiermit die „richtigen“ Zielgruppen, nämlich junge Frauen (Schülerinnen und Absolventinnen naturwissenschaftlicher Studiengänge), angesprochen werden.

Zum Gender Booklet wurde in der Online-Befragung bemerkt (2 von 58 Personen, die das Instrument nutzen und sich zur offenen Frage nach Verbesserungsvorschlägen für durch sie genutzte FEMtech Instrumente äußern), dass es bezüglich der Daten informativ sei, der qualitative Teil jedoch eher als „Werbung in eigener Sache“ denn als Forschungsergebnis wahrgenommen werde.

Des Weiteren wird von einer Person darauf hingewiesen, dass es an zielgruppenspezifischen konkreten Beispielen fehle, die auch aus internationaler Perspektive präsentiert werden könnten. 


\subsection{Maßnahmen für breite Öffentlichkeit und technologie- politische Community}

\subsubsection{PR- und Pressearbeit}

Die PR- und Pressearbeit des Kompetenzzentrums versorgt tagesaktuelle und Fachmedien regelmäßig mit Informationen über aktuelle Entwicklungen, laufende FEMtech Maßnahmen sowie Meilensteine. Ein für die Aussendung von Pressemitteilungen, Direktmailings, Einladungen und Programminformationen aufgebauter österreichweiter Verteiler von JournalistInnen und Medien in F\&E umfasst im Juli 2010 rund 300 Kontakte.

Die Anzahl der Pressemitteilungen, OTS-Aussendungen und geschalteten Anzeigen im Zeitverlauf ist ein Beleg für die systematische PR- und Medienarbeit, die mit Gründung des Kompetenzzentrums Anfang 2008 geleistet werden konnte. So kulminieren entsprechende Aktivitäten bereits 2009 in zusammen mehr als 50 ausgesandten Pressemitteilungen und geschalteten Anzeigen.

Tabelle 24: Anzahl Pressemitteilungen und Anzeigenschaltungen

\begin{tabular}{l|c|c|c|c|c|c|c} 
& 2005 & 2006 & 2007 & 2008 & 2009 & 2010 & Gesamt \\
\hline Pressemitteilungen & & 8 & 1 & 12 & 29 & 13 & 63 \\
\hline $\begin{array}{l}\text { APA-OTS-Online- } \\
\text { Aussendungen }\end{array}$ & - & 7 & 1 & 1 & 10 & 3 & 22 \\
\hline Werbeschaltungen & 1 & 11 & 3 & 26 & 25 & 0 & 66 \\
\hline
\end{tabular}

Quellenangabe: Basis: Pressemitteilungen, OTS-Aussendungen und Anzeigenschaltungen April 2005 bis Juni 2010

Darüber hinaus wurden gezielt Kooperationen mit Medien entwickelt, die eine starke F\&E-Affinität aufweisen. Dazu zählen etwa Content-Partnerschaften mit „Factory woman“ (Magazin zu Ausbildung, Beruf und Karrierechancen junger Frauen in der Technik), „INSight“ (Best-Practice-Magazin des Innovationstransferprogramms „Techno Kontakte“) und „AUSTRIA INNOVATIV“ (Fachmagazin für Forschung und Wirtschaft).

\subsubsection{FEMtech Medienresonanz}

In einem Zeitraum von etwas mehr als vier Jahren (04/2006 - 06/2010) erschienen insgesamt mindestens 230 Medienbeiträge zu FEMtech in Print- und Online-Medien, wobei offenbleiben muss, ob es in den früheren Jahren weniger Resonanz gab oder diese nicht systematisch gesammelt wurde. Dabei addieren sich die Printveröffentlichungen, zu denen Auflagenzahlen vorlagen (158 Beiträge), auf eine Gesamt-Auflage von 14.490.888.

Die sichtbar verstärkte Öffentlichkeits- und Medienarbeit zu FEMtech nach Einrichtung des Kompetenzzentrums schlägt sich in einem starken Anstieg der Medienresonanz ab 2009 nieder. So hat sich die Berichterstattung über FEMtech im Laufe eines Jahres von 34 Clippings 2008 auf 113 Clippings 2009 mehr als verdreifacht. Auch für 2010 (Clippings lagen nur für das erste Halbjahr vor) 
kann von einer hohen Medienresonanz ausgegangen werden. In diesem $\mathrm{Zu}$ sammenhang spielen auch die in den letzten Jahren angestoßenen Medienkooperationen eine Rolle, in denen redaktionelle Beiträge veröffentlicht wurden.

Tabelle 25: Medienresonanz

\begin{tabular}{l|c|c|c|c|c|c} 
& 2006 & 2007 & 2008 & 2009 & 2010 & Gesamt \\
\hline Original-Clippings & 3 & 7 & 34 & 113 & 73 & 230 \\
\hline
\end{tabular}

Quellenangabe: Basis: Clippings April 2006 bis Juni 2010

In der weiteren Analyse der Medienresonanz zeigt sich, dass hauptsächlich Zeitungen und Online-Medien erreicht wurden.

Der vergleichsweise ebenfalls hohe Anteil an Beiträgen in Fachzeitschriften geht auch auf Medien des Bohmann-Verlages zurück, zu dem aufgrund seiner Beteiligung am Kompetenzzentrum ein direktes Verhältnis besteht. So entfallen allein auf die Bohmann-Medien „AUSTRIA INNOVATIV“ und „Umweltschutz" zusammengenommen 15 Beiträge. Die geringe Präsenz in Verbandspublikationen hingegen ist letztlich auch Ausdruck der bis dato geringen Vernetzung von FEMtech mit Kammern und Verbänden.

Tabelle 26: Medienresonanz nach Medienart

Anzahl

\begin{tabular}{lc}
\hline Clippings gesamt (Basis) & $\mathbf{2 3 0}$ \\
\hline Medienart & \\
Tageszeitung/Wochenzeitung & $40 \%$ \\
\hline Internet-Publikation & $27 \%$ \\
\hline Fachzeitschrift & $21 \%$ \\
\hline Publikumszeitschrift & $8 \%$ \\
\hline Verbandspublikation (Kammern/Verbände) & $2 \%$ \\
\hline Social Media (Weblog, Twitter) & $1 \%$ \\
\hline
\end{tabular}

Quellenangabe: Basis: Clippings April 2006 bis Juni 2010

Die Erwähnung der verschiedenen FEMtech Maßnahmen in der Medienresonanz lässt darauf schließen, dass FEMtech in erster Linie als Förderprogramm wahrgenommen wird. Dass beinahe die Hälfte dieser Beiträge dabei die konkreten Förderlinien nicht benennt, unterstützt diese Beobachtung zudem, da in diesen Texten FEMtech in Kurzform als „Förderprogramm“ charakterisiert wird.

Ebenfalls häufig genannt wird die „Expertin des Monats“ (37 \% der Beiträge). Dies kann bereits als wichtiger Beitrag zur Wirkung dieser Maßnahme verstanden werden, da sie die publikums- und medienwirksame Präsentation erfolgreicher Frauen in FTI explizit zum Ziel hat. Suboptimal ist allerdings, dass in nur wenigen dieser Beiträge die Expertinnen-Datenbank mitkommuniziert (8\%) wird, aus der die Expertinnen des Monats hervorgehen.

Weitere FEMtech Maßnahmen wie Auszeichnungen (13\%) und Netzwerktreffen (10\%) erhalten eine sporadische mediale Verbreitung im Rahmen von An- 
kündigungen oder Nachberichterstattungen zu konkreten Veranstaltungen. Darüber hinaus wird in der Medienresonanz nur selten auf einzelne Angebote eingegangen.

Tabelle 27: Medienresonanz nach Nennung FEMtech Angebote

\begin{tabular}{lc} 
& $\begin{array}{c}\text { Anzahl } \\
\text { Anteil }\end{array}$ \\
\hline Clippings gesamt (Basis) & $\mathbf{2 3 0}$ \\
\hline FEMtech Förderungen allgemein bzw. unspezifisch & $47 \%$ \\
\hline davon & $10 \%$ \\
FEMtech Karrierewege & $10 \%$ \\
FEMtech FTI-Projekte & $7 \%$ \\
FEMtech Karriere & $37 \%$ \\
\hline FEMtech Website & $35 \%$ \\
\hline Expertin des Monats & $13 \%$ \\
\hline Auszeichnungen & $6 \%$ \\
\hline Savon & $5 \%$ \\
FEMtech meets Fiction & $2 \%$ \\
\hline Gender Award & $10 \%$ \\
\hline FEMtech Netzwerktreffen & $10 \%$ \\
\hline Sonstige FEMtech Publikationen & $8 \%$ \\
\hline Expertinnen-Datenbank & $7 \%$ \\
\hline Gender Booklet & $3 \%$ \\
\hline Daten und Fakten zu „Frauen in FTI & $3 \%$ \\
\hline Forum NaWi)(Tech &
\end{tabular}

Quellenangabe: Basis: Clippings April 2006 bis Juni 2010

Analysiert man die Beiträge nach Themen bzw. danach, welche Aspekte und damit auch Botschaften des Programms transportiert wurden, wird deutlich, dass insbesondere unmittelbare LeserInnenrelevanz und Personalisierung wichtige Kriterien in der Berichterstattung über das Programm darstellen. Fast die Hälfte aller Beiträge (44\%) thematisiert im Kontext der FEMtech Berichterstattung die Attraktivität naturwissenschaftlich-technischer Berufe für Frauen: Etwa, indem sie Informationen zu Berufsfeldern vermitteln und damit für eine jüngere weibliche Leserinnenschaft in der beruflichen Orientierungsphase praktisch relevant sind, oder indem sie erfolgreiche Frauen in FTI (darunter natürlich viele Expertinnen des Monats) sichtbar machen. Hingegen werden konkrete Maßnahmen im Sinne eines Best-Practice (Unterstützung von Frauen beim Berufseinstieg/im Beruf, Verankerung von Gleichstellung, Verbesserung der Work-Life-Balance etc.) nur selten thematisiert (im Bereich $2 \%$ bis $7 \%)$. 
Tabelle 28: Medienresonanz nach Themen

Anzahl

\begin{tabular}{lc}
\hline Clippings gesamt (Basis) & $\mathbf{2 3 0}$ \\
\hline $\begin{array}{l}\text { Attraktivität naturwissenschaftlich-technischer Berufe für Frauen } \\
\text { (Sichtbarmachung, role models, Informationen zu Berufsfeldern) }\end{array}$ & $44 \%$ \\
\hline „Gender“ in Forschungsprojekten & $14 \%$ \\
\hline $\begin{array}{l}\text { Daten und Fakten zur Situation in F\&E (Frauen in Führungsposi- } \\
\text { tionen, Gender Pay Gap etc.) }\end{array}$ & $13 \%$ \\
\hline $\begin{array}{l}\text { Thematisierung Unterstützungsmaßnahmen f. Frauen beim Berufs- } \\
\text { einstieg (Rekrutierung, Kooperationen außeruniversitäre F\&E und }\end{array}$ & $7 \%$ \\
\hline $\begin{array}{l}\text { Hochschulen, Diplomarbeitsbörsen etc.) } \\
\text { Thematisierung Unterstützungsmaßnahmen f. Frauen im Beruf } \\
\text { (Mentoring, Good Practice, Personalentwicklung, Karriereförderung) }\end{array}$ & $5 \%$ \\
\hline $\begin{array}{l}\text { FEMtech Programmvorstellung } \\
\text { Thematisierung Maßnahmen zur Verankerung des Gleichstellungs- } \\
\text { gedankens in Organisationen }\end{array}$ & $5 \%$ \\
\hline $\begin{array}{l}\text { Gender Mainstreaming allg. (Gender Budgeting, Gender Manage- } \\
\text { ment, gendersensibles Veranstaltungsdesign, geschlechtergerechte } \\
\text { Sprache) }\end{array}$ & $4 \%$ \\
\hline $\begin{array}{l}\text { Thematisierung Maßnahmen zur Verbesserung von Vereinbarkeit } \\
\text { von Familie und Beruf und Work-Life-Balance innerhalb von Organi- } \\
\text { sationen (Wiedereinstieg, Karenz, Arbeitszeitflexibilisierung etc.) }\end{array}$ & $2 \%$ \\
\hline $\begin{array}{l}\text { Themenspezifische Vernetzung und Kooperationen von Organisatio- } \\
\text { nen und MultiplikatorInnen }\end{array}$ & $1 \%$ \\
\hline
\end{tabular}

Quellenangabe: Basis: Clippings April 2006 bis Juni 2010

Dass von den AkteurInnen des Programms im Gros der Medienbeiträge zuvorderst das BMVIT als Initiatorin und Trägerin von FEMtech genannt wird, ist kein überraschendes Ergebnis. Erstaunlich ist allerdings, dass in den selbst verantworteten Werbeschaltungen das Kompetenzzentrum nicht konsequenter mitkommuniziert wird (fast alle Werbeschaltungen entfallen auf den Zeitraum nach der Gründung Anfang 2008). Insbesondere Anzeigen im Rahmen von Medienkooperationen (die wie redaktionelle Beiträge gestaltet, aber als Anzeigen erkennbar sind), bewerben oft ausschließlich die FEMtech Förderlinien und nennen in diesem Zusammenhang die FFG als Ansprechpartner.

Die insgesamt hohe Zahl der Werbeschaltungen, in denen FEMtech und BMVIT-Logos zum Teil mittransportiert werden, aber auch der sehr hohe Anteil von Medienbeiträgen, die das BMVIT oder seine VertreterInnen nennen, dürfte dafür verantwortlich sein, dass die interviewten FTI-politischen AkteurInnen FEMtech als sehr stark PR-lastig (,politischer Verkauf für das BMVIT“) wahrnehmen. Sie mahnen ein, dass der Unterschied zwischen AwarenessSchaffung und Politikinstrument hier geschärft werden müsse. 
Tabelle 29: Medienresonanz nach Nennung zentraler AkteurInnen

\begin{tabular}{l|c|c} 
& Clippings & $\begin{array}{c}\text { Werbe- } \\
\text { schaltungen }\end{array}$ \\
& Anzahl & Anzahl \\
\hline Clippings gesamt & $\mathbf{2 3 0}$ & $\mathbf{6 6}$ \\
\hline $\begin{array}{l}\text { Beiträge mit Nennung BMVIT und/oder sei- } \\
\text { ner VertreterInnen }\end{array}$ & $67 \%$ & $89 \%$ \\
\hline $\begin{array}{l}\text { Beiträge mit Nennung FEMtech Kompetenz- } \\
\text { zentrum und/oder seiner VertreterInnen }\end{array}$ & $13 \%$ & $53 \%$ \\
\hline Beiträge mit FEMtech Logo & $3 \%$ & $50 \%$ \\
\hline Beiträge mit BMVIT-Logo & $1 \%$ & $38 \%$ \\
\hline
\end{tabular}

Quellenangabe: Basis: Clippings April 2006 bis Juni 2010

\subsubsection{Agenda-Setting in der FTI-Community}

Das Programm FEMtech verfolgte - ursprünglich auch als eigenen Programmpunkt „Technologieprogramme“, später eher implizit - ein politisches AgendaSetting im Rahmen der Forschungsförderungspolitik. Frauenförderung und Chancengleichheit sollen selbstverständlich werden und in den Mainstream der Forschungsförderung Eingang finden. Daten und (teilweise interne) Studien wurden dazu genutzt. Themen wie Gender Budgeting wurden aufgegriffen, um sie für technologiepolitische AkteurInnen transparenter zu machen. Agenda Setting fand z. T. im „stillen Kreis“ statt, wurde jedoch auch offensiv v. a. durch den „Gendercocktail “ beim Forum Alpbach betrieben. Wurden die politischen AkteurInnen auf diesem Weg erreicht?

Dass es gelungen ist, die Präsenz der Themen Chancengleichheit und Frauenförderung in der FTI-Politik zu stärken bzw. zumindest die politische Aufmerksamkeit darauf zu lenken, zeigt sich in der Onlinebefragung bei den AkteurInnengruppen, welche FTI-Rahmenbedingungen gestalten, FTIAktivitäten fördern oder die Interessen von FTI-Unternehmen und -Organisationen vertreten (Politik/öffentliche Verwaltung, Verbände und Forschungs-/Wirtschaftsförderung). In diesen Gruppen zeigt sich eine hohe Präsenz und breite Akzeptanz der FEMtech Zielsetzungen. Gerade politische AkteurInnen bewerten Chancengleichheit und Frauenförderung als genauso wichtig wie die allgemeine FTI-Nachwuchsgewinnung.

Bei Verbänden offenbart sich allerdings eine leichte Diskrepanz zwischen der Akzeptanz des gleichstellungspolitischen Ansatzes der Chancengleichheit (Schaffung gleicher Chancen für Frauen und Männer führt in vielen Fällen automatisch zu einer Verbesserung der Situation für (Nachwuchs-) Forscherinnen) und dem Ansatz einer gezielten Frauenförderung - auch wenn die $\mathrm{Zu}$ stimmung immer noch eindeutig im positiven Bereich liegt. 
Abbildung 29: Einschätzung der Wichtigkeit von Humanressourcen-Themen durch FTI-politische AkteurInnen

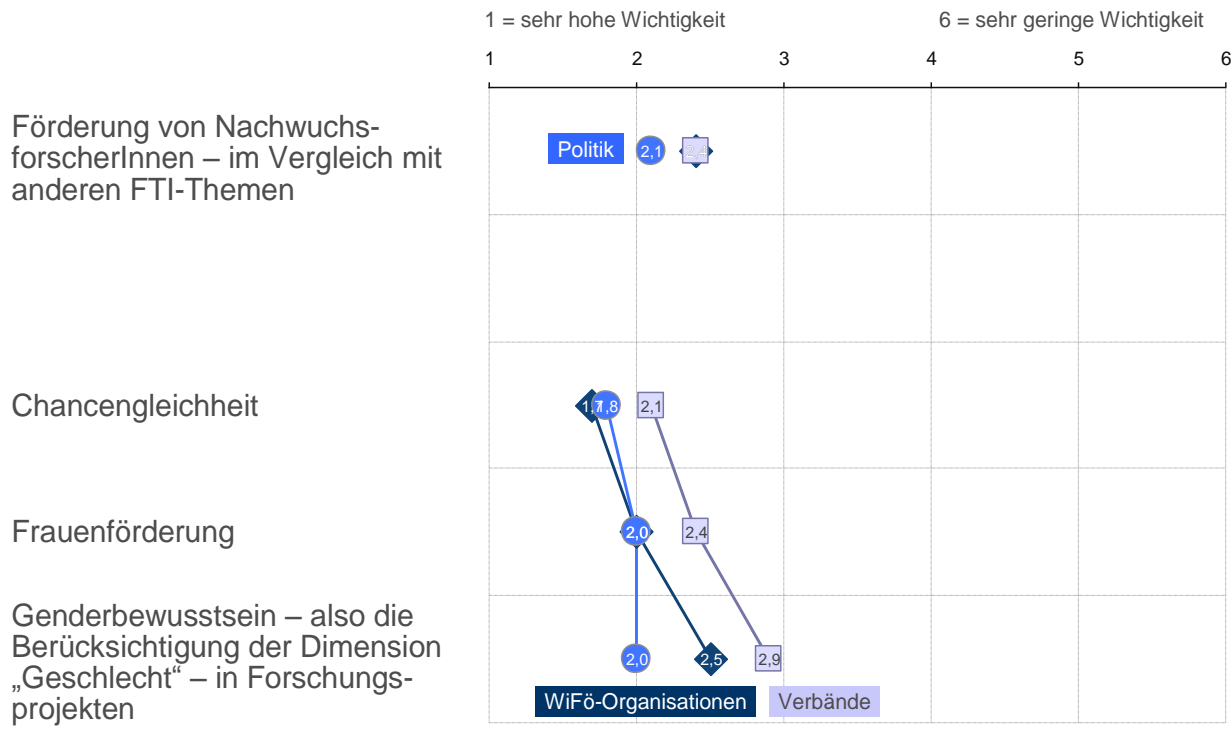

Basis: Alle Befragten $\mathrm{n}=1.972$

Darstellung: Mittelwerte

Frage: Wie schätzen Sie die Wichtigkeit der Förderung von NachwuchsforscherInnen in Österreich im Vergleich mit anderen Forschungs- und Technologiethemen ein? Und wie schätzen Sie die Wichtigkeit des folgenden Themas für FTI in Osterreich ein?

Quellenangabe: ARGE „3C“

Auch die qualitativen Interviews zeigen, dass auch auf der Ebene der FTIpolitischen AkteurInnen Awareness zum Thema Frauenförderung und Chancengleichheit erreicht wurde. Dabei stellen die interviewten AkteurInnen mehrheitlich fest, dass nun „anders über das Thema diskutiert wird“. FTIpolitisch ist damit ein Schub passiert, der Mitte 2010 zur Bündelung in ein „Humanressourcenpaket“ des BMVIT führte. Noch vor wenigen Jahren war Frauenförderung und Chancengleichheit ein Spezialthema weniger Interessierter, heute ist der Handlungsbedarf auf allen Ebenen der Forschungsförderung klar. 
Abbildung 30: Einschätzung der Wirksamkeit von Strategien zur Herstellung von Chancengleichheit durch FTI-politische AkteurInnen

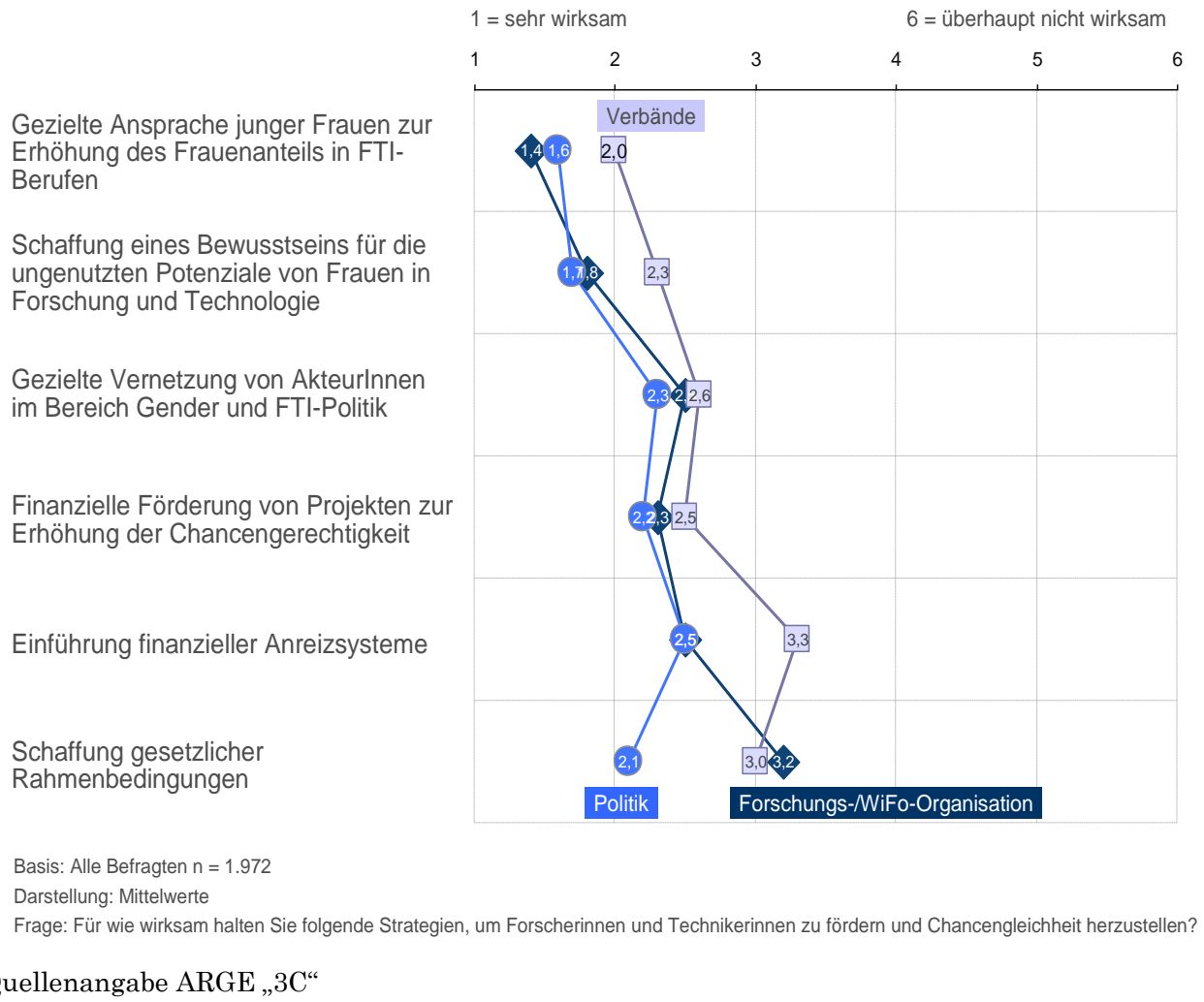

Die Online-Befragung zeigt ferner, dass Verbände generell skeptisch sind bezüglich der Wirksamkeit von Maßnahmen für mehr Chancengleichheit in Forschung, Technologie und Innovation, insbesondere der Einführung von finanziellen Anreizen erteilen sie eine Absage. Forschungs- und Wirtschaftsförderungsorganisationen erachten insbesondere die gezielte Ansprache junger Frauen als wirksam zur Erhöhung des Frauenanteils. AkteurInnen aus der FTI-Politik sehen dies ähnlich, sind zudem im Bereich der Schaffung gesetzlicher Rahmenbedingungen optimistisch. Generell sind die politischen AkteurInnen - und hier denken sie ähnlich wie FTI-Unternehmen und Forschungseinrichtungen (siehe unten) - insbesondere „weichen Maßnahmen“ (Bewusstsein schaffen, sensibilisieren) gegenüber aufgeschlossen. 
Abbildung 31: Einschätzung der Wirksamkeit von Strategien zur Herstellung von Chancengleichheit in FTI - nach FTI-Organisationstyp

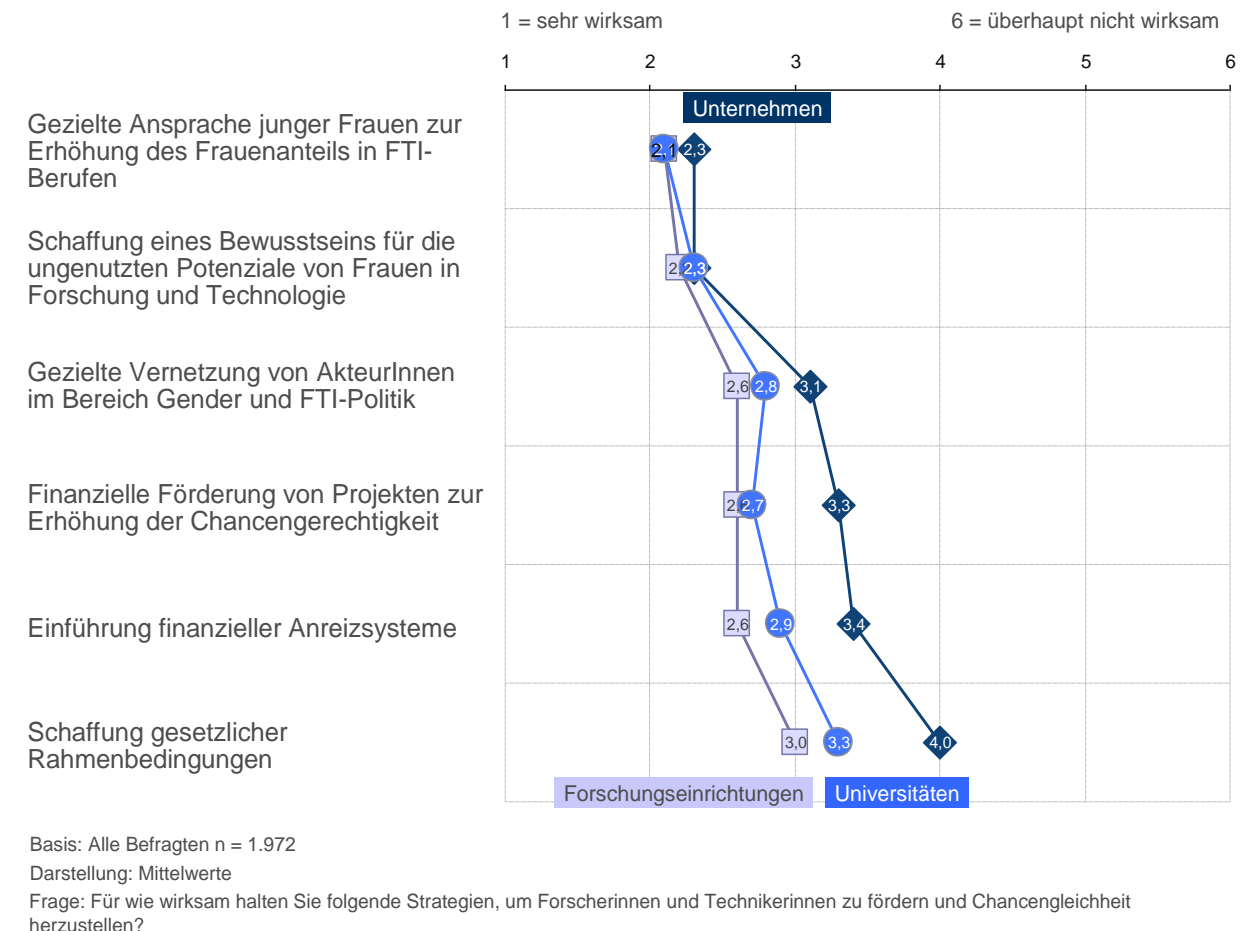

Quellenangabe ARGE „3C“

Betrachtet man in der Onlinebefragung die Akzeptanz der FEMtech Zielsetzungen bei den forschungs- und technologieintensiven Organisationen selbst in diesem Falle Universitäten, Forschungseinrichtungen und Unternehmen dann potenziert sich der oben beschriebene Effekt zwischen dem Ansatz der Chancengleichheit und der Frauenförderung. Während das Zustimmungsniveau zum FEMtech Ziel der Chancengleichheit noch auf demselben Niveau liegt wie bei Politik, FTI-Förderung und Verbänden, so zeigt sich ein zum Teil deutlicher Abfall in der Akzeptanz gezielter Förderung von Frauen. Insbesondere Unternehmen werten hier deutlich schlechter - ein Hinweis darauf, dass sie diesen Ansatz als weniger vereinbar mit ihren Zielen im Personalrecruiting und -management sehen. 
Abbildung 32: Einschätzung der Wichtigkeit von Humanressourcen-Themen nach FTI-Organisationstyp Förderung von Nachwuchs-
forscherlnnen - im Vergleich mit
anderen FTI-Themen

Chancengleichheit

Frauenförderung

Genderbewusstsein - also die Berücksichtigung der Dimension "Geschlecht" - in Forschungsprojekten

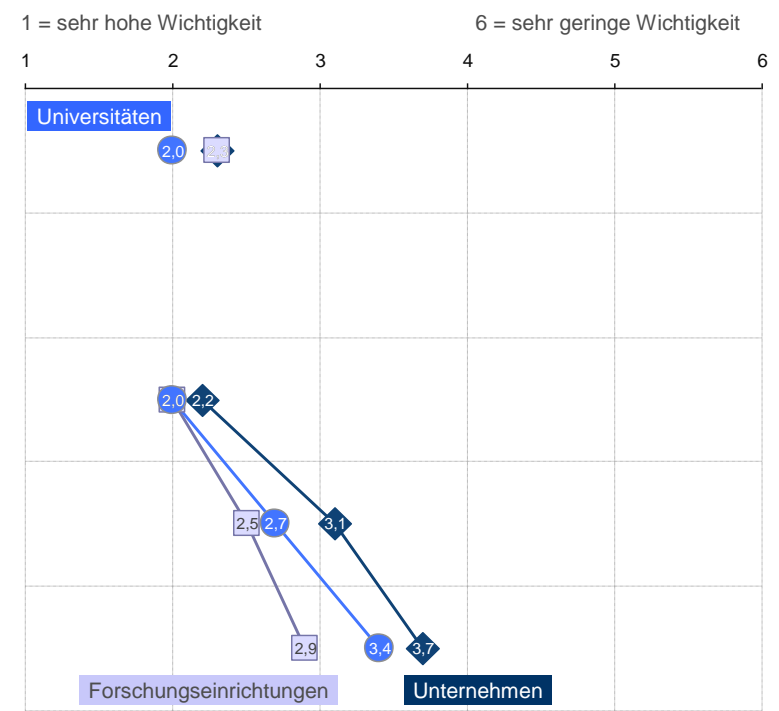

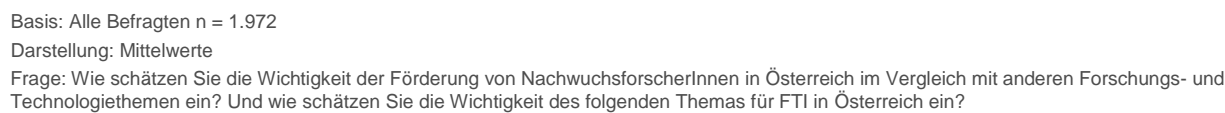

Quellenangabe: ARGE „3C“

FEMtech kann als Programm nicht die FTI-politsche Meinung zu Frauenförderung und Chancengleichheit verändern, sondern als ein wichtiger Baustein gemeinsam mit ähnlichen Maßnahmen versuchen, diese positiv zu beeinflussen. Dass dies z. T. gelungen ist, zeigen die Ergebnisse der Onlinebefragung: Chancengleichheit ist als Thema bei allen AkteurInnen gleichermaßen angekommen und genießt eine ungleich höhere Akzeptanz als Frauenförderung. Dass aber gleichzeitig noch ein langer Weg zu gehen ist, zeigen die Interviews mit den FTI-politischen AkteurInnen. Diese stellen mehrheitlich fest, dass in der FTI-Politik mehr über Frauenförderung und Chancengleichheit gesprochen wird - der Kern der Diskurse jedoch nicht erreicht wird.

\subsection{Conclusio}

\section{Förderungen}

Die FEMtech Förderlinien werden aus Sicht der FTI-politischen AkteurInnen z. T. als verwirrend und zu „theoretisch“ empfunden. Es ist zu wenig konkret und klar, was hier gefördert wird und wozu es hier dreier Programmlinien bedarf. Mit seinen drei Förderlinien spricht FEMtech unterschiedliche inhaltliche Schwerpunkte, z. T. auch verschiedene Zielgruppen an - jedoch nicht differenziert genug. Z. T. finden sich dieselben Unternehmen in den Förderlinien Karriere und Karrierewege, z. T. dieselben Forschungseinrichtungen bei Karriere und Karrierewege, dieselben Universitäten bei Karrierewege und FTI-Projekte. So entsteht zwischen den einzelnen Förderungslinien ein kleiner selbstverweisender Zirkel. 
Im Rahmen der Interviews mit dem oberen Management von FTIUnternehmen und außeruniversitären Forschungseinrichtungen konnten vier Einstellungen bezüglich FEMtech Förderungen eruiert werden.

1. Ablehnung: zum einen inhaltlich begründet, denn es werde keine Qualifizierung von qualifizierten Frauen benötigt bzw. sei eine Differenzierung zwischen Männern und Frauen nicht Unternehmenspolitik; zum anderen in Bezug auf die Antragstellung: Hier passt die Kosten-Nutzen-Relation nicht, da es zu kompliziert und aufwendig ist. (5 von 12 InterviewpartnerInnen)

2. Neutral: „Wir machen hier nichts, weil wir nicht wissen, was FEMtech tut, um unsere Situation zu verbessern“. (Zitat oMan2)

3. Impuls für SpezialistInnen des Förderwesens: Es werden Förderungen der FFG systematisch „gescreent“: „Mit FEMtech ist die Idee entstanden, ein Projekt einzureichen bzw. bietet FEMtech die Ergänzung zu einem bereits bestehenden Projekt". (Zitat oMan4)

4. Chance zur Umsetzung und Systematisierung: Engagierte Personen in (chancengleichheitssensibilisierten) Unternehmen und Forschungseinrichtungen ergreifen die Chance, i) Ideen bzw. geplante Maßnahmen in Umsetzung zu bringen und ii) vorhandene Maßnahmen zu ergänzen und insgesamt das Thema Chancengleichheit im Unternehmen zu systematisieren und gebündelt sichtbar zu machen. (4 von 12 InterviewpartnerInnen)

FEMtech Förderungen sind in ihrer siebenjährigen Geschichte dadurch gekennzeichnet, dass es keine längerfristige Ausschreibungs- und Call-Planung gibt, sondern diese jährlich genehmigt wird. Einzig FEMtech Karriere ist die Förderlinie, die von Anfang an begleitend mitläuft und nicht dem Call-Prinzip unterliegt. Für Unternehmen, die erst für die Themen und Inhalte sensibilisiert werden müssen, ergibt sich eine hohe Planungsunsicherheit, die der Anzahl der Einreichungen bzw. deren Qualität nicht dienlich ist. ${ }^{18}$ Das Programmmanagement in der FFG versucht hier vorbereitend und vorausschauend zu wirken und ermöglicht so, rasch zu reagieren. Dieses Vorgehen bindet jedoch Ressourcen, insbesondere, wenn dann ein Call nicht stattfindet oder langfristig verschoben wird. Die Rollenverteilung zwischen Förderberatung und Einreichberatung - also zwischen FFG und Kompetenzzentrum - ist für die Zielgruppe nicht klar, zumal es bei FEMtech Karriere auch die Akquisitionsbetreuung durch D\&Z Consulting gibt. Dennoch: Die Betreuung und Begleitung der Antragstellenden und Fördernehmenden der FEMtech Programmlinien wird von diesen generell sehr gut bewertet.

FEMtech erreicht mit seinen Förderungen nicht die regionale Breite in Österreich, sondern konzentriert sich auf die „FTI-politischen Kernländer“ Wien, Oberösterreich und Steiermark, im geringeren Ausmaß noch Kärnten. Generell werden auch mit den Förderungen die Chancengleichheits-Interessierten und Sensibilisierten abgeholt, wobei diese eher in den Forschungseinrichtungen und Universitäten zu finden sind. In Zukunft muss daher vermehrt Augenmerk auf die FTI-Unternehmen gelegt werden.

1810 der 12 Self-Assessment-Fragebögen der ProgrammumsetzerInnen stimmen in dieser Einschätzung überein. 


\section{Aktivitäten}

Vorrangig die FEMtech Website, aber auch der Online-Newsletter funktionieren als niedrigschwellige Einstiege ins Programm und sind zugleich wichtiges „Backbone“ zur Bindung der engeren Community. Als umfassende Informations-, Recherche- und Nachrichtenangebote werden sie von FEMtech AdressatInnen stark genutzt und erzielen auch in der FTI-Breite eine solide Nutzung bei guter Bekanntheit.

Die seit Programmstart veranstalteten Netzwerktreffen sind in der gesamten FTI-Community gut bekannt und erreichen ein breites TeilnehmerInnenfeld von rund 1.200 Personen mit hoher Themenaffinität und starker Konzentration auf Wien, welches über diverse Kanäle regelmäßig von FEMtech adressiert wird und aus den Treffen in einem informellen und persönlichen Rahmen positiven Nutzen (mit Abstrichen bei den Unternehmen) gezogen hat.

Beim Forum NaWi)(Tech hingegen liegt bereits die Bekanntheit kaum über der Wahrnehmungsschwelle, was sich auch in der geringen Teilnahme seitens der Studentinnen und Absolventinnen widerspiegelt. Dementsprechend gering ist die Zufriedenheit aufseiten der teilnehmenden Unternehmen, die eine stärkere Resonanz auf ihre Präsentation als potenzieller Arbeitgeber erwarten.

Auszeichnungen erweisen sich als geeignetes Instrument, um mediale Aufmerksamkeit auf das Programm zu lenken und über die Verleihungsveranstaltungen zusätzlich FTI-politische AkteurInnen und MultiplikatorInnen zu erreichen. Der offensichtlich hohe Aufwand, um Unternehmen und Organisationen zu Einreichungen zu bewegen, spricht jedoch dafür, dass die Anzahl der unterschiedlichen Preise im Rahmen von FEMtech zu groß ist (FEMtech meets Fiction, Staatspreis für Chancengleichheit in F\&E und Gender Award), insbesondere, da Maßnahmen zur Frauenförderung und Chancengleichheit auch im Rahmen anderer Preisverleihungen prämiert werden (bspw. die Auszeichnung für die frauen- und familienfreundlichsten Betriebe durch http://www.taten-statt-worte.at/. Weitere Preise in der Kategorie "Gender“ können über http://www.awards.at/ recherchiert werden).

Auch mit der „Expertin des Monats“ erzielt FEMtech erfolgreich Medienpräsenz und sorgt für eine stärkere öffentliche Präsentation erfolgreicher Frauen in der Breite. Die zugrunde liegende „Expertinnen-Datenbank“ profitiert davon jedoch wenig - ihre NutzerInnenschaft beschränkt sich stark auf die Expertinnen selbst bzw. eine enge Community aus Hochschule und Forschung. Aufgrund der geringen Zahl tatsächlicher Anfragen dient die Datenbank registrierten Expertinnen bis dato kaum als Plattform zur Vermittlung ihrer Fachkompetenzen.

Das Portfolio von FEMtech im Bereich Aktivitäten entspricht dem internationalen Standard an Awareness- und Service-Angeboten. Im Vergleich mit den Fallbeispielen fällt die prominente Präsentation der „Expertin des Monats“ auf der Website positiv auf. Zur Sichtbarmachung von Frauen in Forschung und Entwicklung setzen das UKRC und das ABI noch stärker auf die Herausstellung der Exzellenz einzelner Wissenschafterinnen. Beide Einrichtungen vergeben Preise für herausragende Frauen in verschiedenen Kategorien aus dem Wissenschafts-, Ingenieurs- und Technologiesektor. Auf dem Gebiet der Vernetzung sind die Angebote der internationalen Einrichtungen vielfältiger und 
zielgruppenspezifischer. So bietet das UKRC beispielsweise Netzwerke für Frauen, für Frauenorganisationen sowie für Fachgesellschaften an. An Vernetzung oder Unterstützung interessierte Personen werden beim ABI und beim UKRC direkt über die Webseite über „get involved“ angesprochen. Zudem nutzen beide Einrichtungen auch soziale Netzwerke, wie z. B. Facebook und Twitter.

\section{Wissen}

FEMtech hat sein Ziel, Sammelpunkt für Daten und Fakten, Studien und Publikationen aus dem Bereich Frauen in Forschung und Technologie zu sein, erreicht. Wie sich durch die sehr guten Ergebnisse der Online-Befragung im Bereich Awareness und auch aus dem Feedback der Interviews ergeben hat, stellt FEMtech einen sehr umfassenden Informationspool im Bereich Daten und Publikationen für das Thema „Frauen in F\&E“ zur Verfügung.

Die Auswertung der Online-Befragung und der Interviews lässt allerdings darauf schließen, dass insbesondere hinsichtlich des Datenmaterials einerseits ein Sättigungsgrad erreicht ist, andererseits die relevanten Informationen nur einem kleinen Kreis von Nutzerinnen und Nutzern zugänglich sind. Das zweite Ziel im Bereich Wissen, Informationen zum Thema „Frauen in F\&E“ aufzubereiten und leicht zugänglich zu machen, hat FEMtech nur zum Teil erfüllt. Positiv ist hervorzuheben, dass sowohl die eigenen als auch die externen $\mathrm{Pu}$ blikationen in der Regel zum Download zur Verfügung stehen. Der Aufbau der Navigation sowie die Benennung der einzelnen Seiten erschweren jedoch den Zugang zu dem umfangreichen und breit gefächerten Angebot. Zudem sind die Informationsangebote nicht hinreichend strukturiert, z. B. nach Themen aufbereitet. Der seit Dezember 2010 mit dem neuen Unterpunkt „Themen“ verfolgte Ansatz greift die oben genannten Probleme auf und strukturiert das Informationsangebot für drei ausgewählte Themenbereiche übersichtlich. Es ist jedoch nicht klar, wie sich diese Aufbereitung der Informationsangebote in die bisherige Struktur einfügt.

Wie bereits in der Zielgruppenanalyse in Kapitel 2 „FEMtech Programmlogik und -konzeption" deutlich wird, fehlt es auch für den Bereich Wissen an einem zielgruppenspezifischen Angebot. So sind außeruniversitäre Forschungseinrichtungen beispielsweise eher an internationalen Good-Practice-Beispielen interessiert, während Unternehmen ein Bedürfnis nach praxisorientierten Aufarbeitungen und Empfehlungen haben. Hinsichtlich des Themenspektrums ist auffällig, dass das Angebot an Download-Literatur auf der FEMtech Website sehr umfassend ist. Die FEMtech eigenen Publikationen bedienen jedoch nicht alle Themen entsprechend der eigenen Zielhierarchie und die Dokumentation der eigenen Maßnahmen nimmt einen großen Raum ein. Dieser Fokus auf die eigenen Maßnahmen und Produkte schwächt das Profil von FEMtech als Kompetenzzentrum für den Bereich Frauen in F\&E. Die forschungs- und theoriebasierte Weiterentwicklung des Programms erfordert eine differenzierte Auseinandersetzung mit den nur langsam steigenden Zahlen von Frauen in F\&E, insbesondere in Führungspositionen, und den veränderten gleichstellungspolitischen Erkenntnissen.

Im Verhältnis zu den internationalen Fallbeispielen ist das Daten- und Publikationsangebot von FEMtech sehr umfangreich. Insbesondere die vielen Down- 
load-Angebote sind positiv zu bewerten. Allerdings ist z. B. das UKRC diesbezüglich noch serviceorientierter, indem es Informationen zu Netzwerken, Organisationen, Videos, Publikationen usw. in einer Online-Datenbank zur Verfügung stellt. 


\section{FEMtech Effekte und Wirkungen}

Erkenntnisse zu Wirkungen von FEMtech im Sinne der Zielerreichung stammen aus folgenden Quellen:

1. flächendeckende Online-Befragung (Vollerhebung) von FEMtech Adressatinnen $(\mathrm{n}=352)$ und Fördernehmenden $(\mathrm{n}=37)$ sowie - als Vergleichsgruppe dazu - generell von Projektleitungen aus FTI-Unternehmen und -Organisationen, die seit 2004 FFG-Forschungsförderung beantragt haben $(\mathrm{n}=1.583)$

2. qualitative Interviews mit VertreterInnen des oberen Managements aus FTI-Unternehmen/-Forschungseinrichtungen, FTI-politischen AkteurInnen, FEMtech geförderten Frauen sowie aktuellen und ehemaligen FEMtech ProgrammleiterInnen.

Während es bei Ersteren um die Einschätzung von organisationsinternen und österreichweiten Veränderungen durch die Nutzung von FEMtech Angeboten und -Förderungen geht, liefern die Interviews qualitative Erklärungsansätze.

\subsection{FEMtech Bekanntheit}

Die Voraussetzung für jegliche Form von Wirkung ist die grundsätzliche Erreichung der Zielgruppen bzw. deren Kenntnis des Programms.

Unter den FEMtech AdressatInnen ist die Reichweite - wenig erstaunlich mit $96 \%$ nahezu vollständig, wie die Online-Befragung zeigt. Aber immerhin auch $44 \%$ der Befragten aus FTI-Unternehmen und -Organisationen (FFG) kennen FEMtech als Programm zumindest dem Namen nach.

Zieht man die Fülle der vorhandenen Programme im FTI-Kontext in Betracht, erscheint die Bekanntheit von FEMtech damit durchaus bemerkenswert.

Hinzu kommt, dass das Thema "Chancengleichheit“ von allen AkteurInnen (Unternehmen, Forschungseinrichtungen, Hochschulen, FTI-Policy) als wichtig erachtet wird - dies zeigt die Onlinebefragung (Einschätzung der Wichtigkeit je nach Gruppe zwischen 2,0 und 2,2 auf einer Sechser-Skala) ebenso wie die qualitativen Interviews mit allen befragten Zielgruppen. Insbesondere die FTIpolitischen AkteurInnen konstatieren in den Interviews, dass Chancengleichheit „als Thema präsenter geworden ist, es wird jetzt anders darüber diskutiert“ (Zitat FTI-pA1). FEMtech habe hier (nicht allein, sondern neben parallel laufenden Programmen etwa im Rahmen fFORTE) einen wichtigen Beitrag zum Agenda-Setting geleistet. Gleichzeitig merken die Befragten aber auch an, dass Chancengleichheit als Thema „noch nicht im Kern des FTI-politischen Diskurses angelangt" (Zitat FTI-pA4) ist und bisher erst kleine Schritte gegangen wurden. „Die Community, die sich um das Thema kümmert, bleibt weiter unter sich."(Zitat FTI-pA2)

Von den VertreterInnen des oberen Managements kommt darüber hinaus auch der Hinweis, dass FEMtech als Frauenförderungs- und nicht als Chancengleichheitsprogramm wahrgenommen wird. 


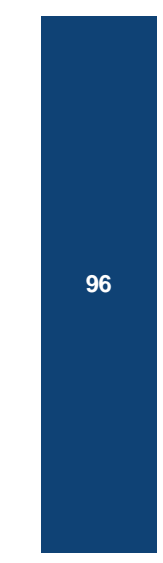

FEMtech Adressatlnnen

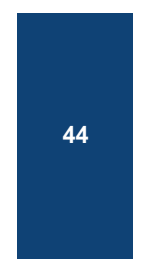

FTI-Unternehmen und Organisationen (FFG)

Basis: Alle Befragten; $\mathrm{n}=352$ (FEMtech AdressatInnen) / 1.583 (FTI FFG)

Darstellung: Prozentwerte

Frage: Kennen Sie „FEMtech“, ein Programm des bmvit zur Förderung der Chancengleichheit in Forschung und Technologie - sei es auch nur dem Namen nach?

Quellenangabe: ARGE „3C“

\subsection{FEMtech Zielgruppenerreichung}

Bei der Frage, welche Zielgruppen FEMtech über alle Maßnahmenbereiche und Einzelinstrumente hinweg als Gesamtprogramm tatsächlich erreicht, ist mehr als die reine Namensbekanntheit des Programms zugrunde zu legen. Grundlage für die folgenden Analysen ist die Online-Befragung. Als „erreicht“ gelten im Folgenden Personen, die FEMtech nicht nur kennen, sondern auch tatsächlich Angebote nutzen. Dabei sind bei der Analyse der Nutzung zwei Betrachtungsebenen wichtig:

1. das Profil der FEMtech NutzerInnen - also Zusammensetzung der Gruppe der FEMtech NutzerInnen in Bezug auf bestimmte soziodemografische Merkmale -> beantwortet die Frage: „Wer sind die FEMtech NutzerInnen bzw. wie kann man die Zusammensetzung der Gruppe beschreiben?“

2. der Anteil der FEMtech NutzerInnen innerhalb bestimmter soziodemografischer Gruppen (= Anteil der FEMtech NutzerInnen an der Gesamtheit dieser spezifischen Gruppe) -> beantwortet die Frage: „In welchem Umfang werden einzelne soziodemografische Subgruppen durch FEMtech erreicht?"

Als FEMtech NutzerInnen gelten hier Personen, die mindestens ein FEMtech Angebot nutzen - unabhängig davon, ob es sich um Förderungen, Aktivitäten oder Wissensangebote handelt. Ebenso ist es hier unerheblich, aus welcher Befragtengruppe die NutzerInnen stammen, d. h. ob sie als FEMtech Adressatin durch das Programm regelmäßig zur Nutzung eingeladen werden oder aber quasi „von selbst“ als VertreterIn der FTI-Unternehmen und Organisationen (FFG) darauf gestoßen sind. 
Um die unterschiedliche Intensität und Qualität der Nutzung ebenfalls abzubilden, wird zusätzlich zwischen Intensiv-NutzerInnen (= mindestens vier Angebote genutzt, darunter mindestens eine Förderung oder eine Veranstaltung) und Sporadisch-NutzerInnen (= FEMtech NutzerInnen, die nicht Intensiv-NutzerInnen sind) unterschieden.

\section{Profil der FEMtech NutzerInnen}

Drei Viertel aller FEMtech NutzerInnen sind FTI-Unternehmen und -Organisationen. Innerhalb dieser Gruppe haben privatwirtschaftliche Unternehmen den höchsten Anteil (37\%), gefolgt von Universitäten (29 \%) und Forschungseinrichtungen (18\%). Allerdings werden NutzerInnen der letzten beiden Gruppen deutlich intensiver erreicht als die aus Unternehmen.

Die meisten NutzerInnen (zusammengenommen $65 \%$ mit einem Schwerpunkt in der Größenklasse 10-49 MitarbeiterInnen) stammen aus KMU mit unter 250 MitarbeiterInnen. Mit $21 \%$ sind jedoch auch die Großunternehmen mit mehr als 1.000 Mitarbeitenden stark vertreten. Separat betrachtet, machen sie nach den genannten Kleinunternehmen (10 bis 49 MA) die zweitgrößte Gruppe aus.

Tabelle 30: Profil FEMtech NutzerInnen

\begin{tabular}{l|c|c|c} 
& $\begin{array}{c}\text { FEMtech } \\
\text { NutzerInnen } \\
\text { gesamt }\end{array}$ & $\begin{array}{c}\text { Intensiv- } \\
\text { NutzerInnen }\end{array}$ & $\begin{array}{c}\text { Sporadisch- } \\
\text { NutzerInnen }\end{array}$ \\
\hline Basis: alle Befragten & $\mathrm{n}=612$ & & \\
\hline & davon $\rightarrow$ & $\mathrm{n}=235$ & $\mathrm{n}=377$ \\
\hline Art der Organisation & & & \\
\hline FTI-Organisation & $76 \%$ & $67 \%$ & $82 \%$ \\
\hline $\begin{array}{l}\text { Forschungs- oder Wirtschafts- } \\
\text { förderungsorganisationen }\end{array}$ & $3 \%$ & $5 \%$ & $2 \%$ \\
\hline Verband / Interessenvertretung & $2 \%$ & $2 \%$ & $1 \%$ \\
\hline Politik / öffentliche Verwaltung & $5 \%$ & $8 \%$ & $3 \%$ \\
\hline Sonstige Organisation & $8 \%$ & $10 \%$ & $7 \%$ \\
\hline StudentIn & $2 \%$ & $2 \%$ & $2 \%$ \\
\hline MedienvertreterIn & $1 \%$ & $3 \%$ & $0 \%$ \\
\hline Sonstiges & $3 \%$ & $4 \%$ & $2 \%$ \\
\hline Größe der Organisation & & & \\
\hline bis 9 MitarbeiterInnen & $17 \%$ & $11 \%$ & $19 \%$ \\
\hline 10 bis 49 & $29 \%$ & $25 \%$ & $32 \%$ \\
\hline 50 bis 249 & $19 \%$ & $19 \%$ & $19 \%$ \\
\hline 250 bis 499 & $7 \%$ & $9 \%$ & $6 \%$ \\
\hline 500 bis 999 & $7 \%$ & $10 \%$ & $5 \%$ \\
\hline mehr als 1.000 MitarbeiterInnen & $21 \%$ & $26 \%$ & $18 \%$ \\
\hline Dastl & & & \\
\hline
\end{tabular}

Darstellung in \%, Prozentuierung auf Basis Antwortende (d. h. ohne „keine Angabe/weiß nicht“) 


\begin{tabular}{|c|c|c|c|}
\hline & $\begin{array}{l}\text { FEMtech- } \\
\text { Nutzende } \\
\text { Gesamt }\end{array}$ & $\begin{array}{l}\text { Intensiv } \\
\text { Nutzende }\end{array}$ & $\begin{array}{l}\text { Sporadisch } \\
\text { Nutzende }\end{array}$ \\
\hline \multicolumn{4}{|l|}{$\begin{array}{l}\text { Basis: Nur Befragte aus FTI- } \\
\text { Organisationen }\end{array}$} \\
\hline & $\mathrm{n}=465$ & & \\
\hline & davon $\rightarrow$ & $\mathrm{n}=156$ & $\mathrm{n}=309$ \\
\hline \multicolumn{4}{|l|}{ Art der FTI-Organisation } \\
\hline $\begin{array}{l}\text { Privatwirtschaftliches Unterneh- } \\
\text { men }\end{array}$ & $37 \%$ & $26 \%$ & $42 \%$ \\
\hline $\begin{array}{l}\text { Außeruniversitäre und kooperative } \\
\text { Forschungseinrichtung }\end{array}$ & $18 \%$ & $25 \%$ & $15 \%$ \\
\hline Universität & $29 \%$ & $29 \%$ & $29 \%$ \\
\hline Fachhochschule & $5 \%$ & $6 \%$ & $4 \%$ \\
\hline Kompetenzzentrum oder -netzwerk & $6 \%$ & $8 \%$ & $5 \%$ \\
\hline Intermediäre Einrichtung & $0 \%$ & $0 \%$ & $0 \%$ \\
\hline Verein & $4 \%$ & $4 \%$ & $4 \%$ \\
\hline Sonstiges & $1 \%$ & $1 \%$ & $1 \%$ \\
\hline
\end{tabular}

Darstellung in \%, Prozentuierung auf Basis Antwortende (d.h. ohne „keine Angabe/weiß nicht“)

Quellenangabe: ARGE „3C“

Regional fällt die extrem starke Fokussierung auf Wien auf: Die Hälfte aller FEMtech NutzerInnen arbeitet in der Hauptstadt.

Dass FEMtech als Programm zur Förderung von Frauen in Forschung und Technologie eher Frauen als Männer anspricht, ist naheliegend. Tatsächlich sind rund 2/3 der NutzerInnen weiblich (bei Intensiv-NutzerInnen sogar $80 \%$ ).

Im Hinblick auf Branchen sind die NutzerInnen breit gestreut. Ein Vergleich mit dem Befragungsrücklauf zeigt, dass sich die Branchenzugehörigkeit der FEMtech NutzerInnen darin im Großen und Ganzen widerspiegelt. In Bezug auf Branchen stellen die erreichten NutzerInnen also einen guten Querschnitt dar.

Bezogen auf die Merkmale „Alter“ und „Position“ zeigt sich bei den FEMtech NutzerInnen ebenfalls ein sehr ausgewogenes Bild, welches weitgehend der Verteilung in der Gesamtheit aller Befragten entspricht. FEMtech erreicht darüber hinaus deutlich eher Angestellte (82 \%) als Selbstständige (15\%).

Der/die typische FEMtech NutzerIn lässt sich also wie folgt charakterisieren: Weiblich, angestellt, aus Wien, eher tätig im Unternehmen mit unter 250 Mitarbeitenden.

\section{Welche Gruppen erreicht FEMtech in welchem Umfang?}

Die Charakterisierung der/des typischen FEMtech NutzerInnen im vorangegangenen Abschnitt sagt per se noch nichts darüber aus, ob bestimmte Gruppen bezogen auf ihre Gesamtheit gut oder schlecht erreicht wurden. Dies verdeutlicht das Beispiel der Unternehmen: Zwar machen VertreterInnen aus Unternehmen das Gros der FEMtech NutzerInnen aus (siehe vorangegangenen 
Abschnitt), dennoch wird diese Gruppe, bezogen auf die Gruppe der insgesamt von der FFG erreichten Unternehmen und im Vergleich zu Forschungseinrichtungen und Universitäten, deutlich unterdurchschnittlich erreicht (siehe unten).

Betrachtet man zunächst die drei Online-Befragtengruppen, zeigt sich, dass FEMtech Adressatinnen und -Fördernehmende - entsprechend ihrer Nähe zum Programm - umfassend und größtenteils intensiv erreicht werden. Bei den Fördernehmenden wird deutlich, dass diese nicht ausschließlich die Förderung entgegennehmen, sondern zum größten Teil auch weitere Angebote des Programms nutzen.

Im Gegensatz dazu werden FTI-Unternehmen und -Organisationen (FFG) in der Breite nur zu $17 \%$ erreicht - wobei die Nutzung dann zum größten Teil auch nur sporadisch ausfällt.

Abbildung 34: FEMtech NutzerInnen - nach Befragtengruppe

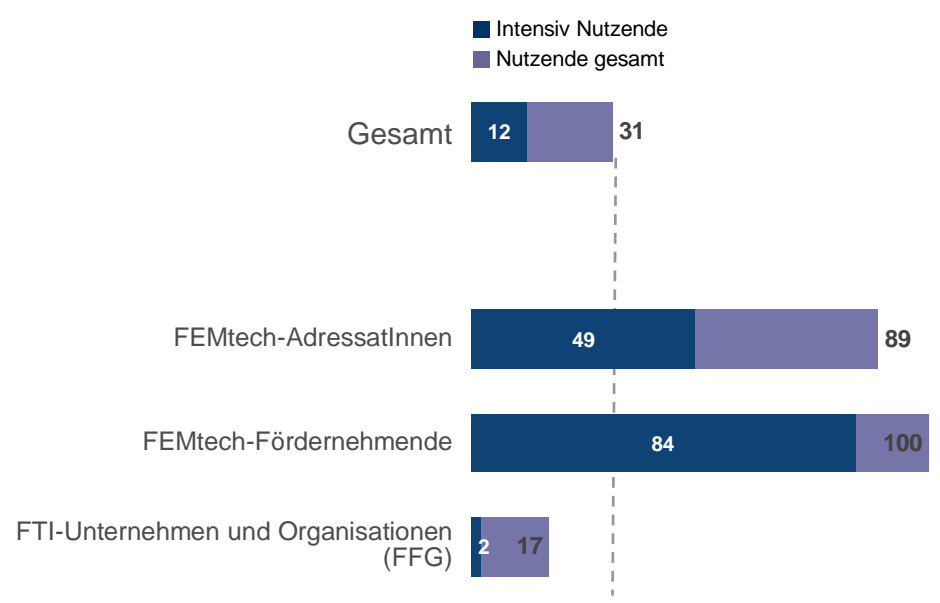

Basis: Alle Befragten, $\mathrm{n}=1.972$ FEMtech Nutzende $=$ mind. 1 Angebot aus Bereichen
Förderung, Aktivität oder Wissen genutzt Darstellung: Prozentwerte

Quellenangabe: ARGE „3C“

Bezogen auf die Merkmale Geschlecht, Alter und Arbeitsort bestätigt sich das bereits beim Profil der NutzerInnen ermittelte Bild - FEMtech erreicht vor allem die Frauen und ist regional sehr stark auf Wien fokussiert. So nutzen $63 \%$ aller weiblichen Befragungsteilnehmenden FEMtech. Und während WienerInnen zu $46 \%$ erreicht werden, fällt die Nutzung in allen anderen Bundesländern z. T. gering aus. Lediglich in der Steiermark, in Tirol und in Kärnten liegt die Nutzungsrate noch nah am Gesamtdurchschnitt.

Altersmäßig ist der NutzerInnenanteil relativ ausgewogen mit leichter Tendenz der besseren Erreichung jüngerer Personen (37\% NutzerInnen unter 30 Jahren).

In der Betrachtung der erreichten Positionen fällt ein leichter Einschnitt bei der mittleren Hierarchiestufe auf (nur 23 \% NutzerInnen auf der Ebene Abteilungsleitung). Am besten erreicht werden Personen ohne oder mit sonstiger Führungsverantwortung, aber auch die Organisationsleitungen werden in einem guten Ausmaß erreicht (34\% NutzerInnen). 
Hinsichtlich des Organisationstyps zeigt sich einmal mehr die sehr gute Präsenz von FEMtech in Hochschulen und Forschungseinrichtungen und die geringe in privatwirtschaftlichen Unternehmen. Weiters sieht man auch, dass Kompetenznetzwerke/Cluster sowie Vereine einen überdurchschnittlichen Anteil an FEMtech NutzerInnen aufweisen.

Während die/der typische FEMtech NutzerInnen aus der Gruppe der KMU (weniger als 250 Mitarbeitende) kommt (siehe oben: Profil der NutzerInnen), zeigt sich bei der Betrachtung des Anteils ein gegenteiliges Bild mit klarer und durchgängiger Tendenz: Je mehr MitarbeiterInnen eine Organisation hat, desto höher ist der Anteil von FEMtech NutzerInnen (35 \% in Organisationen mit mehr als 1.000 MitarbeiterInnen). Tendenziell werden also MitarbeiterInnen aus großen Organisationen (seien es privatwirtschaftliche Unternehmen, Forschungseinrichtungen oder Universitäten) besser, insbesondere intensiver erreicht.

Darüber hinaus sind die NutzerInnen in der Gruppe der Selbstständigen unterrepräsentiert (16\%).

Abbildung 35: FEMtech NutzerInnen in FTI-Unternehmen/-Organisationen nach FTI-Organisationstyp

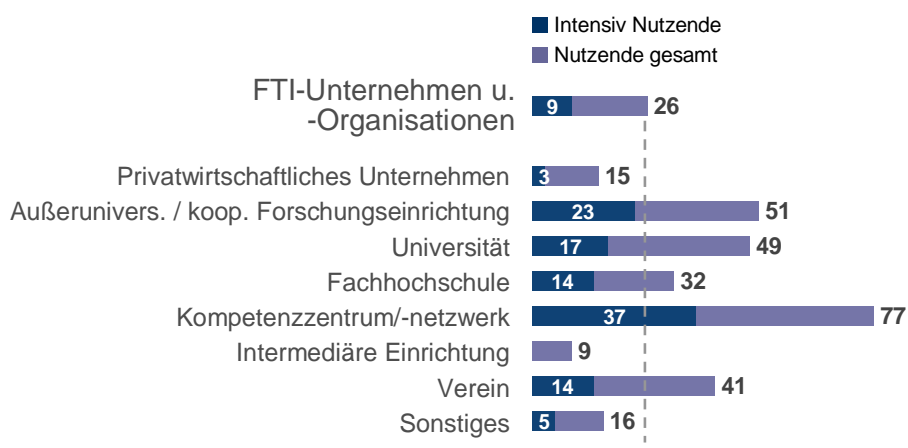

Basis: Alle Befragten aus FTI-Organisationen, $n=1.806$ Darstellung: Prozentwerte

FEMtech Nutzende $=$ mind. 1 Angebot aus Bereichen Förderung, Aktivität oder Wissen genutz Intensiv Nutzende $=$ mind. 4 Angebote genutzt, darunter mind. 1 Förderung oder Veranstaltung

Quellenangabe: ARGE „3C“

In Bezug auf Branchen fällt auf, dass weiterverarbeitende und produzierende Industriebereiche (z. B. Bauwirtschaft, Maschinenbau und Elektrotechnik) unterdurchschnittlich erreicht werden. Gut und intensiv erreicht werden hingegen Branchen, die bereits über einen höheren Frauenanteil in den entsprechenden Studienrichtungen und z. T. auch bei den Mitarbeitenden verfügen, z. B. Land- und Forstwirtschaft/Veterinärmedizin, Umwelt, Biowissenschaften/Lebensmitteltechnik oder Gesundheitswesen (inkl. Humanmedizin und Medizintechnik).

Zusammengefasst erreicht FEMtech folgende Gruppen gut: Frauen, Angestellte, WienerInnen, in Forschungseinrichtungen, Universitäten oder Kompetenzzentren Tätige, in organisationsleitender Funktion oder als MitarbeiterIn mit/ohne Führungsverantwortung, in Organisationen mit mind. 500 Mitarbeitenden und in Branchen mit stärkerer Frauenpräsenz. 
Unterdurchschnittlich erreicht werden privatwirtschaftliche Unternehmen und KMU.

\subsection{Durch FEMtech erzielte Veränderungen (Outcomes)}

Grundlage für die Analyse der durch FEMtech bei den Zielgruppen bewirkten Veränderungen (Outcomes) ist in erster Linie die Online-Befragung, ergänzt um Erkenntnisse aus qualitativen Interviews mit Zielgruppen. Alle Befragten aus FTI-Unternehmen und -Organisationen wurden $\mathrm{zu}$ von ihnen wahrgenommenen Veränderungen in sämtlichen Dimensionen abgefragt, die zum FEMtech Zielkatalog gehören. In der Analyse wurde dann jeweils für Förderungen, Aktivitäten und Wissen die jeweilige konkrete NutzerInnengruppe einer Vergleichsgruppe von Nicht-NutzerInnen gegenübergestellt. Bei Zieldimensionen, die die Veränderungen innerhalb von Organisationen betreffen (insbesondere betrifft dies die Förderungen) wurde zudem die Vergleichsgruppe strukturell der Gruppe der Geförderten angepasst (siehe unten).

Bei den folgenden Darstellungen ist zu beachten, dass sich die Analyse der Outcomes allein auf wahrgenommene Veränderungen stützen muss. Es fehlen belastbare Zahlen zur Ausgangslage vor den Interventionen durch FEMtech, wie sie beispielsweise eine Nullmessung bei Programmstart hätte liefern können.

\subsubsection{Outcomes FEMtech Förderungen}

Für die Analyse der durch FEMtech Förderungen erzielten Veränderungen (Outcomes) bei den Zielgruppen wurde die Vergleichsgruppe der FTIOrganisationen und -Unternehmen (FFG) an die jeweilige Struktur der Fördernehmenden in den drei Förderlinien angepasst. Dazu wurde die online befragte Vergleichsgruppe (FTI-Unternehmen/Organisationen, die seit 2004 FFG-Förderungen beantragt haben) um Organisationstypen bereinigt, die nicht Zielgruppe der Förderung sind. Zusätzlich wurde per Gewichtung die proportionale Verteilung der verbliebenen FTI-Organisationen in der Vergleichsgruppe hinsichtlich Organisationstyp und -größe an die Struktur der Fördernehmenden angeglichen.

Auf diese Weise ist größtmögliche Vergleichbarkeit zwischen Fördernehmenden und den jeweiligen Vergleichsgruppen gewährleistet. Unterschiede im Antwortverhalten, so die hier zugrunde liegende Annahme, müssen auf andere Faktoren (hier: Intervention durch Förderung) zurückzuführen sein und können nicht an unterschiedlichen Gruppenzusammensetzungen liegen.

\section{Outcomes FEMtech Karriere}

Fördernehmende (befragt wurden die Projektleitungen sowie weitere MitarbeiterInnen geförderter Organisationen ${ }^{19}$ ) von FEMtech Karriere konstatieren

\footnotetext{
${ }^{19} \mathrm{Als}$ separate Gruppe explizit zur Online-Befragung eingeladen wurden nur die Projektleitungen. Jedoch kamen im Zuge der Online-Befragung weitere MitarbeiterInnen geförderter Institutionen hinzu (etwa aus dem FEMtech AdressatInnenkreis). Deren Antworten stellen eine Kontrolle sowie ein sinnvolles Korrektiv gegenüber dem alleinigen Urteil der Projektleitungen dar, denn Letztere
} 
deutliche Veränderungen in der eigenen Organisation innerhalb der letzten fünf Jahre und urteilen bezogen auf alle durch die Förderlinie intendierten Resultate deutlich positiver als die Vergleichsgruppe. Die erzielten Verbesserungen bewegen sich jedoch je nach Zieldimension auf unterschiedlichem Niveau.

Insbesondere bezogen auf „weichere“ Faktoren der Unternehmenskultur konnten in den geförderten Organisationen der Gedanke der Chancengleichheit erfolgreich verankert werden und damit einhergehend auch Führungskräfte für das Thema sensibilisiert werden. Dies bestätigen auch die qualitativen Interviews mit VertreterInnen des oberen Managements: Durch die FEMtech Förderung konnte das Thema Chancengleichheit forciert und im Unternehmen besser dargestellt werden. Klar wird aus den Interviews allerdings auch, dass in den geförderten Unternehmen und Forschungseinrichtungen bereits vorab eine Sensibilisierung für das Thema bestanden hat. Durch die FEMtech Förderung konnten jedoch vorhandene Ideen aufgegriffen und umfassender umgesetzt werden oder aber bestehende Maßnahmen ergänzt oder systematisiert werden. Diese Meinung teilten alle neun der zwölf InterviewpartnerInnen, welche schon Förderungen im Rahmen von FEMtech in Anspruch genommen hatten.

Ein geteiltes Bild zeigt sich bei den Dimensionen, die auf strukturelle Verbesserungen und die Erhöhung des Frauenanteils auf verschiedenen Ebenen abzielen. Während das der Chancengleichheit zuzuordnende Ziel einer Verbesserung der Work-Life-Balance (Mw. 1,8) sehr gut erreicht wurde, fallen die Veränderungen im Bereich der gezielten Frauenförderung - in diesem Falle die Erhöhung des Frauenanteils an FTI-Neueinstellungen (Mw. 2,6) und in Führungspositionen (Mw. 3,4) - eher mittel bis ziemlich gering aus. Auch die Interviewten aus dem oberen Management sehen Verbesserungen auf der strukturellen Ebene, insbesondere im Bereich der Bewerbungsverfahren und der Personalrekrutierung, was sich ebenfalls nicht zwangsläufig in einem erhöhten Frauenanteil niederschlagen muss.

Interessant ist das Ergebnis in Bezug auf das Ziel interner organisatorischer Veränderungen zur stärkeren Förderung von Frauen: Zwar liegen die geschätzten Veränderungen bei den geförderten Organisationen nur auf mittlerem Niveau (Mw. 2,8) - es handelt sich hier jedoch zugleich um den Aspekt, bei dem die höchste Diskrepanz zwischen Geförderten und Vergleichsgruppe deutlich wird. Dies könnte ein Indikator dafür sein, dass die Veränderungen bei den Fördernehmenden durchaus in beträchtlichem Umfang stattfanden, jedoch noch nicht als ausreichend empfunden werden.

Die starken Differenzen in der Einschätzung zwischen geförderten und nichtgeförderten Organisationen lassen bereits darauf schließen, dass diese zumindest in Teilen der FEMtech Förderung zugerechnet werden können. Tatsächlich schätzen die Befragten den Beitrag von FEMtech Karriere zu den wahrgenommenen internen Veränderungen mit $67 \%$ als recht hoch ein.

haben womöglich auch Interesse, Effekte günstig darzustellen und antworten ggf. eher „strategisch“. Insofern fließen in die Ergebnisse zu Outcomes in den geförderten Unternehmen sowohl die Antworten der Projektleitung als auch die weiterer MitarbeiterInnen ein. 
In Ergänzung dazu zeigen die Interviews mit dem oberen Management aus FTI-Organisationen, die Förderungen erhalten haben, zwei generelle Wirkmechanismen bei FEMtech Karriere auf: Wenn Chancengleichheit institutionell nur ein Nebenthema und dementsprechend wenig verankert ist, liegt es an dem Engagement von Einzelpersonen, das Thema zu transportieren und Maßnahmen zu setzen. Wenn aber bereits organisationsintern eine erhöhte Sensibilität für das Thema besteht, sorgt die Förderung für eine Dynamisierung des Implementierungsprozesses - Maßnahmen werden dann schneller und umfassender umgesetzt.

Bei der Interpretation der Unterschiede zwischen Geförderten und Vergleichsgruppe gilt bei „Karriere“, aber auch bei den beiden in den folgenden Kapiteln dargestellten Förderlinien folgende Einschränkung: Es lassen sich keine im statistischen Sinne belastbaren Aussagen dazu machen, in welchem Umfang die Unterschiede auf FEMtech zurückzuführen sind bzw. ob ohne FEMtech Förderung nicht womöglich auch ein Unterschied zwischen den beiden Gruppen bestanden hätte. Da die Förderung vor allem bereits affine Unternehmen und Organisationen erreicht hat (siehe oben) liegt letztere Vermutung durchaus nah. Der hohe Anteil, den die Förderung laut der Befragten an den Veränderungen hatte, relativiert diese Aussage allerdings wieder.

Abbildung 36: Zielerreichung FEMtech Karriere

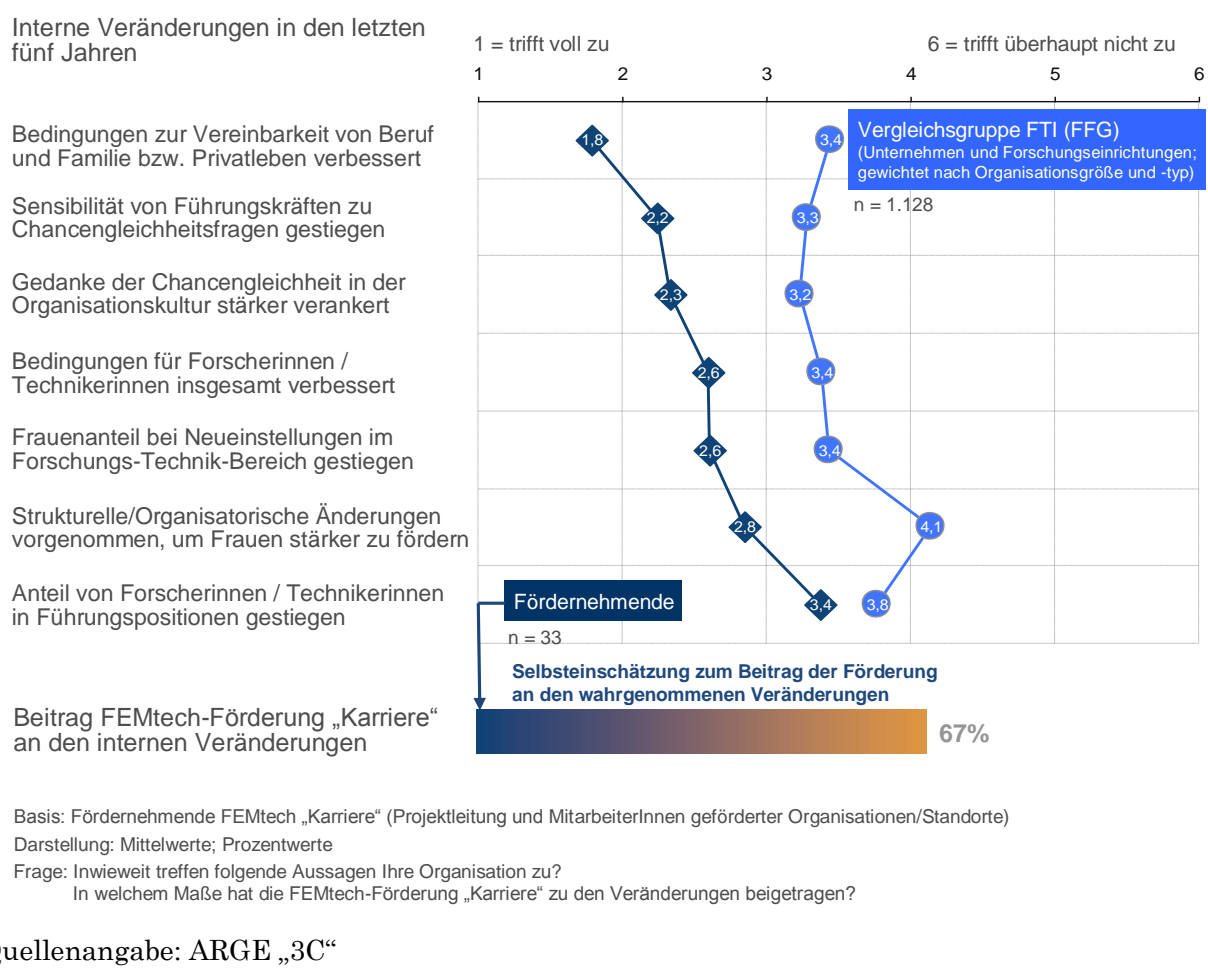

\section{Outcomes FEMtech Karrierewege}

Die Erreichung der Karrierewege-Förderziele weist Ähnlichkeiten mit FEMtech Karriere auf; vor allem hinsichtlich der Diskrepanz zwischen Förder- und Vergleichsgruppe und in deutlich geringerem Maße auch hinsichtlich der Unterschiede zwischen vorbereitenden Maßnahmen und tatsächlichem Niederschlag in erhöhtem Frauenanteil. Hierbei ist jedoch zu bedenken, dass die 
wahrgenommenen Veränderungen bezogen auf Kooperationen zwischen Unternehmen und Hochschulen allgemein abgefragt wurden, nicht in Bezug auf die eigene Organisation wie bei Karriere und FTI-Projekte.

Generell bewerten die Karrierewege-Geförderten wiederum - und auch dies ist nach eigenem Bekunden zu einem hohen Anteil von $66 \%$ auf die FEMtech Förderung zurückzuführen - alle Aspekte deutlich positiver als die Vergleichsgruppe. Letztere sieht kaum positive Veränderungen in der kooperativen (= im Rahmen gemeinsamer Projekte zwischen Unternehmen und Hochschulen) Förderung des weiblichen Nachwuchses.

Karrierewege-Fördernehmende sehen hingegen positive Veränderungen, allerdings eher in der verbesserten Angebotsschaffung zur Karrierevorbereitung von Studentinnen für die industrielle Forschung als bezogen auf die tatsächliche Entwicklung bei der Gewinnung von Praxis-Know-how und beim Berufseinstieg weiblicher Absolventinnen.

\section{Abbildung 37: Zielerreichung FEMtech Karrierewege}

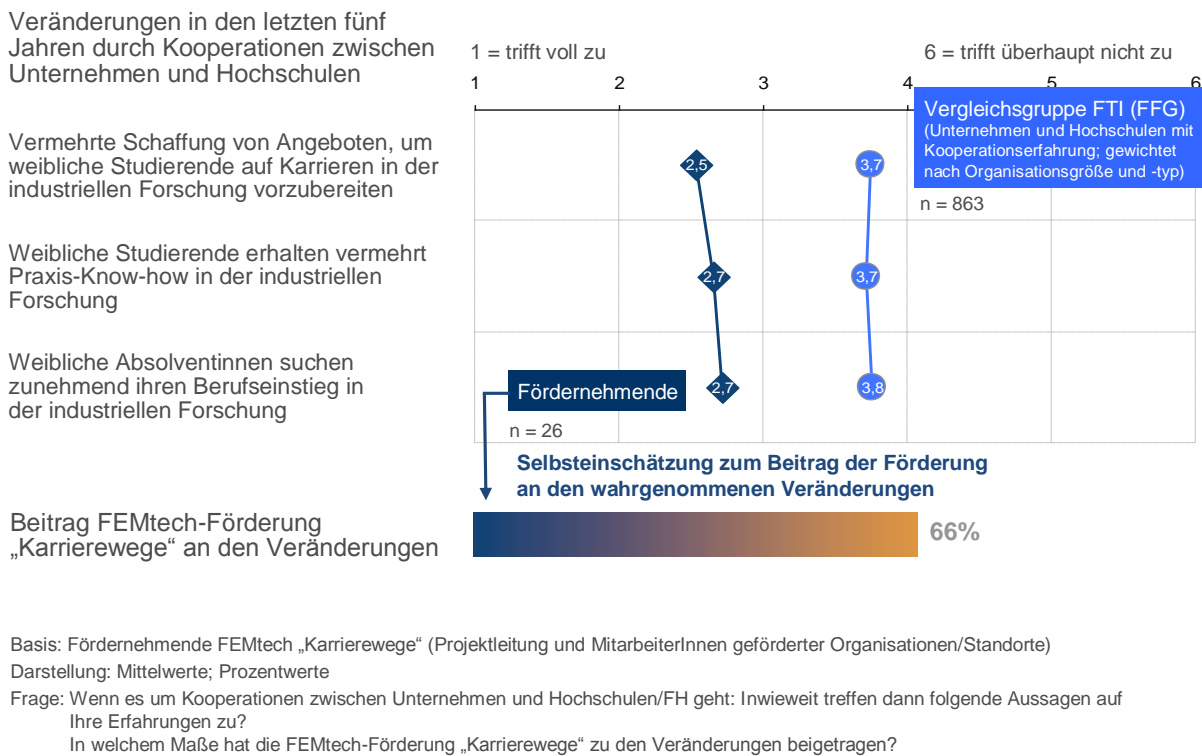

Quellenangabe: ARGE „3C“

\section{Outcomes FEMtech FTI-Projekte}

Auch FEMtech FTI-Projekte konnte durchaus Veränderungen im Sinne der intendierten Resultate erzielen, wie der Vergleich zwischen Fördernehmenden und Vergleichsgruppe zeigt.

Eine Bewusstseinsbildung in der Forschung zeigt sich in den geförderten Organisationen auf mehreren Ebenen: von der allgemeinen Berücksichtigung der Dimension „Geschlecht“ in Forschungsprojekten (Mw. 2,5) über die Zusammenstellung geschlechtergemischter Teams (Mw. 2,5) bis hin zur stärkeren Berücksichtigung der spezifischen Perspektive von Nutzerinnen in der Forschungsentwicklung (Mw. 2,6). Damit urteilen die FTI-Projekte-geförderten Organisationen - wie bei den beiden anderen Förderlinien auch - deutlich positiver als die jeweilige Vergleichsgruppe und sie rechnen diese Veränderungen wiederum zu einem hohen Anteil der FEMtech Förderung zu. Auffällig ist 
aber, dass sich die Effekte allesamt auf einem mittleren Niveau bewegen (Mw. zu den drei Aussagen von 2,5 bis 2,6) und dabei zugleich der Abstand zwischen Förder- und Vergleichsgruppe über alle Dimensionen hinweg geringer ausfällt als bei den anderen beiden Förderlinien.

Insgesamt betrachtet weist FTI-Projekte damit zwar durchaus Wirkung auf, jedoch scheint diese im direkten Vergleich mit den anderen beiden Förderlinien geringer auszufallen und sich weniger auf konkret strukturelle und faktische Veränderungen als allein auf die Bewusstseinsebene zu beziehen (was allerdings auch der Zielsetzung entspricht).

\section{Abbildung 38: Zielerreichung FEMtech FTI-Projekte}

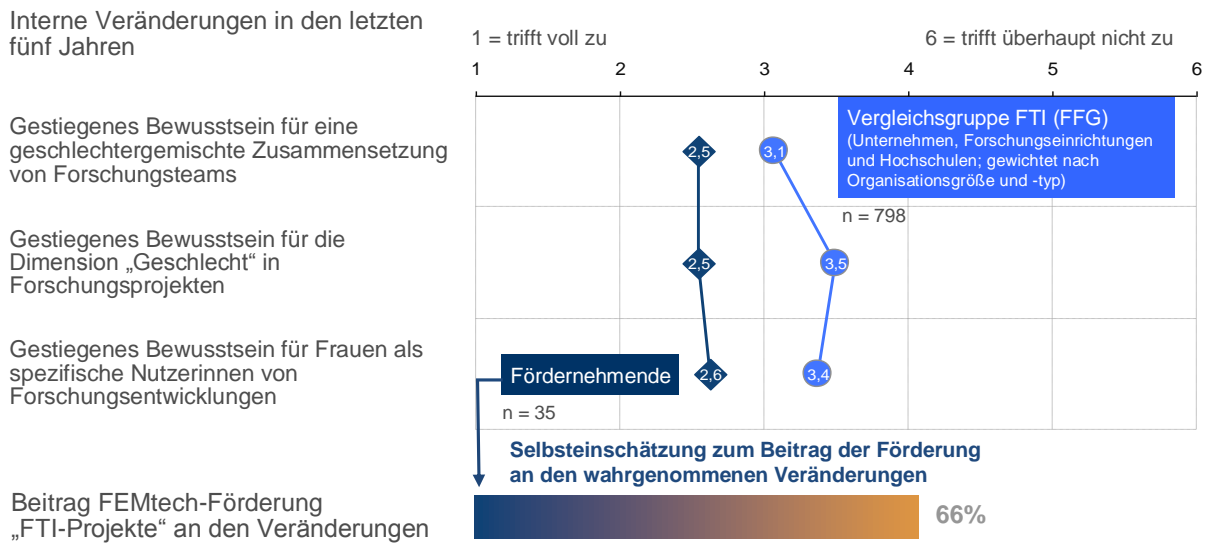

Basis: Fördernehmende FEMtech „FTI-Projekte“ (Projektleitung und Mitarbeiterlnnen geförderter Organisationen/Standorte) Darstellung: Mittelwerte; Prozentwerte

Frage: Inwieweit treffen folgende Aussagen auf Forschungs- und Entwicklungsprojekte Ihrer Organisation zu? In welchem Maße hat die FEMtech-Förderung „FTI-Projekte“ zu den Veränderungen beigetragen?

Quellenangabe: ARGE „3C“

\subsubsection{FEMtech Wirkungen: Aktivitäten}

Um Outcomes der FEMtech Aktivitäten in ihrer Gesamtheit zu beurteilen, stellen wir den NutzerInnen von FEMtech Aktivitäten zwei Vergleichsgruppen gegenüber, bei denen wir zwischen zwei unterschiedlichen Formen der NichtNutzung differenzieren: Vergleichsgruppe 1 kennt FEMtech zumindest dem Namen nach, nutzt aber keinerlei Angebote, während Vergleichsgruppe 2 FEMtech überhaupt nicht kennt.

Generell zeigt sich wie bereits bei Förderungen auch hier, dass die NutzerInnen in Bezug auf alle Zieldimensionen positiver urteilen - jedoch ist die Diskrepanz zwischen den Gruppen hier deutlich geringer als bei den Förderungen.

So sehen sowohl Aktivitäten-NutzerInnen als auch Vergleichsgruppe 1 (FEMtech namentlich bekannt), dass die öffentliche Diskussion zu Chancengleichheit und Frauen in FTI innerhalb der letzten fünf Jahre deutlich „befeuert“ und die öffentliche Präsenz erfolgreicher Frauen gestärkt wurde, die Vernetzung zum Thema dagegen deutlich weniger befördert wurde.

Dass Vergleichsgruppe 1 die konstatierten Entwicklungen, wenn auch auf etwas geringerem Niveau, ebenfalls sieht, spricht für eine indirekte Wirkung auch bei denen, die sich nicht vertieft mit FEMtech auseinandersetzen, aber 
zumindest von der Existenz des Programms wissen, den Namen kennen und „am Rande gestreift“ worden sind. Denn: Mit Awareness-Maßnahmen wie Auszeichnungen (z. B. der Gender Award 2010) oder „Expertin des Monats“ schafft FEMtech Anlässe für Medienberichterstattung und trägt somit zur Themen-Wahrnehmung bei, ohne dass RezipientInnen den Programmnamen FEMtech zwangsläufig als Absender erkennen müssen. Im Sinne einer „UBoot-Taktik" werden so vielmehr auch Gruppen erreicht, die sich ansonsten durch FEMtech wenig angesprochen fühlen und dementsprechend auch nicht selbst aktiv Angebote nutzen. Diese These einer ,indirekten Wirkung“ wird zudem dadurch gestützt, dass das Niveau der Einschätzungen in Vergleichsgruppe 1 insgesamt bereits im positiven Bereich liegt: Die Einschätzungen bewegen sich im Mittel zwischen 2,5 und 3,1 und sind damit deutlich besser als das Niveau der Vergleichsgruppen bei den Förderungen.

Ein ähnlicher Effekt einer ,indirekten“ und damit tatsächlich breiteren Öffentlichkeitswirkung lässt sich in Vergleichsgruppe 2 nur ansatzweise erkennen: Auch wenn diese eine stärkere öffentliche Diskussion zu „Chancengleichheit“ und „Frauen in FTI“ mit 2,9 im Mittel nur sehr bedingt wahrnehmen, so ist es insgesamt über alle Outcome-Fragen hinweg der beste Wert. Insofern haben auch Befragte der Online-Befragung, die FEMtech überhaupt nicht kennen, hier in Ansätzen Veränderungen im öffentlichen Diskurs bemerkt.

Die insgesamt recht großen Unterschiede zwischen den beiden Vergleichsgruppen verweisen auf die hohe Bedeutung von allgemeinen AwarenessAktivitäten: Denn ganz offensichtlich trägt bereits die reine Wahrnehmung „da gibt es ein Programm namens FEMtech mit zahlreichen Angeboten“ dazu bei, das Thema zu setzen und für dessen Bedeutung zu sensibilisieren. Auch wenn bei Vergleichsgruppe 1 keine vertiefte Auseinandersetzung mit Programminhalten und Angeboten erfolgt, sorgt ein permanentes öffentliches Grundrauschen bei dieser Gruppe bereits für Effekte.

FEMtech ist selbstverständlich nicht alleinige Absenderin entsprechender Botschaften, sodass die bemerkten Verbesserungen in Bezug auf die öffentliche Diskussion und Präsenz von Frauen in FTI nicht allein dem Programm zugerechnet werden können. Nach Einschätzung der FEMtech AktivitätenNutzerInnen hat FEMtech jedoch mit knapp $50 \%$ einen großen Anteil daran. Angesichts des Charakters der Maßnahmen erstaunt es nicht, dass der Anteil bei Aktivitäten geringer eingeschätzt wird als bei Förderungen, die unmittelbarer in den Organisationen wirken können (vgl. Kapitel 4.3.1 „Outcomes FEMtech Förderungen“).

Dass bezüglich der themenspezifischen Vernetzung geringere Verbesserungen gesehen werden, könnte laut FTI-politischen AkteurInnen (und dieser Eindruck wird durch offene Ergänzungen in der Online-Befragung bestätigt) am selbstreferenziellen Charakter von FEMtech Veranstaltungen liegen. Ihrer Wahrnehmung nach trifft sich insbesondere auf den Netzwerktreffen ein „eingeschworener Kreis“ bzw. „eine enge Community“, die die Ziele von FEMtech bereits seit Jahren unterstützt und zum großen Teil selbst in das Programm involviert ist oder von seinen Angeboten profitiert (etwa als registrierte Expertin in der Expertinnen-Datenbank). Demnach überrascht es auch nicht, dass gerade die auf den Netzwerktreffen unterrepräsentierten Privatunternehmen aus FTI (vgl. Kapitel 3.2.3 „Nutzung und erreichte Zielgruppen je Aktivität“ - 
insbesondere Tabelle 17: Zwar stellen Unternehmen mit 32 \% die größte Gruppe der TeilnehmerInnen dar; gemessen an der Grundgesamtheit von Unternehmen in Österreich ist ihr Anteil an den TeilnehmerInnen damit jedoch gering - insbesondere, wenn gleichzeitig $27 \%$ aus außeruniversitären Forschungseinrichtungen und zusammengenommen $34 \%$ aus Hochschulen kommen) den Beitrag von FEMtech zur Vernetzung als eher moderat einschätzen (Mw. 3,3).

Eine Erweiterung des Publikums bei den Netzwerktreffen wird zusätzlich durch die starke Konzentration dieser Veranstaltungen auf Wien erschwert. Dementsprechend wird sowohl seitens FTI-politischer AkteurInnen als auch von zahlreichen Befragten in der Online-Befragung eine Ausweitung der Netzwerktreffen in weitere Bundesländer angeregt.

\section{Abbildung 39: Zielerreichung FEMtech Aktivitäten}

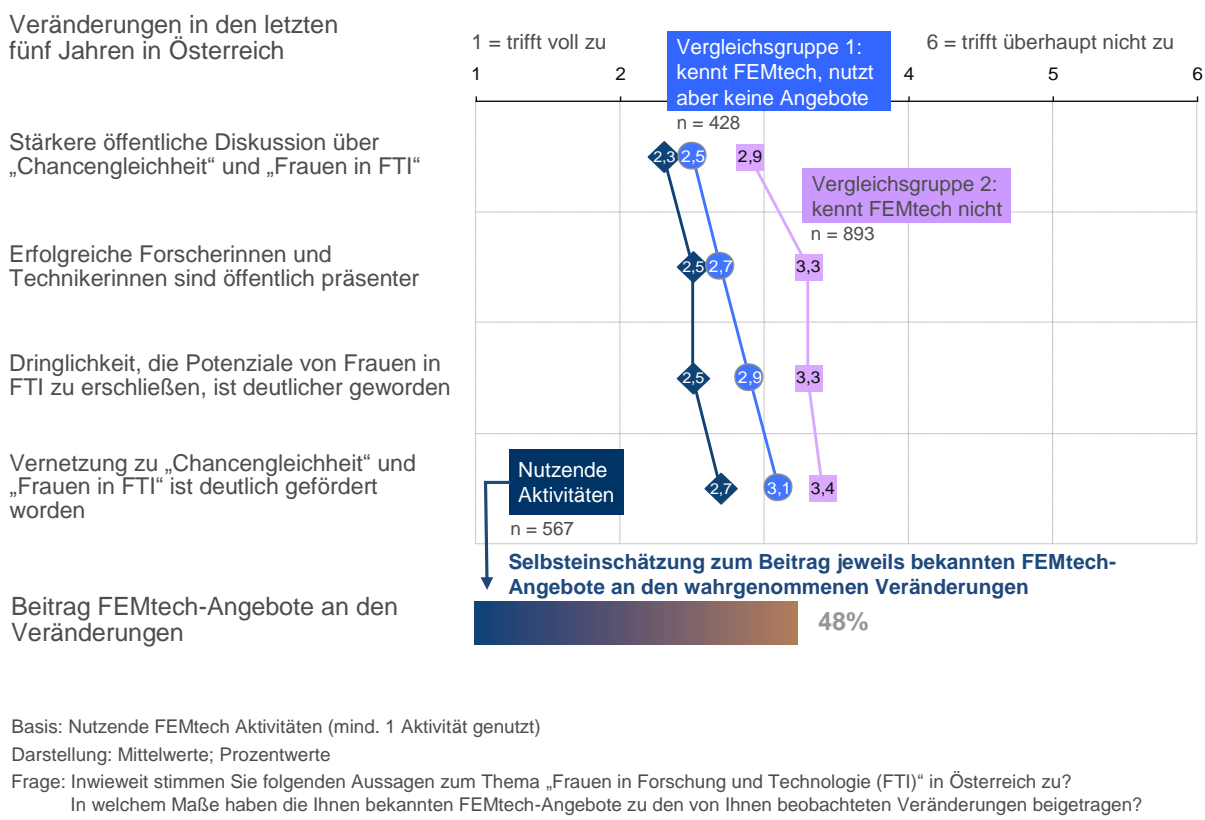

Quellenangabe: ARGE „3C“

\subsubsection{FEMtech Wirkungen: Wissen}

Auch bei „Wissen“ zeigt sich bezogen auf die Zielerreichung vom Grundsatz her die gleiche Tendenz wie bei „Förderungen“ und „Aktivitäten“: NutzerInnen der Wissens-Angebote urteilen in Bezug auf die abgefragten Zieldimensionen deutlich positiver als Nicht-NutzerInnen- und rechnen die bemerkten Verbesserungen ebenfalls zu einem hohen Anteil (56 \%) FEMtech zu.

Mit den unter „Wissen“ gebündelten Informationsangeboten ist es FEMtech insbesondere gelungen, das Thema der Chancengleichheit in Forschung und Technologie mit Fakten zu hinterlegen. Dies bestätigen nicht nur NutzerInnen der Wissensangebote, die ein deutlich verbessertes Angebot diesbezüglich konstatieren, sondern auch die interviewten FTI-politischen AkteurInnen wobei Letztere sogar bereits ein „Zuviel“ des Angebots monieren. 
Während damit das Ziel der Wissensbereitstellung weitestgehend erfüllt wurde, fällt das Urteil hinsichtlich der tatsächlichen Verbesserung des organisationsinternen Wissens bereits etwas verhaltener aus: So hat bei NutzerInnen der Wissensangebote FEMtech zwar durchaus zu einer soliden Verbesserung interner Kenntnisse beigetragen (Mittelwert 2,6) und in der Praxis werden diese Informationen - das zeigen offene Ergänzungen in der Online-Befragung - durchaus auch verwendet, v. a. im organisationsinternen Diskurs als Argumentationshilfe, um im Sinne eines „reality checks“ Karrierehürden für Frauen aufzeigen zu können.

Die Effekte bleiben aber weitgehend auf einen engen Kreis beschränkt, der die gesamte Palette von FEMtech Angeboten bereits intensiv nutzt (mind. 7 genutzte Angebote) oder Förderung erhält. Beide Vergleichsgruppen sehen dementsprechende Veränderungen auf einem deutlich schwächeren Niveau. Der in Bezug auf die Aktivitäten (siehe vorangegangenes Kapitel) beschriebene Effekt einer „indirekten Wirkung“ insbesondere bei Vergleichsgruppe 1 (kennen FEMtech namentlich, nutzen aber keine Angebote) zeigt sich in Bezug auf Wissen in deutlich geringerem Maße: Zwar wertet diese Gruppe auch hier deutlich besser als Vergleichsgruppe 2 (kennt FEMtech überhaupt nicht), jedoch ist der Unterschied zwischen den Gruppen nicht so hoch wie etwa der Unterschied zwischen NutzerInnen und Vergleichsgruppe 1.

FTI-politische AkteurInnen weisen zudem darauf hin, dass speziell das Gender Booklet stark auf die außeruniversitäre naturwissenschaftlich-technische Forschung zugeschnitten ist und deshalb anderen FEMtech Zielgruppen kaum Orientierung bietet. Darüber hinaus erschwere die über die Jahre gewachsene Fülle an nach verschiedenen Segmenten und Subsektoren ausdifferenzierten Statistiken insbesondere Personen, die FEMtech nur sporadisch nutzen, eine schnelle Orientierung.

Abbildung 40: Zielerreichung FEMtech Wissensangebote

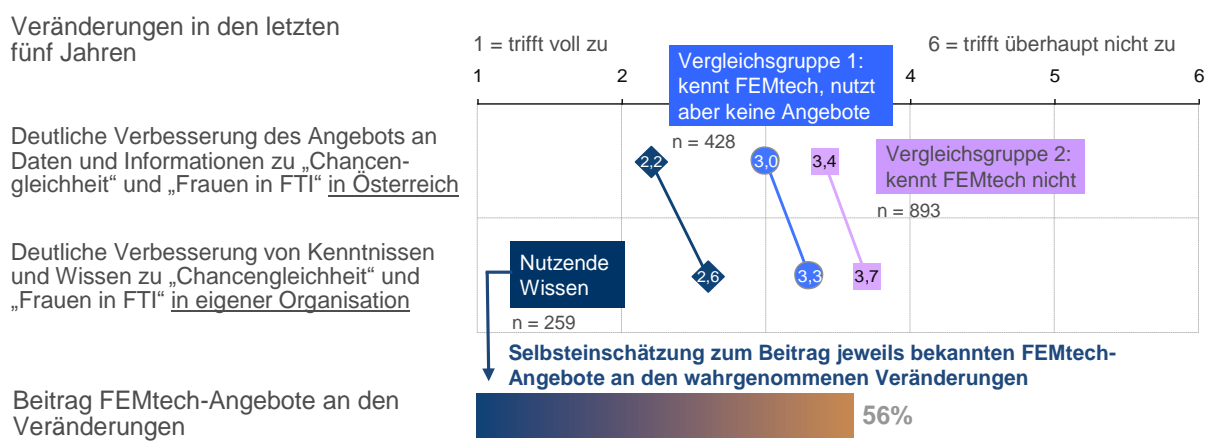

Basis: Nutzende FEMtech Wissensangebote (mind. 1 Wissensangebot genutzt)

Darstellung: Mittelwerte; Prozentwerte

Frage: Inwieweit stimmen Sie folgenden Aussagen zum Thema „Frauen in Forschung und Technologie (FTI)“ in Österreich zu? Und inwieweit stimmen Sie folgender Aussage in Bezug auf Ihre eigene Organisation zu.

In welchem Maße haben die Ihnen bekannten FEMtech-Angebote zu den von Ihnen beobachteten Veränderungen beigetragen?

Quellenangabe: ARGE „3C“ 


\subsection{4 Übergreifende Wirkungen aus Sicht geförderter Frauen}

Zahlreiche Forscherinnen und Technikerinnen, seien es Berufseinsteigerinnen oder erfahrene Expertinnen, haben über unterschiedliche Schienen von FEMtech Angeboten direkt oder indirekt beruflich und/oder persönlich profitiert. Dazu gehören unmittelbar involvierte Frauen in den über die Förderlinie geförderten Organisationen ebenso wie zum Beispiel „Expertinnen des Monats“.

In den qualitativen Fokus-Interviews hebt diese Zielgruppe vor allem zwei positive Veränderungen hervor, die sich durch FEMtech für sie ergeben haben: Zum einen eine Erweiterung des persönlichen Netzwerks; zum anderen eine gestiegene positive Aufmerksamkeit des Umfelds: „Frauen vernetzen sich stärker, werden selbstbewusster, werden auf Vorträge und Seminare eingeladen. Nach dem Projekt wurde auch ein Zeitungsartikel veröffentlicht, auf den viele positive Reaktionen kamen " (Zitat FN1). Strukturell-organisatorische Veränderungen (etwa Aufstieg in höhere Positionen) gab es eher nicht.

FEMtech hat aus Sicht der Frauen dazu beigetragen, in den geförderten Organisationen mehr Selbstverständlichkeit in das Thema "Chancengleichheit" zu bringen (,man wird nicht mehr schief angeschaut oder belächelt" (Zitat FN1)). So werde z. B. der Aspekt Work-Life-Balance zunehmend ein selbstverständlicher Bestandteil in MitarbeiterInnengesprächen, als Mitarbeiterin sei man nicht mehr „Bittstellerin“, sondern es werde gemeinsam an Lösungen gearbeitet.

Die Effekte von FEMtech vollziehen sich demnach vor allem auf der Ebene der Bewusstseinsbildung und Sensibilisierung - sowohl bei den Frauen als auch in deren beruflichem Umfeld. Dementsprechend gilt der „Awareness“-Ansatz von FEMtech, der auf breitenwirksame Aktionen ebenso setzt wie auf den Aufbau einer Community, unter den Frauen als enorm wichtig.

\subsection{Conclusio: FEMtech Wirkungsketten}

Um abschließend die Wirkungen von FEMtech zu beurteilen, werden nachfolgend die Erkenntnisse aus der Portfolioanalyse (vgl. Kapitel 3 „FEMtech Portfolio-Analyse") und aus diesem Kapitel zusammengebracht und in grafischer Form als Wirkungsketten dargestellt. Dabei sei einmal mehr darauf verwiesen, dass die Analyse der Outcomes sich aus den genannten Gründen allein auf wahrgenommene Veränderungen bei den Zielgruppen stützen muss.

Die FEMtech Förderungen erreichen ihre Ziele innerhalb der geförderten Organisationen je nach Zieldimension in unterschiedlichem Ausmaß. Ihnen ist gemeinsam, dass sie ihre Wirkung vor allem in solchen Organisationen entfalten, in denen bereits vorab eine entsprechende Affinität und Sensibilisierung herrschte. In erster Linie konnten für die Umsetzung konkreter Maßnahmen also Unternehmen erreicht werden, in welchen bereits ein Grundverständnis für die Thematik vorhanden war. In der Breite wurden die FTI-Unternehmen der privaten Wirtschaft nicht umfassend erreicht, im Vergleich zur Gesamtheit des FFG-Förderangebots.

„Karriere“ erzielt gute Effekte bezogen auf eher weiche Faktoren der Bewusstseinsbildung und Sensibilisierung in Richtung Chancengleichheit. Struk- 
turelle und organisatorische Veränderungen umfassen vor allem Maßnahmen zur Verbesserung einer Work-Life-Balance (de facto in erster Linie Flexibilisierung von Arbeitszeiten), systematischerem und gendersensibilisierten Personalrekrutierung und -entwicklung (letzteres nur in Großunternehmen), in geringem Umfang solche einer expliziten Förderung von Frauen. Der Niederschlag in konkreten Zahlen (Anstieg des Frauenanteils bei Neueinstellungen und Führungspositionen) ist laut Onlinebefragung (vgl. auch Kapitel 4.3.1 „Outcomes FEMtech Förderungen“ - insb. Abbildung 36) nicht eindeutig nachweisbar.

Bei „Karriere“ wird zudem besonders deutlich, dass die Förderung vor allem zu einer Dynamisierung geführt hat: Ohnehin geplante Maßnahmen wurden schneller, umfassender und systematischer umgesetzt. Es werden also weniger Unternehmen und Organisationen neu angeregt, sich zum Thema Chancengleichheit zu engagieren als vielmehr in ihrem Tun bestärkt und unterstützt. Als weitere Einschränkung kommt hinzu, dass das Antragsprozedere und die anstehenden Berichtpflichten abschreckend auf „Ungeübte“ wirken und aus diesem Grund oft keine Förderung beantragt wird. Der Kreis der Einreichenden schränkt sich also ein, die eingehenden Anträge sind zudem wenig innovativ und beinhalten vorrangig Standardmaßnahmen.

Abbildung 41: Wirkungskette FEMtech Karriere

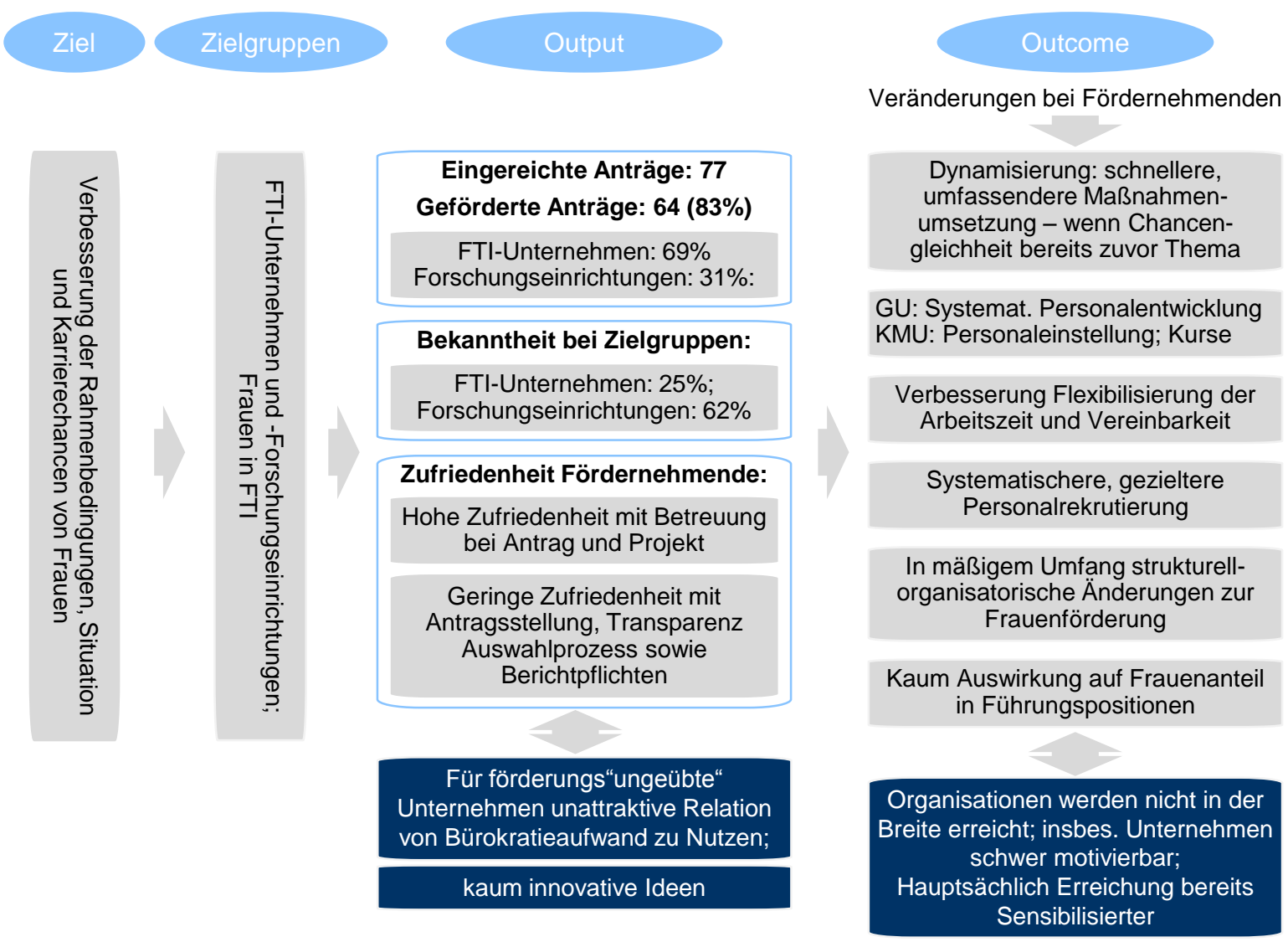

Quellen: Portfolio-Analyse, Online-Befragung FEMtech-AdressatInnen, -Fördernehmende und FTI-Unternehmen und Organisationen (FFG); Interviews mit oberem Management aus FTI-Unternehmen, FTI-politischen Akteuren, FEMtechgeförderten Frauen, ehemalig. Programmleiterinnen, Analyse der FEMtech-Medienresonanz 
„Karrierewege“ trägt zu einer Angebotsausweitung und Systematisierung der Zusammenarbeit von Unternehmen und Hochschulen in Fragen der weiblichen Nachwuchsgewinnung bei. Da bei der Umsetzung jedoch letztendlich einzelne Personen gefördert werden (durch die Förderung von Praktika, Diplomarbeiten, Dissertationen in Kooperationen sowie begleitende Angebote für potenzielle weibliche Nachwuchskräfte), sind keine strukturellen Effekte zu erwarten. Der tatsächliche Niederschlag in Form von mehr weiblichen Berufseinsteigerinnen in die Industrie ist damit ebenfalls schwer abschätzbar.

Karrierewege wurde als „Pilotprojekt“ durchgeführt, die Weiterführung ist mit Ende der Evaluation noch nicht entschieden.

Abbildung 42: Wirkungskette FEMtech Karrierewege

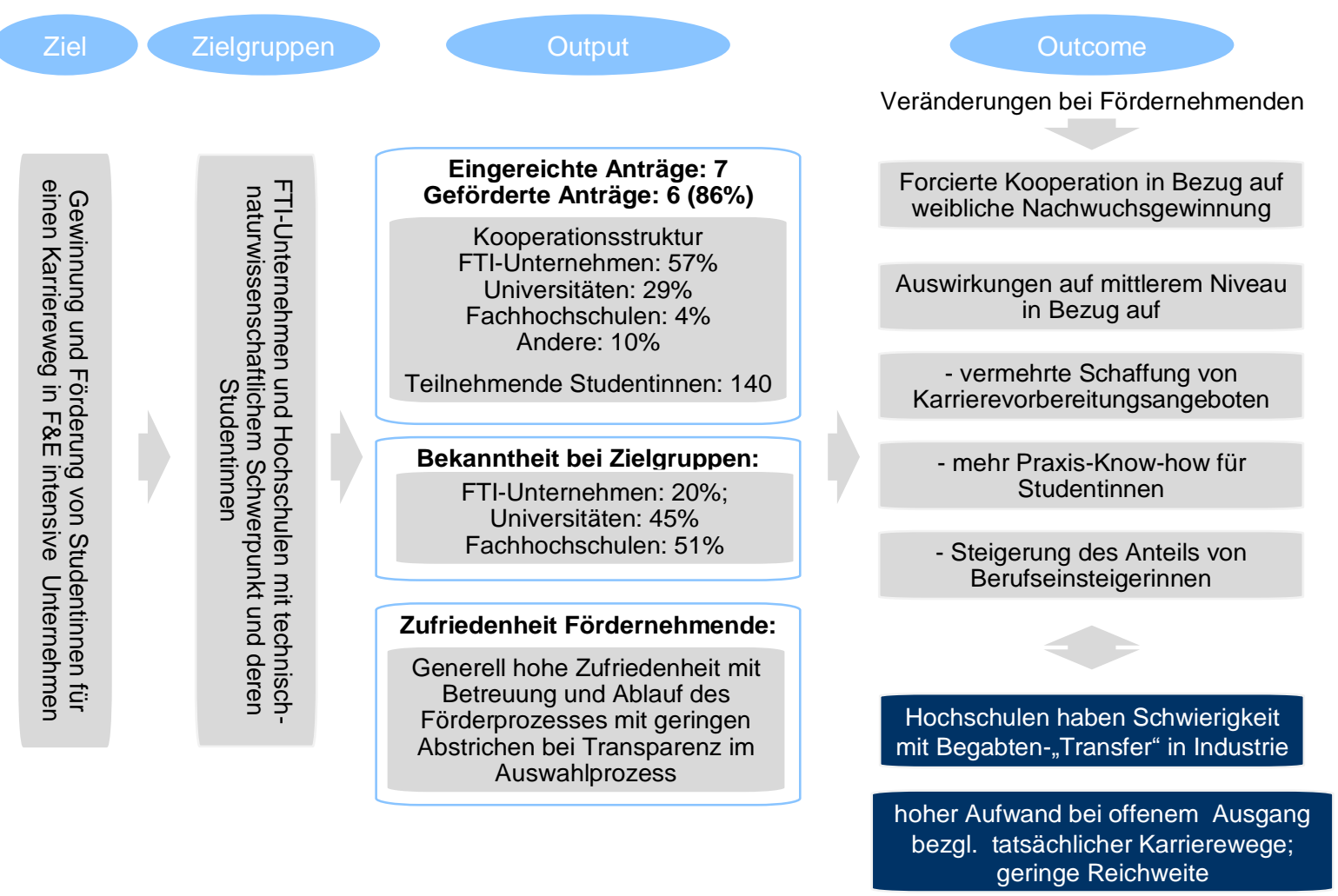

Quellen: Portfolio-Analyse, Online-Befragung FEMtech-AdressatInnen, -Fördernehmenden und FTI-Unternehmen und Organisationen (FFG); Interviews mit oberem Management aus FTI-Unternehmen, FTI-politischen Akteuren, FEMtechgeförderten Frauen, ehemalig. Programmleiterinnen, Analyse der FEMtech-Medienresonanz

„FTI-Projekte“ hat als einzige Förderlinie eine Einreichquote, die deutlich über den tatsächlich bewilligten Projekten liegt. Die Förderlinie erzielt auf den verschiedenen Ebenen der Bewusstseinsbildung zur Genderdimension in Forschungsprojekten durchgängig eine Wirkung auf „mittlerem“ Niveau. Im Vergleich mit „Karriere“, die als Förderlinie auch unmittelbar organisationsintern wirken soll, fallen die erzielten Veränderungen jedoch unspezifischer aus. Das sehr breite Themenspektrum vermittelt den Eindruck, dass sich diese Förderlinie noch im „Experimentierstadium“ befindet. Hinzu kommt, dass die Zielgruppe Unternehmen für das Thema "Gender in Forschungsprojekten“ erst wenig sensibilisiert zu sein scheint: Sie beteiligen sich in geringerem Ausmaß als bei den beiden anderen Förderlinien; statt dessen werden Universitäten 
erreicht, die aufgrund bis dato fehlender Förderlinien für zum Teil bereits bestehende Projektideen eine starke Motivation zur Einreichung aufweisen.

Abbildung 43: Wirkungskette FEMtech FTI-Projekte

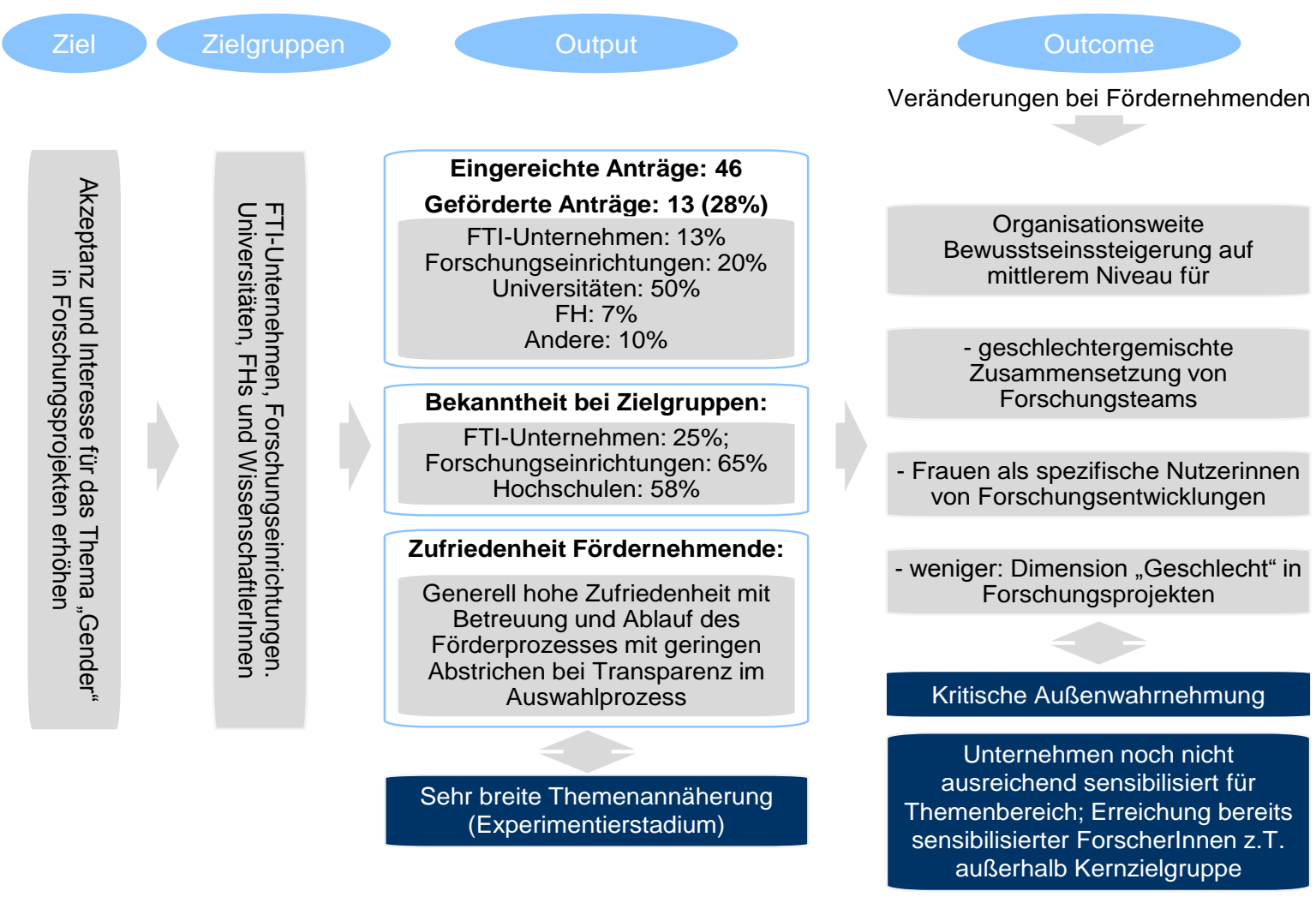

Quellen: Portfolio-Analyse, Online-Befragung FEMtech-AdressatInnen, -Fördernehmenden und FTI-Unternehmen und Organisationen (FFG); Interviews mit oberem Management aus FTI-Unternehmen, FTI-politischen Akteuren, FEMtechgeförderten Frauen, ehemalig. Programmleiterinnen, Analyse der FEMtech-Medienresonanz

Mit den unter „Aktivitäten“ gebündelten Formaten zur Sensibilisierung, Bewusstseinsbildung und Vernetzungsförderung in Kombination mit Presse- und Öffentlichkeitsarbeit sorgt FEMtech für eine kontinuierliche Präsenz des Themas im öffentlichen Diskurs. Und in der Tat ist der Bekanntheitsgrad, den sich FEMtech in der FTI-Unternehmens- und Organisationslandschaft generell also auch jenseits des regelmäßig informierten engeren AdressatInnen-Kreises - erarbeitet hat, mit $44 \%$ beachtlich.

FEMtech hat seine Ziele erreicht, die öffentliche Diskussion zu „Chancengleichheit" und „Frauen in FTI“ zu stärken. Damit hat FEMtech - nicht allein, sondern zusammen mit anderen Programmen und AbsenderInnen - einen wichtigen Beitrag zum Agenda-Setting geleistet. „Put the issue on the map: Das Thema wird nicht mehr ignoriert" (Zitat FTI-pA5). In eingeschränktem Umfang wurde auch das Ziel der Vernetzung erreicht; hier beschränkt sich die Wirkung aber auf eine enge, per se stark themenaffine Community.

Bezogen auf „Aktivitäten“ insgesamt gilt jedoch ähnlich wie bei „Förderungen“, dass privatwirtschaftliche Unternehmen in der Breite in geringerem Umfang erreicht wurden und diese zudem weniger zufrieden mit den „Aktivitäten“ sind als Forschungseinrichtungen und Hochschulen. 
Auch wenn eine Differenzierung der Beiträge einzelner Aktivitäten zur Wirkung des gesamten Maßnahmenbündels nicht möglich ist, hat die PortfolioAnalyse (vgl. Kapitel 3 „FEMtech Portfolio-Analyse“) dennoch klare Tendenzen zum Funktionieren einzelner Formate und damit ihrer Rolle im Gesamtset aufzeigen können.

Abbildung 44: Wirkungskette FEMtech Aktivitäten
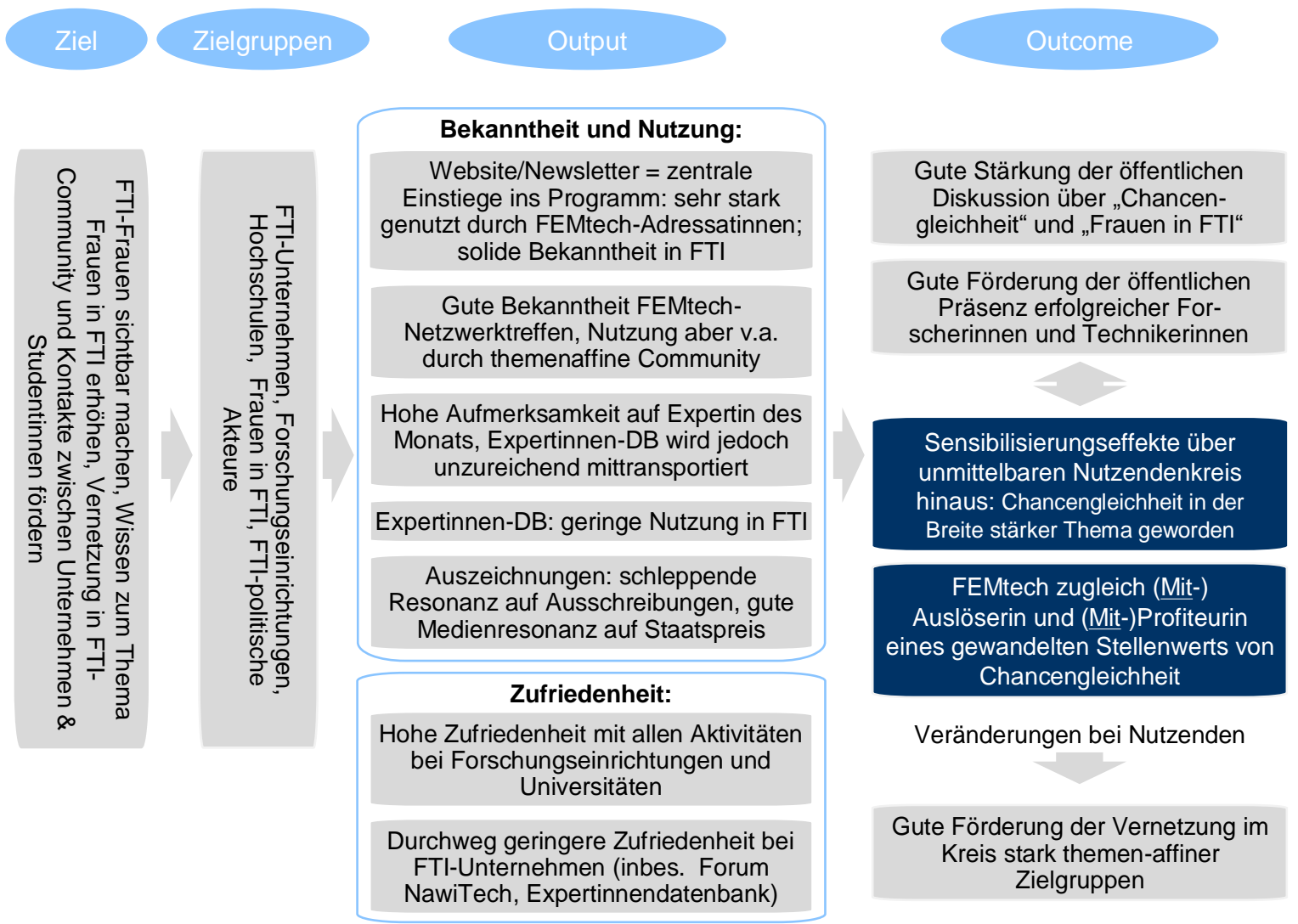

Sensibilisierungseffekte über

unmittelbaren Nutzendenkreis

hinaus: Chancengleichheit in der

Breite stärker Thema geworden

Quellen: Portfolio-Analyse, Online-Befragung FEMtech-AdressatInnen, -Fördernehmenden und FTI-

Unternehmen und Organisationen (FFG); Interviews mit oberem Management aus FTI-Unternehmen, FTI-

politischen Akteuren, FEMtech-geförderten Frauen, Analyse der FEMtech-Medienresonanz

Bei „Wissen“ beschränkt sich die Wirkung stark auf die engere „FEMtech Community“, die die diesbezüglichen Angebote intensiv nutzt und dementsprechend ein deutlich verbessertes Angebot an Daten und Fakten wahrnimmt. In den Organisationen der NutzerInnen hat sich nach deren Wahrnehmung auch der interne Wissensstand auf mittlerem Niveau verbessert. Die Zielgruppe der Unternehmen wird mit „Wissen“ nur marginal erreicht; zudem treffen die Angebote zu wenig deren Bedürfnislage und sind auf der Website zudem zu wenig zielgruppenspezifisch aufbereitet. 
Abbildung 45: Wirkungskette FEMtech Wissen

\section{Zie}

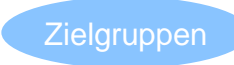

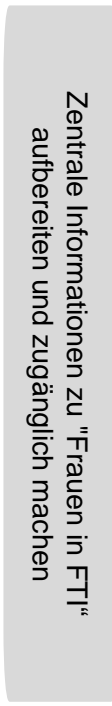

Output

\section{Bekanntheit und Nutzung:}

Wissensangebote sind v.a. in engerer "FEMtech-Community" bekannt, werden dort gezielt und umfassend genutzt (Ausnahme: gender diskurs)

In Breite der FTI-Landschaft sehr geringe Bekanntheit und Nutzung

\section{Zufriedenheit: \\ Sehr hohe Zufriedenheit in engerer Community}

Geringere Zufriedenheit bei FTIUnternehmen

Daten: Überangebot; Publikationen: zu starker Fokus auf eigenen Maßnahmen

Aufbereitung auf Website zu wenig strukturiert und zielgruppenspezifisch

Entspricht zu wenig Unternehmensbedarfen

\section{Outcome}

Veränderungen bei Nutzenden

Guter Beitrag zur Verbesserung des Angebots an Daten/Informationen zu

"Chancengleichheit" und „Frauen in FTI" in Österreich

Hilfreich als organisationsinterne Argumentationshilfe

Solide Verbesserung interne

Kenntnisse und Wissen zu

"Chancengleichheit" und „Frauen in FTI"

\section{Keine Breitenwirkung:}

Effekte beschränkt

auf engen Nutzendenkreis

Quellen: Portfolio-Analyse, Online-Befragung FEMtech-AdressatInnen, -Fördernehmenden und FTI-

Unternehmen und Organisationen (FFG); Interviews mit oberem Management aus FTI-Unternehmen, FTI-

politischen Akteuren, FEMtech-geförderten Frauen, Analyse der FEMtech-Medienresonanz 


\section{Performance \& Embedding}

\subsection{Programmentwicklung}

Zusammenfassende Analysen zur FEMtech Programmentwicklung basieren auf Programmdokumenten, den Programmgesprächen mit BMVIT, FFG und den Organisationen des Kompetenzzentrums, eines gemeinsamen „ProgrammHistorien“-Gesprächs mit BMVIT, ÖGUT, Joanneum Research und FFG, Fokusinterviews mit ehemaligen Programmleiterinnen sowie den SelfAssessments der Programm-UmsetzerInnen.

Im August 2003 wurde das Programm „FEMtech - Frauen in Forschung und Technologie“ gestartet. Vorangegangen war eine Ausschreibung des BMVIT zur Umsetzung des neuen Programms im Rahmen der Forschungsförderung. Als Sieger des Wettbewerbes ging die Technologie Impulse Gesellschaft (Vorläuferorganisation der FFG) in einer Projektpartnerschaft mit Joanneum Research und D\&Z Consulting hervor (die ÖGUT war zu diesem Zeitraum ein Werkvertragspartner des BMVIT und in die Programmumsetzung mit Teilaspekten eingebunden). Dieses Konsortium wurde gewählt, da mit der Ausschreibung bereits klar war, dass dies kein „Standard“-Forschungsförderungsprogramm sein soll, sondern vielfältige Aktivierungs- und Sensibilisierungsmaßnahmen stattfinden sollen. Eine Bandbreite von Forschung, Unternehmensberatung, FTI-Genderexpertise und Programmumsetzung war dazu gefragt.

FEMtech startete operativ 2004 mit einem Angebot aus mehreren Förderlinien, mit internen Studien und den Netzwerktreffen. Bereits für Frauenförderung und Chancengleichheit sensibilisierte FTI-Unternehmen und Forschungseinrichtungen reichten rasch die ersten Projekte in der Förderlinie FEMtech Karriere ein und begannen mit der Umsetzung.

Folgende FEMtech Förderlinien gab es seit 2004:

- FEMtech Karriere: Die einzige durchgehende Förderlinie, die zudem durch eine externe Akquisition begleitet wird und sich zuerst ausschließlich an Unternehmen, bald jedoch auch an außeruniversitäre Forschungseinrichtungen (nach Beendigung der speziellen Förderlinie) richtete.

- FEMtech FH: Ein Angebot an Fachhochschulen zur Erweiterung ihrer Chancengleichheitsaktivitäten (in Ergänzung der Förderaktivitäten des BMVIT mit dem FHplus-Programm). Hier erfolgte ein einmaliger Call im Jahr 2004. Es wurde noch ein zweiter Call angedacht, jedoch nicht durchgeführt.

- FEMtech Forschungsunternehmen: Dies war ursprünglich die Förderschiene für die außeruniversitäre Forschung, wurde jedoch bald in die Förderlinie FEMtech Karriere integriert.

- Eine Förderkombination mit dem FWF-Programm „Forscher für die Wirtschaft“" wurde 2004 angedacht, konnte aus förderrechtlichen Gründen jedoch nicht umgesetzt werden. 
- Mitte 2008 starteten die beiden Förderlinien FEMtech Karrierewege und FEMtech FTI-Projekte, die beide per Call abgewickelt werden.

FEMtech hatte in der Startphase einen weiteren Schwerpunkt: FEMtech Technologieprogramme. Hierbei ging es um die Sensibilisierung FTIpolitischer AkteurInnen und der Forschungsförderung in Bezug auf Frauenförderung und Chancengleichheit. Diese Leistungen blieben bis dato Bestandteil des FEMtech Portfolios, werden jedoch nicht mehr dezidiert als eigener Schwerpunkt geführt.

Nach über siebenjähriger Laufzeit finden sich zum Evaluationszeitpunkt bei FEMtech Förderungen folgende drei Programmlinien: FEMtech Karriere, FEMtech Karrierewege und FEMtech FTI-Projekte.

(Übersicht: siehe Abbildung 46 auf der nächsten Seite!) 
Abbildung 46: FEMtech Programmhistorie

\begin{tabular}{|c|c|c|c|c|c|c|c|c|c|c|c|c|}
\hline & FEMtech Historie & 2003 & Start: Juli 03 & 2004 & & 2005 & & 2006 & 2007 & 2008 & 2009 & 2010 \\
\hline \multirow{7}{*}{ 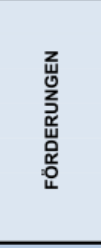 } & FEMtech Karriere & & & & & & $\pi$ & $\longrightarrow$ & & Verdopplung Fördernöhe & & \\
\hline & FEMtech Forschungunternehmen & & & & & & & 7 & & & & \\
\hline & FEMtech FH & & & & 1 Call & & & beendet & & & & \\
\hline & FEMtech Karrierewege & & & & & 8 & & & & 1. Call & 2. Call & Winterschool \\
\hline & FEMtech FTI-Projekte & & & & & & & & & 1. Call & 2. Call & 3. Call \\
\hline & Forscherinnen für die Wirtschaft (FWF) & & & & Idee - keine Realisienung & & & & & & & \\
\hline & externe Akquisition & & & & & & & & & & & \\
\hline \multirow{12}{*}{ 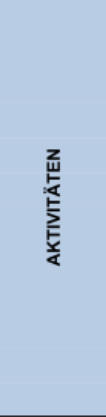 } & Expertin des Monats & & & & & seit 2005 & & & & & & \\
\hline & Expertinnen-Datenbank & & & & seit Mitte 04 & & & & & & & \\
\hline & Netzwerktreffen & & & & & & & & & & & \\
\hline & Forum NAWI-Tech & & & & & & & \multicolumn{2}{|c|}{ seit 06/2006 } & & & \\
\hline & FEMtech meets fiction & & & & & & & & & \multicolumn{2}{|c|}{ 1. Mal Nov.08 } & \\
\hline & Mentoring & & & & & & \multicolumn{2}{|c|}{ TU Wien Studie Pliotprojekt } & & & & GenderDiskurs \\
\hline & Gendercocktail & & & & & & Gender Coktail & Gender Coktail & Gender Coktail & Gender Coktail & Gender Coktail & Gender Coktail \\
\hline & Staatspreis für Chancengleichheit & & & & & & & & & & Sep.09 & \\
\hline & Auszeichnung & & & & & & & & & & & Mai.10 \\
\hline & Konferenz & & & & & & & & 15./16.5. & & & \\
\hline & Newsletter & & & & & & & & & & & \\
\hline & Website & & & & & & & & & & & \\
\hline \multirow{5}{*}{$\begin{array}{l}\text { W. } \\
\frac{\omega}{3}\end{array}$} & Gender Booklet & & & & Gender Booklet & & Gender Booklet & Gender Booklet & Gender Booklet & Gender Booklet & Gender Booklet & \\
\hline & genderDiskurs & & & & & & & & & & Jân.09 & \\
\hline & FEMtech Publikationen & & & & & & & & & & & \\
\hline & Daten & & & & & & & & & & & \\
\hline & Literatur & & & & & & & & & & & \\
\hline Programme & FEMtech Technologieprogramme & & & & & & & & & \begin{tabular}{|l|l} 
autgelassen \\
\end{tabular} & & \\
\hline \multirow{4}{*}{ 它 } & internationale Kontakte & & & & Sofia; EPWS & & & & & \multicolumn{2}{|c|}{ D-A-CH Austausch Konferenzzen } & \\
\hline & interne Wissensprojetke & & & & & & & & & & Gender-FTI & \\
\hline & Presse, Medien & & & & & & & FEMtech kulinarisch & FEMtech kulinarisch & & & \\
\hline & fFORTE & \multicolumn{5}{|c|}{ BMUK fFORTE academics \& FEMTech FFORTE, RFTE } & & W-FFORTE & \multicolumn{2}{|c|}{ FFORTE Schule } & & \\
\hline
\end{tabular}

Quellenangabe: ARGE „3C“ 
Die Programmlinien „Aktivitäten“ und „Wissen“ waren zu Beginn nicht differenziert - vielmehr galten alle „Nicht-Förderungen“ als Awarenessmaßnahmen. Als erstes starteten die FEMtech Netzwerke - insgesamt haben 27 Treffen seit 2003 stattgefunden. Die Website und der Newsletter boten von Anfang an Informationen für alle Interessierten. In den Anfangszeiten von FEMtech gab es vermehrt interne Studien, um das Know-how und die Datenbasis für das Programm aufzubauen. Der erste Gendercocktail beim Forum Alpbach fand sehr guten Anklang, sodass beschlossen wurde, diesen jährlich anzubieten. Mittlerweilen gehört er quasi zum Fixbestandteil des Alpbach-Programms und fungiert als eine Art „inoffizielle Eröffnung“.

Die FEMtech Programmentwicklungsphasen korrelieren stark mit der Organisationsstruktur von FEMtech. Insgesamt sind in den 7 Jahren drei Phasen zu unterscheiden, die vierte startet mit Jänner 2011.

- Programmphase 1 (2003 bis 2005): Bereitstellung von Förderprogrammen für die BMVIT-Zielgruppen: FTI-Unternehmen, außeruniversitäre Forschungseinrichtungen sowie Fachhochschulen (damals gab es parallel dazu im BMVIT das Förderungsprogramm FHplus). Begleitet wurden diese Förderungsaktivitäten von Informationsbereitstellung, Studien und die FEMtech Expertinnendatenbank.

- Programmphase 2 (2006 bis Mitte 2008): Konzentration auf FEMtech Karriere, Einbettung in internationale Programmdiskurse (FEMtech Konferenz anlässlich der EU-Präsidentschaft Österreichs, European Platform of Women Scientists etc.) und Einbindung in die FFG-Förderungsabwicklung.

- Programmphase 3 (Mitte 2008 bis 2010): Ressourcenmäßiger Ausbau des Programms. Zusätzliche Durchführung der Förderlinien FEMtech Karrierewege und FEMtech FTI-Projekte und damit Ausweitung der Zielgruppen und Förderungsangebote. Bündelung und Anreicherung der Programmlinien „Aktivitäten“ und „Wissen“ durch umfassende Maßnahmen und Einzelaktionen.

Mit 2011 startet eine neue FEMtech Programmphase, deren Inhalte gegen Ende der Evaluierung noch nicht festgelegt waren.

In den letzten sieben Jahren ist es gelungen, die finanziellen Mittel für FEMtech kontinuierlich auszuweiten. Insgesamt wurden von 2004 (erste Förderungen) bis Oktober 2010 5.376.809 $€$ als Fördermittel eingesetzt. Zusätzlich wurden für Studien, Öffentlichkeitsarbeit sowie alle Aktivitäten und insbesondere die internationale Tagung im Mai 2006 856.144 € im Zeitraum 2003 bis Oktober 2010 eingesetzt. ${ }^{20}$ In Relation bleibt es im Portfolio der Forschungsförderung noch immer ein „kleines Programm“ (wie alle Programme im Bereich Humanressourcen).

\subsection{Organisationsstruktur}

FEMtech ist es geglückt, die Signale und das Zeitfenster der Entscheidungsmacht des Rates für Forschung und Technologieentwicklung (RFTE) zu nut-

${ }^{20}$ Aussagen zur Effizienz des Einsatzes der Mittel konnten aufgrund der pauschalen Kostenstrukturen, die dem Evaluationsteam zur Verfügung gestellt wurden, nicht getroffen werden. 
zen. ${ }^{21}$ Dieser stellte u. a. in Vorbereitung des nationalen Forschungs- und Innovationsplans fest, dass die beiden Ministerien BMVIT und BMWF gemeinsam ein Programm zur Frauenförderung (fFORTE) aufsetzen sollen. Zu diesem Zeitpunkt hatte der RFTE auch Entscheidungsmacht über Programmfinanzierungen und forderte zu „neuen Programmen“ auf - somit konnte von Seiten des BMVIT FEMtech entwickelt und umgesetzt werden.

Die oben dargestellten FEMtech Programmphasen sind wesentlich geprägt durch die jeweilige Organisationsstruktur von FEMtech, die in sieben Jahren dreimal wechselte, demnach eine starke organisatorische Herausforderung für das Programm darstellte.

- Programmphase 1 (2003 bis 2005): Die organisatorische Abwicklung von FEMtech erfolgte im Konsortium TIG (Technologie Impulse Gesellschaft), Joanneum Research und D\&Z Consulting unter dem Lead der TIG, jedoch im partnerschaftlichen Übereinkommen und in Ergänzung durch die ÖGUT (als Werkvertragsnehmerin des BMVIT). FEMtech ist geprägt von einer Aufbau- und Entwicklungsphase: Es gilt das Thema zu positionieren; Sensibilisierung findet überwiegend über breite Öffentlichkeitsarbeit bzw. in den Ministerien und FTI-Gremien statt. Organisatorisch funktioniert FEMtech wie eine Projektpartnerschaft und ist durch einen Werkvertrag vom BMVIT beauftragt.

- Programmphase 2 (2006 bis Mitte 2008): Die Technologie Impulse Gesellschaft wird in die FFG übergeführt. Damit wechselt die Kommunikations- und Abwicklungsstruktur des FEMtech Teams hin zu einem starken Lead der FFG unter Dienstleistungsbeteiligung der anderen PartnerInnen. In der Anfangsphase der FFG (als Merger von vier Vorläuferorganisationen) war diese intensiv um die Vereinheitlichung der internen Prozesse und inhaltliche Eigenständigkeit bemüht. Das Programm FEMtech mit seinem Fokus auf Awareness- und Begleitmaßnahmen war darin schwer zu integrieren, es fehlte z. T. an Flexibilität für kurzfristige Aktivitäten. Ab 2007 wurden von Seiten der FFG Ausführungsverträge mit dem BMVIT bezüglich des Programmes getätigt, d. h. es gab eine Rahmensetzung für das Programm, das BMVIT konnte keine Detailinhalte mehr bestimmen.

- Programmphase 3 (Mitte 2008 bis2010): Mitte 2008 erfolgt die vertragliche Aufteilung der Leistungserbringung von FEMtech in zwei Teile: Die FFG übernimmt ausschließlich die Förderungsabwicklung, Joanneum Research, ÖGUT, D\&Z Consulting sowie der neue Partner Bohmann-Verlag bilden das Konsortium für das FEMtech Kompetenzzentrum. Der Begriff Kompetenzzentrum war ein deutliches Signal in Richtung eines gemeinsamen Aufbaus und Transfers von Know-how zu den Themen Frauenförderung und Chancengleichheit. Tatsächlich konnten viele Leistungen neu gebündelt werden. Durch die Einrichtung des Kompetenzzentrums erhöhte sich jedoch die Komplexität der Abstimmungs- und Kommunikationsprozes-

${ }^{21}$ Diese Aussage beruht auf dem FEMtech Workshop mit BMVIT, FFG, ÖGUT und JR zur „Programmhistorie“im Rahmen der Evaluation. 
se: Da es schwer war, gemeinsam Ziele und Strategien zu finden sowie abgestimmt und kohärent zu handeln. ${ }^{22}$

Die internationalen Fallbeispiele zeigen, dass Zentren zur Stärkung von Chancengleichheit in FTI unterschiedlich organisatorisch verankert sein können. Zentral ist jedoch eine gewisse „Bewegungsfreiheit“, d. h. dass Inhalte eigenständig entwickelt und umgesetzt werden können. Während das ABI eine private Organisation ist, die durch Spenden finanziert wird, ist die MPDF eine Stabsstelle innerhalb des CNRS. Das UKRC ist mit FEMtech aufgrund der Finanzierung durch das „Department for Business, Innovation \& Skills“23 am ehesten vergleichbar. Es hat jedoch keinen Programmcharakter, sondern ist eine dauerhafte und unabhängige Einrichtung, die u. a. auch eigene politische Strategien erarbeitet. Geführt werden die Einrichtungen der drei Fallbeispiele von Direktorinnen und Direktoren. Sie werden von verschiedenen Gremien in ihrer Arbeit unterstützt. Das UKRC wird von einem Governing Body und einem Industry Board beraten. Unterstützung erhält das ABI von einem Board of Trustees und einem Board of Advisors. Alle drei Einrichtungen versuchen regional präsent zu sein. Das UKRC hat fünf regionale Anlaufstellen. Dies ist aufgrund der Größe des Vereinigten Königreichs naheliegend. Die MPDF des CNRS hat keine dezentralen Stellen, sondern wird regional durch seine Delegierten und ihr Team repräsentiert. Das ABI ist stark auf virtuelle Vernetzung ausgerichtet und hat daher keine regionalen Vertretungen.

Insgesamt wurde die Organisations- und Umsetzungsstruktur - die durch mehrere Partnerinnen und viele Teammitglieder (2010 an die 10 Personen) repräsentiert wurde - darauf ausgelegt, Informationen aufzubereiten, Veranstaltungen durchzuführen und die Öffentlichkeitsarbeit zu organisieren. ${ }^{24}$

Die MitarbeiterInnen werden von den Zielgruppen als besonders engagiert beschrieben und haben durch ihr persönliches Engagement und ihr Know-how die Awareness- und Sensibilisierungsphase von FEMtech erfolgreich aufgesetzt.

Der finanzielle Mitteleinsatz für die Abwicklungs- und Begleitungsstruktur von FEMtech ist aufgrund der pauschalen Angaben des BMVIT zu den Gesamtkosten des Programms nicht exakt zu ermitteln. Zudem waren zu unterschiedlichen Zeitpunkten verschiedenartige Leistungen in die organisatorische Begleitung inkludiert.

\subsection{Embedding}

Durch das Drängen des Rates für Forschung und Technologieentwicklung wurden das BMVIT und das BMWF aufgefordert, abgestimmt und koordiniert Programme zur Förderung von Frauen in Wissenschaft, Forschung und Technologie aufzusetzen. fFORTE - Frauen in Forschung und Technologie - wurde als Dach der Frauenförderung der beiden Ministerien gegründet, später kamen

\footnotetext{
${ }^{22}$ Quelle: 7 von 10 der Befragten lt. Self-Assessment der ProgrammumsetzerInnen

23 http://www.bis.gov.uk/

${ }_{24}$ Quellen: Programmgespräche ProgrammumsetzerInnen, Interviews ehemalige Programmleiterinnen
} 
noch w-fFORTE (BMWFJ) und fFORTE Schule (BMUKK) hinzu. Die Intention des RFTE, ministeriumsübergreifend unterstützende Maßnahmen zur Frauenförderung anzubieten, war lobenswert. Jedoch konnte sich die Dachinitiative nicht den strukturellen Hindernissen, welche sich aus einer Aufteilung der thematischen Zuständigkeit auf mehrere Ministerien ergeben, entziehen. So wurde im Rahmen von fFORTE zwar informiert, es konnte jedoch nicht erreicht werden, dass Maßnahmen abgestimmt oder gemeinsam umgesetzt werden.

FEMtech versuchte in jeder Programmphase, internationale Kontakte zu Einrichtungen im Bereich Frauenförderung in Wissenschaft, Forschung und Technologie zu knüpfen. In Programmphase 1 waren insbesondere Zentren in Deutschland Vorbilder (FEMtec Berlin, das Kompetenzzentrum Frauen in Informationsgesellschaft und Technologie in Bielefeld sowie das Kompetenzzentrum Frauen in Wissenschaft und Forschung CEWS in Bonn). Diese Kontakte waren sporadisch und wurden nicht weiter ausgebaut. In der Programmphase 2 fand eine internationale Konferenz im Rahmen der EU-Präsidentschaft Österreichs in Wien statt und erreichte damit auf europäischer Ebene Aufmerksamkeit. FEMtech richtete die Konferenz aus und konnte sich gut präsentieren. Zahlreiche Beteiligung aus ganz Europa gab dem Thema Frauenförderung in Forschung und Technologie Aufschwung. In der Programmphase 3 wurde vom Kompetenzzentrum die Einbindung in die (wissenschaftliche) Chancengleichheits- und Gender-FTI-Community in Deutschland und der Schweiz gesucht. Ein systematischer kooperativer Austausch, der über Fachtagungen hinausgeht, wurde nicht aufgebaut.

Embedding, die Einbindung von FEMtech in die FTI-politische Community, fand nicht essenziell statt. Dies hängt mit der strukturellen Trennung als „Frauenförderungsprogramm“ von anderen FTI-politischen Maßnahmen zusammen. Diese strukturellen Schwierigkeiten führten zu einer Konzentration auf die Umsetzung der FEMtech Fördermaßnahmen und die damit verbundene Öffentlichkeitsarbeit. Im Zusammenwirken des Kompetenzzentrums wurde fachliche Expertise aufgebaut.

Im Vergleich zu den drei internationalen Fallbeispielen ist der Vernetzungsgrad des FEMtech Kompetenzzentrums geringer: Alle drei Einrichtungen sind intensiv vernetzt und setzen auf externe Expertise. Während dies beim ABI auch durch die Abhängigkeit von den Sponsoren bedingt ist, dient die Vernetzung beim UKRC mit den Universitäten vor allem der Sicherstellung einer wissenschaftlichen Grundlage für die Programme und Aktivitäten. Das ABI hat neben den Sponsoren auch ein differenziertes System von UnterstützerInnen, insbesondere Partner-Organisationen, BotschafterInnen, UnterstützerInnen im Internet, die Werbung für ABI machen und Freiwillige, die Mailing-Listen moderieren, Workshops organisieren oder in Gremien aktiv sind. Auch die MPDF des CNRS ist regional, national und auf europäischer Ebene vernetzt, um seiner Rolle als Observatorium gerecht zu werden. Das UKRC und das ABI fördern Vernetzung nicht nur auf der direkten Ebene mit Partnerorganisationen oder wissenschaftlichen Einrichtungen, sondern initiieren und begleiten auch Netzwerke von Fachgesellschaften (UKRC) und Frauen der verschiedenen Fachrichtungen in Forschung und Technologie (ABI). Besonders hervorzuheben ist die vom UKRC initiierte und begleitete Vernetzung von 
Fachgesellschaften im MINT-Bereich (SPIDER). ${ }^{25}$ Es bietet den Mitgliedern neben Netzwerk-Veranstaltungen z. B. eine Datenbank zu Case-Studies und Best-Practice-Ideen, Online-Leitfäden, Beratung und Training zu Gleichstellung sowie regelmäßige Informationen zu Chancengleichheit.

\subsection{Conclusio}

FEMtech hat in seiner siebenjährigen Geschichte bereits drei unterschiedliche Phasen der Programmschwerpunkte und Organisationsstruktur erlebt, die vierte Phase beginnt mit 2011. Dies sind viele Umbrüche, die auch durch Zeiten des Stillstands, inhaltlichen Neukonzeptionen und Neuorientierungen geprägt waren. Jahres- und Halbjahresverträge zur Programmabwicklung und -betreuung trugen wenig zur Langfristigkeit bei. Dennoch wurden diese Veränderungen von außen nicht tiefgreifend wahrgenommen. Dies liegt vor allem an der weitgehenden Kontinuität der programmumsetzenden Personen und Organisationen.

FEMtech als Programm zur Frauenförderung und Chancengleichheit fand zwar Aufmerksamkeit, jedoch zu wenig Anerkennung in der FTI-politischen Community, die sich eher an Fördervolumina und „klassischen“ Programmförderungen orientiert. Das Kompetenzzentrum war eine Art „Dienstleistungszentrum" für das BMVIT. So gingen weder von den FTI-politischen AkteurInnen noch von den Programmverantwortlichen intensive Signale zur Kooperation und Zusammenarbeit aus. Dies verursachte einen Mangel an Kommunikation mit relevanten AkteurInnen und den Zielgruppen, wenig Kooperations- und eine verstärkte Binnenorientierung. Intern dominiert ein FTIpolitischer und wissenschaftlicher Zugang zum Thema, der von der Zielgruppe, insbesondere privatwirtschaftlichen Unternehmen, nicht unbedingt verstanden wird. Im Außenauftritt bleiben die Programme der einzelnen Ministerien getrennt, die Möglichkeiten der Kooperation sind durch die verteilte Verantwortlichkeit eingeschränkt.

Um in Zukunft vermehrt die Zielgruppen mit ihren Bedarfen zu adressieren, bedarf es einer strukturellen Öffnung des Programms. D. h. es braucht Signale und aktive Einladungen zur Zusammenarbeit auf unterschiedlichen Ebenen. Umgekehrt benötigt FEMtech auch Kooperationsangebote FTI-politischer AkteurInnen, die sich zunehmend mit dem Thema Chancengleichheit beschäftigen müssen. Für ein Mainstreaming und eine breite Verankerung der Thematik ist u. a. ein kontinuierlicher Erfahrungsaustausch und Wissenstransfer zwischen unterschiedlichen Programmen notwendig. Maßnahmen, wie Trainings zum Thema Chancengleichheit und Gender Mainstreaming, können hier einen wichtigen Beitrag leisten, um die notwendigen Voraussetzungen für die Bereitschaft zu einem derartigen Diskurs zu schaffen (wie bspw. bereits innerhalb der FFG durchgeführt).

Vernetzung der Reflexion österreichischer Erfahrungen im internationalen Vergleich bzw. mit anderen europäischen Einrichtungen kann ebenfalls einen wichtigen Beitrag zur breiten Verankerung der Thematik leisten.

\footnotetext{
${ }^{25} \mathrm{http} / / / \mathrm{www}$. theukrc.org/for-organisations/for-professional-bodies/join-spider
} 


\section{Schlussfolgerungen \& Empfehlungen}

\subsection{Schlussfolgerungen}

FEMtech hat zur Aufgabe, den langfristigen Prozess des Mainstreamings von Chancengleichheit in der FTI-Förderung - speziell diejenigen des BMVIT - zu gestalten und mit sinnvollen Maßnahmen zu unterstützen. FEMtech ist dabei mehr als ein FTI-Programm, da es langfristig gleichstellungspolitische Fragestellungen in alle Planungs- und Entscheidungsprozesse der FTIFörderungspolitik integrieren will. Damit wird Gleichstellungspolitik aus der Ressortzugewiesenheit von Forschungsförderung gelöst und zum Auftrag an die FTI-Politik und an alle AkteurInnen in diesen Prozessen.

Erfolgreiches Mainstreaming von Chancengleichheit kann nur dann gelingen, wenn es als Bestandteil von Veränderungsprozessen in der FTI-Politik und Forschungsförderung umgesetzt und selbst als Organisations- und Veränderungsprozess (forschungsförderungsintern, speziell BMVIT und FFG) gestaltet wird.

Ein Mainstreamingprozess beeinflusst einen „kulturell-mentalen Wandel“ und benötigt daher einen langen Zeitraum. Eine kurzfristige Erhöhung des Frauenanteils in Forschung und Technologie kann somit als politische Zielvorstellung, jedoch nicht als kurzfristige handlungswirksame Orientierung erachtet werden, da hierzu im Vorfeld umfassende Bewusstseins- und Verhaltensänderungen stattfinden müssen.

Abbildung 47: Mainstreamingprozess - Einordnung Programm FEMtech

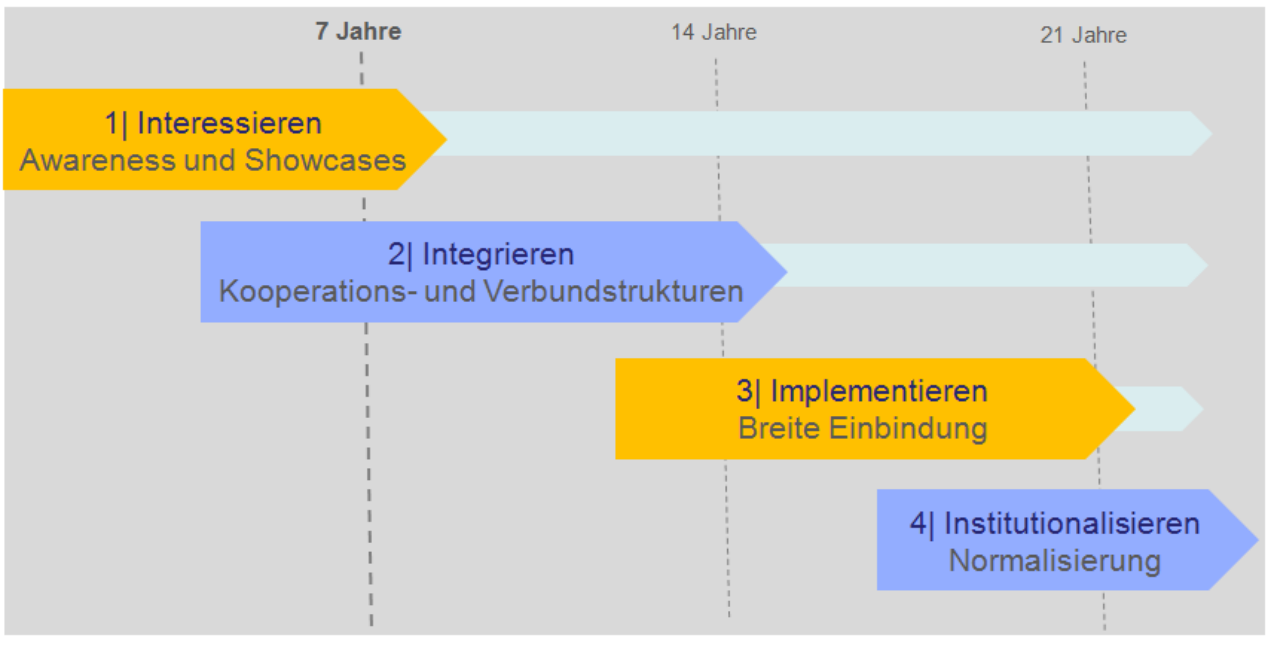

Quellenangabe: ARGE „3C“-Analyse

Ein Mainstreamingprozess erfolgt in vier Stufen und wurde hier in 7-JahresPhasen eingeteilt: Die 7 Jahre basieren einerseits auf der Erfahrung des Evaluationsteams aus ähnlichen Prozessen, andererseits auf Einschätzungen der im Evaluationsprojekt interviewten FTI-AkteurInnen: Auf die Frage: „Angenommen FEMtech wäre ein Marathon: Bei welchem Kilometerstand ist das Programm jetzt?“ lautete die häufigste Antwort „Kilometer 15, d. h. ein Drittel des Weges ist geschafft". FEMtech lässt sich wie folgt in den skizzierten Mainstreamingprozess einordnen: 
1. Interessieren | Sensibilisierung: FEMtech ist es gelungen, durch aktive Awarenessmaßnahmen sowie durch Schaffung von Showcases für die Themen Chancengleichheit und Frauenförderung in der FTIForschungsförderung zu sensibilisieren. Wie bereits festgestellt wurde: Awareness ist erreicht, die erste Phase wurde erfolgreich bewältigt.

2. Integrieren | Kooperations- und Verbundstrukturen: FEMtech hat es verabsäumt, kontinuierlich am Aufbau von Kooperations- und Verbundstrukturen zu arbeiten und hier entsprechende Angebote auf unterschiedlichen Beteiligungsniveaus anzubieten. ${ }^{26}$ Einzelne Aktivitäten dazu wurden gesetzt, jedoch nicht systematisch entwickelt und weitergeführt. Daher fehlt die „Verwurzelung“ des Programms auf Ebene von MultiplikatorInnen und Netzwerkknoten, die entscheidend für eine substanzielle Verbreiterung im Sinne des Mainstreamings sind.

3. Implementieren | Breite Einbindung: FEMtech möchte nun - das neue wirkungsorientierte Bundeshaushaltsgesetz als Hebel nutzend - Genderkriterien in die Basisprogramme der FFG sowie in Zukunft in weitere FFGProgramme implementieren.

Durch das Überspringen der Phase 2 ist die Implementierungsphase schwach aufgestellt: Zu wenig Erfahrung der Fördereinreichenden, zu wenig Rückhalt von weiteren förderungspolitischen AkteurInnen lassen befürchten, dass „Genderkriterien“ bei Fördereinreichungen mehr Farce sind, als dass sie Entscheidungsrelevanz besäßen. Ein Interviewpartner formuliert dies wie folgt: „Das passiert unter dem Motto: Die wissen, dass ich weiß, dass das nicht das Wichtigste ist, und umgekehrt." (Zitat FTI-pA1) 
Einen zweiten Gesamtblick auf das Programm ermöglicht nachstehende Grafik: Die FEMtech Österreichkarte.

Abbildung 48: FEMtech Österreichkarte

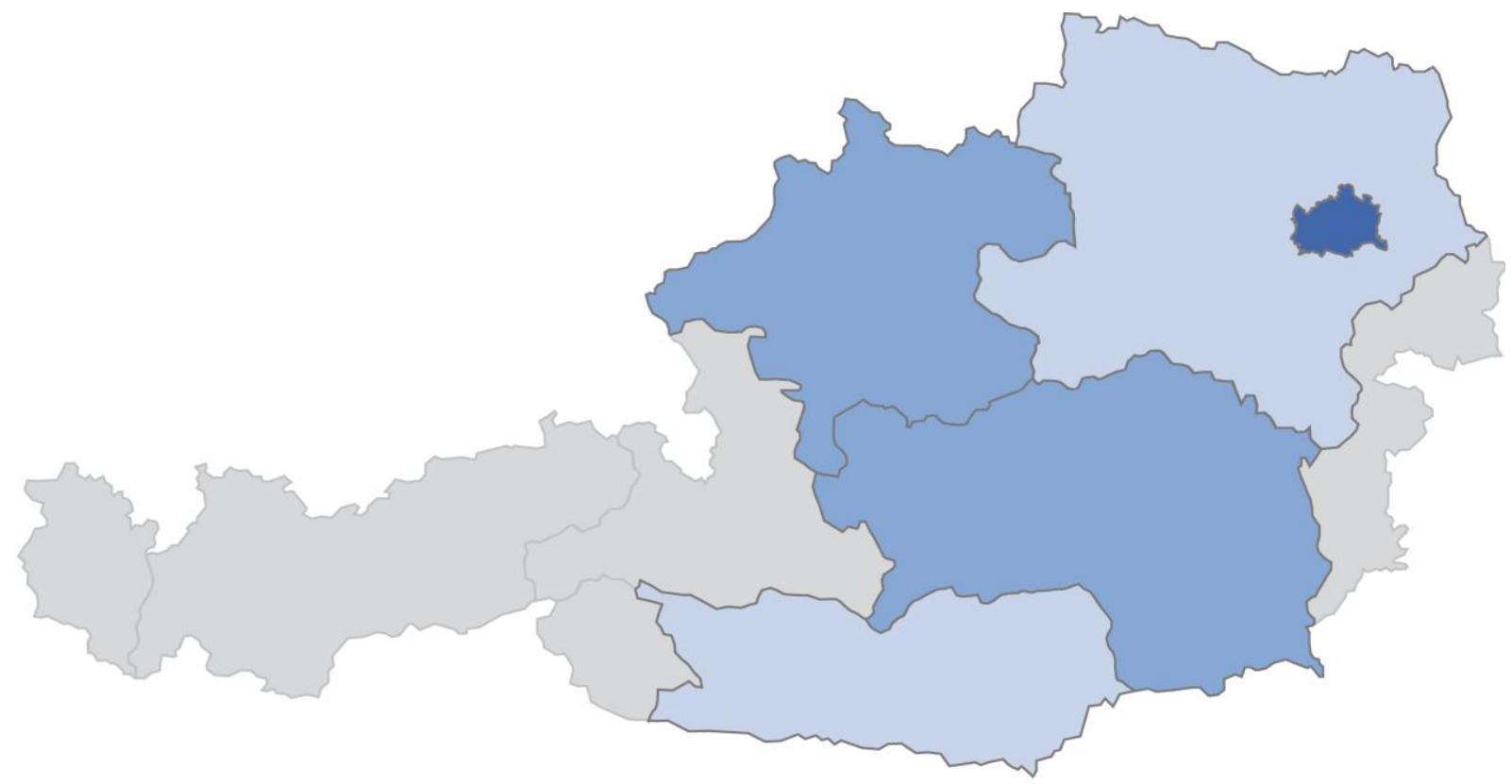

Quellenangabe: ARGE „3C“-Analyse

Die FEMtech Österreichkarte basiert auf der Bekanntgabe des Bundeslandes bei Fördereinreichungen und der in der Onlinebefragung ermittelten NutzerInnenstruktur in den Bundesländern. Sie überrascht nicht, denn eine Österreichkarte über alle FTI-Förderungen würde ähnliche Ergebnisse zeigen. Dennoch: In der Analyse zeigt sich, dass FEMtech die bundesländerspezifischen Unterschiede in der FTI-Förderung noch verstärkt. D. h. in besonders aktiven Bundesländern ist FEMtech noch reger, in Bundesländern, die in der Forschungsförderung wenig präsent sind, ist FEMtech kaum vertreten. FEMtech ist mit Projekten und Aktivitäten stark auf die Bundeshauptstadt konzentriert (zu ca. 50 \% bis $60 \%$ ). Die Steiermark und Oberösterreich sind bei Fördereinreichungen gut vertreten, Oberösterreich hier sogar überproportional hoch. Dafür gibt es in der Relation überraschend wenige FEMtech NutzerInnen aus Oberösterreich in der Programmlinie FEMtech Aktivitäten. Die Steiermark ist - auch über die TU Graz - hier gut eingebunden. Kärnten zeigt sich - im Österreichvergleich - bei FEMtech besser vertreten. Wie bereits erwähnt, hängt dies vor allem mit der WITEC-Österreichvertretung in Villach zusammen. Noch wenige Projekte und FEMtech NutzerInnen stammen aus Salzburg und Tirol; Vorarlberg und Burgenland sind kaum existent.

Die FEMtech Österreichkarte spiegelt zugleich auch wider, dass im Mainstreamingprozess für Chancengleichheit die zweite Phase zur Bildung von Kooperationen und Verbundstrukturen bisher nicht ausreichend vorbereitet wurde. Kooperationen mit MultiplikatorInnen, Netzwerkknoten und FTIEinrichtungen sind vor allem auf Bundesländerebene zu bilden und würden zusätzlich die regionale Verankerung des Programms FEMtech stärken. 
Nach diesen einleitenden Ausführungen zur Einordnung von FEMtech in den Mainstreamingprozess und seiner österreichweiten Präsenz erfolgen im Weiteren konkrete Schlussfolgerungen entlang den Fragestellungen:

- Was ist im Programm gut gelungen?

- Was ist problematisch im Programm?

- Was ist für die zukünftige Weiterführung des Programms als kritisch zu erachten?

\subsubsection{Gut gelungen im Programm}

FEMtech ist es sehr gut gelungen, „windows of opportunites“ zu nutzen, um Frauenförderung im FTI-politischen Bereich aufzugreifen, anzusprechen und konkrete Maßnahmen umzusetzen. Dies betrifft sowohl das Gesamtprogramm als auch einzelne Aktivitäten, die schnell und zeitgerecht erfolgten. Nicht alle Aktivitäten zeigten sich dabei als erfolgreich, die Themenpalette wurde dadurch jedoch angereichert und Erfahrungen gesammelt.

Die Konzeption, das Programm FEMtech als Kombination aus Awarenessmit Fördermaßnahmen umzusetzen, war klug und hat funktioniert. FEMtech ist das erste technologiepolitische Programm in Österreich, das zu seinem Förderprogrammen nicht lediglich „Begleitmaßnahmen“ anbietet, sondern einen breiten Sensibilisierungs- und Awarenessprozess aufsetzt. Es war von Anfang an klar, dass Frauenförderung und Chancengleichheit hier andere Prozesse und Maßnahmen benötigt als bisherige thematische und strukturbezogene Programme.

Phase 1 des Mainstreamings von Chancengleichheit: Schaffung von Awareness und Showcases wurde erfolgreich durchgeführt. Konkret bedeutet dies:

- Awareness wurde erreicht, das Thema ist bei den Zielgruppen angekommen. Der Bekanntheitsgrad von FEMtech ist in der FTI-politischen Community hoch.

- Die Vernetzung der „Interessierten“ bzw. bereits „Sensibilisierten“ findet erfolgreich statt, der Kreis erweitert sich ständig.

- FTI-politische AkteurInnen bestätigen, dass nun anders über Chancengleichheit in der Forschungsförderung gesprochen wird und dass insbesondere bei der Besetzung von Jurys, Boards etc. hier mehr Aufmerksamkeit herrscht.

- Es ist gelungen, durch die FEMtech Förderungen Showcases zu schaffen. Die erfolgreichsten und besten können in Zukunft als Beispiele für andere Unternehmen und Forschungseinrichtungen aufbereitet werden.

- Die FEMtech NutzerInnen sind in der Regel zufrieden, d. h. die Programmlinien Förderungen, Aktivitäten und Wissen werden gut bewertet.

- Programmwirkungen entstanden insbesondere im Bereich der Sensibilisierung, weniger im organisatorisch-strukturellen Bereich und kaum im Hinblick auf veränderte Zahlen bzw. Frauenanteile. 


\subsubsection{Problematisch für das Programm}

Frauenförderung und Chancengleichheit sind neue Themen für die FTI-Politik und sind in ihrer emotionalen Dimension anders zu bewerten als andere Schwerpunkte (wie Energie, Mobilität, Nachhaltigkeit etc.), da sie grundlegende Werte und gesellschaftliche Rollenbilder berühren. NutzerInnen von FEMtech sind damit überwiegend themenaffine Personen und Organisationen. Projekte im Bereich FEMtech Förderungen erreichen vor allem bereits sensibilisierte Personen und Organisationen. Problematisch wird es dann, wenn Personen, die sich mit Frauenförderung und Chancengleichheit befassen, unter sich bleiben und somit die notwendige Breite im Mainstreamingprozess nicht erreicht wird.

FEMtech ist die Schaffung von Awareness geglückt. Dennoch, Frauenförderung und Chancengleichheit sind noch nicht im Sinne eines Mainstreamings „im FTI-politischen Kern angekommen“. Viele der interviewten FTIpolitischen AkteurInnen meinen, dass die nächste Phase der Themenverankerung in der FTI-Politik viel schwieriger wird, als das Schaffen von Awareness war.

Die experimentelle Ausrichtung hat zu einer mangelnden Kontinuität in Zielen und Strategien sowie in der Ableitung von Maßnahmen geführt. Zum Teil wurden einzelne (unterkritische) Maßnahmen gesetzt, die jedoch nicht weiter betreut wurden und somit keine langfristige Wirkung erzielen konnten. Diese Vorgangsweise ist eventuell in der Phase 1 des Mainstreamings noch hilfreich, da Aufmerksamkeit in unterschiedlichen Feldern breit erreicht wird. Für die Fortführung des Programms wäre dies jedoch problematisch, da zu wenig Stringenz, Kohärenz und Orientierung (auch in Hinblick auf Kooperationen) vorhanden sind.

Entsprechend der experimentellen Ausrichtung wurden auch Zielgruppen hinzugefügt, geändert und in einzelnen Zeitabschnitten unterschiedlich intensiv betreut. Die „Zielgruppenlogik“ war dabei politisch-handlungsorientiert: „Was hätten wir gerne verändert, und wen brauchen wir dazu?" Wenn zukünftig verstärkt privatwirtschaftliche Unternehmen erreicht werden sollen, so ist es notwendig, kontinuierlich den Nutzen aus der Sicht der Unternehmen zu kommunizieren und entsprechende Materialien anzubieten, die ihren spezifischen Anforderungen gerecht werden. Kommunikation und Angebote bedürfen der Sprache der jeweiligen Zielgruppe.

Die wechselnden Zuständigkeiten, Aufgaben und Funktionen in der Programmabwicklung und -begleitung wurden von den Zielgruppen und den FTI-politischen AkteurInnen z. T. als verwirrend empfunden. 2011 startete die insgesamt vierte Phase in der FEMtech Programmbetreuung innerhalb von gut sieben Jahren. Dies dürfte mit ein Grund sein, warum wenig Kontinuität in Zielen und Strategien feststellbar ist und der Ressourceneinsatz im FEMtech Programm nicht immer optimal verlief. 


\subsubsection{Kritisch für das Programm}

Kritisch für das Programm werden vom Evaluationsteam diejenigen Punkte erachtet, die für eine erfolgreiche Weiterführung des Programms hinderlich sind. Sie betreffen drei Bereiche:

Die Marke FEMtech ist als Wort-Bild-Marke bei der Gruppe der Interessierten, den Zielgruppen und den FTI-politischen AkteurInnen präsent und gut verankert. Dennoch deuten die Evaluationsergebnisse darauf hin, dass die Bezeichnung „FEMtech“ in Zukunft auch hinderlich sein kann. Es zeigte sich, dass „Chancengleichheit“ der Begriff ist, mit dem insbesondere FTIUnternehmen wesentlich besser umgehen können. FEMtech löst bei allen Befragten jedoch die Assoziation „Frauenförderung“ aus, was per se am Wortbestandteil „FEM“ liegt. Frauenförderung als Maßnahme lehnen viele FTIUnternehmen jedoch ab, weil sie im Unternehmen keine diskriminierenden Maßnahmen - auch keine positiven - setzen möchten. Chancengleichheit wird in einem viel stärkeren Ausmaß befürwortet. Wenn daher in Zukunft vermehrt mit „Chancengleichheit“ argumentiert werden sollte, kann der Name FEMtech als „kommunikative Bremse“ wirken (vgl. Kapitel 3.4.3 „Agenda-Setting in der FTI-Community“).

Die beiden Hauptzielgruppen der Forschungsförderung sind FTI-Unternehmen und außeruniversitäre Forschungseinrichtungen. Während letztere gut mit diversen Rahmenbedingungen und Vorgaben der Forschungsförderung und der FTI-Politik umgehen können, müssen FTI-Unternehmen von FEMtech gezielt angesprochen werden. Dazu bedarf es einer Konzentration der Ressourcen und Kräfte auf diese Zielgruppe und passender Angebote und Maßnahmen in ihrer „Sprache“.

Das Überspringen der Phase 2 im Mainstreamingprozess schafft für die Zukunft ernsthafte Probleme: Das Programm FEMtech trägt alleine die Sensibilisierungs-, Informations- und Motivationsarbeit für Aktivitäten und Förderungen, die Akquisition von Unternehmen hat sich als äußerst mühevoll erwiesen. Wenn dies nicht auf eine breitere Basis gestellt wird, indem MultiplikatorInnen, FTI-Einrichtungen etc. auf Bundes- und regionaler Ebene als Kooperationspartner und Verbündete gewonnen werden, tritt das Programm bestenfalls auf der Stelle, ein substanzieller Fortschritt in Richtung Chancengleichheit wird nicht zu erwarten sein.

\subsection{Empfehlungen}

Die nachfolgend formulierten Empfehlungen richten sich an die Steuerung des Programmes und reichen darum über die Diskussion einzelner Detailverbesserungen hinaus. Abgeleitet aus den Ergebnissen sämtlicher Arbeitspakete der Evaluierung halten die EvaluatorInnen eine Konzentration der Ziele, Strategien, Aufgaben und Maßnahmen bei gleichzeitig breiterer Einbindung in die FTI-politische Multiplikatorenlandschaft für notwendig. Daraus leiten sich folgende Empfehlungen entlang der FEMtech Programmlinien $\mathrm{ab}$ : 


\section{Förderungen}

Die Kombination aus Förderungen und Awareness sind für das Programm FEMtech wichtig und sollen auch weiterhin in der Programmkonzeption Anwendung finden.

\section{FEMtech Karriere}

FEMtech Karriere soll auch in Zukunft durchgeführt werden, wobei drei Adaptionen in der Grundstruktur erfolgen sollten:

1. Die Förderlinie soll, um zukünftig mehr privatwirtschaftliche Unternehmen gewinnen zu können, bisherige Ergebnisse unterstützend aufbereiten. Es könnten beispielartig einzelne Module, welche zur Chancengleichheit beitragen, dargestellt werden, um diese entsprechend gut kommunizieren zu können (Baukastensystem).

2. Die Abwicklung soll vereinfacht werden: Die Formulierungen im Leitfaden und Antrag sollen so erfolgen, dass sie von einreichenden Zielgruppen, die nicht FFG-Stammklientel sind (z. B. GeschäftsführerInnen von KMU, PersonalistInnen etc.) verstanden werden und zu bewältigen sind. Zusätzliche statistische Erhebungen (,Datenblätter“) sollten die bisher offenkundig bestehende Einreichhürde nicht erhöhen. Es ist zu überlegen, ob mit dem zukünftigen „Baukastensystem“ externe Fachgutachten noch notwendig sind. Da der Maßnahmen-Ressourceneinsatz bereits vorweg geklärt wäre, würde eventuell auch eine FFG-interne Prüfung machbar sein. Ein solches Vorgehen würde die „time to contract“ beschleunigen, ohne die Qualität der Entscheidungen zu beeinflussen.

3. Innerhalb der Förderungsbeschreibung (auf Website, Programmdokumenten etc.) soll besser zwischen Einreichberechtigten und Zielgruppen der Maßnahmen bzw. direkten Zielgruppen (FTI-Unternehmen und -Organisationen) sowie indirekten Zielgruppen (Frauen und Männer, deren Chancengleichheit durch die Maßnahmen erhöht wird) unterschieden werden. Im Sinne der Chancengleichheit ist bei den Zielgruppen darauf zu achten, dass die Darstellung nicht zu sehr zu einer Assoziation mit Frauenförderung führt.

\section{FEMtech Karrierewege}

FEMtech Karrierewege wurde als Pilotprojekt umgesetzt. Die 6 geförderten Projekte weisen prinzipiell eine Kooperation zwischen Wissenschaft und Wirtschaft auf. Die langfristig erzielte Wirkung, im Sinne einer Erhöhung des Frauenanteils in der Industrie, ist jedoch nicht erkennbar (vgl. Abbildung 37), allerdings ist die mögliche langfristige Wirkung nicht direkt messbar. Diese große Bandbreite der Maßnahmen, welche auf unterschiedlichsten Ausbildungsniveaus ansetzen, führt zu einer großen Herausforderung in der strategischen Ausrichtung der Begleitmaßnahmen, um alle Bedürfnisse der im Projekt unterstützten Frauen adressieren zu können. Die Weiterführung der Programmlinie in der derzeitigen Ausführung wird daher als weniger effektiv angesehen. Um die Strategien der Projekte besser zu planen, ist eine Fokussierung auf eine homogenere Zielgruppe anzustreben. Um einen Mehrwert für Unternehmen, Universitäten und Studentinnen zu generieren, ist eine Förderung ab Abschluss des Bakkalaureats anzustreben. Dies garantiert eine fundierte Basisausbildung, welche es den Studentinnen ermöglicht, sich einfacher 
in die Teams und Arbeitsgruppen von FTI-Unternehmen zu integrieren und vollwertig aufgenommen zu werden (sei es in Zusammenhang mit Praktika oder auch Diplomarbeiten bzw. Masterarbeiten). Eine Mindestdauer von 3-4 Monaten ist hier ebenfalls förderlich, da vor allem durch Kurzpraktika von einigen Wochen eine nachhaltige Integration in die Unternehmen nur in Ausnahmefällen möglich wird. Der Abschluss des Masterstudiums stellt einen entscheidenden Meilenstein in der weiteren Karriereentwicklung und ausrichtung dar.

Begleitmaßnahmen sollten in enger Abstimmung mit den Unternehmen geschehen, um auch hier eine verbesserte Unternehmenskultur in Bezug auf Chancengleichheit zu erreichen.

\section{FEMtech FTI-Projekte}

Die Förderlinie FEMtech FTI-Projekte wird vor allem von bereits sensibilisierten Personen und Organisationen aus der Wissenschaft (universitär und außeruniversitär) genutzt. Zukünftig sollte auch hier eine Verstärkung der Teilnahme durch FTI-Unternehmen angestrebt werden. Dies passiert nicht automatisch durch die bereits durchgeführten Projekte aus dem Wissenschaftsbereich, sondern erfordert aktive Transferarbeit in Richtung Unternehmen. Hier gilt es, unterschiedliche Zugangsstufen zu entwickeln und Informationen passend aufzubereiten. Dazu braucht es derzeit noch Showcases, damit hier ein gut geplanter und aufbereiteter Wissenstransfer für andere Forschungsförderungsprogramme (Basisprogramme) erfolgen kann. Mittelfristig und nach erfolgtem Transfer soll "Gender" als Forschungsschwerpunkt in allen Forschungsförderungsprogrammen Eingang finden. Bis dahin wird die weitere Ausschreibung von FEMtech FTI-Projekten - mit Konzentration auf die Zielgruppe FTI-Unternehmen und mit entsprechenden Begleit- und Transfermaßnahmen - empfohlen.

Insgesamt sollen die Ergebnisse der Förderlinien in der Kommunikation besser auf die Zielgruppen abgestimmt werden, die Fördernehmenden ein gutes Begleitangebot vorfinden (Vernetzung von Fördernehmenden, Gendertrainings als Vorbereitung für FTI-Projekte etc.) und die Showcases für die Sensibilisierung der Forschungsförderungscommunity genutzt werden.

\section{Aktivitäten}

Um die öffentliche Diskussion über Chancengleichheit und Frauen in FTI weiter zu stärken und aktuell zu halten, ist es notwendig, auch in Zukunft mit aufmerksamkeitsstarken und sensibilisierenden Maßnahmen (etwa der Präsentation erfolgreicher Frauen in FTI im Rahmen von „Expertin des Monats“) in Kombination mit Presse- und Öffentlichkeitsarbeit eine kontinuierliche Präsenz in der öffentlichen Wahrnehmung zu schaffen. Ein permanent aufrechterhaltener Themendruck trägt zudem dazu bei, die FEMtech Ziele auf FTIpolitischer Ebene im Bewusstsein zu halten und wirkt darüber hinaus unterstützend auf Bildung und Ausbau von Kooperationsstrukturen.

Die Netzwerktreffen sind eine geeignete Maßnahme, um FEMtech zukünftig stärker in den Regionen zu verankern. Über Kooperationen mit regionalen FTI-/Wirtschaftsorganisationen, Bildungseinrichtungen und Interessenvertretungen lassen sich lokale FTI-Communities zugleich breit und zielgruppenspe- 
zifisch ansprechen. Zudem kann eine Ausweitung der Netzwerktreffen in die Bundesländer der häufig geäußerten Wahrnehmung von FEMtech ErstnutzerInnen entgegenwirken, wonach sich dort insbesondere „eine enge Community“ träfe, die die FEMtech Ziele bereits seit Jahren kennt.

Die Konzentration auf eine Auszeichnung (nicht mehrere) und die damit verbundene Preisverleihungsveranstaltung bietet ein hohes Potenzial für eine öffentlichkeitswirksame und mediale Schaffung von Aufmerksamkeit und für die Mobilisierung schwer erreichbarer Zielgruppen wie privatwirtschaftliche Unternehmen. Im Sinne der Fokussierung sollten die notwendigen Ressourcen für Akquise und Begleitkommunikation jedoch auf einen Preis/eine Auszeichnung gebündelt werden - wobei deutlich wurde, das sich sowohl einreichende Organisationen als auch Medien viel eher über das Thema Chancengleichheit als über Gender ansprechen lassen.

\section{Wissen}

Um einen größeren AdressatInnenkreis zu erreichen und eine entscheidende Rolle in der Wissensvermittlung, aber auch in der Prägung von Themen und Diskursen zu spielen, sollte FEMtech sein Informationsangebot entschlacken, inhaltlich stärken und vor allem zielgruppenspezifisch aufbereiten und zugänglich machen.

Hierzu bedarf es nicht nur eines statistischen Monitorings, sondern auch einer kontinuierlichen wissenschaftsgestützten Beobachtung der nationalen, europäischen und internationalen Entwicklungen. In diesem Zusammenhang wird vorgeschlagen, das Zahlenmaterial stärker aufzubereiten und einer größeren, evtl. fachspezifischen Medienöffentlichkeit zu präsentieren.

Die Informationen sollten zudem stärker themen- und zielgruppenspezifisch aufgearbeitet werden. Um insbesondere die FTI-Unternehmen besser zu erreichen, sollte bei den eigenen Publikationen ein Schwerpunkt auf praxisorientierten, zielgruppenspezifischen Handlungsanleitungen, Leitfäden und Informationsbroschüren liegen.

\subsection{Priorisierte Empfehlungen}

Um die möglichen Entwicklungsrichtungen von FEMtech zu schärfen, priorisiert das Evaluationsteam abschließend seine Empfehlungen auf der Basis der bisherigen Analysen und Empfehlungsbereiche. Die wichtigsten drei Handlungsschritte für eine effektive Durchführung von FEMtech ab 2011 werden für die Programmverantwortlichen prägnant und stichwortartig wie folgt zusammengefasst: 


\section{Schärfung und Konsistenz der gleichstellungspolitischen Ansätze}

Sowohl in der Kommunikation als auch in der Planung und Umsetzung von Maßnahmen ist es hilfreich, auf die gewählten gleichstellungspolitischen Ansätze klar Bezug nehmen zu können. Die Förderung der Chancengleichheit soll Frauen und Männern vergleichbare Erfolgschancen bieten. Entsprechend können Maßnahmen der Chancengleichheit beide Gruppen betreffen bzw. diese einbeziehen.

Der gewählte Ansatz muss stringent nach außen dargestellt werden, damit die Erwartungen der Zielgruppen daran anschließen können. Dies ist insbesondere in der Kommunikation mit Unternehmen wichtig, für die Überlegungen des wirtschaftlichen Nutzens einer erhöhten Chancengleichheit eine wichtige Rolle spielen.

\section{Konzentration und Fokussierung}

Chancengleichheit setzt strukturelle Veränderungen in Unternehmen und Forschungseinrichtungen voraus. Nicht alle Maßnahmen im Rahmen von FEMtech garantieren eine strukturelle Verankerung der im Rahmen der Förderung erzielten Ergebnisse.

Die beiden Hauptzielgruppen können bei der Verankerung der Ergebnisse durch Begleitmaßnahmen unterstützt werden: Die beiden Förderlinien „Karriere“ und „FTI-Projekte“ benötigen ebenso wie die Linie „Karrierewege“ mehr Service, Beratungs- und Vernetzungsleistungen, um einen Transfer zu erreichen.

\section{Regionalisierung und Kooperation}

Netzwerktreffen und die Bewerbung von Maßnahmen benötigen über die bereits interessierten Personen hinausgehend unterschiedliche Angebote für Kooperationen und Verbundstrukturen, speziell mit MultiplikatorInnen und AkteurInnen der Wirtschafts- und Innovationsförderung sowie fachlichen Verbänden auf Bundes- und regionaler Ebene.

Die Stärke des Programms FEMtech liegt u. a. auch in den handelnden Personen. Die Programm-UmsetzerInnen wurden sowohl von FTI-politischen AkteurInnen als auch von NutzerInnen des Programms FEMtech als glaubwürdig, engagiert und professionell empfunden. Der zukünftige Programmerfolg ist jedoch nicht nur von den Programm-UmsetzerInnen zu verantworten, sondern im Wesentlichen von der politischen Verantwortlichkeit für Forschung, Technologie und Innovation in Österreich und auch all jener politischen AkteurInnen, die teilweise oder indirekt zur Rahmengestaltung von Chancengleichheit beitragen. Sie entscheiden über Ernsthaftigkeit und Nachdrücklichkeit, ob Chancengleichheit von Frauen und Männern im österreichischen Innovationssystem bloß eine verbale Zuschreibung oder Selbstverständlichkeit wird. 


\section{Literatur- und Quellenverzeichnis}

Für die Evaluierung wurden sämtliche Dokumente, Broschüren und Studien, die über die Website www.femtech.at verfügbar sind, einbezogen. Weiters interne Leistungsberichte und Dokumente, die in ihrer Vielzahl hier nicht aufgelistet werden.

\section{Literatur}

Bergmann, Nadja / Pimminger Irene (2004): Praxishandbuch Gendermainstreaming.

URL: http://www.lrsocialresearch.at/files/GeM_Praxishandbuch.pdf [letzter Zugriff am 29.03.2011]

BLK, Bund-Länder-Kommission für Bildungsplanung und Forschungsförderung (2007): HWP Abschlussbericht 2007,(URL: http://www.blkinfo.de/fileadmin/Papers/hwp-abschlussbericht-2007.pdf).

European Commission (2003a): She Figures 2003 : Women and Science. Statistics and Indicators. (Science and Society). Brüssel.

European Commission (2003b): Women in Industrial Research. Analysis of Statistical Data and Good Practices of Companies. Brüssel. (URL: http://europa.eu.int/comm/research/science-society/women/wir/pdf/wirulb_en.pdf).

European Commission, Hg. (2003c): Women in Industrial Research: A Wake up Call for European Industry. Brüssel: Office for Official Publications of the European Communities

European Commission (2004): Women in Industrial Research. Speeding up changes in Europe. Proceedings of the Conference "Women in Industrial Research - Speeding up Changes in Europe!", Berlin (Germany), 10 and 11 October 2003, Luxembourg (URL: http://ec.europa.eu/research/sciencesociety/women/wir/pdf/wir_proceedings_en.pdf).

European Commission (2008): Benchmarking Policy Measures for Gender Equality in Science, Luxembourg (URL: http://ec.europa.eu/research/sciencesociety/document_library/pdf_06/benchmarking-policy-measures_en.pdf).

European Commission (2009): She Figures 2009, Luxembourg.

FEMtech (o.J.a): Wissen / Daten / Außeruniversitäre Forschung / URL: http://www.femtech.at/index.php?id=286 [letzter Zugriff am 11.5.2010].

FEMtech (o.J.b): Aktivitäten URL: http://www.femtech.at/index.php?id=29 [letzter Zugriff: 11.04.2011]

FEMtech (o.J.c): Wissen URL: http://www.femtech.at/index.php?id=47 [letzter Zugriff: 11.04 .2011$]$

FEMtech (2007): Programmdokument und Sonderrichtlinie - Programm FEMtech fFORTE Laufzeit 2007-2010; Wien 
GenderKompetenzzentrum (о.J.): Chancengleichheit URL: http://www.genderkompetenz.info/genderkompetenz/gender/gendermains trea-

ming/Strategie/Gleichstellungspolitik/chancengleichheit/index.html?sear chterm=glossa [letzter Zugriff am 29.03.2011]

Löther, Andrea / Brigitte Mühlenbruch (2004): Gleichstellungspolitik in den Hochschulsonderprogrammen und im Hochschul- und Wissenschaftsprogramm. In: Andrea Löther (Hg.), Erfolg und Wirksamkeit von Gleichstellungsmaßnahmen an Hochschulen. Bielefeld: Kleine, S. 22- 37.

Rat für Forschung und Technologieentwicklung (2002): Nationaler Forschungsund Innovationsplan, Wien

Rat für Forschung und Technologieentwicklung (2005): Strategie 2010, Perspektiven für Forschung, Technologie und Innovation in Österreich, Wien

Rees, Teresa (2002): National policies on women and science in Europe. (The Helsinki Group on Women and Science). Luxemburg: European Commission. (URL: http://cordis.europa.eu/improving/women/policies.htm).

Spreyermann, Christine / Christine Rothmayr (2008): Evaluation Bundesprogramm Chancengleichheit von Frau und Mann an Universitäten 20002007. Bericht zu den Leistungen und Wirkungen des Programms 2000 bis 2007, Bern (URL: http://www.crus.ch/dms.php?id=7861).

Statistik Austria (2010): Statistisches Jahrbuch 2011. Wien. 


\section{Abbildungs- und Tabellenverzeichnis}

Abbildung 1: Partnerorganisationen der ARGE „3C“ 1

Abbildung 2: Design Programmevaluation FEMtech ....................................................................

Abbildung 3: Maßnahmenmix FEMtech Programmevaluation ......................................................6

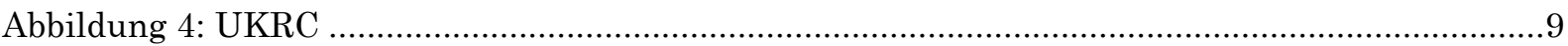

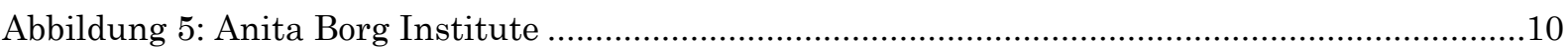

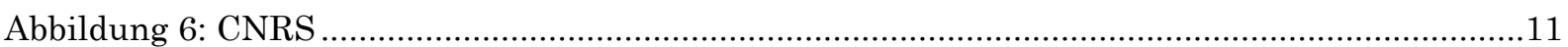

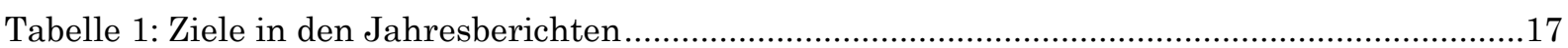

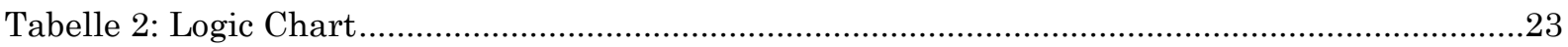

Tabelle 3: Die wesentlichen Eckpunkte von FEMtech Karriere:....................................................29

Abbildung 7: Anzahl der geförderten FEMtech Karriere-Projekte im Zeitverlauf ..........................29

Tabelle 4: Förderquote FEMtech Karriere-Projekte..............................................................................30

Tabelle 5: Gesamtkosten und Fördervolumina der genehmigten FEMtech Karriere-Projekte .......30

Abbildung 8: Geförderte FEMtech Karriere-Projekte nach Organisationstyp .................................31

Abbildung 9: Geförderte FEMtech Karriere-Projekte nach Bundesland ...........................................31

Abbildung 10: Inhaltliche Schwerpunkte FEMtech Karriere-Projekte............................................32

Tabelle 6: Akquisitionsaufwand und -erfolg FEMtech Karriere 2008-2010 ....................................33

Tabelle 7: Die wesentlichen Eckpunkte von FEMtech Karrierewege …………................................34

Abbildung 11: Übersicht genehmigter und nicht genehmigter FEMtech Karrierewege-Projekte..34

Tabelle 8: Förderquote FEMtech Karrierewege-Projekte

Tabelle 9: Gesamtkosten und Fördervolumina der genehmigten FEMtech Karrierewege-Projekte35

Abbildung 12: Übersicht Organisationstyp Konsortialpartner bei genehmigten FEMtech

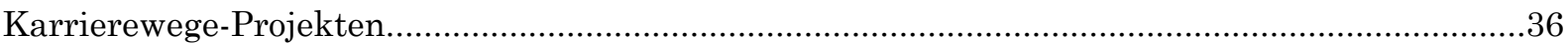

Abbildung 13: Bundesländerverteilung aller beteiligten FEMtech Karrierewege-Projektpartner.36

Abbildung 14: Inhalte der FEMtech Karrierewege-Projekte

Tabelle 10: Auswahl FWF-Personalkostensätze mit Relevanz zu Beschäftigungsverhältnissen in FEMtech Karrierewegeprojekten.

Tabelle 11: Die wesentlichen Eckpunkte von FEMtech FTI-Projekten ...........................................39

Abbildung 15: Übersicht genehmigter und abgelehnter FEMtech FTI-Projekte .............................39

Tabelle 12: Förderquote FEMtech FTI-Projekte ....................................................................................

Tabelle 13: Gesamtkosten und Fördervolumina der genehmigten FEMtech FTI-Projekte .............40

Abbildung 16: Projektträger nach Organisationstypen bei genehmigten FEMtech FTI-Projekten40

Abbildung 17: Projektpartner nach Organisationstypen bei genehmigten FEMtech FTI-Projekten41 
Abbildung 18: Bundesländer der geförderten FEMtech FTI-Träger.

Abbildung 19: Vergleich Organisationsstruktur aller FFG-Programme mit FEMtech Förderlinien43

Abbildung 20: Vergleich Bundesländerbeteiligung aller FFG-Programme mit FEMtech Förderlinien.

Abbildung 21: Vergleich Unternehmensstruktur aller FFG-Programme und FEMtech

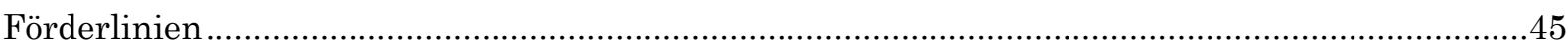

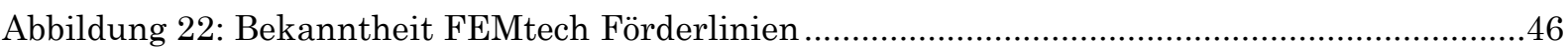

Abbildung 23: Zufriedenheit mit FEMtech Fördermanagement ....................................................

Tabelle 14: Ansatzpunkte zur Förderung von Chancengleichheit (zusammenfassende Übersicht) 50

Abbildung 24: Bekanntheit und Nutzung FEMtech Aktivitäten ..................................................54

Tabelle 15: Nutzung FEMtech Website (Anzahl unterschiedlicher BesucherInnen) ......................56

Tabelle 16: Teilnahmen bzw. Teilnehmende Netzwerktreffen ....................................................58

Tabelle 17: Teilnehmende Netzwerktreffen - nach Organisationszugehörigkeit ............................60

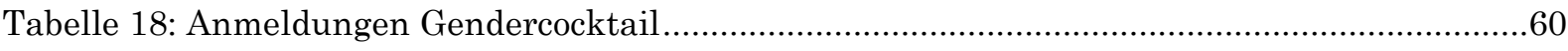

Tabelle 19: Teilnehmende Gendercocktail - nach Organisationszugehörigkeit..............................61

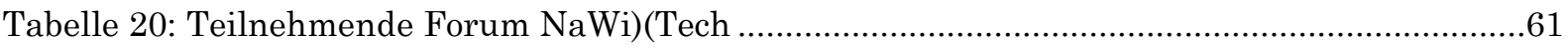

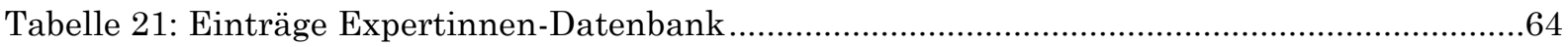

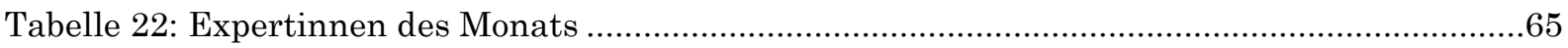

Abbildung 25: Zufriedenheit mit FEMtech Aktivitäten - nach FTI-Organisationstyp ...................66

Tabelle 23: Themen der FEMtech Publikationen ..............................................................................73

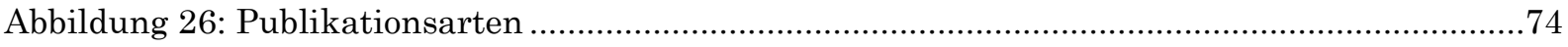

Abbildung 27: Bekanntheit und Nutzung FEMtech Wissen...........................................................76

Abbildung 28: Zufriedenheit mit FEMtech Wissen - nach FTI-Organisationstyp ..........................77

Tabelle 24: Anzahl Pressemitteilungen und Anzeigenschaltungen ...............................................78

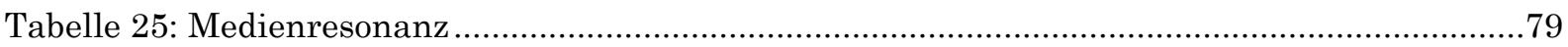

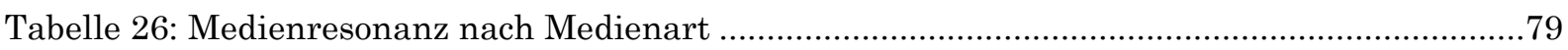

Tabelle 27: Medienresonanz nach Nennung FEMtech Angebote ......................................................80

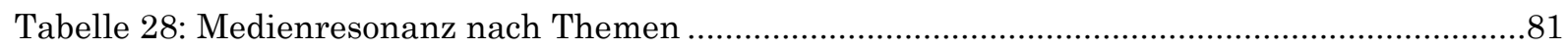

Tabelle 29: Medienresonanz nach Nennung zentraler AkteurInnen .............................................82

Abbildung 29: Einschätzung der Wichtigkeit von Humanressourcen-Themen durch FTI-politische

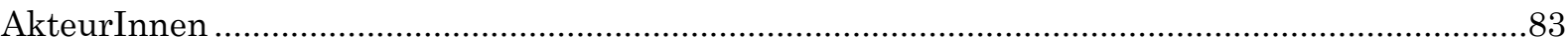

Abbildung 30: Einschätzung der Wirksamkeit von Strategien zur Herstellung von

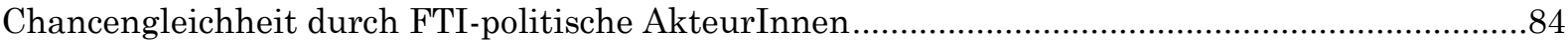

Abbildung 31: Einschätzung der Wirksamkeit von Strategien zur Herstellung von

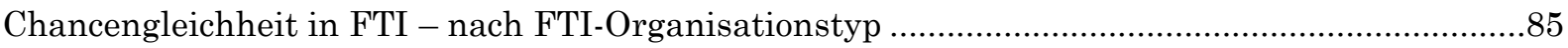

Abbildung 32: Einschätzung der Wichtigkeit von Humanressourcen-Themen - nach FTI-

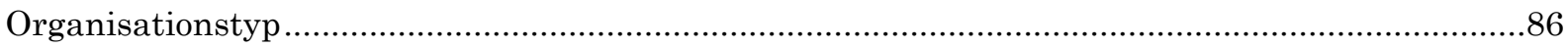




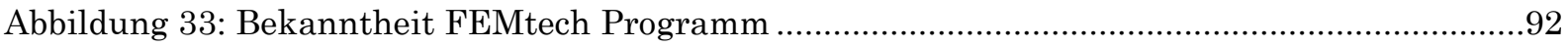

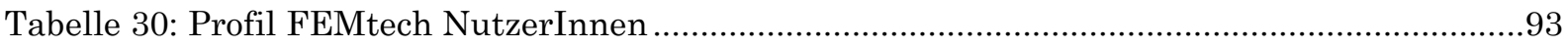

Abbildung 34: FEMtech NutzerInnen - nach Befragtengruppe....................................................95

Abbildung 35: FEMtech NutzerInnen in FTI-Unternehmen/-Organisationen - nach FTI-

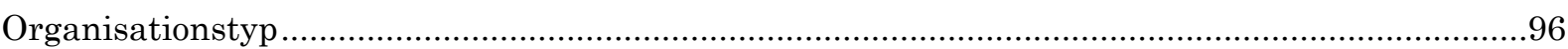

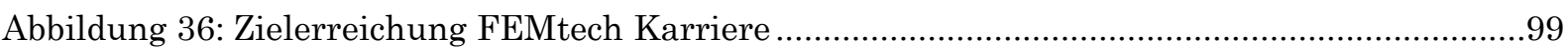

Abbildung 37: Zielerreichung FEMtech Karrierewege....................................................................100

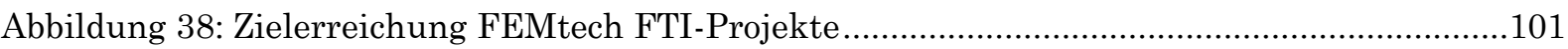

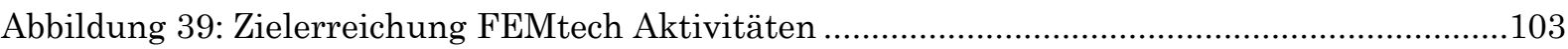

Abbildung 40: Zielerreichung FEMtech Wissensangebote............................................................104

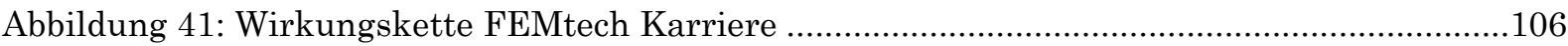

Abbildung 42: Wirkungskette FEMtech Karrierewege ……........................................................107

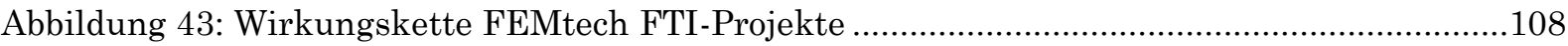

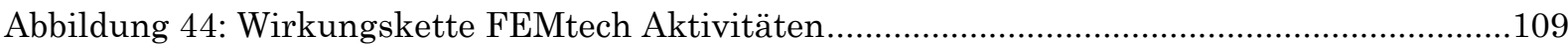

Abbildung 45: Wirkungskette FEMtech Wissen ..........................................................................110

Abbildung 46: FEMtech Programmhistorie ……….................................................................113

Abbildung 47: Mainstreamingprozess - Einordnung Programm FEMtech ...................................119

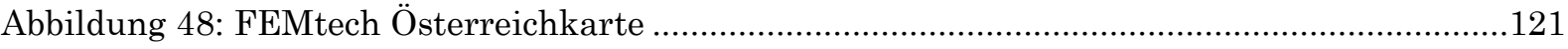




\section{A Anhang: Evaluationsmethoden}

\begin{tabular}{|c|c|}
\hline \multicolumn{2}{|c|}{$\begin{array}{l}\text { Onlinebefragung } \\
\text { (FFG Datensatz) }\end{array}$} \\
\hline Ziel & $\begin{array}{l}\text { ProjektleiterInnen von technologie- und forschungsintensi- } \\
\text { ven Organisationen, die seit } 2004 \text { Forschungsförderung bei } \\
\text { der FFG beantragt haben und deren Projekte im Falle einer } \\
\text { Bewilligung spätestens im März } 2010 \text { gestartet sind. Ausge- } \\
\text { nommen sind Organisationen, deren Genehmi- } \\
\text { gung/Ablehnung erst kürzlich erfolgte bzw. noch aussteht. } \\
\text { Ausschluss: FEMtech NutzerInnen oder Ansprechpersonen } \\
\text { aus Organisationen bzw. Einrichtungen, die eine FEMtech } \\
\text { Förderung erhalten haben, sind in dieser Gruppe nicht ent- } \\
\text { halten. }\end{array}$ \\
\hline $\begin{array}{l}\text { Grundgesamt- } \\
\text { heit }\end{array}$ & $\begin{array}{l}\mathrm{N}=9.940 \text { antragstellende Personen } \\
\text { (ProjektleiterInnen oder technische Ansprechpersonen aus } \\
\text { forschungs- und technologieintensiven Organisationen, über } \\
\text { deren Antrag auf FFG-Forschungsförderung entschieden } \\
\text { wurde. }\end{array}$ \\
\hline Rücklauf & $\begin{array}{l}\mathrm{n}=1.583 \text {, Rücklaufquote: } 17 \% \text { (als repräsentativ zu be- } \\
\text { zeichnen) }\end{array}$ \\
\hline $\begin{array}{l}\text { Erhebungsein- } \\
\text { heit }\end{array}$ & $\begin{array}{l}\text { ProjektleiterInnen von einreichenden Organisationen } d . h \text {. } \\
\text { pro Organisation können mehrere Personen zur Befragung } \\
\text { eingeladen werden, wenn das Unternehmen mehrere FFG- } \\
\text { Förderungen seit } 2004 \text { hat. }\end{array}$ \\
\hline $\begin{array}{l}\text { Auswahlver- } \\
\text { fahren }\end{array}$ & $\begin{array}{l}\text { Vollerhebung } \\
\text { Die Einladung zur Befragung erfolgte nach den o. g. Krite- } \\
\text { rien durch die FFG. }\end{array}$ \\
\hline $\begin{array}{l}\text { Erhebungs- } \\
\text { zeitraum }\end{array}$ & $\begin{array}{l}\text { 1.10. bis } 22.10 .2010 \\
\text { Reminder E-Mail am 15.10.2010 }\end{array}$ \\
\hline
\end{tabular}




\begin{tabular}{|c|c|}
\hline \multicolumn{2}{|c|}{ Onlinebefragung | FEMtech AdressatInnen und -Fördernehmende } \\
\hline Zielgruppe & $\begin{array}{l}\text { FEMtech AdressatInnen, die regelmäßig aktiv durch das } \\
\text { Programm informiert werden bzw. Angebote des Programms } \\
\text { aus den drei Bereichen Förderung, Aktivitäten und Wissen } \\
\text { bereits genutzt haben. }\end{array}$ \\
\hline $\begin{array}{l}\text { Grundgesamt- } \\
\text { heit }\end{array}$ & $\begin{array}{l}\text { FEMtech Fördernehmende }(\mathbf{n}=\mathbf{1 1 9}) \\
\text { Geförderte Institutionen der drei Förderlinien Karriere, } \\
\text { Karrierewege und FTI-Projekte (vertreten durch die jeweili- } \\
\text { ge Projektleitung) } \\
\text { FEMtech AdressatInnen }(\mathbf{n}=\mathbf{3 . 1 2 0}) \\
\text { AdressatInnen des FEMtech Adressverteilers des Kompe- } \\
\text { tenzzentrums (darin enthalten: EmpfängerInnen FEMtech } \\
\text { Newsletter, EmpfängerInnen sonstiger aktiv versendeter } \\
\text { Publikationen und Produkte sowie Teilnehmende an FEM- } \\
\text { tech Veranstaltungen). }\end{array}$ \\
\hline Rücl & $\begin{array}{l}\text { FEMtech Fördernehmende } \\
\mathrm{n}=37, \text { Rücklaufquote: } 31 \% \\
\text { FEMtech AdressatInnen } \\
\mathrm{n}=352, \text { Rücklaufquote: } 11 \%\end{array}$ \\
\hline $\begin{array}{l}\text { Erhebungsein- } \\
\text { heit }\end{array}$ & $\begin{array}{l}\text { Person (d. h. pro Organisation können mehrere Personen zur } \\
\text { Befragung eingeladen werden) }\end{array}$ \\
\hline $\begin{array}{l}\text { Auswahlverfah- } \\
\text { ren }\end{array}$ & $\begin{array}{l}\text { Vollerhebung } \\
\text { Die Einladung zur Befragung der FEMtech Fördernehmen- } \\
\text { den erfolgte nach den o. g. Kriterien durch die FFG. } \\
\text { Die Einladung der FEMtech AdressatInnen erfolgte durch } \\
\text { Bohmann Druck und Verlag. }\end{array}$ \\
\hline $\begin{array}{l}\text { Erhebungs- } \\
\text { zeitraum }\end{array}$ & $\begin{array}{l}\text { 1.10. bis } 22.10 .2010 \\
\text { Reminder E-Mail am 15.10.2010 (bei FEMtech Förderneh- } \\
\text { menden zusätzlich am 22.10.2010) }\end{array}$ \\
\hline
\end{tabular}




\begin{tabular}{|c|c|}
\hline \multicolumn{2}{|c|}{ Interviews | Oberes Management (oMan) } \\
\hline Zielgruppe & $\begin{array}{l}\text { - F\&E-LeiterInnen, Personalverantwortliche, Geschäftsfüh- } \\
\text { rerInnen (insbes. Bei KU) }\end{array}$ \\
\hline Methode & $\begin{array}{l}\text { - Qualitative Leitfadeninterviews } \\
\text { - Persönlich, z. T. telefonisch (pragmatische Entscheidung, } \\
\text { da österreichweit = regionale Streuung) } \\
\text { - Dauer ca. } 30 \text { Minuten } \\
\text { - Anonyme Auswertung } \\
\text { - N = 12, Mix aus Unternehmen unterschiedlicher Branchen } \\
\text { sowie außeruniversitären Forschungseinrichtungen }\end{array}$ \\
\hline Inhalte & $\begin{array}{l}\text { Erwartungen, Bedürfnisse, Problemanalyse bezüglich Gleich- } \\
\text { stellung in FuE, Einschätzungen zu Notwendigkeit und Nut- } \\
\text { zen FEMtech. } \\
\text { Bekanntheit, Nutzung von FEMtech Leistungen. }\end{array}$ \\
\hline $\begin{array}{l}\text { Erhebungs- } \\
\text { zeitraum }\end{array}$ & September bis Oktober 2010 \\
\hline
\end{tabular}

\begin{tabular}{|c|c|}
\hline \multicolumn{2}{|c|}{ Fokusinterviews | FEMtech Fördernehmerinnen (FN) } \\
\hline Zielgruppe & $\begin{array}{l}\text { - Nutzerinnen der drei FEMtech Förderlinien und FEMtech } \\
\text { Expertin des Monats, ergänzt durch externe Fachexpertise } \\
\text { durch Gleichstellungsbeauftragte an Hochschulen }\end{array}$ \\
\hline Methode & $\begin{array}{l}\text { - Qualitative Leitfadeninterviews } \\
\text { - persönlich und telefonisch } \\
\text { - Dauer ca. } 30 \text { Minuten } \\
\text { - Anonyme Auswertung } \\
\text { - } \mathrm{N}=9\end{array}$ \\
\hline Inhalte & $\begin{array}{l}\text { Erwartungen, Bedürfnisse, Problemanalyse bezüglich Chan- } \\
\text { cengleichheit in FuE, Einschätzungen zu Notwendigkeit und } \\
\text { Nutzen FEMtech. } \\
\text { Auswirkungen von FEMtech Leistungen in persönlicher } \\
\text { Wahrnehmung. }\end{array}$ \\
\hline $\begin{array}{l}\text { Erhebungs- } \\
\text { zeitraum }\end{array}$ & Dezember 2010 bis Jänner 2011 \\
\hline
\end{tabular}




\begin{tabular}{l|l}
\multicolumn{2}{l}{ Nutzungsanalyse | FEMtech Website } \\
\hline Zielgruppe & $\begin{array}{l}\text { - Personen, die die Website besuchen / aktiv nach Informa- } \\
\text { tionen suchen }\end{array}$ \\
\hline Methode & $\begin{array}{l}\text { 1) Analyse der quantitativen Nutzung im Zeitverlauf } \\
\text { - Basis: FEMtech Homepage Statistik } \\
\text { 2) On-Site-Kurzbefragung der Website-NutzerInnen }\end{array}$ \\
& $\begin{array}{l}\text { - Einladung der Website-NutzerInnen über verschiedene } \\
\text { Zugänge (integriertes Popup-Fenster, prominent platzier- } \\
\text { ter Link) auf der FEMtech Website selbst }\end{array}$ \\
& - Direkter Link zur Befragung (Dauer max. 5 Minuten) \\
& - Befragungszeitraum: 10 Wochen
\end{tabular}

\begin{tabular}{|c|c|}
\hline \multicolumn{2}{|c|}{ Medienresonanzanalyse | FEMtech in Medien } \\
\hline Zielgruppe & - FEMtech Zielgruppen, allgemeine Öffentlichkeit \\
\hline Methode & $\begin{array}{l}\text { Qualitative Medienresonanzanalyse } \\
\text { - Basis: Durch das Kompetenzzentrum zur Verfügung ge- } \\
\text { stellte Presseaussendungen und gesammelte Medienreso- } \\
\text { nanz }\end{array}$ \\
\hline $\begin{array}{l}\text { Erhebungs- } \\
\text { einheit }\end{array}$ & $\begin{array}{l}230 \text { Clippings } \\
66 \text { Medienschaltungen } \\
63 \text { Pressemitteilungen } \\
22 \text { APA OTS-Online-Aussendungen }\end{array}$ \\
\hline Inhalte & $\begin{array}{l}\text { Formale Kriterien: Reichweite (sofern in der Resonanz er- } \\
\text { fasst), Medienart etc. } \\
\text { Inhaltliche Kriterien: Zitierte AkteurInnen, Meinungstenor } \\
\text { der Berichterstattung, vermittelte Botschaften und Argu- } \\
\text { mente etc. }\end{array}$ \\
\hline $\begin{array}{l}\text { Erhebungs- } \\
\text { zeitraum }\end{array}$ & August 2005 bis Juni 2010 \\
\hline
\end{tabular}




\begin{tabular}{|c|c|}
\hline \multicolumn{2}{|c|}{ Produktanalyse | FEMtech Outputs } \\
\hline Methode & $\begin{array}{l}\text { Produktanalyse (Kriteriengeleitete Begutachtung der Pro- } \\
\text { dukte) } \\
\text { - Basis: Output des Programms zur Nutzung für die Ziel- } \\
\text { gruppe (= Sämtliche Produkte aus den Bereichen Aktivi- } \\
\text { täten, Förderungen und Wissen) }\end{array}$ \\
\hline Inhalte & $\begin{array}{l}\text { Analyse der Produkte im Hinblick auf folgende Kriterien: } \\
\text { - Inhalte/Themen } \\
\text { - Nutzung (erreichte Zielgruppen) } \\
\text { - Zufriedenheit der Zielgruppen } \\
\text { - Gestaltungshinweise der Zielgruppen } \\
\text { Vergleich mit Outputs ähnlicher Einrichtungen internatio- } \\
\text { nal }\end{array}$ \\
\hline
\end{tabular}

\begin{tabular}{l|l}
\multicolumn{2}{l}{ Interviews | FTI-politische Akteure (FTI-pA) } \\
\hline Zielgruppe & $\begin{array}{l}\text { - Opinion Leader im Bereich FTI-Politik - Auswahl in Ab- } \\
\text { stimmung mit der Auftraggeberin }\end{array}$ \\
\hline Methode & $\begin{array}{l}\text { Qualitative Leitfadeninterviews } \\
\text { - Persönlich } \\
\text { - Dauer ca. 45-60 Minuten } \\
\text { - Anonyme Auswertung } \\
\text { - N }=6\end{array}$ \\
\hline Inhalte & $\begin{array}{l}\text { Problemanalyse bezüglich Gleichstellungsmaßnahmen in der } \\
\text { FTI-Politik, Hebel für effektive Unterstützung von Chancen- } \\
\text { gleichheit in der FTI-Politik, Nutzen von FEMtech für die }\end{array}$ \\
& FTI-Politik. \\
\hline $\begin{array}{l}\text { Erhebungs- } \\
\text { zeitraum }\end{array}$ & August bis Oktober 2010
\end{tabular}

\begin{tabular}{l|l}
\multicolumn{2}{l}{ Programmgespräch | FEMtech UmsetzerInnen } \\
\hline Zielgruppe & $\begin{array}{l}\text { FEMtech Programm-UmsetzerInnen = BMVIT, FFG, Kompe- } \\
\text { tenzzentrum }\end{array}$ \\
\hline Methode & $\begin{array}{l}\text { Gespräche im Interviewstil } \\
\text { - Persönlich } \\
\text { - Dauer ca. 1,5-2,5 Stunden } \\
\text { - N = 6 }\end{array}$ \\
\hline Inhalte & $\begin{array}{l}\text { Ziele, Programmkonzeption, Programmentwicklung, Organi- } \\
\text { sation des Kompetenzzentrums, Einbindung in die FTI- }\end{array}$ \\
& $\begin{array}{l}\text { Förderungsaktivitäten, Einschätzungen zu bisherigen Erfol- } \\
\text { gen des Programms FEMtech. }\end{array}$ \\
\hline $\begin{array}{l}\text { Erhebungs- } \\
\text { zeitraum }\end{array}$ & $\begin{array}{l}\text { August bis Oktober 2010 } \\
\end{array}$
\end{tabular}




\begin{tabular}{l|l}
\multicolumn{2}{l}{ Self-Assessment | FEMtech UmsetzerInnen } \\
\hline Zielgruppe & $\begin{array}{l}\text { FEMtech Programm-UmsetzerInnen = BMVIT, FFG, Kompe- } \\
\text { tenzzentrum }\end{array}$ \\
\hline Methode & $\begin{array}{l}\text { Self-Assessment: } \\
\text { - Schriftlicher Selbstausfüller } \\
\text { - Dauer ca. 1,5 Stunden } \\
\text { - Anonyme Auswertung }\end{array}$ \\
& - N=12 \\
\hline Inhalte & $\begin{array}{l}\text { Selbsteinschätzungen zu Stärken und Schwächen des Pro- } \\
\text { gramms, Organisation der Programmumsetzung, Einschät- } \\
\end{array}$ \\
& $\begin{array}{l}\text { zungen zur Zielgruppenerreichung, Einschätzungen zu Leis- } \\
\text { tungsbereichen und Produkten von FEMtech. }\end{array}$ \\
\hline $\begin{array}{l}\text { Erhebungs- } \\
\text { zeitraum }\end{array}$ & Oktober bis Anfang November 2010 \\
\end{tabular}

Fokusinterviews | ehemalige Programmleiterinnen (PG)

\begin{tabular}{l|l}
\hline Zielgruppe & Ehemalige Programmleiterinnen \\
\hline Methode & $\begin{array}{l}\text { Offene Interviews mit ehemaligen FEMtech } \\
\text { Programmleiterinnen ergänzt mit „FEMtech Historienge- } \\
\text { spräch“ mit aktiven Leiterinnen im BMVIT } \\
\text { - Dauer ca. 2 Stunden } \\
\text { - Anonyme Auswertung } \\
\text { - N =6 }\end{array}$ \\
\hline Inhalte & $\begin{array}{l}\text { Geschichtliche Dimension: FEMtech Phasen in der Perfor- } \\
\text { mance, Veränderungen des Programms, Einschätzung zu } \\
\end{array}$ \\
& $\begin{array}{l}\text { Produkten, Ziel- und Zielgruppenerreichung, Einbindung in } \\
\text { die FTI-Förderungspolitik. }\end{array}$ \\
\hline Erhebungs- & November 2010 bis Jänner 2011 \\
Zeitraum &
\end{tabular}




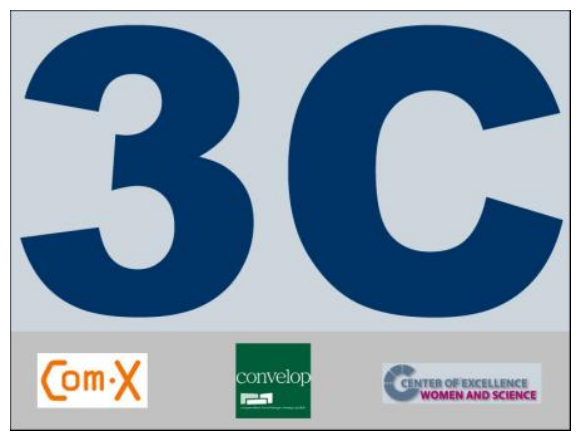

convelop

cooperative knowledge design gmbh

A-8010 Graz Bürgergasse 8-10/

Telefon: $\quad+43(0) 316720813$

Fax: $\quad+43(0) 316720$ 813-20

www.convelop.at office@convelop.at
Com.X Institut für Kommunikationsanalyse und Evaluation

D-44789 Bochum Ehrenfeldstr. 34 Tel.: $\quad+49(0) 2343250830$ Fax: $\quad+49(0) 2343250831$ www.comx-forschung de
GESIS Leibniz-Institut für Sozialwissenschaften / CEWS

D-68159 Mannheim Quadrat B2, 1 Tel.: $\quad+49(0) 2282281522$ Fax: $\quad+49(0) 2282281550$ www.gesis.org www.cews.org 